\title{
Acquired alteration in platelets
}

Citation for published version (APA):

Baaten, C. C. F. M. J. B. (2018). Acquired alteration in platelets: insight into impairment and recovery of platelet function. [Doctoral Thesis, Maastricht University]. Maastricht University. https://doi.org/10.26481/dis.20180315cb

Document status and date:

Published: 01/01/2018

DOI:

$10.26481 /$ dis.20180315cb

Document Version:

Publisher's PDF, also known as Version of record

\section{Please check the document version of this publication:}

- A submitted manuscript is the version of the article upon submission and before peer-review. There can be important differences between the submitted version and the official published version of record.

People interested in the research are advised to contact the author for the final version of the publication, or visit the DOI to the publisher's website.

- The final author version and the galley proof are versions of the publication after peer review.

- The final published version features the final layout of the paper including the volume, issue and page numbers.

Link to publication

\footnotetext{
General rights rights.

- You may freely distribute the URL identifying the publication in the public portal. please follow below link for the End User Agreement:

www.umlib.nl/taverne-license

Take down policy

If you believe that this document breaches copyright please contact us at:

repository@maastrichtuniversity.nl

providing details and we will investigate your claim.
}

Copyright and moral rights for the publications made accessible in the public portal are retained by the authors and/or other copyright owners and it is a condition of accessing publications that users recognise and abide by the legal requirements associated with these

- Users may download and print one copy of any publication from the public portal for the purpose of private study or research.

- You may not further distribute the material or use it for any profit-making activity or commercial gain

If the publication is distributed under the terms of Article $25 \mathrm{fa}$ of the Dutch Copyright Act, indicated by the "Taverne" license above, 


\section{Acquired alterations in platelets: \\ Insight into impairment and recovery of platelet function}

Constance C.F.M.J. Baaten 
Acquired alterations in platelets:

Insight into impairment and recovery of platelet function

Thesis Maastricht University

ISBN 978-94-028-0888-9

Production: Ipskamp Printing

(C) Constance Baaten, Maastricht 2018

Cover design by Evelien Jagtman (www.evelienjagtman.com) 


\section{Acquired alterations in platelets: \\ Insight into impairment and recovery in platelet function}

\section{Proefschrift}

Ter verkrijging van de graad van doctor aan de Universiteit Maastricht, op gezag van de Rector Magnificus, Prof. Dr. Rianne M. Letschert, volgens het besluit van het College van Decanen, in het openbaar te verdedigen op

donderdag 15 maart 2018 om 10.00 uur

door

Constance Catharina Francoise Maria Josepha Baaten

Geboren te Maasbracht op 5 december 1988 


\section{Promotor}

Prof. Dr. J.W.M. Heemskerk

\section{Copromotor}

Dr. P.E.J. van der Meijden

\section{Beoordelingscommissie}

Prof. Dr. H.C. Schouten (voorzitter)

Prof. Dr. T.M. Hackeng

Prof. Dr. H. Philippou (University of Leeds, United Kingdom)

Dr. H.M.H. Spronk

Prof. Dr. J.J. Zwaginga (Leids Universitair Medisch Centrum)

Financial support for publication of this thesis by Stichting Hartsvrienden RESCAR is gratefully acknowledged.

Financial support by the Dutch Heart Foundation for publication of this thesis is gratefully acknowledged. 


\section{Contents}

$\begin{array}{llr}\text { Chapter } 1 & \text { General Introduction } & 7\end{array}$

Chapter $2 \quad$ Platelet populations and priming in hematological diseases 19

Chapter 3 Gradual increase in thrombogenicity of juvenile platelets formed upon offset of prasugrel medication

Chapter $4 \quad$ Platelet control of fibrin distribution and microelasticity in thrombus formation under flow

Chapter 5 Platelet heterogeneity in glycoprotein shedding: consequence for procoagulant responsiveness

Chapter $6 \quad$ Impaired mitochondrial activity explains platelet dysfunction in thrombocytopenic cancer patients undergoing chemotherapy

Chapter 7 Additive roles of platelets and fibrinogen in whole blood fibrin clot formation upon dilution as assessed by thromboelastometry

Chapter 8 A synthesis approach of mouse studies to identify new genes and proteins in arterial thrombosis and bleeding

Chapter 9 General Discussion

Chapter 10 Summary

Samenvatting

Valorization

Curriculum Vitae

Publications

Dankwoord 



\section{Chapter 1}

General Introduction 
Platelets play pivotal roles in thrombosis and hemostasis. Upon vascular injury, they acutely respond by forming a plug or aggregate, and thereby prevent further blood loss. Following activation of the coagulation cascade, a fibrin mesh is formed, which stabilizes the platelet plug. This process of clot formation is referred to as hemostasis. ${ }^{1}$ When platelet activation or coagulation is impaired due to either genetic or environmental factors, hemostatic clot formation is hampered, which leads to an increased risk of hemorrhage. On the contrary, enhanced platelet activation or coagulation relates to a higher risk of thrombosis, which is the pathological process where aberrant clot formation results in occlusion of a blood vessel. While platelets are considered to have a predominant role in arterial thrombosis, venous thrombosis is more dependent on the coagulation process. ${ }^{2,3}$

In the present chapter, a general overview is given of platelet functions and of thrombus formation, as far as relevant for the present thesis. Furthermore, a brief description is provided on acquired clinical conditions that are linked to either bleeding or thrombosis.

\section{Regulation of platelet functions and thrombus formation}

The formation of new platelets takes place mainly, but not exclusively, in the bone marrow. ${ }^{4,5}$ Proplatelets are shed from the precursor megakaryocytes in a process called thrombopoiesis. ${ }^{6}$ These proplatelets further divide to form the individual platelets that stay in circulation. In man, platelets circulate for 7-10 days on average, meaning that a continuous production of new platelets is needed to maintain a constant platelet count. $^{7}$

Newly formed platelets contain measurable amounts of mRNA that is inherited from the precursor megakaryocytes; hence they are named 'reticulated platelets'. ${ }^{8}$ As the platelet mRNA degrades over time, the older circulating platelets are essentially devoid of these linear ribonucleotides, although they still contain circular RNA forms. ${ }^{9}$ The presence of mRNA is often used as a feature to identify newly formed platelets. ${ }^{10}$ In the literature, it has been suggested that reticulated platelets are larger and more reactive than the older ones. ${ }^{11,12}$ However, conclusive evidence for this suggestion is still missing, ${ }^{13,14}$ partly due to the lack of methods that enable a proper detection of reticulated platelets. Characterization of these platelet types in terms of structure and function thus requires more investigation.

Circulating platelets are capable of responding quickly to vascular damage, since they are equipped with a multitude of signaling receptors on the plasma membrane.,15 Under conditions of high shear rate, such as present in the arteries and arterioles, red blood cells normally accumulate in the central part of the vessel lumen, whereby they push the platelets outwards to come in close proximity to the vascular wall. ${ }^{16,17}$ One of the first events, upon vascular damage, is the shear-dependent interaction of these platelets with von Willebrand factor, derived from endothelial cells, that is bound to the exposed collagen fibers. ${ }^{18}$ The platelet glycoprotein Ib-V-IX complexes are primarily responsible for the interaction with von Willebrand factor. It causes platelets to roll, which then triggers their firm binding to collagen itself via the receptors glycoprotein $\mathrm{VI}$ and integrin $\alpha_{2} \beta_{1}{ }^{1,19,20}$ The collagen-attached platelets become activated, primarily through 
glycoprotein $\mathrm{VI}$, and respond by granule secretion, in which they release cargo molecules from the $\alpha$ and dense granules. Depending on the strength of the stimulus, platelets will secrete more or less of this granular cargo. ${ }^{21}$ The secretory granules contain many autocrine and paracrine molecules. Large molecules, such as fibrinogen and factor $\mathrm{V}$, are packed in the $\alpha$ granules, while the dense granules are loaded among others with ADP, ATP and polyphosphates. ${ }^{22}$ Once secreted, ATP and ADP serve as autacoids to activate nearby platelets via the purinergic receptors $\mathrm{P} 2 \mathrm{X}_{1}$ (for ATP) and $\mathrm{P}_{2} \mathrm{Y}_{1}$ plus $\mathrm{P}_{2} \mathrm{Y}_{12}$ (for ADP). Along with secretion, the autacoid thromboxane $\mathrm{A}_{2}$ is produced via the cyclooxygenase/ thromboxane synthase complex, which similarly activates nearby platelets via the TP receptors. Together, these autocrine agents provide a potent amplification loop to enhance effects of initial triggers of platelet activation, e.g. provided by collagen or thrombin traces. ${ }^{23,24}$ Another important platelet response is the conformation change of integrin $\alpha_{11 b} \beta_{3}$, which is a glycoprotein complex abundantly expressed on the platelet surface. Activation of $\alpha_{11 \mathrm{~b}} \beta_{3}$ integrin enables the binding of fibrinogen, a protein that is necessary for platelet-platelet interactions and thus for stable platelet aggregation. ${ }^{25}$ The eventual size and activity of a platelet aggregate or a thrombus formed on collagen is furthermore influenced by the presence of other platelet-adhesive proteins in the vessel wall and by local shear stress. ${ }^{26}$

There is evidence indicating that platelets remain active for a certain time, also after being incorporated into a thrombus. For instance, the platelets in a thrombus formed on collagen secrete matrix metalloproteinases, enzymes which gradually degrade the underlying collagen fibers. ${ }^{27}$ In addition, these platelets can undergo proteolytic cleavage of several glycoproteins. ${ }^{28}$ Glycoprotein Ib $\alpha$ and glycoprotein $\mathrm{VI}$ can be cleaved by members of the A Disintegrin And Metalloprotease family, namely by ADAM17 and ADAM10, respectively, in a process called receptor shedding. ${ }^{29}$ Both ADAM 10 and 17 are membrane-bound proteases, which remove the entire extracellular domain of their target membrane proteins. ${ }^{30}$ Although shedding of glycoprotein Ib $\alpha$ and glycoprotein VI will theoretically impair the ability of platelets to bind von Willebrand factor and collagen, the physiological role of receptor shedding has remained elusive. So far, only the suggestion has been made that shedding is a mechanism to shut down platelet adhesion and activation. ${ }^{31}$ Another suggestion is that the shedding of especially glycoprotein Iba serves to clear platelets from the circulation in the liver. ${ }^{32}$

\section{Platelet heterogeneity within thrombi}

It has been established in earlier work from our laboratory that the platelets that are incorporated in a thrombus can undergo different types of responses, and thereby show different phenotypes. ${ }^{1}$ Accordingly, the composition of an average thrombus is quite heterogeneous, containing multiple platelet phenotypes (Figure 1). How individual platelets decide which phenotype to adapt is largely unknown. The immediate environment of a platelet is considered to be important, but also the composition of a 
platelet may contribute to the heterogeneity. ${ }^{33,34}$ It can be hypothesized that the content of cytoplasm and membranes that is inherited from a given precursor megakaryocyte predispose platelets to assume a certain phenotype.

Phenotypes that can be distinguished are adherent platelets - platelets that directly interact with the damaged vessel wall - and aggregating platelets, which in particular interact with each other. The majority of these aggregating platelets, which form the bulk of the thrombus, show high activation of integrin $\alpha_{111} \beta_{3}$, required for platelet aggregation and show significant levels of secretion. ${ }^{33,35}$ Upon strong activation with sustained, high intracellular $\mathrm{Ca}^{2+}$ levels, collagen-interacting platelets assume a different, procoagulant phenotype. ${ }^{35,36}$ The procoagulant platelets are characterized by the presence of negatively charged phosphatidylserine at the outer leaflet of the plasma membrane. ${ }^{37}$ Phosphatidylserine exposure is mediated by the ion channel and phospholipid scramblase anoctamin-6 (previously designated as TMEM16F), causing transbilayer movement of phospholipids over the membrane..$^{38,39}$ The exposed phosphatidylserine supports assembly of the coagulation complexes, tenase and prothrombinase, and thereby catalyzes the formation of thrombin. ${ }^{35,40}$

Tissue factor that comes into contact with blood upon vascular damage triggers the extrinsic pathway of coagulation, by forming a complex with coagulation factor VII(a) and factor $X .{ }^{41}$ Once initial traces of factor $\mathrm{Xa}$ and subsequently thrombin are formed, these cleave other coagulation factors, including factor $\mathrm{V}$. In the prothrombinase complexes at the surface of procoagulant platelets, the formed factor Xa assembles with factor $\mathrm{Va}$, which then strongly stimulates the conversion of prothrombin to thrombin. ${ }^{42,43}$ The local generation of thrombin enhances platelet activation, and also converts fibrinogen to fibrin. ${ }^{34}$ The so-called coated platelets form a specific subset of the procoagulant platelets. ${ }^{44}$ These coated platelets require co-activation with thrombin, and show high levels of fibrin(ogen) binding, despite integrin $\alpha_{11 b} \beta_{3}$ closure and contain high activity of the transglutaminase factor XIIla, which assists in platelet-dependent fibrin fiber formation. ${ }^{45}$ Consequently, a fibrin network is formed which extends from the thrombus. In vivo models of thrombosis have shown that the fibrin network is distributed in and around a thrombus in a non-homogeneous way. ${ }^{46,47}$ In spite of this knowledge, it is still unclear how the platelet activation state and local shear stress determine the spatial distribution of fibrin. 


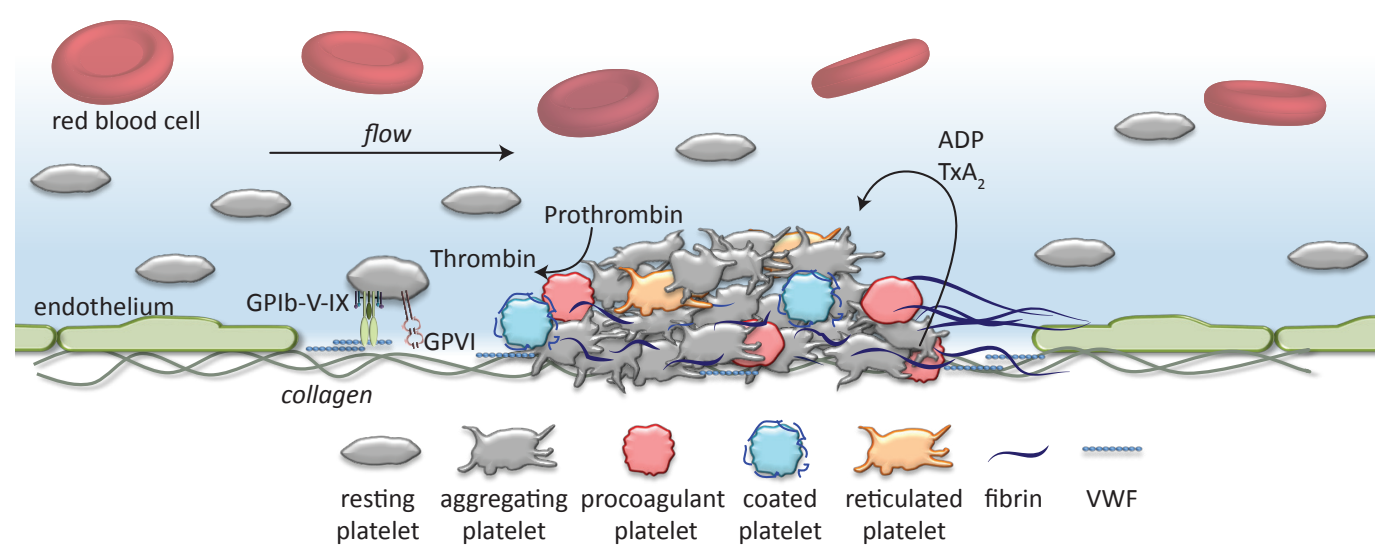

Figure 1: An overview of platelet heterogeneity in thrombus formation. Detailed explanation of the roles of different platelet agonists, receptors and platelet subtypes is given in the text.

\section{Platelets and thrombosis}

The most common cause of arterial thrombosis is rupture or erosion of an atherosclerotic plaque. ${ }^{3}$ For example, in the coronary arteries this results in partial or full occlusion, with myocardial infarction as a consequence. Patients with obstructed coronary arteries are often treated by percutaneous coronary intervention, followed by stent placement. In order to prevent recurrent ischemic events, e.g. due to in-stent thrombosis, patients receive dual antiplatelet therapy. ${ }^{48}$ The therapeutic regimen consists of a daily intake of a P2Y ${ }_{12}$ inhibitor for the period of one year, in combination with aspirin that is recommended to be taken life-long. ${ }^{49}$ The $\mathrm{P}_{2 \mathrm{Y}_{12}}$ inhibitors that are currently prescribed include the irreversible inhibitors, clopidogrel or prasugrel, or alternatively the reversible inhibitor, ticagrelor. ${ }^{23}$ The irreversible inhibitors form an active metabolite in the liver which, once released into the blood stream, specifically binds and inactivates the platelet $\mathrm{P} 2 \mathrm{Y}_{12}$ receptors. ${ }^{50}$ Aspirin, on the other hand, irreversibly abolishes the synthesis of thromboxane $A_{2}$, and thereby blocks the reinforcement of platelet response by this autacoid. ${ }^{51}$ An important drawback of the dual inhibition of both ADP/P2Y ${ }_{12}$ and thromboxane $A_{2}$ responses is a significantly increased risk of bleeding. ${ }^{23}$ Since after one year of treatment, the beneficial antithrombotic effect equalizes the negative effect of bleeding, it is advised to stop administration of the $\mathrm{P} 2 \mathrm{Y}_{12}$ inhibitors at this time point. There are indications that, during the offset period, patients are at increased risk of a recurrent ischemic event. ${ }^{52-55}$ Speculations are that this is possibly a consequence of hyperreactivity of the platelets, which are formed after treatment cessation, but this has not been studied in detail.

\section{Bleeding}

Under conditions where platelet or coagulation activation is impaired, hemostatic clot formation becomes hampered, leading to an increased risk of bleeding. As of today, the most common treatment option to prevent or treat hemorrhages is the transfusion of blood products. ${ }^{56}$ In the Netherlands, platelet transfusion is commonly performed 
with concentrates consisting of platelets pooled from five donors, suspended in plasma to which 'PAS' (platelet-additive solution) medium can be added. These pooled platelet concentrates can be stored for up to 7 days ${ }^{57}$ Although platelet transfusions temporarily increases the platelet count and hence improves hemostasis, this treatment also has potential risks, such as allergic reactions, infectious diseases and, incidentally, acute lung injury. ${ }^{56}$

Currently, a substantial part of platelet concentrates is administered to patients, who have been diagnosed with a hematological malignancy and who were therefore treated with chemotherapy. ${ }^{58}$ As a consequence of disease and foremost chemotherapeutic treatment, these patients develop severe thrombocytopenia. ${ }^{58}$ Normal platelet count ranges from $150-400 \times 10^{9} \mathrm{plts} / \mathrm{L}$, but severely thrombocytopenic patients have a platelet count lower than $50 \times 10^{9} \mathrm{plts} / \mathrm{L}$. Besides a low count, it has been reported that the platelets of these patients before onset of chemotherapy can show impairments in P-selectin expression and thromboxane $\mathrm{B}_{2}$ production, which might explain an increased bleeding risk. ${ }^{59,60}$ However, the severity and etiology of such a platelet dysfunction is unclear.

Another clinical condition in which bleeding is common is that of dilutional coagulopathy. As a consequence of major blood loss due to trauma or surgery (e.g. the cardiopulmonary bypass procedure) patients will receive fluid infusion (crystalloids and colloids) to maintain normal fluid and electrolyte homeostasis. ${ }^{61}$ As a consequence of both the blood loss and the blood dilution, coagulation factors will become diluted and thrombocytopenia will occur. In order to prevent anemia and preserve hemostasis, transfusion protocols have been developed, in which packed red blood cells, plasma and coagulation factors, and platelet concentrates are given. ${ }^{62,63}$ However, as it is desired to reduce unnecessary use of blood products, ${ }^{63}$ a better understanding of the individual and combined effects of these blood products is necessary.

\section{Experimental models for thrombosis and hemostasis}

To understand the roles of different platelet and coagulation proteins in thrombosis and bleeding, genetic mouse models have been developed, in which a specific platelet or coagulation protein is missing or defective. ${ }^{64}$ Experimentally, arterial damage can be induced in these mice to trigger thrombus formation, ${ }^{65}$ and the tail tip can be cut to monitor the bleeding phenotype. ${ }^{64}$ Taking into account specific differences between the human and mouse hemostatic systems, these genetic mouse models have provided useful insight into the process of thrombus formation, and guided in the development of new antiplatelet and anticoagulant therapies. ${ }^{66}$ As a way to reduce and refine such animal experiments, in vitro studies can be performed with isolated whole blood and microfluidic flow chambers. ${ }^{67,68}$ From a translational point of view, using such flow chambers, it is possible to study thrombus formation on multiple thrombogenic surfaces in the absence or presence of coagulation with either mouse or human blood samples. ${ }^{26,68}$ 
By systematically comparing the consequences of a genetic deficiency in various in vivo and in vitro approaches, a broader understanding of the roles of the individual genes in thrombosis and hemostasis can be gained.

\section{Aims and outline of this thesis}

The chapters of this thesis have as a common aim to better understand the consequences of an acquired impairment or recovery of platelet functions. In Chapter 1 , a general introduction is presented in order to provide relevant background information on platelet physiology and mechanisms of platelet activation during thrombus formation. Chapter 2 provides a new concept regarding the formation of different platelet populations in a physiological environment, and extends this to altered platelet phenotypes in a pathological environment. Chapter 3 describes how newly formed, juvenile platelets contribute to a recovery of function in patients, who stopped intake of the $\mathrm{P}_{2} \mathrm{Y}_{12}$ receptor inhibitor prasugrel at one year after myocardial infarction. This chapter furthermore presents a novel methodology to identify the juvenile platelets, such in comparison to established methods. Chapter 4 focuses on the dynamic processes of platelet adhesion, aggregation and fibrin clot formation under flow conditions. It is examined how immobilized collagen and tissue factor steer the formation of different types of thrombi with a specific micro-elastic structure. Thrombus phenotype and architecture are also characterized in Chapter 5. Here, a new feature of the population of procoagulant platelets is examined, namely the loss of glycoprotein Ib $\alpha$ and glycoprotein VI. It is studied how extracellular shedding of these receptors, mediated by the proteases ADAM10 and 17, affects the platelet coagulant activity. This chapter also identifies the main signaling pathways, which regulate ADAM activity in activated platelets. The central theme of Chapter 6 is identification and characterization of platelet dysfunction, which occurs in leukemia, multiple myeloma and lymphoma patients, who are treated with chemotherapy and develop thrombocytopenia as a consequence. This work focusses on the role of platelet mitochondria in relation to the dysfunction. How platelet transfusion alters the overall platelet responsiveness in these patients is studied as well. Chapter 7 examines the means for recovery of coagulant activity in patients with dilutional coagulopathy. A central question is how the supplementation of platelets and fibrinogen can alter elastic clot formation and, hence, improve the acquired hemostatic dysfunction. Chapter 8 presents a comprehensive literature synthesis, aiming to develop a method to systematically compare the consequences of genetic deficiencies in mice for arterial thrombosis and hemostasis. A scoring system is proposed allowing the calibration and comparison of multiple heterogeneous studies using different in vivo and in vitro models of arterial thrombus formation. These scores are used to construct a network of proteins in thrombosis and hemostasis, allowing the identification of new genes involved in these processes. In the last Chapter 9, the principal findings of this thesis are critically discussed in the light of the current literature. 


\section{References}

1. Versteeg HH, Heemskerk JW, Levi M, Reitsma PH. New fundamentals in hemostasis. Physiol Rev. 2013;93(1):327-358.

2. Tan KT, Lip GY. Red vs white thrombi: treating the right clot is crucial. Arch Intern Med. 2003;163(20):25342535.

3. Mackman N. Triggers, targets and treatments for thrombosis. Nature. 2008;451(7181):914-918.

4. Stegner $D$, van Eeuwijk JMM, Angay $\mathrm{O}$, et al. Thrombopoiesis is spatially regulated by the bone marrow vasculature. Nat Commun. 2017;8(1):127.

5. Lefrançais E, Ortiz-Muñoz G, Caudrillier A, et al. The lung is a site of platelet biogenesis and a reservoir for haematopoietic progenitors. Nature. 2017;544(7648):105-109.

6. Thon JN, Italiano JEJ. Does size matter in platelet production? Blood. 2012;120(8):1552-1561.

7. Lebois M, Josefsson EC. Regulation of platelet lifespan by apoptosis. Platelets. 2016;27(6):497-504.

8. Schubert S, Weyrich AS, Rowley JW. A tour through the transcriptional landscape of platelets. Blood. 2014;124(4):493-502.

9. Alhasan AA, Izuogu OG, Al-Balool HH, et al. Circular RNA enrichment in platelets is a signature of transcriptome degradation. Blood. 2016;127(9):e1-e11.

10. Harrison P, Goodall AH. "Message in the platelet"-- more than just vestigial mRNA! Platelets. 2008;19(6):395-404.

11. Rinder HM, Tracey JB, Recht $M$, et al. Differences in platelet alpha-granule release between normals and immune thrombocytopenic patients and between young and old platelets. Thromb Haemost. 1998;80(3):457-462.

12. McBane RDn, Gonzalez C, Hodge DO, Wysokinski WE. Propensity for young reticulated platelet recruitment into arterial thrombi. J Thromb Thrombolysis. 2014;37(2):148-154.

13. Robinson MS, Mackie IJ, Khair K, et al. Flow cytometric analysis of reticulated platelets: evidence for a large proportion of non-specific labelling of dense granules by fluorescent dyes. Br J Haematol. 1998;100(2):351357.

14. Hoffmann JJ, van den Broek NM, Curvers J. Reference intervals of reticulated platelets and other platelet parameters and their associations. Arch Pathol Lab Med. 2013;137(11):1635-1640.

15. Swieringa F, Kuijpers MJ, Heemskerk JW, van der Meijden PE. Targeting platelet receptor function in thrombus formation: the risk of bleeding. Blood Rev. 2014;28(1):9-21.

16. Aarts PA, van den Broek SA, Prins GW, et al. Blood platelets are concentrated near the wall and red blood cells, in the center in flowing blood. Arteriosclerosis. 1988;8(6):819-824.

17. Zwaginga JJ, Nash G, King MR, et al. Flow-based assays for global assessment of hemostasis. Part 1: Biorheologic considerations. J Thromb Haemost. 2006;4(11):2486-2487.

18. Ruggeri ZM, Mendolicchio GL. Interaction of von Willebrand factor with platelets and the vessel wall. Hamostaseologie. 2015;35(3):211-224.

19. Siljander PR, Munnix IC, Smethurst PA, et al. Platelet receptor interplay regulates collagen-induced thrombus formation in flowing human blood. Blood. 2004;103(4):1333-1341.

20. Auger JM, Kuijpers MJ, Senis YA, Watson SP, Heemskerk JW. Adhesion of human and mouse platelets to collagen under shear: a unifying model. FASEB J. 2005;19(7):825-827.

21. Eckly A, Rinckel JY, Proamer F, et al. Respective contributions of single and compound granule fusion to secretion by activated platelets. Blood. 2016;128(21):2538-2549.

22. Joshi S, Whiteheart SW. The nuts and bolts of the platelet release reaction. Platelets. 2017;28(2):129137.

23. Cattaneo $\mathrm{M}$. The platelet $\mathrm{P} 2 \mathrm{Y}_{12}$ receptor for adenosine diphosphate: congenital and drug-induced defects. Blood. 2011;117(7):2102-2112.

24. Jones S, Evans RJ, Mahaut-Smith MP. Ca ${ }^{2+}$ influx through P2X1 receptors amplifies $\mathrm{P} 2 \mathrm{Y} 1$ receptor-evoked $\mathrm{Ca}^{2+}$ signaling and ADP-evoked platelet aggregation. Mol Pharmacol. 2014;86(3):243-251.

25. Cosemans JM, Iserbyt BF, Deckmyn H, Heemskerk JW. Multiple ways to switch platelet integrins on and off. J Thromb Haemost. 2008;6(8):1253-1261.

26. de Witt SM, Swieringa F, Cavill R, et al. Identification of platelet function defects by multi-parameter assessment of thrombus formation. Nat Commun. 2014;16(5):4257.

27. Mastenbroek TG, Feijge MA, Kremers RM, et al. Platelet-associated matrix metalloproteinases regulate 
thrombus formation and exert local collagenolytic activity. Arterioscler Thromb Vasc Biol. 2015;35(12):25542561.

28. Fong KP, Barry C, Tran AN, et al. Deciphering the human platelet sheddome. Blood. 2011;117(1):e15-e26. 29. Gardiner EE, Karunakaran D, Shen Y, et al. Controlled shedding of platelet glycoprotein (GP)VI and GPIbIX-V by ADAM family metalloproteinases. J Thromb Haemost. 2007;5(7):1530-1537.

30. Dreymueller D, Pruessmeyer J, Groth E, Ludwig A. The role of ADAM-mediated shedding in vascular biology. Eur J Cell Biol. 2012;91(6-7):472-485.

31. Gardiner EE, Andrews RK. Platelet receptor expression and shedding: glycoprotein Ib-IX-V and glycoprotein VI. Transfus Med Rev. 2014;28(2):56-60.

32. Jansen AJ, Josefsson EC, Rumjantseva $\mathrm{V}$, et al. Desialylation accelerates platelet clearance after refrigeration and initiates GPIba metalloproteinase-mediated cleavage in mice. Blood. 2012;119(5):12631273.

33. Munnix IC, Cosemans JM, Auger JM, Heemskerk JW. Platelet response heterogeneity in thrombus formation. Thromb Haemost. 2009;102(6):1149-1156.

34. Heemskerk JW, Mattheij NJ, Cosemans JM. Platelet-based coagulation: different populations, different functions. J Thromb Haemost. 2013;11(1):2-16.

35. Munnix IC, Kuijpers MJ, Auger JM, et al. Segregation of platelet aggregatory and procoagulant microdomains in thrombus formation: regulation by transient integrin activation. Arterioscler Thromb Vasc Biol. 2007;27(11):2484-2490.

36. Jackson SP, Schoenwaelder SM. Procoagulant platelets: are they necrotic? Blood. 2010;116(12):20112018.

37. Bevers EM, Comfurius P, van Rijn JL, Hemker HC, Zwaal RF. Generation of prothrombin-converting activity and the exposure of phosphatidylserine at the outer surface of platelets. Eur J Biochem. 1982;122(2):429436.

38. van Kruchten R, Mattheij NJ, Saunders C, et al. Both TMEM16F-dependent and TMEM16F-independent pathways contribute to phosphatidylserine exposure in platelet apoptosis and platelet activation. Blood. 2013;121(10):1850-1857.

39. Lhermusier T, Chap H, Payrastre B. Platelet membrane phospholipid asymmetry; from the characterization of a scramblase activity to the identification of an essential protein mutated in Scott syndrome. J Thromb Haemost. 2011;9(10):1883-1891.

40. Berny MA, Munnix IC, Auger JM, et al. Spatial distribution of factor Xa, thrombin, and fibrin(ogen) on thrombi at venous shear. PLoS One. 2010;5(4):e10415.

41. Ruf W, Riewald M. Regulation of tissue factor expression: Landes Bioscience; 2000-2013.

42. Mackman N, Tilley RE, Key NS. Role of the extrinsic pathway of blood coagulation in hemostasis and thrombosis. Arterioscler Thromb Vasc Biol. 2007;27(8):1687-1693.

43. Hoffman M, Monroe DMr. A cell-based model of hemostasis. Thromb Haemost. 2001;85(6):958-965.

44. Alberio L, Ravanat C, Hechler B, et al. Delayed-onset of procoagulant signalling revealed by kinetic analysis of COAT platelet formation. Thromb Haemost. 2017;117(6):1101-1114.

45. Mattheij NJ, Swieringa F, Mastenbroek TG, et al. Coated platelets function in platelet-dependent fibrin formation via integrin $\alpha$ llb $\beta 3$ and transglutaminase factor XIII. Haematologica. 2016;101(4):427-436.

46. Vandendries ER, Hamilton JR, Coughlin SR, Furie B, Furie BC. Par4 is required for platelet thrombus propagation but not fibrin generation in a mouse model of thrombosis. Proc Natl Acad Sci U $S$ A. 2007;104(1):288-292.

47. Stalker TJ, Welsh J, Tomaiuolo M, et al. A systems approach to hemostasis: 3 . Thrombus consolidation regulates intrathrombus solute transport and local thrombin activity. Blood. 2014;124(11):1824-1831.

48. Ten Berg JM, Zwart B, van ' $t$ Hof AW, et al. Optimal duration of dual antiplatelet therapy after percutaneous coronary intervention or after acute coronary syndrome: Practical lessons from a review. Neth Heart J. 2017; Epub ahead of print.

49. Steg PG, James SK, Atar D, et al. ESC Guidelines for the management of acute myocardial infarction in patients presenting with ST-segment elevation. Eur Heart J. 2012;33(20):2569-2619.

50. Floyd CN, Passacquale G, Ferro A. Comparative pharmacokinetics and pharmacodynamics of platelet adenosine diphosphate receptor antagonists and their clinical implications. Clin Pharmacokinet. 2012;51(7):429-442.

51. Schrör K. Why we should not skip aspirin in cardiovascular prevention. Hamostaseologie. 2016;36(1):3343. 
52. Ho PM, Tsai TT, Wang TY, et al. Adverse events after stopping clopidogrel in post-acute coronary syndrome patients: Insights from a large integrated healthcare delivery system. Circ Cardiovasc Qual Outcomes. 2010;3(3):303-308.

53. Mylotte D, Peace AJ, Tedesco AT, et al. Clopidogrel discontinuation and platelet reactivity following coronary stenting. J Thromb Haemost. 2011;9(1):24-32.

54. Charlot M, Nielsen LH, Lindhardsen J, et al. Clopidogrel discontinuation after myocardial infarction and risk of thrombosis: a nationwide cohort study. Eur Heart J. 2012;33(20):2527-2534.

55. Mauri L, Kereiakes DJ, Yeh RW, et al. Twelve or 30 months of dual antiplatelet therapy after drug-eluting stents. N Engl J Med. 2014;371(23):2155-2166.

56. Desborough MJ, Smethurst PA, Estcourt LJ, Stanworth SJ. Alternatives to allogeneic platelet transfusion. Br J Haematol. 2016;175(3):381-392.

57. van der Meer PF. PAS or plasma for storage of platelets? A concise review. Transfus Med. 2016;26(5):339342.

58. Apelseth TO, Hervig T, Bruserud O. Current practice and future directions for optimization of platelet transfusions in patients with severe therapy-induced cytopenia. Blood Rev. 2011;25(3):113-122.

59. Woodcock BE, Cooper PC, Brown PR, et al. The platelet defect in acute myeloid leukaemia. J Clin Pathol. 1984;37(12):1339-1342.

60. Leinoe EB, Hoffmann MH, Kjaersgaard E, Johnsen HE. Multiple platelet defects identified by flow cytometry at diagnosis in acute myeloid leukaemia. Br J Haematol. 2004;127(1):76-84.

61. Kozek-Langenecher S. Fluids and coagulation. Curr Opin Crit Care. 2015;21(4):285-291.

62. Bollinger D, Görlinger K, Tanaka KA. Pathophysiology and treatment of coagulopathy in massive hemorrhage and hemodilution. Anesthesiology. 2010;113(5):1205-1219.

63. Kozek-Langenecher SA, Ahmed AB, Afshari A, et al. Management of severe perioperative bleeding: guidelines from the European Society of Anaesthesiolofy: First update 2016. Eur J Anaesthesiol. 2017;34(6):332-395.

64. Jirouskova $M$, Shet AS, Johnson GJ. A guide to murine platelet structure, function, assays, and genetic alterations. J Thromb Haemost. 2007;5(4):661-669.

65. Westrick RJ, Winn ME, Eitzman DT. Murine models of vascular thrombosis (Eitzman series). Arterioscler Thromb Vasc Biol. 2007;27(10):2079-2093.

66. Jagadeeswaran P, Cooley BC, Gross PL, Mackman N. Animal models of thrombosis from zebrafish to nonhuman primates: Use in the elucidation of new pathologic pathways and the development of antithrombotic drugs. Circ Res. 2016;118(9):1363-1379.

67. van Kruchten R, Cosemans JM, Heemskerk JW. Measurement of whole blood thrombus formation using parallel-plate flow chambers - a practical guide. Platelets. 2012;23(3):229-242.

68. Nagy M, Heemskerk JW, Swieringa F. Use of microfluidics to assess the platelet-based control of coagulation. Platelets. 2017; Epub ahead of print:1-8. 




\section{Chapter 2}

Platelet populations and priming in hematological diseases

Baaten CC, Ten Cate H, van der Meijden PE*, Heemskerk JW* (*equal contribution)

Blood Rev. 2017; 31(6):389-399

Reprinted with permission 


\section{Abstract}

In healthy subjects and patients with hematological diseases, platelet populations can be distinguished with different response spectra in hemostatic and vascular processes. These populations partly overlap, and are less distinct than those of leukocytes. The platelet heterogeneity is linked to structural properties, and is enforced by inequalities in the environment. Contributing factors are variability between megakaryocytes, platelet ageing, and positive or negative priming of platelets during their time in circulation. Within a hemostatic plug or thrombus, platelet heterogeneity is enhanced by unequal exposure to agonists, with populations of contracted platelets in the thrombus core, discoid platelets at the thrombus surface, patches of ballooned and procoagulant platelets forming thrombin, and coated platelets binding fibrin. Several pathophysiological hematological conditions can positively or negatively prime the responsiveness of platelet populations. As a consequence, in vivo and in vitro markers of platelet activation can differ in thrombotic and hematological disorders.

\section{Introduction}

Individual platelets interact in numerous ways with the vessel wall or adherent blood cells. This versatility is fundamental to the role of platelets in a wide range of (patho)physiological processes, ranging from vascular repair, hemostasis and thrombosis, to inflammation progression, innate immunity and tumor metastasis. In the past years, evidence has been accumulating that circulating platelets are markedly heterogeneous in properties, which has led to the suggestion that identifiable populations of platelets with specialized response spectra are best suited for specific roles. In the present paper, we resume the current evidence for heterogeneity in terms of composition and functions of platelets during their formation from megakaryocytes, when circulating over time in the circulation, and once adhered to a vessel wall. We further describe how various physiological and pathophysiological conditions can change or prime the responsiveness of circulating platelets, and hence alter the distribution of platelet populations. We finally define how in vitro and in vivo markers of platelet activation phenotypes can be judged in relation to thrombotic and hematological disorders.

\section{Intrinsic factors of platelet heterogeneity}

Platelets from a given subject, healthy or diseased, greatly vary in receptor expression levels and markedly diverge in responsiveness once activated. Different populations of activated platelets can be distinguished, which can differently interact with the inflamed or injured vessel wall and differently support hematological processes. 
This heterogeneity is explained by several intrinsic factors, including variability of clonal megakaryocytes, unequal division of megakaryocyte-derived proplatelets, and modifications upon ageing of the newly formed platelets.

\subsection{Heterogeneity between megakaryocytes and platelets}

Heterogeneity between megakaryocytes, whether or not linked to a specific niche in the bone marrow or lungs, is a likely cause of inter-platelet variability, although there is only limited literature available on this subject. Several authors have described that polyploid megakaryocytes, either cultured from $\mathrm{CD} 34^{+}$hematopoietic stem cells or derived from immortalized cell lines, considerably differ from cell to cell, in terms of levels of cytoplasmic and membrane proteins, as well as in agonist-induced $\mathrm{Ca}^{2+}$ transients. ${ }^{1-3}$ Recently, this was demonstrated also for megakaryocytes derived from single-cell clones of forward-programmed human pluripotent stem cells. After re-programming, individual

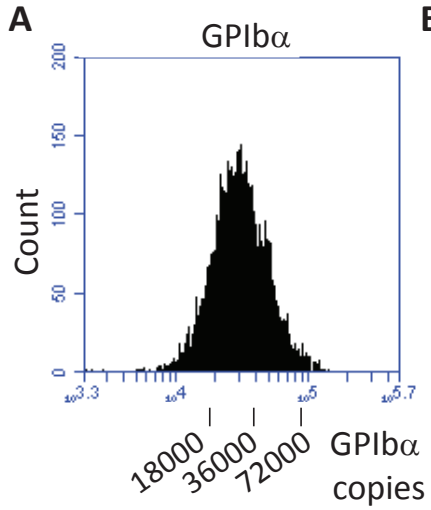

C

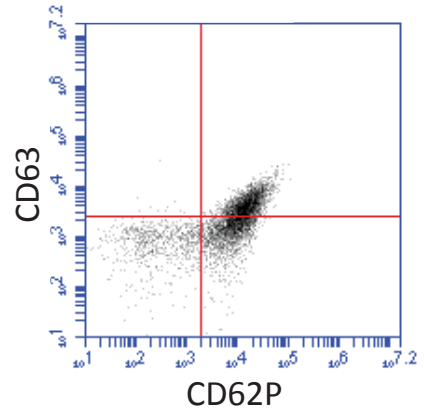

B

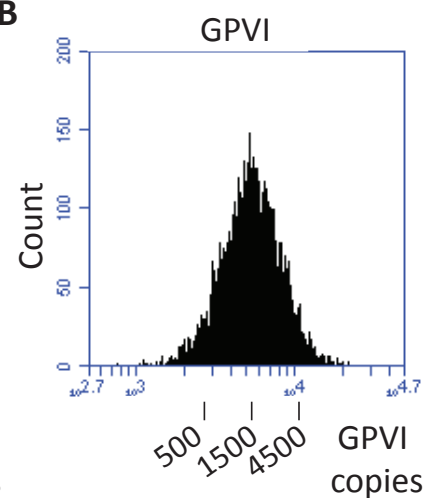

D

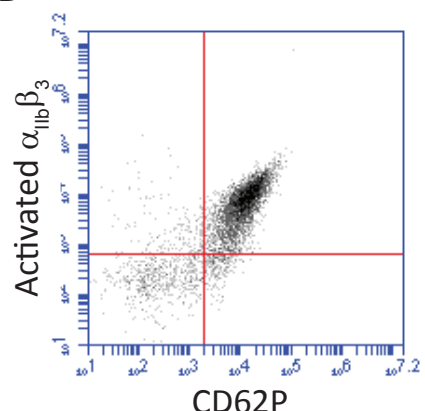

E

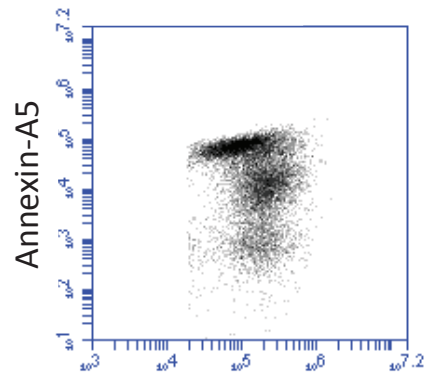

FSC

Figure 1. Intrinsic heterogeneity in platelet composition and functions. (A, B) Differential expression of key adhesive receptors in the total platelet population, immunologically stained for GPIb $\alpha$ or GPVI. Indicated in the flow cytometric histograms are the estimated copy numbers, based on proteome analysis. ${ }^{128}$ (C, D) Platelets stimulated with a GPVI agonist immunologically stained for CD62P in combination with CD63; or CD62P in combination with a marker for activated integrin $\alpha_{11 b} \beta_{3}$. Shown is the presence of different platelet populations with alpha granule secretion $\left(C D 62 \mathrm{P}^{+}\right)$with or without dense granule secretion or $\alpha_{\| 1 b} \beta_{3}$ activation. (E) Two apoptotic platelet populations after stimulation with BH3 mimetic ABT-737, displaying low or high phosphatidylserine (PS) exposure. 
megakaryocytes of the same clone showed a 100-fold difference in expression levels of common receptor proteins, such as glycoprotein (GP)lba, GPVI and integrin $\alpha_{1 \mid b} \beta_{3}$, as assessed by flow cytometry. ${ }^{4}$ No studies are yet available to demonstrate whether distinct megakaryocytes (in the bone marrow) also yield different types of platelets. However, one can expect that single platelets 'inherit' at least in part their expression profiles of receptors and other signaling molecules from the precursor megakaryocyte. It has been shown that when the environment of megakaryocytes changes (e.g. upon inflammation or diabetes), the transcriptome of platelets is influenced as well., ${ }^{5,}$ For instance, patients diagnosed with the autoimmune disease systemic lupus erythematosus (SLE) may have an altered platelet transcriptome. The changes in platelet mRNA and protein levels were linked to increased procoagulant activity and platelet-monocyte interactions in SLE patients. $^{7}$

Proplatelets are formed from the demarcation compartment of megakaryocytes by pinching off the cytosol containing cell membrane compartment and organelles. ${ }^{8}$ Although studies are lacking, it can be conceived that part of the heterogeneity between platelets may come from unequal retention or re-distribution of the cellular components from the mother cell, including surface receptors, actin and tubulin cytoskeletons, signalosomes, internal membrane vesicles, secretory granules, ribosomes, and mitochondria.

Clear evidence for heterogeneity in platelet size and volume comes from flow cytometric profiles and blood count histograms. Forward side scatter plots (flow cytometry) point to a considerable variation in size, which is in agreement with the relative large distribution width of the platelet volume (blood cell count). Sizable heterogeneity between platelets is also detectable by quantifying the abundantly expressed receptors. Thus, flow cytometric profiles of platelets that are stained with fluorescent antibodies against integrin $\beta_{3}$ (CD61) point to a large inter-platelet variation in expression levels of this integrin. ${ }^{9,10}$ Similarly, staining for GPIb $\alpha$ or GPVI gives histograms, which show an about 10-fold difference in fluorescence intensity (indicative of expression level) of platelets in the lower and upper 10\% percentiles (Figure 1A-B). Evidence for signaling heterogeneity comes from recordings of the $\mathrm{Ca}^{2+}$ responses of single platelets from an arbitrary donor, which appear to vary greatly in the type of transient $\mathrm{Ca}^{2+}$ fluxes after stimulation via ADP, thrombin or collagen receptors. ${ }^{11}$

\subsection{Populations of activated platelets}

Platelets, upon activation by agonists, are known to form populations with different surface properties..$^{10}$ For instance, platelets stimulated with a maximal dose of a GPVI agonist showing CD62P expression (marker of $\alpha$-granule secretion) only display CD63 expression to a certain degree (marker of $\delta$-granule and lysosome release), while the majority of platelets that express CD62P do have activated integrin $\alpha_{\| b} \beta_{3}$ (Figure 1CD). Inter-platelet heterogeneity in granule release has also been observed by electron microscopy. ${ }^{12}$ Thus, secretion of granule content can occur in two different ways: single granule secretion (both $\alpha$ and $\delta$ granules), or secretion of multiple granules fused into one large compartment (mainly $\alpha$ granules). ${ }^{12}$ Dependent on the strength of an activation trigger, secretion of the granule content appears to be regulated differently and can 
result in different secretion patterns (Figure 1C-D) ${ }^{12,13}$ Another consistent observation is that, regardless of the agonist used, a fraction of $10-20 \%$ of the platelets fails to stain for $\mathrm{CD} 62 \mathrm{P}^{14}{ }^{14}$ suggesting that some platelets are refractory to $\alpha$-granule secretion.

A striking heterogeneity is observed when platelets in suspension are stimulated with combinations of collagen and thrombin receptor agonists. In addition to an population showing integrin $\alpha_{11 b} \beta_{3}$ activation and granule secretion, another population of swollen platelets appears with exposed phosphatidylserine at their outer membrane surface, inactivated/closed integrins, and capability of coagulation factor binding. ${ }^{15,16}$ Depending on the activating conditions, the majority of the latter platelets also have a transglutaminase-dependent fibrin coat at their surface, ${ }^{17}$ which is in agreement with the early description of coated or COAT platelets. ${ }^{18}$ As described below, the various platelet populations are considered to have different roles, in supporting platelet aggregation, procoagulant activity and fibrin formation. The formation of phosphatidylserineexposing, procoagulant platelets is linked to a prolonged, high cytosolic $\mathrm{Ca}^{2+}$ level ${ }^{19,20}$ i.e. a condition required for swelling and phospholipid scrambling via the $\mathrm{Ca}^{2+}$-dependent anoctamin- 6 channel (gene ANO6 or TMEM16F). ${ }^{21,22}$ It can be speculated that, in single platelets, differences in both receptor expression levels and in activity of the $\mathrm{Ca}^{2+}$-flux machinery determine the formation of the platelet populations.

Populations of platelets with different surface properties are also formed after stimulation with the proapoptotic BH3 mimetic ABT-737. ${ }^{23}$ Typically, platelets with low and high phosphatidylserine exposure are formed in this case (Figure 1E).

\subsection{Changes with platelet maturation, ageing and apoptosis}

Early studies supposed that platelet activity correlates with platelet size and, hence, receptor and granule numbers. ${ }^{24,25}$. Later papers have focused on platelet age, considering that newly formed platelets are larger in size and therefore more active than older platelets. ${ }^{26,27}$ However, there is still not much support for the idea that platelet size is the only or main factor determining platelet responsiveness to agonists. Association studies do not suggest such a relation. For instance, in a cohort of healthy individuals, mean platelet volume negatively correlated with the amount of newly formed platelets, suggesting that young platelets are not necessarily the largest ones. ${ }^{28}$

A consistent finding is that newly formed platelets are enriched in mRNA, as observed by specific mRNA-staining and cell counting analyses. ${ }^{29}$ Young platelets with detectable mRNA in the cytoplasm have been termed as reticulated platelets ${ }^{30}$, ${ }^{31}$ or immature platelet fraction. ${ }^{32}$ The latter fraction, with a normal reference range of $1-5 \%$, is considered to reflect the rate of platelet production in the bone marrow. Given that platelets have overall lost $>90 \%$ of the mRNA of their progenitor cells while retaining the stable circular RNAs,$-^{33}$ it is clear that the residual mRNA levels in reticulated platelets reflect incomplete mRNA maturation. Interestingly, subject age does not seem to be an important modifier of reticulated platelets, as in older subjects only $\sim 2 \%$ of all $>6000$ identified mRNA and miRNA species were found to be changed in expression levels. ${ }^{34}$ On the other hand, a Spanish hemophilia study showed that the reticulated platelet fraction has a high heritability component..$^{35}$ Recent papers describe that certain combinations of mRNA and miRNA correlate with platelet phenotype, 
raising the possibility to use these RNA species as biomarkers of platelet function. ${ }^{36,37}$

With a circulation time of 7-10 days and a remarkably constant platelet count over time, the daily turnover (production and removal) of platelets is extremely well regulated, being estimated at about $10^{11}$ platelets per day. ${ }^{8,38}$. Platelet removal from the circulation occurs via both clearance and "consumption" (at the enormous vascular bed in the human body). Clearance can be triggered by prior platelet activation and an apoptotic process, e.g. resulting in the surface exposure of $\mathrm{PS}^{39}$ or by other still unknown causes.

Evidence for a link between platelet ageing and apoptosis comes from experiments with mice, lacking the pro-apoptotic proteins Bax/Bak, in which platelet survival is greatly extended. ${ }^{40}$ Because aged, Bak-deficient platelets showed a reduced capability of thrombin- and collagen-induced secretion, these were designated as exhausted platelets. ${ }^{41}$ On the other hand, in mice lacking the anti-apoptotic protein $\mathrm{BCl}-\mathrm{xL}$, platelet lifetime was shortened and platelet function was also reduced..$^{40}$ The interpretation was that a loss of $\mathrm{BCl}-\mathrm{xL}$ leads to activation of Bax/Bak to induce a proapoptotic condition. In patients with malignancies, who were treated with the $\mathrm{Bcl}-\mathrm{xL}$ degrading drug Navitoclax (a BH-3 mimetic also known as ABT-263), platelet lifetime was also shortened, thus resulting in mild thrombocytopenia. ${ }^{39}$ Characteristics of apoptotic platelets are mitochondrial dysfunction, caspase proteolytic activity, refractoriness to integrin activation, and surface exposure of phosphatidylserine. ${ }^{42,43}$

Another finding was that aged platelets gradually loose sialic acid residues from their surface membrane glycoproteins. ${ }^{38}$ Desialylated platelets were found to be recognized by Ashwell-Morell receptors in the liver, mediating platelet clearance and, interestingly, also mediating release of thrombopoietin, which is the main cytokine regulating platelet formation from megakaryocytes. ${ }^{44}$ These data are suggestive for a gradual, ongoing formation of the population of (pro)apoptotic platelets.

\section{Environmental factors of platelet heterogeneity}

Platelets can interact with a large variety of glycoproteins in the inflamed or damaged vessel wall. The adhesive interactions include: activated endothelial cells expressing von Willebrand factor (VWF); subendothelial matrix components such as laminins, collagens, thrombospondins; adhered plasma proteins, such as VWF, fibrin(ogen), fibronectin and vitronectin; leukocytes under inflammatory conditions; lymphatic wall components like podoplanin; tumor cells; and other platelets via fibrinogen bridges. Given that these adhesive ligands all induce different signaling pathways, the response of an adhered platelet will vary with the precise location and substrate of adhesion. Well studied is the environmental directed heterogeneity in responses of platelets adhering to collagen and assembling into a thrombus. ${ }^{10,16}$ The formation of platelet populations in thrombus formation is further stimulated by the local generation of thrombin. ${ }^{45,46}$

\subsection{Platelet heterogeneity upon adhesion and activation}

Platelets will respond differently, when interacting with specific vascular wallderived proteins. This response heterogeneity is studied most extensively for platelets adhering to fibrillar collagen via the receptors GPVI and integrin $\alpha_{2} \beta_{1} \cdot{ }^{16,47}$ Both receptors 

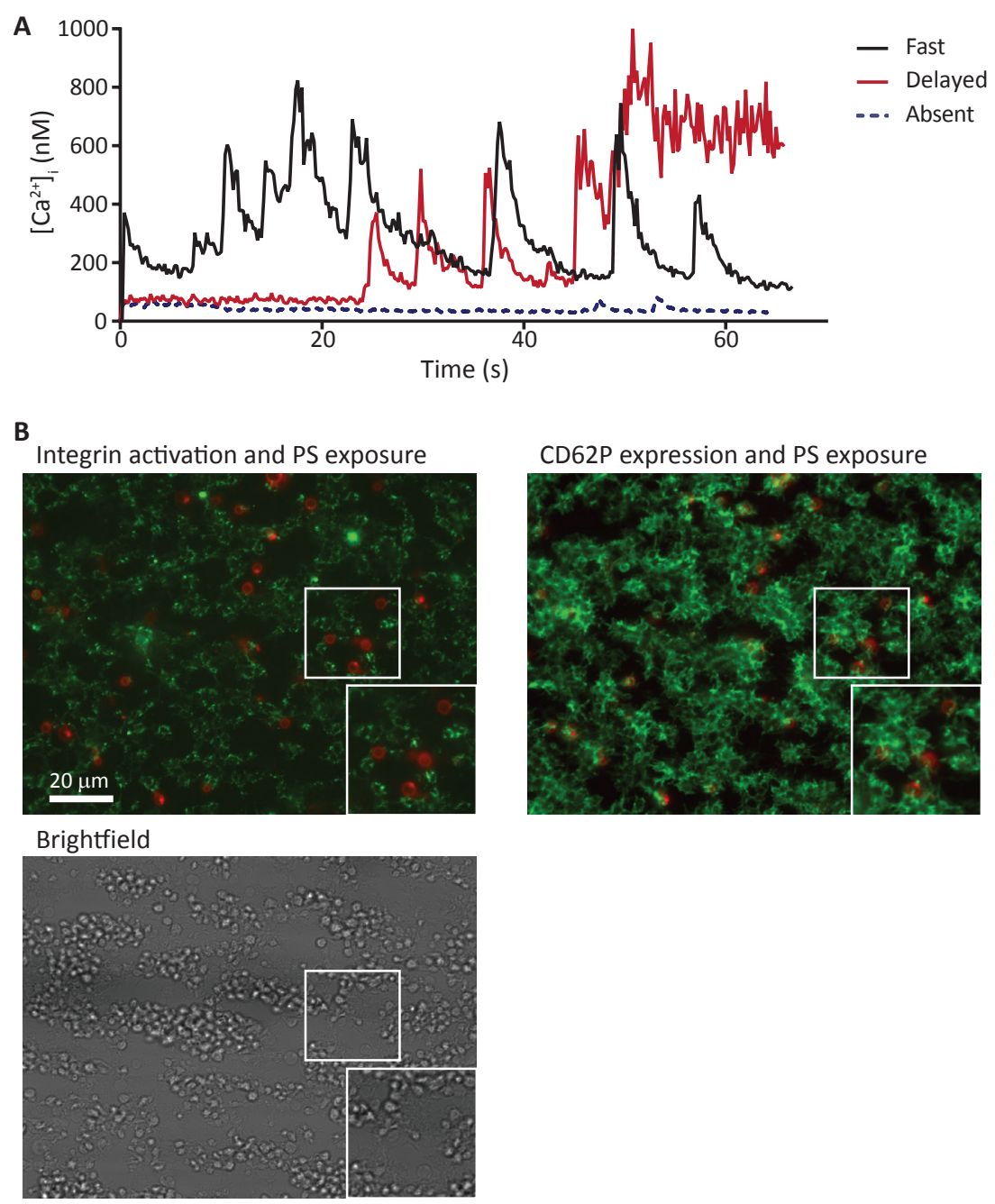

Figure 2. Heterogeneity of platelet responses. (A) Distinct calcium fluxes of single platelets adhered under flow to collagen. Based on the time between adhesion and increase in calcium signal, platelets were classified as having a fast, delayed or absent activation response. (B) Platelet populations in thrombi formed on collagen: aggregating platelets characterized by integrin activation and CD62P expression (green) and procoagulant platelets forming blebs/balloons and exposing PS (red).

act in synergy to immobilize and thereby activate platelets via the GPVI signalosome, resulting in $\mathrm{Ca}^{2+}$ elevation, integrin activation and granular secretion. ${ }^{48,49}$ Collagen fibrils allowing platelet adhesion to both receptors are most strongly activating, and form the largest platelet aggregates. A population of the collagen-adhered platelets subjected to the highest $\mathrm{Ca}^{2+}$ fluxes -, responds by surface exposure of procoagulant phosphatidylserine..$^{50}$ This so-called procoagulant response is accomplished via the ion channel anoctamin- 6,22 and accompanied by marked morphological changes involving 
swelling, bleb and balloon formation. ${ }^{51,52}$ Phosphatidylserine exposure facilitates the binding of multiple coagulation factors and promotes thrombin generation. ${ }^{19}, 20$ Remarkably, the majority of collagen-bound platelets is refrained from phosphatidylserine exposure and swelling.

\subsection{Platelet heterogeneity within a thrombus}

Heterogeneity in platelet activation properties progresses during collagendependent thrombus formation in flowing blood, both in vitro and in vivo. At high, arterial wall-shear rates, platelet adhesion to collagen/VWF is accomplished via GPVI, integrin $\alpha_{2} \beta_{1}$ and the VWF receptor, GPIb-V-IX. ${ }^{53,54}$ Single-cell studies of adhered platelets from flowing blood again show marked differences in $\mathrm{Ca}^{2+}$ fluxes (Figure 2A)..$^{55}$ Thrombi formed under high-shear flow show clusters of aggregated platelets (staining for activated integrin $\alpha_{\| 1 \mathrm{~b}} \beta_{3}$ and P-selectin), alternated with patches of ballooned phosphatidylserine-exposing platelets (binding annexin A5 and coagulation factors) (Figure 2B). ${ }^{56,57}$ The population of aggregated platelets also undergoes contraction. ${ }^{58,59}$ It is not quite clear how platelets 'decide' to either aggregate or expose phosphatidylserine. Given that adjacent platelets can form gap junctions, ${ }^{60}$ and communicate with each other, ${ }^{61}$ it is possible that crosscommunication in the aggregate protects against anoctamin-6-dependent procoagulant activity. In a study where platelet adhesion to multiple surfaces was examined, the response heterogeneity in thrombus formation with different populations of platelets formed was a constant finding, regardless of the surface type and adhesive receptors involved (GPIb-V-IX, GPVI, CLEC- $2, \alpha_{6} \beta_{1}, \alpha_{110} \beta_{3}$ or CD36). ${ }^{62}$

Recent in vivo studies with mice, where laser-induced injury was applied to cremaster or femoral arterioles, have pointed to another level of platelet heterogeneity within thrombi, i.e. an inner core of aggregated and contracted platelets, surrounded by an outer shell of loosely adhered platelets. ${ }^{63,}{ }^{64}$ Two specific platelet populations were distinguished, i.e. platelets in the thrombus core having undergone secretion and CD62P expression, and platelets in the outer shell without secretion. ${ }^{63,64}$ Several factors may contribute to the heterogeneous thrombus buildup, including local levels of soluble platelet agonists (ADP, thromboxane $A_{2}$ ) and the local wall shear gradients. ${ }^{65}$ Also, this type of platelet heterogeneity in thrombi is likely to be influenced by the type of injury, vascular bed location and the physiological or pathological state of the vessel.

\subsection{Coagulation and thrombus heterogeneity}

Under conditions favoring coagulation, e.g. with tissue factor present, the formation of thrombin and fibrin in a thrombus appear to be highly localized processes. In mouse microvascular thrombosis models, thrombin activity and fibrin deposits appeared to be concentrated at the thrombus base, i.e. close to the vascular localization of tissue factor. ${ }^{66}$ In vitro models of thrombus formation showed that the population of phosphatidylserine-exposing platelets was responsible for the binding of coagulation factors (prothrombin, factors Va, IXa and Xa), and also for the formation of thrombin and fibrin. ${ }^{46,57} \mathrm{~A}$ potent feedback loop was identified, in that the local formation of thrombin enhanced thrombin-mediated phosphatidylserine exposure of nearby platelets, thus increasing the population of procoagulant platelets. ${ }^{46}$ Fibrin formation appeared to be 


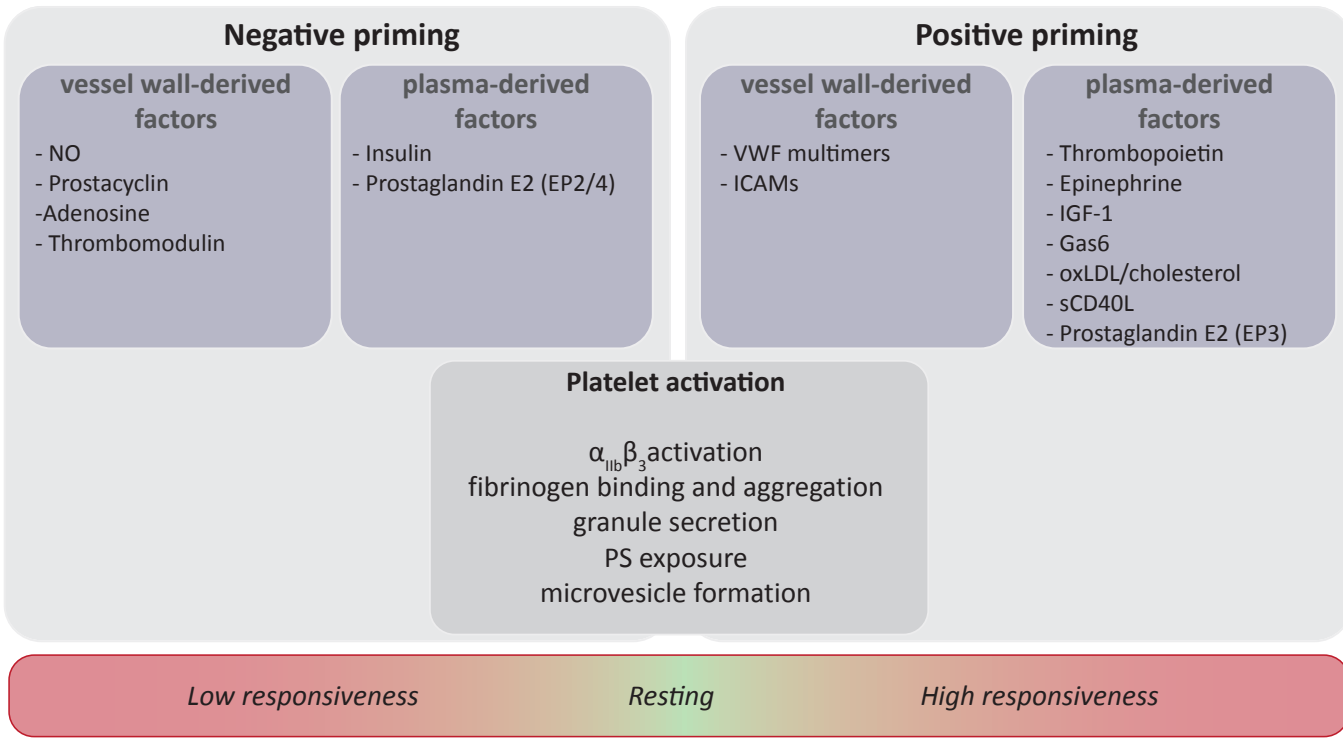

Figure 3. Concept of negative and positive priming of platelets. The indicated factors can cause negative or positive priming of platelet activation processes, either plasma-derived or from the vessel wall.

initially linked to PS exposure, and secondarily dependent on integrin $\alpha_{11 b} \beta_{3}$ activation and transglutaminase-dependent fibrin cross-linking. ${ }^{17,67}$ In vitro models further indicated that fibrin preferentially localized near the sites of tissue factor and on procoagulant (coated) platelets. ${ }^{68}$ Other identified elements of heterogeneity in a thrombus are the local secretion of polyphosphate clusters (activating factor $\mathrm{XII})^{69}$, and the phosphatidylserinedependent accumulation of plasmin (activating the fibrinolytic system)..$^{70}$ Taken together, during thrombus formation, the coagulation process appears to enhance the formation of distinct platelet populations, and increase the heterogeneity in platelet functions.

\section{Positive and negative priming of platelets}

Multiple bioactive molecules in the blood can enhance or suppress platelet functionality. In psychological and neurobiological sciences, the implicit memory effect by which exposure to the first stimulus influences the response to the next stimulus, is called 'priming'. ${ }^{71}$ By analogy, we can consider the exposure of platelets to enhancing or suppressing agents as positive or negative priming events. Figure 3 gives an illustration of potential priming biomolecules that are either derived from the vascular wall or found in plasma.

\subsection{Positive priming: key roles of plasma derived factors}

Several hormones enhance platelet activation processes (shape change, secretion, aggregation), when applied in combination with other agonists. Epinephrine, binding to the Gi-coupled $\alpha_{2}$-adrenergic receptor, reduces intracellular cyclic AMP levels which, 
e.g. enhances $\mathrm{Ca}^{2+}$ fluxes raised by agonists like ADP and thrombin. ${ }^{16,72}$ Thrombopoietin, a growth factor for megakaryocytes, also sensitizes platelets to agonists at threshold concentrations, ${ }^{73,74}$ and at shear stress. ${ }^{75}$ Similarly, insulin-like growth factor-1 (IGF1) greatly enhances platelet activation provided that other agonists are present. ${ }^{76}$ In vitro studies indicate that positive priming of platelets with thrombopoietin or insulin-like growth factor- 1 can overcome the inhibitory effects on platelet function by aspirin or $\mathrm{P}^{2} \mathrm{Y}_{12}$ receptor treatment. ${ }^{77}$ This suggests that positive priming of platelets can be a mechanism of resistance to antiplatelet therapy.

Other positive priming molecules are Gas6 (plasma-derived) and soluble CD40L (platelet-derived), both of which stabilize and extend platelet aggregation. ${ }^{78,79} \mathrm{TNF} \alpha$, a pro-inflammatory cytokine which is synthesized in high amounts during inflammatory conditions, is capable of inducing platelet activation mainly indirectly via endothelial activation, ${ }^{80}$ although some authors report an enhancing effect of TNF $\alpha$ on collageninduced aggregation. ${ }^{81}$

Besides the positive enforcement of such plasma molecules on platelet function, wall shear stress is of great influence as well. Rapid changes in laminar blood flow and shear rate, which occur at sites of stenosis, promote platelet aggregate formation. ${ }^{58,}$ 65,82 This wall-shear effect is highly dependent on VWF activity and autocrine platelet stimulation. ${ }^{65}$ As such, disturbances in blood flow can be considered as a positive priming condition.

Several dietary nutrients are known to positively modulate platelet responsiveness. In addition, gut microbes may contribute to platelet hyperreactivity via the generation of trimethylamine $\mathrm{N}$-oxide (TMAO) from dietary sources of trimethylamine, present in a western diet. The generated TMAO is proposed to prime platelet activation by enhancing $\mathrm{Ca}^{2+}$ release from intracellular stores. ${ }^{83}$

\subsection{Negative priming: key roles of endothelial mediators}

Not all platelets are freely circulating, as part of the platelets are kept in the spleen and lungs. ${ }^{84,85}$ In mice, the lungs also significantly contribute to platelet biogenesis. ${ }^{86}$ Whether this also applies to the human situation, remains to be explored. Clearly, these pools of marginated platelets in the lungs and spleen are exposed to different environments. Unclear is at which rate and to what extent the platelet pools exchange, as well as how these different locations alter the activation properties. Adaptions in properties can also arise when platelets are flowing through the micro- and macrovascular beds lined with specialized endothelial cells.

At least three endothelial-derived substances - likely there are more - are accountable for the major negative priming effect of the vessel wall on platelet activation. These are prostacyclin, nitric oxide and CD39/CD73. ${ }^{87}$ Prostacyclin (prostaglandin $\mathrm{I}_{2}$ ), a short-lived eicosanoid produced by endothelial cells, binds to the platelet IP receptor, which couples to the Gs GTP-binding protein, stimulates adenylate cyclase and thereby elevates the inhibitory second messenger cyclic AMP. ${ }^{88}$ Continuous exposure of circulating platelets to prostacyclin leads to cyclic AMP elevation and thereby platelet inhibition. In freshly isolated platelets, supra-basal levels of cyclic AMP have indeed been measured. ${ }^{72}$ However, IP receptors can desensitize and the functional effects of prostacyclin on 
platelets may be most prominent locally, i.e. after platelet adhesion. ${ }^{87}$ Prostaglandin $E_{2}$ (EP receptor agonist), also has an inhibitory though more complex action mechanism..$^{88}$ The effect of $P G E_{2}$ depends on the receptor isotype that is activated. Activation of EP3 isoforms by PGE 2 leads to sensitization of platelets, while binding of PGE ${ }_{2}$ to EP2 or EP4 receptors causes platelet inhibition. It has been proposed that specifically the blocking of EP3 could be beneficial for the prevention of atherothrombosis. ${ }^{89}$

The unstable free radical nitric oxide is membrane-permeable, and interacts with intracellular guanylate cyclase, raising the inhibitory second messenger cyclic GMP in platelets..$^{90}$ Nitric oxide is produced by endothelial nitric oxide synthase isoforms in response to the pulsatile flow of blood. Together, elevated levels of cyclic AMP and cyclic GMP accomplish strong platelet inhibition via a flood of protein phosphorylation reactions, mediated by the protein kinases $A$ and $G$, respectively. ${ }^{91,92}$ The levels of both cyclic nucleotides are balanced via cyclic nucleotide-dependent phosphodiesterases. The hormone insulin can also affect platelet reactivity via cyclic AMP and GMP elevation. In diabetic patients, who are resistant to insulin, a loss of these inhibitory pathways might contribute to platelet hyperreactivity. ${ }^{93,94}$

The endothelial-expressed ecto-nucleotidases CD39 and CD73 degrade intravascular ATP/ADP and AMP, respectively, to ultimately produce adenosine. ${ }^{87}$ These activities convert potent platelet-activating autacoids (ATP, ADP) into a platelet inhibitor (adenosine, activating Gs). ${ }^{87}$ Both mouse and human studies point towards a modulating role of ecto-nucleotidases in thrombus formation and thrombosis. In addition to the inhibitory $A_{2}$-adenosine receptor, platelets possess receptors linked to protein-tyrosine phosphatases, which also suppress activation processes..$^{95}$ The physiological ligands of these are mostly unknown. Indirectly, also the thrombin-inactivating activity of endothelial thrombomodulin contributes to platelet suppression, i.e. by preventing thrombin-induced activation..$^{96}$ Some nutritional components can contribute to negative priming, e.g. a mild to moderate alcohol consumption may lower platelet reactivity by inhibiting thromboxane formation. ${ }^{97}$

Given that circulating platelets are exposed to a plethora of positive and negative priming molecules, their net effect must be prevention of platelet adhesion and activation, unless positive stimuli prevail, such as at a damaged vessel wall. The implicit assumption is that a shift or disturbance in the balance of positive and negative priming molecules causes a change in the threshold of platelet activation. This could for example explain why the population of coated platelets increased in a variety of pathophysiological conditions. ${ }^{18}$

\section{Pathology induced alterations in platelet populations}

Considering that multiple bioactive mediators present in the blood or derived from the vessel wall influence the tendency of platelet activation, it can be expected that disease conditions associated with vascular abnormalities or blood changes enhance the positive priming of platelets. An increasing number of reports points to such increased priming events, often ascribed to the formation of different 'types' of platelets, especially in patients with high-burden inflammatory, prothrombotic or hematological diseases (Table 1). Albeit different terms are used to annotate the platelet changes, the majority of 
Table 1: Platelet phenotypes and activation markers in hematological and other diseases, induced by negative or positive priming. Representative examples from the literature are given.

\begin{tabular}{|c|c|c|c|c|c|}
\hline $\begin{array}{l}\text { Clinical } \\
\text { condition }\end{array}$ & $\begin{array}{l}\text { Hemostatic } \\
\text { balance }\end{array}$ & Type of plt & $\begin{array}{l}\text { Plt activation } \\
\text { markers in vivo }\end{array}$ & $\begin{array}{l}\text { Platelet } \\
\text { activation } \\
\text { testing in vitro }\end{array}$ & Refs \\
\hline \multicolumn{6}{|c|}{ Negatively primed (low responsive) } \\
\hline $\begin{array}{l}\text { Congenital } \\
\text { defects: Scott } \\
\text { syndrome }\end{array}$ & $\begin{array}{l}\text { mild bleed- } \\
\text { ing }\end{array}$ & procoagulant & n.d. & $\begin{array}{l}\text { PS } \downarrow \\
\text { fibrin formation } \\
\downarrow\end{array}$ & 22,68 \\
\hline $\begin{array}{l}\text { Hematological } \\
\text { malignancies }\end{array}$ & bleeding & $\begin{array}{l}\text { hypo-respon- } \\
\text { sive }\end{array}$ & $\begin{array}{l}\text { Plt count } \downarrow \\
\text { TPO } \uparrow\end{array}$ & $\begin{array}{l}\alpha \mathrm{llb} \beta 3, \mathrm{CD} 62 \mathrm{P}, \\
\mathrm{CD63}, \mathrm{GPIb} \downarrow\end{array}$ & $\begin{array}{l}100 \\
101\end{array}$ \\
\hline $\begin{array}{l}\text { Renal failure } \\
\text { and uremia }\end{array}$ & $\begin{array}{l}\text { bleeding } \\
\text { (thrombosis) }\end{array}$ & dysfunctional & NO 个 & $\begin{array}{l}\text { CD62P, Ca2+ } \downarrow \\
\text { PS, caspase } \uparrow\end{array}$ & $\begin{array}{l}102 \\
103\end{array}$ \\
\hline \multicolumn{6}{|c|}{ Positively primed (high responsive) } \\
\hline $\begin{array}{l}\text { Diabetes type } \\
2\end{array}$ & $\begin{array}{l}\text { prothrom- } \\
\text { botic }\end{array}$ & angry & $\begin{array}{l}\text { sCD62P, sCD40L, } \\
\text { PF4, MPV } \uparrow \\
\text { NO } \downarrow\end{array}$ & $\begin{array}{l}\text { GPIb, CD41, } \\
\text { GPVI } \uparrow \text { CD63, } \\
\text { CD62P, CAT }\end{array}$ & $\begin{array}{l}104 \\
105 \\
129 \\
130\end{array}$ \\
\hline $\begin{array}{l}\text { Hypercholes- } \\
\text { terolemia }\end{array}$ & $\begin{array}{l}\text { prothrom- } \\
\text { botic }\end{array}$ & hyper-reactive & $\begin{array}{l}\text { PLT turnover, MPV } \\
\uparrow\end{array}$ & LTA $\uparrow$ & $\begin{array}{l}108 \\
109 \\
131- \\
133 \\
\end{array}$ \\
\hline $\begin{array}{l}\text { Myelopro- } \\
\text { liferative } \\
\text { neoplasms (ET, } \\
\text { PCV) }\end{array}$ & $\begin{array}{l}\text { prothrom- } \\
\text { botic }\end{array}$ & hyper-reactive & PLT count $\uparrow$ & $\begin{array}{l}\text { LTA, CD62P, CAT } \\
\uparrow\end{array}$ & $\begin{array}{l}110 \\
111\end{array}$ \\
\hline \multicolumn{6}{|c|}{ Positively primed and secondarily inactive (low responsive) } \\
\hline $\begin{array}{l}\text { Cancers (solid } \\
\text { tumors) }\end{array}$ & $\begin{array}{l}\text { prothrom- } \\
\text { botic }\end{array}$ & $\begin{array}{l}\text { hyper-reactive } \\
\text { (exhausted) }\end{array}$ & $\begin{array}{l}\text { SCD62P, PEV } \uparrow \\
\text { PLT count } \uparrow\end{array}$ & $\begin{array}{l}\text { CD62P, CD63, } \\
\text { GPIb } \uparrow \\
\text { TxB2 } \downarrow\end{array}$ & $\begin{array}{l}112- \\
115\end{array}$ \\
\hline Sepsis & $\begin{array}{l}\text { thrombosis, } \\
\text { bleeding }\end{array}$ & $\begin{array}{l}\text { hyper-reac- } \\
\text { tive/ } \\
\text { exhausted }\end{array}$ & $\begin{array}{l}\text { PLT count } \downarrow \\
\text { sCD62P, sTLT, PEV } \uparrow\end{array}$ & $\begin{array}{l}\text { CD62P } \uparrow \\
\text { LTA, fibrinogen } \\
\text { binding } \downarrow \\
\text { (severe sepsis) } \\
\end{array}$ & $\begin{array}{l}116 \\
119\end{array}$ \\
\hline Stroke & bleeding & exhausted & $\begin{array}{l}\text { sCD62P, } \beta T G ~ \uparrow \\
\text { TxB2 (urine) } \uparrow\end{array}$ & $\begin{array}{l}\text { CD62P, CD63 } \downarrow \\
\text { mepacrine } \downarrow\end{array}$ & $\begin{array}{l}120- \\
122\end{array}$ \\
\hline $\begin{array}{l}\text { Major trauma } \\
\text { (brain) }\end{array}$ & bleeding & $\begin{array}{l}\text { hypo-respon- } \\
\text { sive }\end{array}$ & $\begin{array}{l}\text { PEV } \\
\text { CD62P, PAC mAb } \\
\text { (unstimulated) }\end{array}$ & LTA $\downarrow$ & $\begin{array}{l}123 \\
124\end{array}$ \\
\hline $\begin{array}{l}\text { Viral hemor- } \\
\text { rhagic fever }\end{array}$ & $\begin{array}{l}\text { thrombosis } \\
\text { (bleeding) }\end{array}$ & $\begin{array}{l}\text { exhausted/ } \\
\text { reticulated }\end{array}$ & $\begin{array}{l}\text { sCD62P, sGPVI } \uparrow \\
\text { TPO, IPF, MPV 个 }\end{array}$ & $\begin{array}{l}\alpha \mathrm{llb} \beta 3, \mathrm{CD} 62 \mathrm{P}, \\
\mathrm{CD63} \downarrow\end{array}$ & $\begin{array}{l}125 \\
126\end{array}$ \\
\hline
\end{tabular}

Abbreviations: CAT, calibrated automated thrombin generation in PRP; ET, essential thrombocytopenia; IPF, immature platelet fraction; LTA, light transmission aggregometry; MPV, mean platelet volume; NO, 
nitric oxide; PCV, polycythemia vera; PEV, platelet-derived extracellular vesicles; PF4, platelet factor 4; PLT, platelet; PS, phosphatidylserine; s, soluble; $\beta$-TG, $\beta$-thromboglobulin; s, soluble; TLT, TREM-like transcript-1; TPO, thrombopoietin.

papers refer to either a gain-of-function (high-responsive platelets) or a loss-of-function (low-responsive platelets). A still unresolved question is how these functional changes relate to the formation of specific platelet populations.

\subsection{Negatively primed platelets in disease}

The genetic makeup of a subject co-determines platelet responsiveness. Rare genetic modifications of over 25 different platelet proteins have been described that result in low-responsive platelets, and associate with bleeding disorders. ${ }^{98,}{ }^{99}$ Loss-offunction mutations, often accompanied by thrombocytopenia, in particular concern genes of transcription factors, the actin-myosin and tubulin cytoskeletons, signaling proteins, and granule secretion-regulating proteins. ${ }^{98,99}$ Genetic polymorphisms in these and other genes may also influence platelet responsiveness.

A clear example how a genetic deficiency can imbalance the formation of platelet populations is the Scott syndrome, a mild bleeding disorder. Herein, mutations in the ANO6 gene result in the near absence of the procoagulant, phosphatidylserine-exposing platelet population due to inability of phospholipid scrambling, ${ }^{21,22}$ resulting in a severely compromised fibrin formation. ${ }^{68}$

In addition to genetics, there is accumulating evidence for acquired forms of lowresponsive platelets. A primary loss-of-function is observed in patients with hematological malignancies and thrombocytopenia. For instance, platelets from patients with acute leukemia can be impaired in adhesion, aggregation and secretion. These functional defects are likely a consequence of dysfunctional megakaryocytopoiesis. ${ }^{100,} 101$

In patients with renal failure and associated uremia, it is considered that impaired platelet adhesion to the vessel wall is the main cause of bleeding. Several indicators of low platelet responsiveness are described, including impaired $\mathrm{Ca}^{2+}$ fluxes, reduced secretion and low platelet aggregation. ${ }^{102}$ On the other hand, also impaired platelet adhesion to the vessel wall due to anemia may explain the hemorrhagic complications in these patients. A low hematocrit limits the margination of platelets towards the vessel wall, and might furthermore restrict the availability of red blood cell-derived ADP and the scavenging effect of hemoglobin on nitric oxide as a platelet inhibitor. ${ }^{102}$ Other papers report that renal failure increases the risk of thrombosis, e.g. by increasing the population of apoptotic, phosphatidylserine-exposing platelets. ${ }^{103}$

\subsection{Positively primed platelets in disease}

Diabetes has often been associated with platelet hyperreactivity, i.e. enhanced platelet adhesion, activation, aggregation and platelet-dependent thrombin generation. ${ }^{104}$ This increased responsiveness of platelets is explained by high bloodglucose levels, oxidative stress and increased vascular shear stress. ${ }^{105}$ Under these hyperglycemic conditions, advanced glycation end products (AGEs) are generated, which 
activate platelets via the CD36 pathway. ${ }^{106}$ Further, activation of the receptor of AGEs (RAGE) by S100A8/A9 may lead to enhanced production of TPO, thereby increasing platelet production from megakaryocytes upon hyperglycemia. ${ }^{107}$ In diabetic patients, antiplatelet treatment strategies were shown to be effective for the secondary prevention of ischemic complications. ${ }^{104}$

Plasma cholesterol levels can positively prime platelets, such as manifested by the enhanced activity and larger size of platelets from patients with familial hypercholesterolemia, in whom total lipid and low-density lipoprotein levels are severely elevated. Accumulation of cholesterol in the platelet membrane enhances platelet aggregation by influencing the membrane structure and signaling via surface receptors. ${ }^{108,}$ ${ }^{109}$ Hypercholesterolemia is also linked to increased platelet production and turnover. ${ }^{108}$ Similarly, oxidation of low-density lipoproteins in the intima of atherosclerotic lesions is considered to result in enhanced platelet activation. ${ }^{108}$

In the myeloproliferative neoplasms, essential thrombocytosis and polycythemia vera, quantitative as well as qualitative platelet abnormalities have been identified. Particularly in patients carrying the JAK2 ${ }^{\mathrm{V} 617 F}$ mutation, for whom aspirin treatment is recommended, platelet responsiveness is increased (secretion, aggregation and procoagulant activity), which effect is in part associated with thromboembolic complications. ${ }^{110,111}$

Table 2. Markers of the different platelet populations. For abbreviations and explanation, see text and Table 1.

\begin{tabular}{|l|l|}
\hline Platelet population & Markers \\
\hline Reticulated & mRNA (immature platelet fraction) \\
\hline Secretory & $\begin{array}{l}\text { PF4, } \beta \text { TG release } \\
\text { CD62P, CD63 expression } \\
\text { TxB formation, serotonin }\end{array}$ \\
\hline Aggregating & $\begin{array}{l}\text { fibrinogen binding } \\
\text { PAC1 mAb binding (activated } \alpha_{\text {ubr }} \beta_{3} \text { integrin) } \\
\text { platelet-leukocyte aggregate formation }\end{array}$ \\
\hline Procoagulant & $\begin{array}{l}\text { PS exposure, coagulation factor binding } \\
\text { extracellular vesicle formation }\end{array}$ \\
\hline Coated & $\begin{array}{l}\text { PS exposure, } \\
\text { fibrin binding, transglutaminase activity } \\
\text { extracellular vesicle formation }\end{array}$ \\
\hline Apoptotic & $\begin{array}{l}\text { PS exposure, } \\
\text { extracellular vesicle formation }\end{array}$ \\
\hline
\end{tabular}




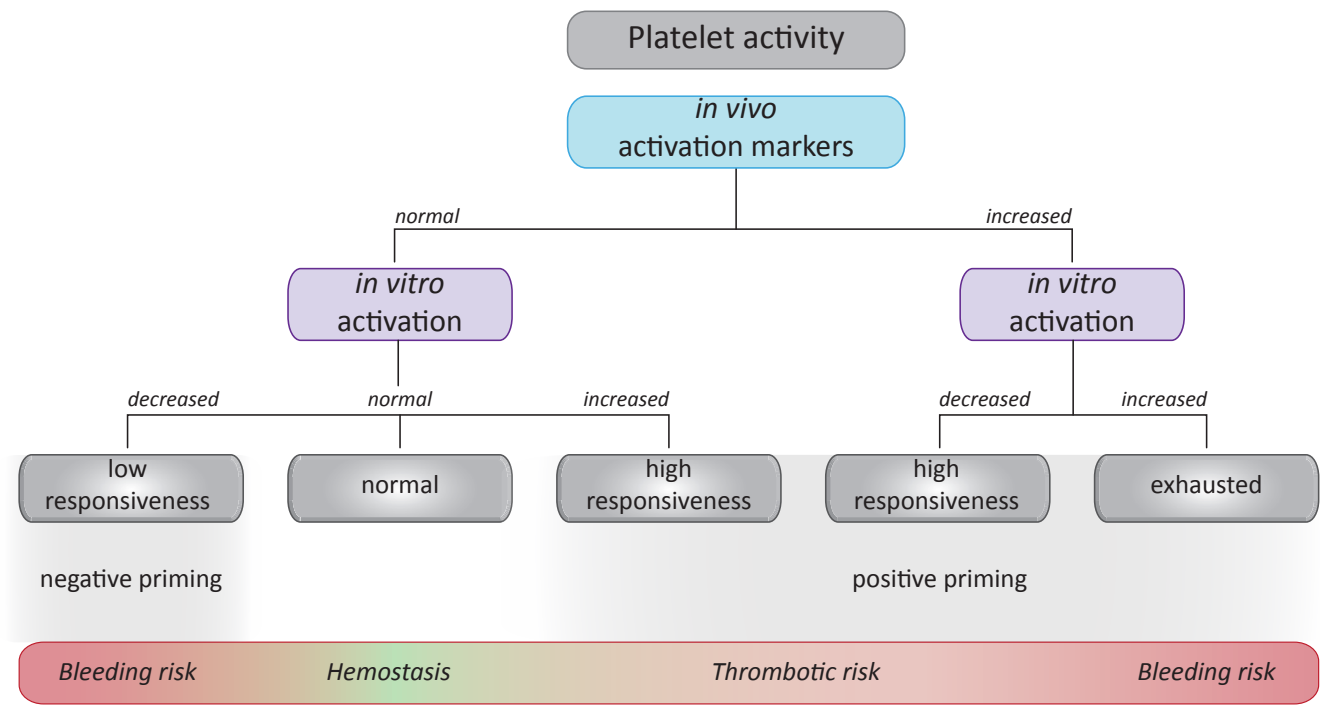

Figure 4. Concept of negative and positive platelet priming resulting in changed platelet activation markers in vivo and altered platelet activities in vitro. Flow chart for assessing changes in platelet functions in patients with thrombosis or bleeding risk.

\subsection{Positively primed and secondarily inactivated platelets in disease}

Despite the presence of positive priming molecules in vivo, platelet activation in vitro may appear to be impaired. This can be a direct consequence of prior activation in the circulation resulting in secondary loss of function. In such cases, authors describe the dysfunctional platelets often as exhausted platelets.

In patients with different types of solid tumors, apart from a high platelet count, platelet activation can be enhanced in vivo, as deduced from the presence of plateletderived soluble (s)CD62P in the blood plasma. ${ }^{112}$ This associates in vitro with a low-level of platelet activation, leading to the assumption that these platelets are 'exhausted', i.e. displaying a secondarily down-regulated responsiveness to agonists (CD62P, CD63 expression), although the primarily enhanced activation may predispose patients to an increased risk of thromboembolic complications. ${ }^{113,114}$ Another marker of ongoing platelet activation in cancer patients is the accumulation of platelet-derived extracellular vesicles, interestingly, with almost no effect on mean platelet volume. ${ }^{115}$ Strikingly, in those patients a high mean platelet volume associates (though with low odds ratio) with a decreased risk of venous thromboembolism. ${ }^{113}$

In sepsis, as a life-threatening condition of systemic inflammation, platelets are positively primed as a result of endothelial damage or activation in combination with enhanced coagulant activity. This leads to increased plasma levels of platelet secretion products as well as platelet-derived extracellular vesicles. ${ }^{116} \mathrm{It}$ has been demonstrated that several pathogens can interact with platelets via Toll-like receptors, FcyRIla and/ 
or integrin $\alpha_{116} \beta_{3^{\prime}}$, thus inducing platelet activation. ${ }^{116-118}$ In severe sepsis, also secondary defects in platelet function have been observed, often concomitantly with a lowering in platelet count. ${ }^{116,119}$

Papers studying patients who suffered a stroke, report increased levels in the circulation of platelet activation markers, including SCD62P, $\beta$-thromboglobulin and thromboxane $B_{2}$, in those patients with a high risk of bleeding. ${ }^{120,}{ }^{121}$ Since this is accompanied by an impaired platelet activation tendency in vitro, the authors type such platelets as exhausted. ${ }^{122} \mathrm{~A}$ similar platelet phenotype is expected in patients with acute coronary syndrome before the initiation of antiplatelet therapy. An exhausted platelet type has also been observed after major trauma, in particular brain injury, where plasma markers of platelet activation are elevated, while the platelets after isolation are hypo-responsive in terms of aggregation. ${ }^{123,124}$ The suggestion was made that subendothelial tissue exposure upon injury causes a low level of platelet activation, resulting in dysfunctional circulating platelets with an activation history.

Higher fractions (populations) of reticulated platelets have been detected in patients with viral hemorrhagic fever due to hantaviral disease, which was accompanied by increased levels of activation markers such as SCD62P and SGPVI, followed by a secondary loss in platelet activity in vitro. ${ }^{125} \mathrm{~A}$ similar phenotype of exhausted platelets has been described for patients with a severe dengue infection. ${ }^{126}$

It is currently unclear how the different pathologies induce specific changes in platelet function. The pathological environment may alter one specific platelet population or it rather affects all circulating platelets and thereby elicits a shift in the distribution of the different platelet populations. It can be reasonably presumed that this change is specific for the pathological environment. Furthermore, the impact of a pathological condition on platelet function may be multifactorial (e.g. diabetes) and therefore even more complex.

\section{Platelet heterogeneity and risk assessment for bleeding and thrombosis: recommendations}

For diagnosing a known or suspected risk of thrombosis or bleeding, current routine measurements include (i) platelet count and mean volume, (ii) plasma markers of in vivo platelet activation, and (iii) in vitro platelet activation tests. Particularly, the latter tests are used for the monitoring of anti-platelet therapy. Table 2 presents an outline of how different test outcomes relate to distinct platelet populations, provided that sample processing is up to standards.

The proposed concept of partly overlapping platelet populations that are subjected to positive/negative priming can shed new light on the often paradoxical information obtained from different diagnostic platelet function and activity tests. Figure 4 shows a flow chart aiming to structure the information obtained from such tests for a risk estimate of hemorrhage or thrombosis. From the figure, it will be clear that information on both activation markers in vivo and platelet responsiveness in vitro is needed to obtain a complete picture. It is noted though that platelets are deprived of endothelial inhibitors when performing in vitro measurements, which influences their 
responsiveness. Assay measurements should preferably include markers of different platelet populations, given their specific contribution to hemostasis or thrombosis. Understanding of the redistribution of platelet populations in patients with particular hemorrhagic or thrombotic tendencies will also aid in designing an optimized treatment. One such an example is the administration of DDAVP to patients with a mild congenital platelet function defect which selectively augments the formation of coated platelets by enhancing $\mathrm{Na}^{+} / \mathrm{Ca}^{2+}$ mobilization. ${ }^{127}$

\section{Practice Points}

- Platelet heterogeneity can originate from differences between megakaryocytes, variation in proplatelet formation, unequal platelet priming and environmental conditions, as well as platelet ageing. Changes in the physiological environment upon disease can hence influence platelet populations and platelet responses.

- Combining of both in vivo and in vitro markers of platelet activation and activity permits better assessment of a platelet-related thrombotic or hemorrhage risk.

\section{Research agenda}

- How the pathophysiological environment affects platelet populations and their responsiveness is incompletely understood and warrants further investigation.

- The causes and consequences of platelet heterogeneity have only partly been resolved. Characterization of the preferential response spectrum of certain platelet populations may contribute to risk factor assessments for prediction of thrombotic or hemorrhagic complications.

\section{Funding}

PvdM was supported by an EHA-ISTH Research Fellowship award granted by the European Hematology Association and the International Society of Thrombosis and Haemostasis (2014).

\section{Conflict of interest}

The authors report no conflicts of interest. 


\section{References}

1. Long MW. Population heterogeneity among cells of the megakaryocytic lineage. Stem Cells. 1993;11(1):3340.

2. Paulus JM, Levin J, Debili N, Albert A, Vainchenker W. Genesis of clone size heterogeneity in megakaryocytic and other hemopoietic colonies: The stochastic model revisited. Exp Hematol. 2001;29(11):1256-1269.

3. den Dekker E, van Abel M, van der Vuurst H, van Eys GJJM, Akkerman JWN, Heemskerk JW. Cell-to-cell variability in the differentiation program of human megakaryocytes. Biochim Biophys Acta. 2003;1643(13):85-94.

4. Moreau T, Evans AL, Vasquez L, Tijssen MR, Yan Y, Trotter MW, et al. Large-scale production of megakaryocytes from human pluripotent stem cells by chemically defined forward programming. Nat Commun. 2016;7:11208.

5. Rondina MT, Weyrich AS. Regulation of the genetic code in megakaryocytes and platelets. J Thromb Haemost. 2015;13(Suppl 1):S26-S32.

6. Chaabane C, Dally S, Corvazier E, Bredoux R, Bobe R, Ftouhi B, et al. Platelet PMCA- and SERCA-type Ca ${ }^{2+}$ ATPase expression in diabetes: a novel signature of abnormal megakaryocytopoiesis. J Thromb Haemost. 2007;5(10):2127-2135.

7. Lood C, Amisten S, Gullstrand B, Jönsen A, Allhorn M, Truedsson L, et al. Platelet trancriptional profile and protein expression in patients with systemic lupus erythematosus: up-regulation of the type I interferon system is strongly associated with vascular disease. Blood. 2010;116(11):1951-1957.

8. Machlus KR, Italiano Jr JE. The incredible journey: From megakaryocyte development to platelet formation. J Cell Biol. 2013;201(6):785-796.

9. Jackson CW, Jennings LK. Heterogeneity of fibrinogen receptor expression on platelets activated in normal plasma with ADP: analysis by flow cytometry. Br J Haematol. 1989;72(3):407-414.

10. Munnix ICA, Cosemans JMEM, Auger JM, Heemskerk JWM. Platelet response heterogeneity in thrombus formation. Thromb Haemost. 2009;102(6):1149-1156.

11. Heemskerk JW, Willems GM, Rook MB, Sage SO. Ragged spiking of free calcium in ADP-stimulated human platelets: regulation of puff-like calcium signals in vitro and ex vivo. J Physiol. 2001;15(535):625-635.

12. Eckly A, Rinckel JY, Proamer F, Ulas N, Joshi S, Whiteheart SW, et al. Respective contributions of single and compound granule fusion to secretion by activated platelets. Blood. 2016;128(21):2538-2549.

13. Ollivier V, Syvannarath V, Gros A, Butt A, Loyau S, Jandrot-Perrus M, et al. Collagen can selectively trigger a platelet secretory phenotype via glycoprotein VI. Plos One. 2014;9(8):e104712.

14. van Geffen JP, Kleinegris MC, Verdoold R, Baaten CC, Cosemans JM, Clemetson KJ, et al. Normal platelet activation profile in patients with peripheral arterial disease on aspirin. Thromb Res. 2015;135(3):513-520.

15. Mattheij NJA, Gilio K, Van Kruchten R, Jobe SM, Wieschhaus AJ, Chishti AH, et al. Dual mechanism of integrin $\alpha$ llb $\beta 3$ closure in procoagulant platelets. J Biol Chem. 2013;288(19):13325-13336.

16. Versteeg HH, Heemskerk JWM, Levi M, Reitsma PS. New fundamentals in hemostasis. Physiol Rev. 2013;93(1):327-358.

17. Mattheij NJ, Swieringa F, Mastenbroek TG, Berny-Lang MA, May F, Baaten CC, et al. Coated platelets function in platelet-dependent fibrin formation via integrin $\alpha$ llb $\beta 3$ and transglutaminase factor XIII. Haematologica. 2016;101(4):427-436.

18. Dale GL. Coated-platelets: an emerging component of the procoagulant response. J Thromb Haemost. 2005;3(10):2185-2192.

19. Heemskerk JWM, Mattheij N, Cosemans JMEM. Platelet-based coagulation: different populations, different functions. J Thromb Haemost. 2013;11(1):2-11.

20. Podoplelova NA, Sveshnikova AN, Kotova YN, Eckly A, Receveur N, Nechipurenko DY, et al. Coagulation factors bound to procoagulant platelets concentrate in cap structures to promote clotting. Blood. 2016;128(13):1745-1755.

21. Solari F, Mattheij NJ, Burkhart JM, Swieringa F, Collins P, Cosemans JM, et al. Combined quantification of the global proteome, phosphoproteome, and proteolytic cleavage to characterize altered platelet functions in the human Scott syndrome. Mol Cell Proteomics. 2016;15(10):3154-69.

22. Mattheij NJA, Braun A, van Kruchten R, Castoldi E, Pircher J, Baaten CCFMJ, et al. Survival protein anoctamin-6 controls multiple platelet responses including phospholipid scrambling, swelling and protein cleavage. FASEB J. 2016;30(2):727-37. 
23. Van Kruchten R, Mattheij NJ, Saunders C, Feijge MA, Swieringa F, Wolfs JL, et al. Both TMEM16Fdependent and TMEM16F-independent pathways contribute to phosphatidylserine exposure in platelet apoptosis and platelet activation. Blood. 2013;121(10):1850-1857.

24. Thompson CB, Eaton KA, Princiotta SM, Rushin CA, Valeri CR. Size dependent platelet subpopulations: relationship of platelet volume to ultrastructure, enzymatic activity, and function. $\mathrm{Br} \mathrm{J}$ Haematol. 1982;50(3):509-519.

25. Jakubowski JA, Thompson CB, Vaillancourt R, Valeri CR, Deykin D. Arachidonic acid metabolism by platelets of differing size. Br J Haematol. 1983;53(3):503-11.

26. Robinson MSC, Machin SJ, Mackie IJ, Harrison P. In vivo biotinylation studies: specificity of labelling of reticulated platelets by thiazole orange and mepacrine. Br J Haematol. 2000;108(4):859-864.

27. McBane II RD, Gonzalez C, Hodge DO, Wysokinski WE. Propensity for young reticulated platelet recruitment into arterial thrombi. J Thromb Thrombolysis. 2014;37(2):148-154.

28. Hoffmann JJ, van den Broek NM, Curvers J. Reference intervals of reticulated platelets and other platelet parameters and their associations. Arch Pathol Lab Med. 2013;137(11):1635-1640.

29. Hoffmann JJ. Reticulated platelets: analytical aspects and clinical utility. Clin Chem Lab Med. 2014;52(8):1107-1117.

30. Robinson MSC, Mackie IJ, Machin SJ, Harrison P. Two colour analysis of reticulated platelets. Clin Lab Haem. 2000;22(4):211-213.

31. Harrison P, Goodall AH. "Message in the Platelet"- more than just vestigial mRNA! Platelets. 2008;19(6):395-404.

32. Briggs C, Kunka S, Hart D, Oguni S, Machin SJ. Assessment of an immature platelet fraction (IPF) in peripheral thrombocytopenia. Br J Haematol. 2004;126(1):93-99.

33. Alhasan AA, Izuogu OG, Al-Balool HH, Steyn JS, Evans A, Colzani M, et al. Circular RNA enrichment in platelets is a signature of transcriptome degradation. Blood. 2016;127(9):e1-e11.

34. Simon LM, Edelstein LC, Nagalla S, Woodley AB, Chen ES, Kong X, et al. Human platelet microRNA-mRNA networks associated with age and gender revealed by integrated plateletomics. Blood. 2014;123(16):e37-e45. 35. Pujol-Moix N, Vazquez-Santiago M, Morera A, Ziyatdinov A, Remacha A, Nomdedeu JF, et al. Genetic determinants of platelet-large-cell ratio, immature platelet fraction, and other platelet-related phenotypes. Thromb Res. 2015;136(2):361-366.

36. McManus DD, Freedman JE. MicroRNAs in platelet function and cardiovascular disease. Nat Rev Cardiol. 2015;12(12):711-717.

37. Lindsay CR, Edelstein LC. MicroRNAs in platelet physiology and function. Semin Thromb Hemost. 2016;42(3):215-222.

38. Li R, Hoffmeister KM, Falet H. Glycans and the platelet life cycle. Platelets. 2016;27(6):505-511.

39. Lebois M, Josefsson EC. Regulation of platelet lifespan by apoptosis. Platelets. 2016;27(6):497-504.

40. Josefsson EC, James C, Henley KJ, Debrincat MA, Rogers KL, Dowling MR, et al. Megakaryocytes possess a functional intrinsic apoptosis pathway that must be restrained to survive and produce platelets. J Exp Med. 2011;208(10):2017-2031.

41. Pleines I, Lebois M, Au AEL, Lane RM, Henley KJ, Corbin J, et al. Extended platelet in vivo survival results in exhausted platelets. Blood. 2015;126(23):416.

42. Jackson SP, Schoenwaelder SM. Procoagulant platelets: are they necrotic? Blood. 2010;116(12):20112018.

43. Vogler M, Hamali HA, Sun XM, Bampton ETW, Dinsdale D, Snowden RT, et al. BCL2/BCL-X inhibition induces apoptosis, disrupts cellular calcium homeostasis, and prevents platelet activation. Blood. 2011;117(26):7145-7154.

44. Grozovsky R, Begonja AJ, Liu K, Visner G, Hartwig JH, Falet H, et al. The Ashwell-Morell receptor regulates hepatic thrombopoietin production via JAK-STAT3 signaling. Nat Med. 2015;21(1):47-54.

45. Bevers EM, Comfurius P, van Rijn JL, Hemker HC, Zwaal RF. Generation of prothrombin-converting activity and the exposure of phosphatidylserine at the outer surface of platelets. Eur J Biochem. 1982;122(2):429436.

46. Swieringa F, Kuijpers MJE, Lamers MME, Van der Meijden PE, Heemskerk JW. Rate-limiting roles of the tenase complex of factors VIII and IX in platelet procoagulant activity and formation of platelet-fibrin thrombi under flow. Haematologica. 2015;100(6):748-756.

47. Nieswandt B, Brakebusch C, Bergmeier W, Schulte V, Bouvard D, Mokhtari-Nejad R, et al. Glycoprotein VI but not $\alpha 2 \beta 1$ integrin is essential for platelet interaction with collagen. EMBO J. 2001;20(9):2120-2130. 
48. Nieswandt B, Watson SP. Platelet-collagen interaction: is GPVI the central receptor? Blood. 2003;102(2):449-461.

49. Gibbins JM. Platelet adhesion signalling and the regulation of thrombus formation. J Cell Sci. 2004;117(Pt 16):3415-3425.

50. Siljander PRM, Farndale RW, Feijge MAH, Comfurius P, Kos S, Bevers EM, et al. Platelet adhesion enhances the glycoprotein VI-dependent procoagulant response: Involvement of p38 MAP Kinase and calpain. Arterioscler Thromb Vasc Biol. 2001;21(4):618-627.

51. Heemskerk JW, Kuijpers MJ, Munnix IC, Siljander PRM. Platelet collagen receptor and coagulation. A characteristic platelet response as possible target for antithrombotic treatment. Trends Cardiovasc Med. 2005;15(3):86-92.

52. Agbani EO, van den Bosch MT, Brown E, Williams CM, Mattheij NJ, Cosemans JM, et al. Coordinated membrane ballooning and procoagulant spreading in human platelets. Circulation. 2015;132(15):1414-1424.

53. Savage B, Almus-Jacobs F, Ruggeri ZM. Specific synergy of multiple substrate-receptor interactions in platelet thrombus formation under flow. Cell. 1998;94(5):657-666.

54. Siljander PRM, Munnix ICA, Smethurst PA, Deckmyn H, Lindhout T, Ouwehand WH, et al. Platelet receptor interplay regulates collagen-induced thrombus formation in flowing human blood. Blood. 2004;103(4):13331341.

55. Auger JM, Kuijpers MJE, Senis YA, Watson SP, Heemskerk JW. Adhesion of human and mouse platelets to collagen under shear: a unifying model. FASEB J. 2005;19(7):825-827.

56. Gilio K, Van Kruchten R, Braun A, Berna-Erro A, Feijge MAH, Stegner D, et al. Roles of platelet STIM1 and Orai1 in glycoprotein VI- and thrombin-dependent procoagulant activity and thrombus formation. J Biol Chem. 2010;285(31):23629-23638.

57. Munnix ICA, Kuijpers MJE, Auger JM, Thomassen CMLGD, Panizzi P, van Zandvoort MAMJ, et al. Segregation of platelet aggregatory and procoagulant microdomains in thrombus formation. Regulation by transient integrin activation. Arterioscler Thromb Vasc Biol. 2007;27(11):2484-2490.

58. Jackson SP. Arterial thrombosis: insidious, unpredictable and deadly. Nat Med. 2011;17(11):1423-1436.

59. de Witt SM, Verdoold R, Cosemans JM, Heemskerk JW. Insights into platelet-based control of coagulation. Thromb Res. 2014;133(Suppl 2):S139-S148.

60. Vaiyapuri S, Jones Cl, Sasikumar P, Moreas LA, Munger SJ, Wright JR, et al. Gap junctions and connexins hemichannels underpin hemostasis and thrombosis. Circulation. 2012;125(20):2479-2491.

61. Nesbitt WS, Giuliano S, Kulkarni S, Dopheide SM, Harper IS, Jackson SP. Intercellular calcium communication regulates platelet aggregation and thrombus growth. J Cell Biol. 2003;160(7):1151-1161.

62. de Witt SM, Swieringa F, Cavill R, Lamers MME, van Kruchten R, Mastenbroek T, et al. Identification of platelet function defects by multi-parameter assessment of thrombus formation. Nat Commun. 2014;5:4257. 63. Stalker TJ, Welsh JD, Brass LF. Shaping the platelet response to vascular injury. Curr Opin Hematol. 2014;21(5):410-417.

64. Welsh JD, Poventud-Fuentes I, Sampietro S, Diamond SL, Stalker TJ, Brass LF. Hierarchical organization of the hemostatic response to penetrating injuries in the mouse macrovasculature. J Thromb Haemost. 2017;15(3):526-537.

65. Westein E, van der Meer AD, Kuijpers MJE, Frimat JP, van den Berg A, Heemskerk JW. Atherosclerotic geometries exacerbate pathological thrombus formation poststenosis in a von Willebrand factor-dependent manner. PNAS. 2013;110(4):1357-1362.

66. Ivanciu L, Krishnaswamy S, Camire RM. New insights into the spatiotemporal localization of prothrombinase in vivo. Blood. 2014;124(11):1705-1714.

67. Cosemans JM, Schols SEM, Stefanini L, de Witt SM, Feijge MAH, Hamulyák K, et al. Key role of glycoprotein $\mathrm{Ib} / \mathrm{V} / \mathrm{IX}$ and von Willebrand factor in platelet activation-dependent fibrin formation at low shear flow. Blood. 2011;117(2):651-660.

68. Swieringa F, Baaten CC, Mastenbroek T, Rijnveld N, van der Laan K, Collins P, et al. Platelet-dependent control of fibrin distribution and micro-elasticity in thrombus formation under flow. Arterioscler Thromb Vasc Biol. 2016;36(4):692-699.

69. Verhoef JJ, Barendrecht AD, Nickel KF, Dijkxhoorn K, Kenne E, Labberton L, et al. Polyphosphate nanoparticles on the platelet surface trigger contact system activation. Blood. 2017;129(12):1707-1717.

70. Whyte CS, Swieringa F, Mastenbroek T, Lionikiene AS, Lancé MD, van der Meijden PE, et al. Plasminogen associates with phosphatidylserine-exposing platelets and contributes to thrombus lysis under flow. Blood. 2015;125(16):2568-2578. 
71. Henson R, Shallice T, Dolan R. Neuroimaging evidence for dissociable forms of repetition priming. Science. 2000;287(5456):1269-1272.

72. Keularts IMLW, van Gorp RMA, Feijge MAH, Vuist MWJ, Heemskerk JWM. $\alpha_{2 A}$-Adrenergic receptor stimulation potentiates calcium release in platelets by modulating cAMP levels. J Biol Chem. 2000;275(3):17631772.

73. Montrucchio G, Brizzi MF, Calosso G, Marengo S, Pegoraro L, Camussi G. Effects of recombinant human megakaryocyte growth and development factor on platelet activation. Blood. 1996;87(7):2762-2768.

74. Pasquet JM, Gross BS, Gratacap MP, Quek L, Pasquet S, Payrastre B, et al. Thrombopoietin potentiates collagen receptor signaling in platelets through a phosphatidylinositol 3-kinase-dependent pathway. Blood. 2000;95(11):3429-3434.

75. Oda A, Miyakawa Y, Druker BJ, Ozaki K, Yabusaki K, Shirasawa Y, et al. Thrombopoietin primes human platelet aggregation induced by shear stress and by multiple agonists. Blood. 1996;87(11):4664-4670.

76. Hers I. Insulin-like growth factor-1 potentiates platelet activation via the IRS/PI3Ka pathway. Blood. 2007;110(13):4243-4252.

77. Blair TA, Moore SF, Hers I. Circulating primers enhance platelet function and induce resistance to antiplatelet therapy. J Thromb Haemost. 2015;13(8):1479-1493.

78. Cosemans JM, van Kruchten R, Olieslagers S, Schurgers LJ, Verheyen FK, Munnix IC, et al. Potentiating role of Gas6 and Tyro3, Axl and Mer (TAM) receptors in human and murine platelet activation and thrombus stabilization. J Thromb Haemost. 2010;8(8):1797-1808.

79. Kuijpers MJ, Mattheij NJ, Cipolla L, van Geffen JP, Lawrence T, Donners MM, et al. Platelet CD40L modulates thrombus growth via phosphatidylinositol 3-kinase $\beta$, and not via CD40 and IkB kinase $\alpha$. Arterioscler Thromb Vasc Biol. 2015;35(6):1374-1381.

80. Pircher J, Merkle M, Wörnle M, Ribeiro A, Czermak T, Stampnik Y, et al. Prothrombotic effects of tumor necrosis factor alpha in vivo are amplified by the absence of TNF-alpha receptor subtype 1 and require TNFalpha receptor subtype 2. Arthritis Res Ther. 2012;14(5):R225.

81. Pignatelli P, De Biase L, Lenti L, Tocci G, Brunelli A, Cangemi R, et al. Tumor necrosis factor-alpha as trigger of platelet activation in patients with heart failure. Blood. 2005;106(6):1992-1994.

82. Nesbitt WS, Westein E, Tovar-Lopez FJ, Tolouei E, Mitchell A, Fu J, et al. A shear gradient-dependent platelet aggregation mechanism drives thrombus formation. Nat Med. 2009;15(6):665-673.

83. Zhu W, Gregory JC, Org E, Buffa JA, Gupta N, Wang Z, et al. Gut microbial metabolite TMAO enhances platelet hyperreactivity and thrombosis risk. Cell. 2016;165(1):111-124.

84. Weyrich AS, Zimmerman GA. Platelets in lung biology. Annu Rev Physiol. 2013;75:569-591.

85. Aster RH. Pooling of platelets in the spleen: role in the pathogenesis of "hypersplenic" thrombocytopenia. J Clin Invest. 1966;45(5):645-657.

86. Lefrançais E, Ortiz-Muñoz G, Caudrillier A, Mallavia B, Liu F, Sayah DM, et al. The lung is a site of platelet biogenesis and a reservoir for haematopoietic progenitors. Nature. 2017;544(7648):105-109.

87. Rex S, Freedman JE. Platelets: Chapter 13: Inhibition of platelet function by the endothelium. Michelson AD, editor: Academic press; 2006; 2: 251-279.

88. Swieringa F, Kuijpers MJE, Heemskerk JW, Van der Meijden PE. Targeting platelet receptor function in thrombus formation: The risk of bleeding. Blood Rev. 2014;28(1):9-21.

89. Mawhin MA, Tilly P, Fabre JE. The receptor EP3 to PGE2: a rational target to prevent atherosthrombosis without inducing bleeding. Prostaglandins Other Lipid Mediat. 2015;121(Pt A):4-16.

90. Naseem KM, Roberts W. Nitric oxide at a glance. Platelets. 2011;22(2):148-152.

91. Smolenski A. Novel roles of cAMP/cGMP-dependent signaling in platelets. J Thromb Haemost. 2012;10(2):167-176.

92. Beck F, Geiger J, Gambaryan S, Veit J, Vaudel M, Nollau P, et al. Time-resolved characterization of cAMP/ PKA-dependent signaling reveals that platelet inhibition is a concerted process involving multiple signaling pathways. Blood. 2014;123(5):e1-e10.

93. Trovati M, Mularoni EM, Burzacca S, Ponziani MC, Massucco P, Mattiello L, et al. Impaired insulin-induced platelet antiaggregating effect in obesity and in obese NIDDM patients. Diabetes. 1995;44(11):1318-1322.

94. Randriamboavonjy V, Fleming I. Insulin, insulin resistance, and platelet signaling in diabetes. Diabetes Care. 2009;32(4):528-530.

95. Senis YA. Protein-tyrosine phosphatases: a new frontier in platelet signal transduction. J Thromb Haemost. 2013;11(10):1800-1813.

96. Ruf W, Dorfleutner A, Riewald M. Specificity of coagulation. J Thromb Haemost. 2003;1(7):1495-1503. 
97. Phang M, Lazarus S, Wood LG, Garg M. Diet and thrombosis risk: nutrients for prevention of thrombotic disease. Semin Thromb Hemost. 2011;37(3):199-208.

98. Nurden AT, Nurden P. Inherited disorders of platelet function: selected updates. J Thromb Haemost. 2015;13(Suppl 1):S2-9.

99. Bianchi E, Norfo R, Pennucci V, Zini R, Manfredini R. Genomic landscape of megakaryopoiesis and platelet function defects. Blood. 2016;127(10):1249-1259.

100. Leinoe EB, Hoffmann MH, Kjaersgaard E, Johnsen HE. Multiple platelet defects identified by flow cytometry at diagnosis in acute myeloid leukaemia. Br J Haematol. 2004;127(1):76-84.

101. Foss B, Bruserud O. Platelet functions and clinical effects in acute myelogenous leukemia. Thromb Haemost. 2008;99(1):27-37

102. Mannucci PM, Tripodi A. Hemostatic defects in liver and renal dysfunction. Hematology Am Soc Hematol Educ Program. 2012;2012:168-173.

103. Lutz J, Menke J, Sollinger D, Schinzel H, Thürmel K. Haemostasis in chronic kidney disease. Nephrol Dial Transplant. 2014;29(1):29-40.

104. Rollini F, Franchi F, Muñiz-Lozano A, Angiolillo DJ. Platelet function profiles in patients with diabetes mellitus. J Cardiovasc Transl Res. 2013;6(3):329-345.

105. Arthur JF, Jandeleit-Dahm K, Andrews RK. Platelet hyperreactivity in diabetis: Focus on GPVI signaling - Are useful drugs already available? Diabetes. 2017;66(1):7-13.

106. Zhu W, Li W, Silverstein RL. Advanced glycation end products induce a prothrombotic phenotype in mice via interaction with platelet CD36. Blood. 2012;119(25):6136-6144.

107. Kraakman MJ, Lee MK, Al-Sharea A, Dragoljevic D, Barrett TJ, Montenont E, et al. Neutrophilderived S100 calcium-binding proteins A8/A9 promote reticulated thrombocytosis and atherogenesis in diabetes. J Clin Invest. 2017;127(6):2133-2147.

108. Wang N, Tall AR. Cholesterol in platelet biogenesis and activation. Blood. 2016;127(16):1943-1953.

109. Icli A, Aksoy F, Nar G, Kaymaz H, Alpay MF, Nar R, et al. Increased mean platelet volume in familial hypercholesterolemia. Angiology. 2016;67(2):146-150.

110. Moscardó A, Latorre A, Santos MT, Bonanad S, Vallés J. Platelet function in malignant hematological disorders. Curr Opin Oncol. 2015;27(6):522-531.

111. Panova-Noeva M, Marchetti M, Russo L, Tartari CJ, Leuzzi A, Finazzi G, et al. ADP-induced platelet aggregation and thrombin generation are increased in Essential Thrombocythemia and Polycythemia Vera. Thromb Res. 2013;132(1):88-93.

112. Riedl J, Pabinger I, Ay C. Platelets in cancer and thrombosis. Hamostaseologie. 2014;34(1):54-62.

113. Riedl J, Kaider A, Marosi C, Prager GW, Eichelberger B, Assinger A, et al. Decreased platelet reactivity in patients with cancer is associated with high risk of venous thromboembolism and poor prognosis. Thromb Haemost. 2017;117(1):90-98.

114. Boneu B, Bugat R, Boneu A, Eche N, Sie P, Combes PF. Exhausted platelets in patients with malignant solid tumors without evidence of active consumption coagulopathy. Eur J Cancer Clin Oncol. 1984;20(7):899903.

115. Falanga A, Tartari CJ, Marchetti M. Microparticles in tumor progression. Thromb Res. 2012;129(Suppl 1):S132-S136.

116. de Stoppelaar SF, van 't Veer C, van der Poll T. The role of platelets in sepsis. Thromb Haemost. 2014;112(4):666-677.

117. Berthet J, Damien P, Hamzeh-Cognasse H, Arthaud CA, Eyraud MA, Zéni F, et al. Human platelets can discriminate between various bacterial LPS isoforms via TLR4 signaling and differential cytokine secretion. Clin Immunol. 2012;145(3):189-200.

118. Fälker K, Klarström-Engström K, Bengtsson T, Lindahl TL, Grenegård $M$. The toll-like receptor 2/1 (TLR2/1) complex initiates human platelet activation via the src/Syk/LAT/PLC 22 signalling cascade. Cell Signal. 2014;26(2):279-286.

119. Lundahl TH, Petersson J, Fagerberg IH, Berg S, Lindahl TL. Impaired platelet function correlates with multi-organ dysfunction. A study of patients with sepsis. Platelets. 1998;9(3-4):223-225.

120. Iwamoto T, Kubo H, Takasaki M. Platelet activation in the cerebral circulation in different subtypes of ischemic stroke and Binswanger's disease. Stroke. 1995;26(1):52-56.

121. McConnell JP, Cheryk LA, Durocher A, Bruno A, Bang NU, Fleck JD, et al. Urinary 11-dehydrothromboxane B2 and coagulation activation markers measured within $24 \mathrm{~h}$ of human acute ischemic stroke. Neurosci Lett. 2001;313(1-2):88-92. 
122. Jurk K, Jahn U, van Aken H, Schriek C, Droste DW, Ritter MA, et al. Platelets in patients with acute ischemic stroke are exhausted and refractory to thrombin, due to cleavage of the seven-transmembrane thrombin receptor (PAR-1). Thromb Haemost 2004;91(2):334-344.

123. Jacoby RC, Owings JT, Holmes J, Battistella FD, Gosselin RC, Paglieroni TG. Platelet activation and function after trauma. J Trauma. 2001;51(4):639-647.

124. Kutcher ME, Redick BJ, McCreery RC, Crane IM, Greenberg MD, Cachola LM, et al. Characterization of platelet dysfunction after trauma. J Trauma Acute Care Surg. 2012;73(1):13-19.

125. Connolly-Andersen AM, Sundberg E, Ahlm C, Hultdin J, Baudin M, Larsson J, et al. Increased thrombopoiesis and platelet activation in Hantavirus-infected patients. J Infect Dis. 2015;212(7):1061-1069. 126. Michels M, Alisjahbana B, de Groot PG, Indrati AR, Fijnheer R, Puspita M, et al. Platelet function alterations in dengue are associated with plasma leakage. Thromb Haemost. 2014;112(2):352-362.

127. Colucci G, Stutz M, Rochat S, Conte T, Pavicic M, Reusser M, et al. The effect of desmopressin on platelet function: a selective enhancement of procoagulant COAT platelets in patients with primary platelet function defects. Blood. 2014;123(12):1905-1916.

128. Burkhart JM, Vaudel M, Gambaryan S, Radau S, Walter U, Martens L, et al. The first comprehensive and quantitative analysis of human platelet protein composition allows the comparative analysis of structural and functional pathways. Blood. 2012;120(15):e73-e82.

129. Israels SJ, McNicol A, Dean HJ, Cognasse F, Sellers EAC. Markers of platelet activation are increased in adolescents with type 2 diabetes. Diabetes Care. 2014;37(8):2400-2403.

130. Soma P, Swanepoel AC, du Plooy JN, Mqoco T, Pretorius E. Flow cytometric analysis of platelets in type 2 diabetes mellitus reveals 'angry' platelets. Cardiovasc Diabetol. 2016;15:52.

131. Calkin AC, Drew BG, Ono A, Duffy SJ, Gordon MV, Schoenwaelder SM, et al. Reconstituted highdensity lipoprotein attenuates platelet function in individuals with type 2 diabetes mellitus by promoting cholesterol efflux. Circulation. 2009;120(21):2095-2104.

132. Carvalho AC, Colman RW, Lees RS. Platelet function in hyperlipoproteinemia. N Engl J Med. 1974;290(8):434-438.

133. Pathansali R, Smith N, Bath P. Altered megakaryocyte-platelet haemostatic axis in hypercholesterolaemia. Platelets. 2001;12(5):292-297. 



\section{Chapter 3}

Gradual increase in thrombogenicity of juvenile platelets formed upon offset of prasugrel medication

Baaten CC, Veenstra LF, Wetzels RF, van Geffen JP, Swieringa F, de Witt SM, Henskens YM, Crijns H, Nylander S, van Giezen JJ, Heemskerk JW, van der Meijden PE

Haematologica 2015; 100 (9): 1131-1138

Reprinted with permission 


\section{Abstract}

In patients with acute coronary syndrome, dual antiplatelet therapy with aspirin and a $\mathrm{P}_{2} \mathrm{Y}_{12}$ inhibitor like prasugrel is prescribed for one year. Here, we investigated how the hemostatic function of platelets recovers after discontinuation of prasugrel treatment. Therefore, sixteen patients who suffered from ST-elevation myocardial infarction were investigated. Patients were treated with aspirin $(100 \mathrm{mg} / \mathrm{day}$, longterm) and stopped taking prasugrel $(10 \mathrm{mg} /$ day) after one year. Blood was collected at the last day of prasugrel intake and at 1, 2, 5, 12 and 30 days later. Platelet function in response to ADP was normalized between 5 and 30 days after treatment cessation and in vitro addition of the reversible $\mathrm{P}_{2} \mathrm{Y}_{12}$ receptor antagonist ticagrelor fully suppressed the regained activation response. Discontinuation of prasugrel resulted in the formation of an emerging subpopulation of ADP-responsive platelets, exhibiting high expression of active integrin $\alpha_{11 \mathrm{~b}} \beta_{3}$. Two different mRNA probes, thiazole orange and the novel 5'Cy5-oligo-dT probe revealed that this subpopulation consisted of juvenile platelets, which progressively contributed to platelet aggregation and thrombus formation under flow. During offset, juvenile platelets were overall more reactive than older platelets. Interestingly, the responsiveness of both juvenile and older platelets increased in time, pointing towards a residual inhibitory effect of prasugrel on the megakaryocyte level. In conclusion, the gradual increase in thrombogenicity after cessation of prasugrel treatment is due the increased activity of juvenile platelets.

\section{Introduction}

The autocrine mediator ADP is a moderately strong platelet agonist, stimulating platelet aggregation and thrombus formation and contributing to thrombus stabilization. ${ }^{1}$ At present, the ADP receptor $\mathrm{P}_{2} \mathrm{Y}_{12}$ is one of the most effective targets for antiplatelet therapy, i.e. by clopidogrel, prasugrel, and ticagrelor. ${ }^{2,3}$ Clopidogrel in combination with aspirin has been the standard therapy over the last decade to prevent recurrent atherothrombotic complications in patients who had a myocardial infarction. ${ }^{4}$ More recently, prescription of prasugrel or ticagrelor instead of clopidogrel is increasingly recommended following publication of the TRITON and PLATO studies, which demonstrated a net clinical benefit over clopidogrel on top of aspirin, due to a higher degree of platelet inhibition. ${ }^{4-9}$ The thienopyridines clopidogrel and prasugrel are both prodrugs, which require metabolic conversion to form an active metabolite that irreversibly interacts with the $\mathrm{P}_{2} \mathrm{Y}_{12}$ receptors of circulating platelets. ${ }^{10}$ In contrast, ticagrelor is a reversible, non-competitive $\mathrm{P}_{2} \mathrm{Y}_{12}$ antagonist - belonging to the cytopentyl-triazolo class of pyrimidines - interacting with the platelet receptors without metabolic conversion. ${ }^{10-12}$

Due to the irreversible inhibition of the platelet $P 2 Y_{12}$ receptors by the active metabolites of clopidogrel and prasugrel, the formation of new platelets is required to recover platelet function. ${ }^{7,9}$ After ticagrelor treatment, recovery of platelet function is only determined by the elimination time of the drug (half-life of 8 hours). ${ }^{10}$ There is limited evidence that during the offset period of irreversible $\mathrm{P}_{2} \mathrm{Y}_{12}$ inhibitors (usually 
prescribed for one year) patients may have an increased risk of a recurrent myocardial event. ${ }^{13-17}$ This may point to hyperactivity of the newly formed platelets in the offset period, although the mechanism is unclear.

Previous experiments with rats have indicated that stopping clopidogrel treatment resulted in the sudden appearance of a population of fully responsive platelets, whereas recovery from ticagrelor treatment led to a more gradual regain in function of all platelets. ${ }^{18}$ The newly formed, juvenile platelets with active $\mathrm{P} 2 \mathrm{Y}_{12}$ receptors seemed to preferentially incorporate into thrombi generated under flow conditions. ${ }^{18}$

Juvenile platelets, also described as reticulated platelets because of the presence of reticular-bound mRNA, are those platelets that are shed most recently from the megakaryocytes in the bone marrow. ${ }^{19-21}$ Due to the gradual degradation of mRNA, they form only a small part of the entire platelet population. Only little is known about the properties of reticulated platelets, although incidental studies report on a larger size with more granules, ${ }^{22}$ and a high reactivity towards platelet agonists. ${ }^{23,}{ }^{24}$ With a Sysmex analyzer, using cell-permeant fluorescent mRNA dyes containing polymethine and oxazine, or using the mRNA probe thiazole orange, evidence has been obtained that patients with more reticulated platelets respond less effective to clopidogrel or prasugrel medication. ${ }^{25-27}$

In the present study, we investigated how the hemostatic function of platelets recovers after discontinuation of prasugrel treatment. We hypothesized an immediate recovery of newly formed, juvenile platelets to the level of untreated platelets. Our data provide evidence for a critical role of the juvenile platelets in the regained aggregation of platelets and thrombus formation, and also show that these platelets gradually increase in responsiveness.

\section{Methods}

For a detailed description of the methods, please see the supplementary material.

\section{Patients and control subjects}

This study was approved by the local medical ethics committee (MEC 12-3-075). All patients and healthy volunteers gave written informed consent for participation according to the Helsinki declaration. Sixteen patients were studied who were treated with prasugrel (10 mg/day) for one year and long-term aspirin (80-100 mg/day) due to a myocardial infarction with ST elevation. After one year of prasugrel treatment, blood was collected on the last treatment day, and at 1, 2, 5 and 30 days later. From two patients, blood samples were also collected after 12 days to better understand the delayed regain of platelet function. Patients with a malignancy, active infection or a known platelet disorder were not included. Blood was obtained by venipuncture into Vacuette tubes, containing $\mathrm{K}_{2}$-EDTA, for measurement of hemostatic variables and immature platelet fraction (IPF) using a Sysmex XN-9000 analyzer (Sysmex, Chuo-ku Kobe, Japan); 3.2\% 
$(w / v)$ trisodium citrate for platelet function measurements, or hirudin for whole blood platelet aggregation. Control experiments were performed with blood drawn from healthy volunteers collected in trisodium citrate or acidic citrate dextrose. ${ }^{28}$

Preparation of platelet-rich plasma, platelets and red cells

Platelet-rich plasma (PRP), platelet-free plasma and washed platelets were prepared as described. ${ }^{29}$ Platelet counts were determined with a thrombocounter XP300 Sysmex analyzer (Sysmex, Chuo-ku Kobe, Japan). Washed red blood cells were prepared as shown before. ${ }^{30}$

Irreversible $P 2 Y_{12}$ inhibition in vitro

PRP from healthy donors was treated with lysine aspirin. ${ }^{28}$ The platelets were incubated with the active metabolite of clopidogrel (CAM) or vehicle medium. Mixtures of washed CAM-treated and vehicle-treated platelets were used for measurement of platelet aggregation, integrin $\alpha_{\mathrm{llb}} \beta_{3}$ activation by flow cytometry and perfusion experiments with reconstituted whole blood.

\section{Platelet aggregation}

Aggregation of platelets in PRP was measured using a Chronolog aggregometer (Stago, Asnières sur Seine Cedex, France). ${ }^{31}$ Aggregation of platelets in whole blood was measured by Multiplate impedance aggregometry (Roche Diagnostics, Basel, Switzerland) as described. ${ }^{32}$ Ticagrelor $(1 \mu \mathrm{M})$, being more potent than prasugrel ${ }^{33}$, was added in vitro to block residual $\mathrm{P}_{2} \mathrm{Y}_{12}$ activity, where indicated.

\section{Flow cytometric analysis of platelet subpopulations}

Flow cytometry was performed on an Accuri $\mathrm{C} 6$ flow cytometer with CFlow Plus software (Becton-Dickinson Bioscience). To check for integrin $\alpha_{1 \mathrm{lb}} \beta_{3}$ activation, platelets were activated with $2 \mathrm{MeS}$-ADP in the presence of FITC-conjugated PAC-1 antibody against the activated $\alpha_{\| l b} \beta_{3}$ integrin. Activated platelets were identified as before. ${ }^{31}$ Ticagrelor (1 $\mu \mathrm{M})$ was added, where indicated.

Juvenile platelets were identified using two different methods of mRNA staining, i.e. with thiazole orange ${ }^{34}$ or by a novel method using Cy5-labeled oligo-dT, which binds to the poly-A tail of mRNA species. Thiazole orange (15\% in filtered PBS) was added to PRP, according to established procedures. ${ }^{34}$ Samples were activated with 2MeS-ADP in the presence of AF647-fibrinogen. For staining with $5^{\prime} \mathrm{Cy} 5$-oligo-dT, washed platelets were activated with 2MeS-ADP in the presence of OG488-fibrinogen. Samples were fixed with $0.2 \%$ formaldehyde, permeabilized with $0.1 \%$ saponin and subsequently incubated with $5^{\prime}$-Cy5-oligo-dT at $37^{\circ} \mathrm{C}$. For all samples, $5^{\prime} \mathrm{Cy} 5$-oligo-dA was used as a negative control probe to check for specificity of the staining. Color compensation was not required as fluorescent spectra did not overlap.

The average percentage of juvenile platelets as analyzed by the thiazole orange staining and the oligo-dT staining was $6.7 \%( \pm 1.9 \%)$ and $21.5 \%( \pm 5.8 \%)$ respectively. The discrepancy in the percentage of detected juvenile platelets can be explained by 
the higher sensitivity of the oligo-dT staining to detect mRNA in comparison to thiazole orange. In order to use a uniform definition of juvenile platelets, the threshold for juvenile platelets was based on the IPF as determined by the Sysmex XN9000 analyzer, which is an internationally validated method in the clinic. An alternative analysis of juvenile platelets, based on the negative controls of both stainings, is presented in the supplements (Suppl. Fig. 3).

\section{Thrombus formation in whole blood}

Whole blood thrombus formation on microspots in a parallel-plate flow chamber was measured, basically as described before. ${ }^{35}$ Patient blood samples were perfused through the chamber for 4 minutes at a wall-shear rate of $1600 \mathrm{~s}^{-1}$, while 2MeS-ADP (0.1 $\mathrm{\mu M}$, f.c.) was co-perfused with a second pump. Ticagrelor was added where indicated. Thrombi were stained with AF647-labelled fibrinogen and, when indicated, with DiOC $_{6} .{ }^{35}$ Brightfield and fluorescence microscopic images were captured with an EVOS fluorescence microscope, equipped with a 60x oil objective. Images were analyzed using Metamorph (Molecular Devices, Sunnyvale CA, USA) and ImageJ (open access) software. ${ }^{35}$

For measurement of thrombus formation of reconstituted blood samples from healthy controls, mixtures of CAM- and vehicle-treated platelets were added to washed red cells and plasma. In these experiments, the CAM-treated and vehicle-treated platelets were pre-labeled with the membrane probes CellVue Maroon and PKH26 respectively. Microscopic DIC and confocal fluorescent images were taken using a Zeiss LSM7 microscope (Oberkochen, Germany). ${ }^{35}$

\section{Statistical analysis}

Statistical analysis was performed using the SPSS Statistics 22 package (Armonk, NY, USA). Statistical analysis was performed using a one-way-repeated-measures-ANOVA or with a Friedman test with a post hoc Wilcoxon signed rank test. Bonferroni correction was applied when comparing multiple groups.

\section{Results}

\section{$P 2 Y_{12}$-inhibited platelets participate less in thrombus formation}

In order to determine how platelets with non-responsive $\mathrm{P}_{2} \mathrm{Y}_{12}$ receptors interact with responsive platelets in aggregation and thrombus formation, platelets from control subjects were treated with the clopidogrel active metabolite (CAM) and mixed in various proportions with untreated platelets. All platelets were also treated with aspirin, in order to mimic conditions as in patients (see below). Flow cytometric analysis indicated that, upon stimulation with ADP, these platelet mixtures formed two distinct populations in terms of activation of integrin $\alpha_{11 b} \beta_{3}$ and binding of OG488-fibrinogen. The population of fibrinogen-binding platelets decreased with increasing fractions of CAMtreated platelets (Fig. 1A and Suppl. Fig. 1A). Addition of the P2Y $\mathrm{Y}_{12}$ receptor antagonist ticagrelor decreased the population of fibrinogen-binding platelets to the level of $100 \%$ 
A

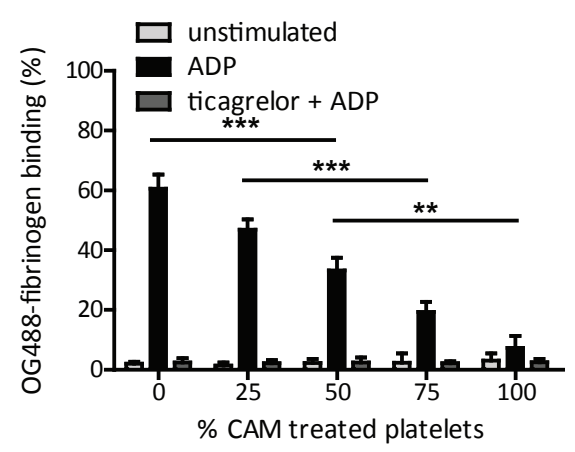

C

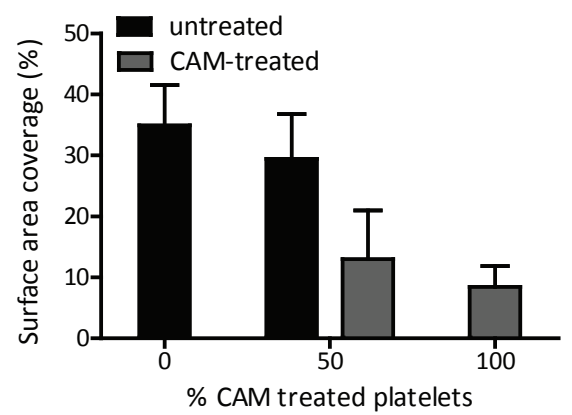

B

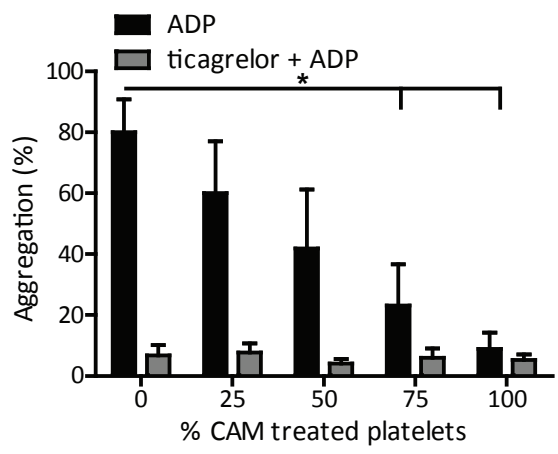

D

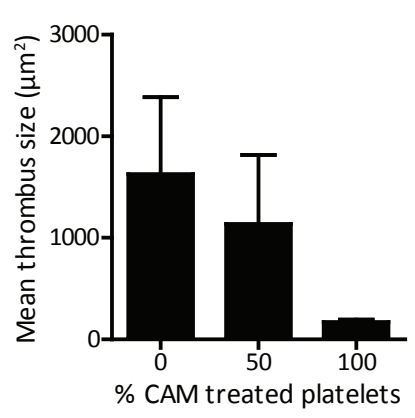

Figure 1. Impaired aggregation and contribution to thrombus formation of $P 2 Y_{12}$-inhibited platelets. (A, B) Different mixtures of uninhibited platelets and CAM-treated platelets were preincubated with vehicle or $1 \mu \mathrm{M}$ ticagrelor, and stimulated with $20 \mu \mathrm{M}$ 2MeS-ADP. (A) Fractions of OG488-fibrinogen binding platelets, representative for uninhibited platelets with activated integrin $\alpha_{116} \beta_{3}$ (flow cytometry). (B) Maximal aggregation (\% change in light transmission) within 5 minutes. (C, D) Reconstituted blood with different fractions of CAM-treated platelets was perfused 4 minutes over collagen at $1600 \mathrm{~s}^{-1}$ in the presence of $2 \mathrm{MeS}$ ADP. Uninhibited platelets were prelabeled with PKH26 and CAM-treated platelets with CellVue Maroon; for images see Suppl. Figure 2. (C) Quantification of the surface area covered by the populations of labelled platelets. (D) Mean thrombus size. Means \pm SD $(n=3), *_{p}<0.05, * * p<0.01, * * * p<0.001$ (ANOVA with Bonferroni correction).

CAM-treated platelets (Fig. 1A), indicating that the CAM treatment had fully blocked the $\mathrm{P}_{2} \mathrm{Y}_{12}$ receptors. Light transmission measurements indicated a gradual decrease in ADP-induced platelet aggregation, when the fraction of CAM-treated platelets increased (Fig. 1B). Addition of ticagrelor again antagonized the remaining aggregation response. Together, these data suggest that the reduced integrin activation of the CAM-treated platelets prevented their incorporation into aggregates.

To investigate this further, we assessed how the CAM-treated platelets participated in thrombus formation on immobilized collagen under high-shear flow conditions. Therefore, the $\mathrm{P}_{2} \mathrm{Y}_{12}$-inhibited platelets were labelled with the red-exciting membrane label CellVue Maroon, whereas the untreated platelets were labeled with the greenexciting membrane label PKH26. This labeling did not affect platelet activation responses 
(data not shown). Mixtures with $0 \%, 50 \%$ or $100 \%$ of CAM-treated platelets were added to red blood cells and plasma from the same donor to obtain reconstituted blood with different proportions of $\mathrm{P}_{2} \mathrm{Y}_{12}$-inhibited platelets. In comparison to reconstituted blood with solely uninhibited platelets, increasing proportions of CAM-treated platelets had limited impact on platelet adhesion to the collagen surface, but markedly suppressed the formation of large platelet aggregates (Suppl. Fig. 2). As a result, with CAM-treated platelets, surface area coverage (Fig. 1C) and mean thrombus size (Fig. 1D) on collagen progressively decreased. Strikingly, with $50 \%$ of CAM-treated platelets, about $30 \%$ of the

A

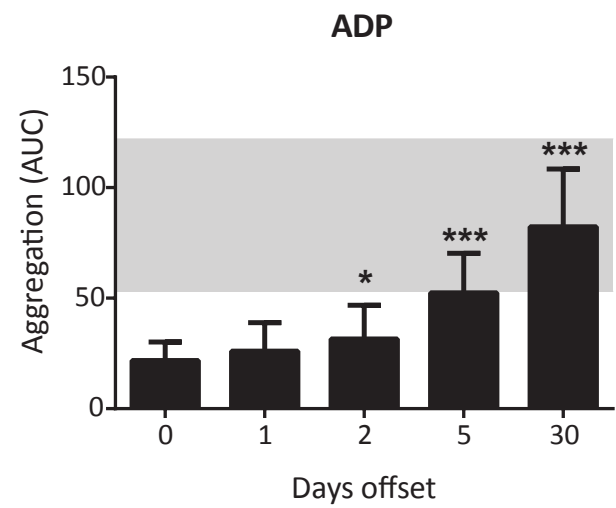

C

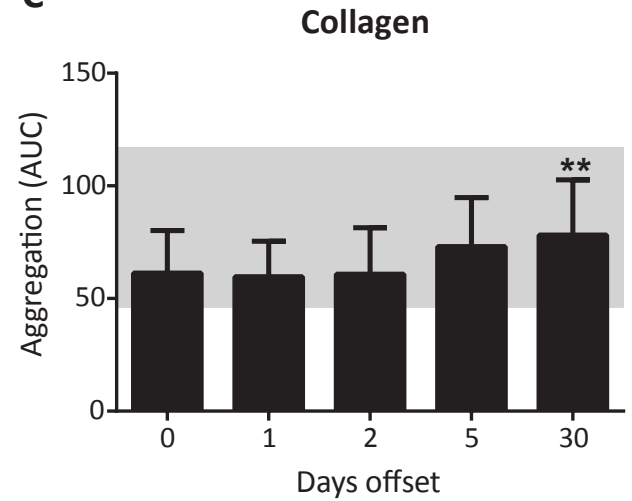

B

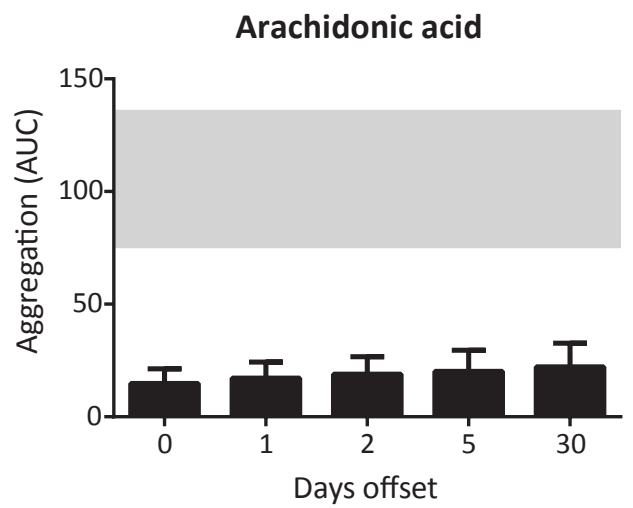

D

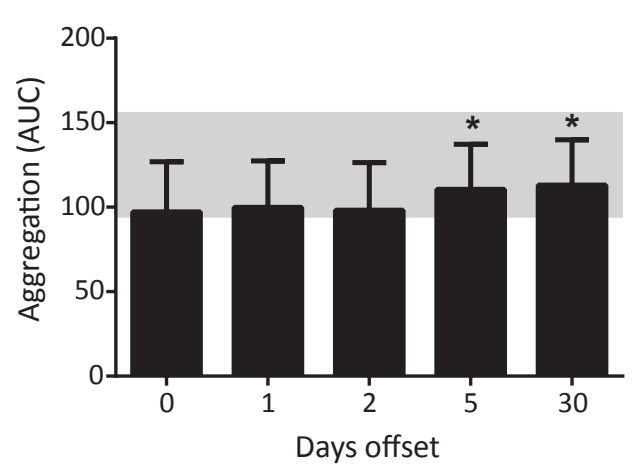

Figure 2. Partial restoration of platelet aggregation in whole blood upon prasugrel offset. Whole blood samples from patients (at indicated days after stopping prasugrel intake) were stimulated with $6.4 \mu \mathrm{M}$ ADP (A), $0.5 \mathrm{mM}$ arachidonic acid (B), $3.2 \mu \mathrm{g} / \mathrm{ml}$ collagen (C), or $32 \mu \mathrm{M}$ TRAP (D). Measurements by Multiplate impedance aggregometry; outcome expressed as the area under the impedance curve (AUC). Grey blocks indicate normal ranges, established for healthy subjects. Means \pm SD $(n=15-16),{ }^{*} p<0.05,{ }^{* *} p<0.01, * * * p$ $<0.001$ vs. day 0 (ANOVA with Bonferroni correction). 

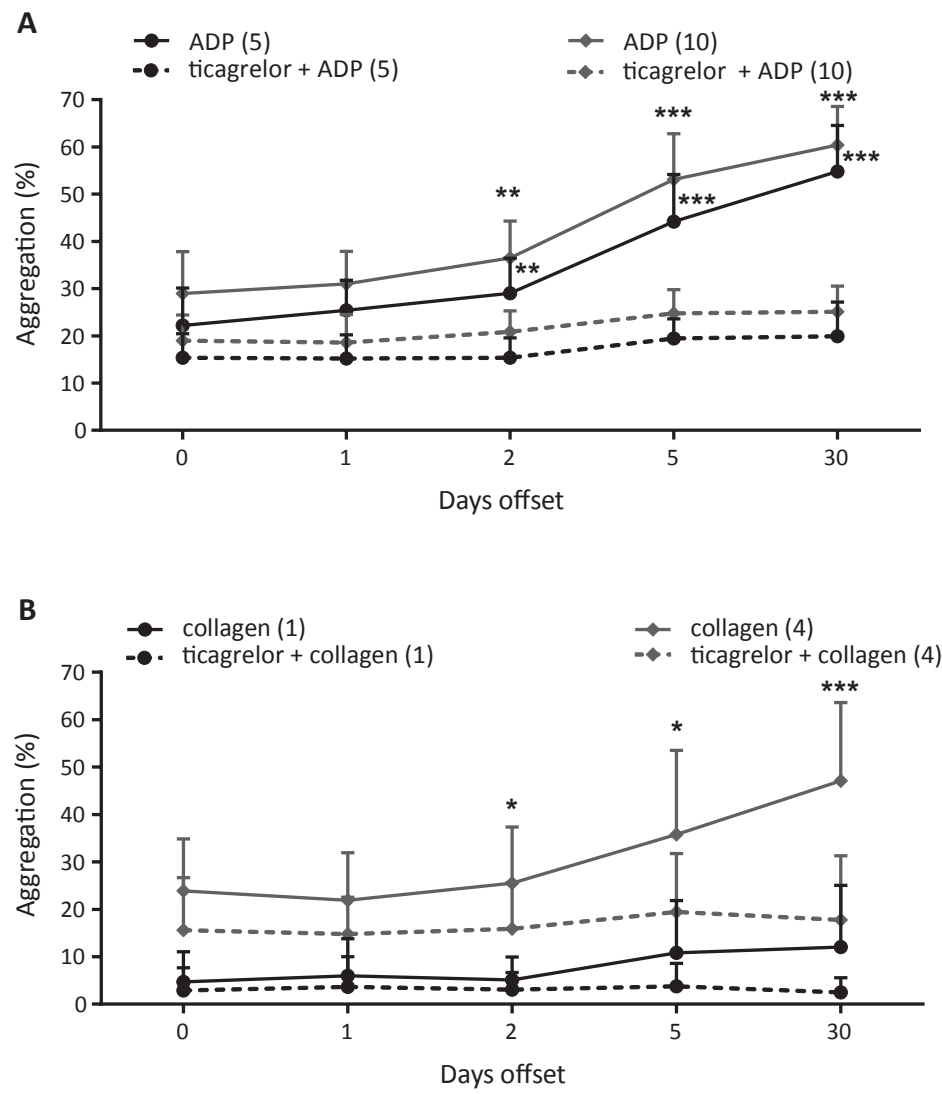

Figure 3. Restored ADP- and collagen-induced aggregation of platelets upon prasugrel offset. Patient PRP (at indicated days after stopping prasugrel intake) activated with 5-10 $\mu \mathrm{M}$ ADP (A) or 1-4 $\mu \mathrm{g} / \mathrm{ml}$ collagen (B),

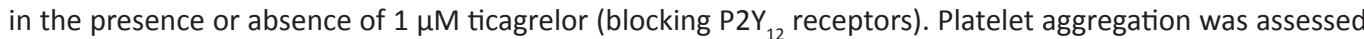
by light transmission aggregometry (\% change in maximal light transmission). Means $\pm S D(n=16), * p<0.05$, $* * p<0.01$ and ${ }^{* * *} p<0.001$ vs. day 0 (ANOVA with Bonferroni correction).

surface area coverage was occupied by the $\mathrm{P} 2 \mathrm{Y}_{12}$ inhibited platelets, while the remaining $70 \%$ was occupied by the $\mathrm{P}_{2} \mathrm{Y}_{12}$-responsive platelets. Together, these results indicate that

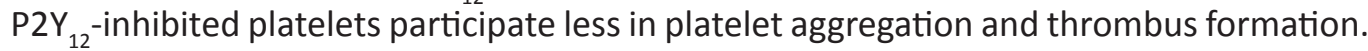

\section{Gradual restoration of platelet aggregation in patients upon prasugrel offset}

The offset phase of prasugrel medication was studied in 16 patients. The patients had a mean age of $59 \pm 9$ years (mean $\pm S D$ ); three patients were diagnosed with type II diabetes mellitus (Suppl. Table 1). Blood samples taken at day 0 (i.e. last day of prasugrel intake) showed a normal hematocrit of $0.435 \pm 0.035 \mathrm{l} / \mathrm{l}$ and platelet count of $239 \pm 81$ $\times 10^{9} /$ l. Subsequent blood samples were taken at days $1,2,5$, and 30 , and no noticeable changes in hematocrit or platelet count were observed.

Measurements of whole blood aggregation (Multiplate assay) showed a gradual increase in ADP-induced aggregation upon offset from days 2 to 30 (Fig. 2A). Interestingly, the aggregation response further increased at day 30 in comparison to day 5 . Whole 
A

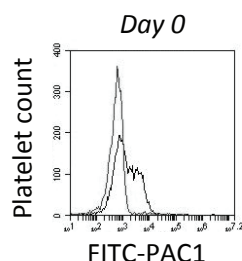

FITC-PAC1

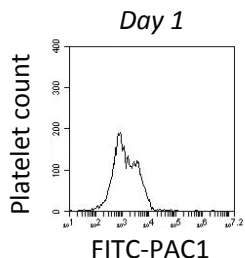

FITC-PAC1

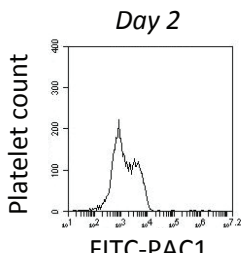

FITC-PAC1

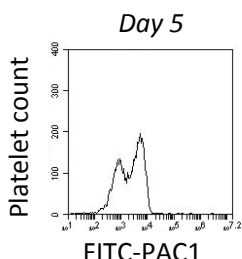

FITC-PAC1

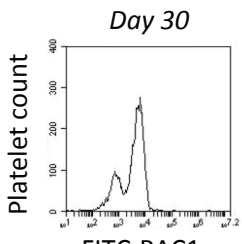

FITC-PAC1
B

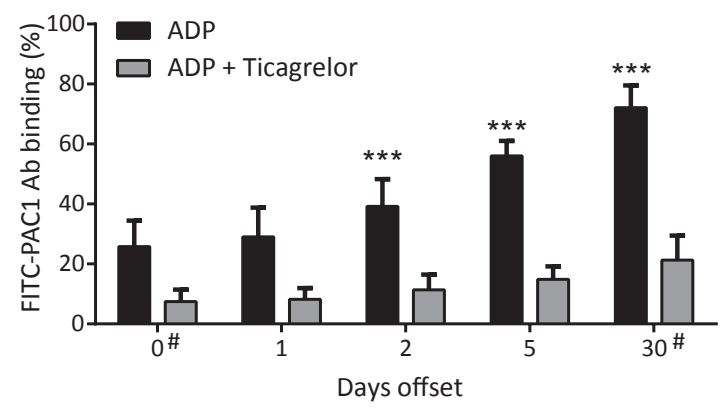

C

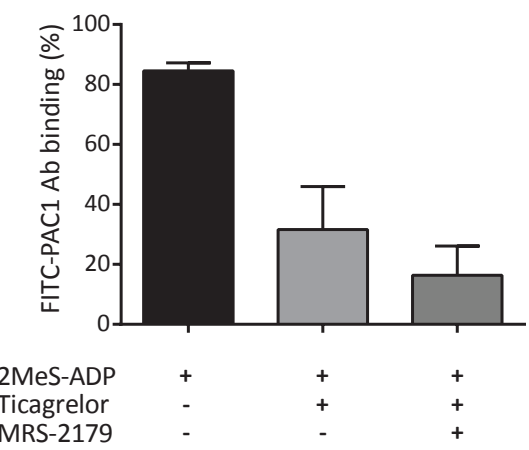

Figure 4. Reappearance of ADP-responsive platelets upon prasugrel offset. Whole blood from patients or healthy controls was preincubated with vehicle, $1 \mu \mathrm{M}$ ticagrelor or $50 \mu \mathrm{M}$ MRS-2179 (as indicated), and then stimulated with $1 \mu \mathrm{M} 2 \mathrm{MeS}$-ADP. Activation of $\alpha_{111} \beta_{3}$ was assessed by flow cytometric analysis using FITClabelled PAC-1 mAb. (A) Representative histograms of PAC-1 mAb binding to patient platelets stimulated with ADP (black) or unstimulated (grey) during offset. (B) Quantification of positive platelets. (C) Flow cytometric analysis of healthy control platelets. Means $\pm S D(n=16), * * * p<0.001$ vs. day 0 ADP (ANOVA with Bonferroni correction); ${ }^{n} \mathrm{n}=15$.

blood aggregation in response to arachidonic acid remained below the normal range (Fig. 2B), thus confirming that all patients still used aspirin during the offset period. The aggregation responses to collagen and thrombin receptor-activating peptide (TRAP) were within the normal ranges, but slightly increased at later sampling points (Fig. 2CD). Similarly, light transmission aggregometry in PRP indicated a restoration in ADPinduced platelet aggregation from day 2 onward (Fig. 3A). In this test, the aggregation response with two ADP concentrations was near maximal already at day 5 . Collageninduced platelet aggregation also significantly improved, but only at later time points (Fig. 3B). Control experiments in the presence of ticagrelor showed that the increase in aggregation during prasugrel offset was fully antagonized, confirming that the regained platelet reactivity was fully due to increased $\mathrm{P}_{2} \mathrm{Y}_{12}$ receptor function (Fig. 3A-B).

Formation of a highly reactive population of juvenile platelets upon prasugrel offset

Based on earlier experiments with rats, ${ }^{18}$ we expected during the offset phase of prasugrel medication the rapid formation of a population of newly formed, fully $\mathrm{P}_{2} \mathrm{Y}_{12}$-responsive platelets. A pertinent question was how the regained response in 
A

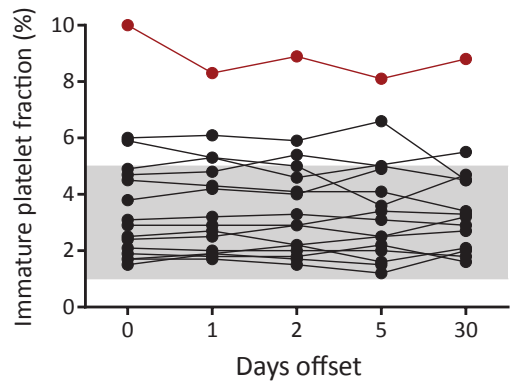

B

Thiazole orange

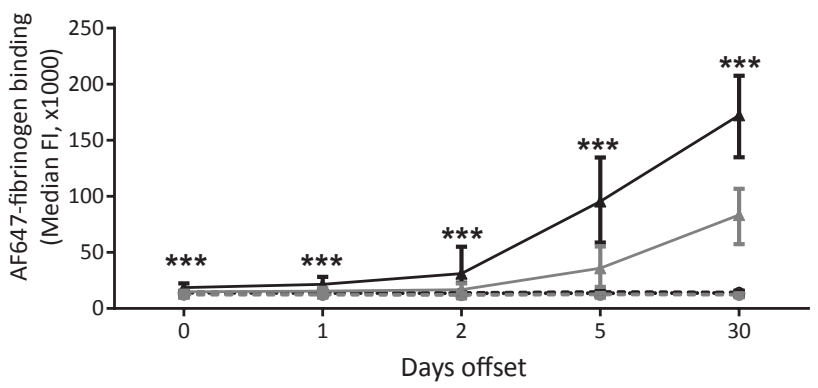

TO positive

- unstimulated

$\mp$ ADP

- t ticagrelor + ADP

TO negative

- unstimulated

$\leftarrow$ ADP

- ticagrelor + ADP

C

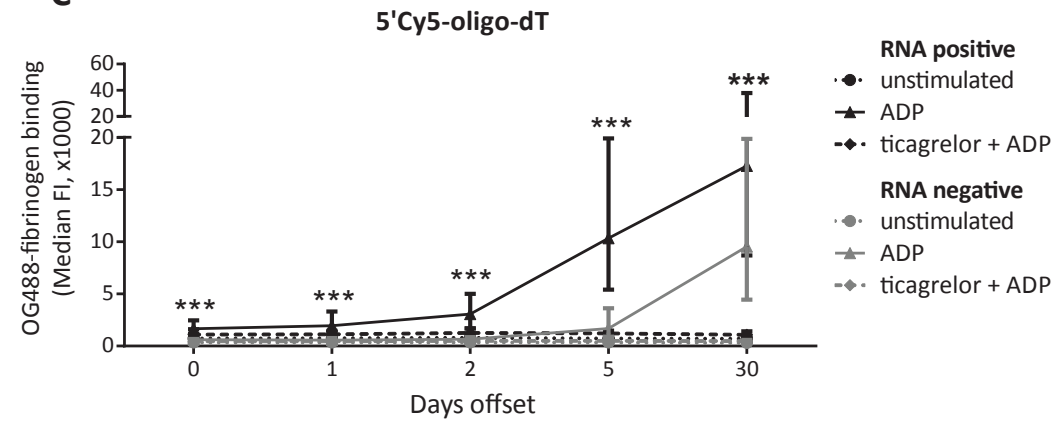

Figure 5. Increased ADP-induced $\alpha_{1 \mathrm{~b}} \beta_{3}$ activation of juvenile platelets formed upon prasugrel offset. Platelets from 16 patients during offset from prasugrel were activated and analyzed by flow cytometry, with per sample a gating for juvenile platelets based on the immature platelet fraction (Sysmex XN-9000 analyzer). (A) Immature platelet fraction for each of the patients during offset, as determined with a Sysmex XN-9000 analyzer. Red dots are from patient with a high immature platelet fraction (IPF $=8.8 \%$ ). Juvenile platelets were identified by staining with thiazole orange or 5'Cy5-oligo-dT. (B) PRP was stained with thiazole orange and activated with $1 \mu \mathrm{M} 2 \mathrm{MeS}-\mathrm{ADP}$ in the presence of AF647-fibrinogen. Shown is extent of activated $\alpha_{11 b} \beta_{3}$ of thiazole-positive and -negative platelets, as assessed from fibrinogen binding. (C) Washed platelets were activated with $1 \mu \mathrm{M}$ 2MeS-ADP in the presence of OG488-fibrinogen. The cells were subsequently fixed and permeabilized with saponin to allow staining of mRNA by incubation with 5'Cy5-oligo-dT. Negative control stains were performed with $5^{\prime} C y 5$-oligo-dA. Shown is extent of $\alpha_{\| b} \beta_{3}$ activation of mRNA-positive and -negative platelets, as determined from fibrinogen binding. Medians \pm IQR $(n=16),{ }^{* * *} p<0.001$ vs. corresponding mature platelet fraction (Friedman test). 
P2Y $\mathrm{Y}_{12}$ receptor activity was linked to the appearance of this new platelet population. To investigate this, flow cytometry was used to analyze platelets stimulated with the stable (nucleotidase-resistant) ADP analogue, 2MeS-ADP, for binding of FITC-labeled PAC-1 antibody, indicative of integrin $\alpha_{11 \mathrm{~b}} \beta_{3}$ activation. At day 0 , a limited fraction of $26 \pm 9 \%$ of the platelets showed activated $\alpha_{11} \beta_{3}$, and this fraction (recognized as a separate peak in the histograms) gradually increased to $56 \pm 5 \%$ at day 5 and $72 \pm 7 \%$ at day 30 (Fig. 4A-B). In comparison, activation of aspirin-treated platelets from healthy control subjects with $2 \mathrm{MeS}-\mathrm{ADP}$ resulted in a similar percentage of platelets with activated $\alpha_{1 \mathrm{lb}} \beta_{3}$ as platelets collected at 30 days after prasugrel cessation (Fig. 4C). In platelets from patients (Fig. 4B) and healthy controls (Fig. 4C), the fraction of platelets binding FITC-PAC1 antibody was

A

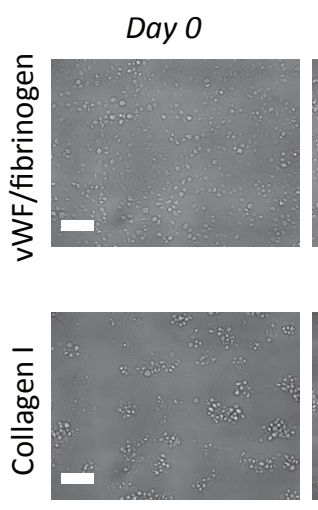

B

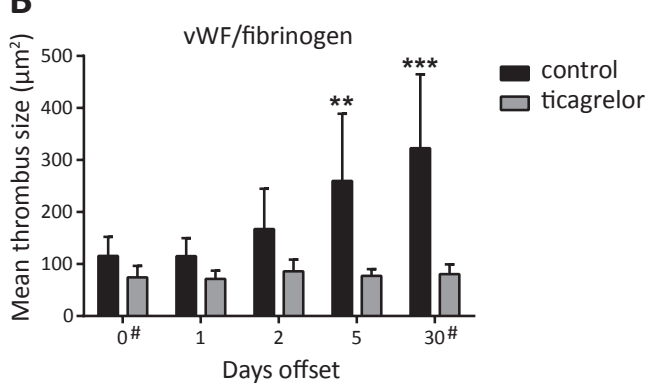

Day 2
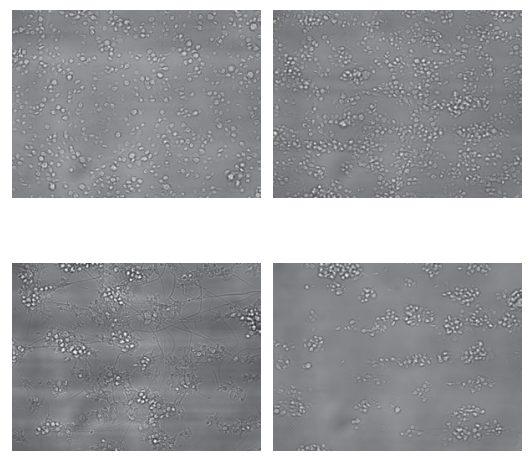

C

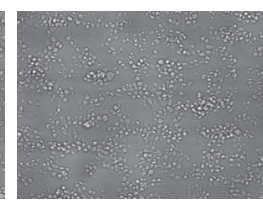

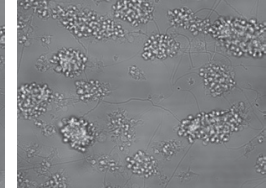
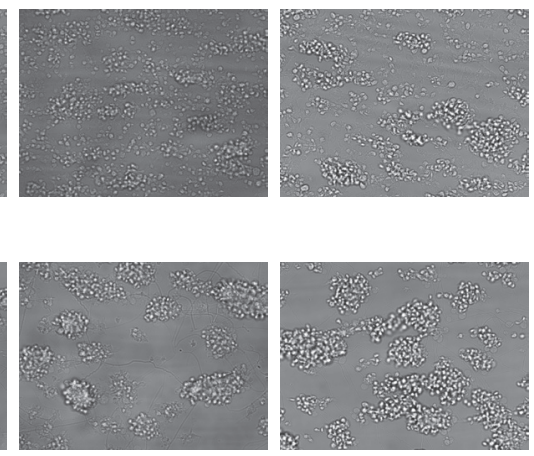

Day 30

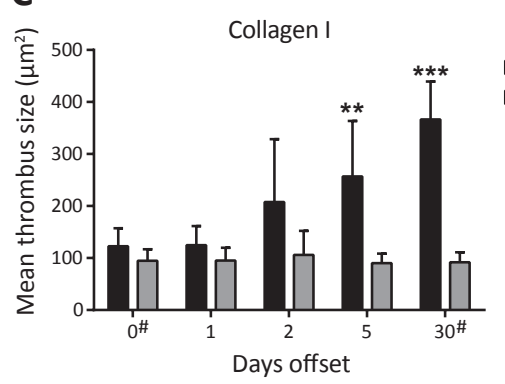

Figure 6. Restored thrombus formation under flow upon prasugrel offset. Whole blood samples from patients (at indicated days after stopping prasugrel intake) were co-perfused with $0.1 \mu \mathrm{M} 2 \mathrm{MeS}$-ADP over microspots containing VWF/fibrinogen or collagen type I, at a shear rate of $1600 \mathrm{~s}^{-1}$ for 4 minutes. Vehicle (control) or $10 \mu \mathrm{M}$ ticagrelor was added to the blood. (A) Brightfield images of thrombi from a representative patient formed on the two microspots. Bars $=25 \mu \mathrm{m}$. Assessment of mean thrombus size for surfaces with vWF/fibrinogen (B) or collagen I (C). Means \pm SD $(n=16), * * p<0.01, * * * p<0.001$ vs. day 0 (ANOVA with Bonferroni correction); ${ }^{*} \mathrm{n}=15$. 
greatly, but incompletely reduced by the addition of ticagrelor. Co-incubations with the $\mathrm{P} 2 \mathrm{Y}_{1}$ receptor antagonist MRS-2179 indicated that the residual $\alpha_{\mathrm{II}} \beta_{3}$ activation was most likely due to activation via $\mathrm{P} 2 \mathrm{Y}_{1}$ receptors.

Several assays were performed to determine whether the accumulating platelets with activated $\alpha_{11 b} \beta_{3}$ indeed consisted of newly formed, juvenile platelets. Therefore, platelet mRNA was quantified using two different mRNA probes: thiazole orange as an established, but weak fluorescent mRNA dye $\mathrm{e}^{22}$; and 5'Cy5-labeled oligo-dT, binding to the platelet mRNA poly-A tails ${ }^{21}$, which was added to pre-activated and permeabilized platelets. Supplemental figure 3 shows typical dotplots of unstimulated and ADPactivated platelets stained with the $5^{\prime} \mathrm{Cy} 5$-oligo-dT (Suppl. Fig. 3A-B) or thiazole orange (Suppl. Fig. 3C-D). Juvenile and old platelets were discriminated per blood sample, based on the negative control and the immature platelet fraction determined by Sysmex XN-9000 (Fig 5A). For the majority of the patients, with one noticeable exception, the immature platelet fraction was in the normal range, with mean values of $3.6 \pm 1.9 \%$, and remaining constant per patient during the study (Fig. 5A). Markedly, with either mRNA probe (thiazole orange or $5^{\prime} \mathrm{Cy} 5$-oligo-dT), the fraction of platelets with positive staining showed increased fibrinogen binding following ADP stimulation from day 2 on (Fig. 5BC). Fibrinogen binding ( $\alpha_{11 b} \beta_{3}$ activation) of this juvenile platelet population continued to increase from day 5 to day 30 . The difference between 5 and 30 days was significant for thiazole orange $(p=0.003)$, and was borderline significant for 5 'Cy5-oligo-dT $(p=0.063)$, thus suggesting partial inhibition of these platelets even after 5 days offset. With either mRNA probe, the fraction of (older) platelets with negative staining was substantially lower in fibrinogen binding, with noticeable increase of activated integrins only from day 5 onwards (Fig. 5B-C). The difference in fibrinogen binding between juvenile and older platelets was significant at all days $(p<0.001)$. These results were confirmed using an alternative analysis based on the appropriate negative controls (Suppl. Fig 3.) Here again, the reactivity of juvenile platelets, expressed as activation ratio, was significantly higher in comparison to mature platelets at all days. Moreover, this analysis also shows the increasing reactivity of juvenile platelets over time for both stainings (Suppl. Fig 4A). Additional platelet measurements with a limited number of patients showed that, after 12 days of prasugrel discontinuation, the reactivity of juvenile platelets was in between the day 5 and 30 values (Suppl. Fig. 4B). Interestingly, in patients with a high immature platelet fraction (IPF $\geq 7.0 \%$ ), the population of juvenile platelets showed a faster increase in fibrinogen binding, being near maximal already at day 5 of prasugrel offset (Suppl. Fig. $4 A-B)$.

\section{Gradual increase in thrombus size upon prasugrel offset}

To investigate whether the increased reactivity of juvenile platelets after prasugrel cessation translates into enhanced thrombus formation, whole blood was perfused over microspots containing vWF/fibrinogen or type I collagen. ${ }^{35}$ Given the major role of P2Y ${ }_{12}$ signaling in thrombus build-up ${ }^{36}$, we determined thrombus size at the different time points. Regardless of the surface, at day 0 many single platelets and small aggregates were detected, whilst at later offset days larger aggregates were formed. From the recorded brightfield images it was apparent that the thrombi at days 5 and 30 displayed 
a more contracted morphology (Fig. 6A). Quantification of the feature size showed on both microspots a progressive increase in mean thrombus size during offset (Fig. 6B-C). On the other hand, platelet adhesion to VWF/fibrinogen or collagen was not changed between day 0 and 30, as surface area coverage (by single platelets and aggregates) remained similar (Suppl. Fig. 5A-B).

Staining of thrombi with AF647-labeled fibrinogen allowed assessment of integrin $\alpha_{110} \beta_{3}$ activation. On both the vWF/fibrinogen and collagen microspots, a marked increase in fibrinogen binding to the aggregated platelets was detected for the later blood samples (Suppl. Fig. 6A-B). Addition of ticagrelor to the blood resulted in a nearly complete abolition of platelet aggregation at all offset days, but did not block platelet adhesion. With blood samples from a limited number of patients, staining with DiOC made it possible to record $z$-stacks by confocal microscopy for 3D visualization of the thrombi. This illustrated the gradual increase in thrombus size from day 0 to day 5 and 30 (Suppl. Fig. 6C). Taken together, these results indicate that the accumulation of highly $\mathrm{P} 2 \mathrm{Y}_{12}$-responsive, juvenile platelets upon prasugrel offset led to a gradual increase in formation of large-size thrombi.

\section{Discussion}

In this paper, we confirm earlier findings $7,9,15,16,37$ that when treatment with an irreversible $\mathrm{P} 2 \mathrm{Y}_{12}$ antagonist is stopped, platelet aggregation in response to ADP gradually recovers in time, when measured by light transmission aggregometry. Near maximal aggregation after stopping prasugrel intake was already reached at day 5 , which is in line with results from earlier trials, such as Recovery showing recuperation of this platelet response after 7-9 days of prasugrel cessation. ${ }^{9}$ However, we also found that other platelet function tests, such as ADP-induced whole blood aggregation and integrin $\alpha_{11 \mathrm{~b}} \beta_{3}$ activation were incompletely recovered at day 5 in comparison to day 30 . In vitro addition of ticagrelor completely antagonized the time-dependent increase in platelet responses, thereby proving that the recuperation was due to regained $\mathrm{P}_{2} \mathrm{Y}_{12}$ signaling.

Detailed flow cytometric analysis indicated that the functional recovery during prasugrel offset was caused by the appearance of a population of juvenile platelets that was increasingly responsive towards ADP. Separation of newly formed and older platelets with two mRNA probes, thiazole orange and a new Cy5-conjugated oligo-dT probe, revealed increased responsiveness to ADP of the positively stained platelet population in terms of integrin $\alpha_{11 b} \beta_{3}$ activation and fibrinogen binding. However, both probes also gave unexpected results. First, we observed a marked increase in ADP responsiveness of the juvenile platelet population after only two days of offset, and for the older platelet population after five days. This suggested that the majority of juvenile platelets formed during the first days still had inhibited $\mathrm{P}_{2} \mathrm{Y}_{12}$ receptors, taking into account the presence of the prasugrel active metabolite in the circulation for 7-8 hours post prasugrel administration..$^{38}$ Second, we found a steady rise in the responsiveness of juvenile platelets up to days 12 and 30 of offset. This also points towards residual ADP receptor inhibition of new platelets, likely at the megakaryocytic level, for more than 5 days. In agreement with this hypothesis, experimental animal models have shown that prasugrel is present in the bone marrow. ${ }^{39}$ An alternative explanation for this phenomenon might be a long- 
term increase in autocrine platelet-stimulating effects due to the larger population of P2Y ${ }_{12}$-responsive platelets. ${ }^{3,40}$ However, flow cytometric analysis did not point to a higher extent of integrin $\alpha_{111} \beta_{3}$ activation of the ADP-responsive platelet population as a whole.

To determine how the increased reactivity of juvenile platelets translates into hemostasis, we studied thrombus formation under flow on two different adhesive surfaces using whole blood. Platelet deposition and aggregate formation on the VWF/ fibrinogen and the collagen surfaces restored during the offset and was only maximal at day 30. The regained $\mathrm{P} 2 \mathrm{Y}_{12}$ activity was most apparent from thrombus size, with larger thrombi towards the end phase of the offset. This is in agreement with earlier work showing that signaling via $\mathrm{P}_{2} \mathrm{Y}_{12}$ is crucial for thrombus formation and stabilization. ${ }^{1}$, ${ }^{36}$ Also others found a relative preponderance of thiazole orange-stained platelets in a thrombus. ${ }^{41}$

In recent years, it has been debated whether the termination of clopidogrel or prasugrel intake leads to a rebound effect of recurrent cardiovascular events, perhaps related to platelet hyper-reactivity. Several research groups did report a rebound effect within 90 days after cessation of clopidogrel, ${ }^{13-17}$ while other researchers could not confirm this. ${ }^{37,42-44}$ This discussion has led to clinical trials investigating the effect of tapering clopidogrel medication with the idea to prevent platelet hyper-reactivity, but with no beneficial effect so far. ${ }^{42,43,45}$ The present work may explain this ambiguity in clinical offset effects. On the one hand, cessation of clopidogrel or prasugrel medication will lead to the appearance of the newly formed platelets with uninhibited $\mathrm{P}_{2} \mathrm{Y}_{12}$ receptors, which preferentially partake in thrombus formation. On the other hand, as shown in this study, at least during the first few days these juvenile platelets do not appear to be hyperactive, possibly due to residual receptor blockage at the megakaryocyte level. When tapering the medication, the prolonged time interval between two consecutive dosages will result in the alternative formation of uninhibited and inhibited platelets.

The present study has potential limitations, as we have investigated a relative small number of patients. Further, in our initial ex vivo studies we used the active metabolite of clopidogrel. Although prasugrel is a more potent $\mathrm{P}_{2} \mathrm{Y}_{12}$ antagonist in comparison to clopidogrel, ${ }^{5}$ we added the active metabolite of clopidogrel at concentrations high enough for maximal inhibition.

Patients on dual antiplatelet therapy who require surgery are recommended to stop prasugrel intake 7 days beforehand..$^{9}$ Our findings that prasugrel can still affect the reactivity of juvenile platelets during several days after treatment cessation does not plea for a shortening of this period. The compromised reactivity of juvenile platelets during the initial days of offset can contribute to a risk of bleeding upon surgery. When urgent surgery is required or when bleeding has to be controlled, platelet transfusions have shown to be effective in restoring haemostasis at 6 hours after a loading dose of prasugrel. ${ }^{46}$ Altogether, the present study provides clinically relevant detailed insights into the mechanisms of prasugrel offset, and thereby provides better insight into the optimal treatment regimen of $\mathrm{P}_{2} \mathrm{Y}_{12}$ inhibitors. 


\section{Acknowledgements}

The authors thank S. Lelieveld and the central diagnostic laboratory for technical support and R. Dennert for assisting in patient recruitment.

\section{Authorship and disclosures}

Authorship: CCFMJB performed experiments, analyzed data and drafted the paper; RW, JPVG, FS, and SMdW performed experiments; LFV and HC recruited the patients; YMCH contributed analytical tools; SN and JJJvG designed research and drafted the paper; JMWH and PEJvdM designed research and drafted and finalized the paper.

Conflict of interest disclosure: Parts of this study were financially supported by AstraZeneca. SN and JJJvG are employees of AstraZeneca R\&D, Mölndal, Sweden. 


\section{References}

1. Cosemans JM, Munnix IC, Heller R, Jackson SP, Heemskerk JW. Continuous signaling via PI3K isoforms b and $g$ is required for platelet ADP receptor function in dynamic thrombus stabilization. Blood. 2006;108:30453052.

2. Cattaneo M. The platelet $\mathrm{P} 2 \mathrm{Y}_{12}$ receptor for adenosine diphosphate: congenital and drug-induced defects. Blood. 2011;117:2102-2112.

3. Swieringa F, Kuijpers MJ, Heemskerk JW, van der Meijden PE. Targeting platelet receptor function in thrombus formation: the risk of bleeding. Blood Rev. 2014;28:9-21.

4. Steg PG, James SK, Atar D, et al. ESC guidelines for the management of acute myocardial infarction in patients presenting with ST-segment elevation. Eur Heart J. 2012;33:2569-2619.

5. Wiviott SD, Braunwald $\mathrm{E}, \mathrm{McCabe} \mathrm{CH}$, et al. Prasugrel versus clopidogrel in patients with acute coronary syndromes. N Engl J Med. 2007;357(20):2001-2015.

6. Wallentin L, Becker RC, Budaj A, et al. Ticagrelor versus clopidogrel in patients with acute coronary syndromes. N Engl J Med. 2009;361:1045-1057.

7. Gurbel PA, Bliden KP, Butler K, et al. Randomized double-blind assessment of the onset and offset of the antiplatelet effects of ticagrelor versus clopidogrel in patients with stable coronary artery disease: the onset/ offset study. Circulation. 2009;120:2577-2585.

8. Cannon CP, Harrington RA, James $\mathrm{S}$, et al. Comparison of ticagrelor with clopidogrel in patients with a planned invasive strategy for acute coronary syndromes (PLATO): a randomised double-blind study. Lancet. 2010;375:283-293.

9. Price MJ, Walder JS, Baker BA, et al. Recovery of platelet function after discontinuation of prasugrel or clopidogrel maintenance dosing in aspirin-treated patients with stable coronary disease. J Am Coll Cardiol. 2012;59:2338-2343.

10. Floyd CN, Passacquale G, Ferro A. Comparative pharmacokinetics and pharmacodynamics of platelet adenosine diphosphate receptor antagonists and their clinical implications. Clin Pharmacokinet. 2012;51:429442.

11. Van Giezen JJJ, Humphries RG. Preclinical and clinical studies with selective reversible direct $P 2 Y_{12}$ antagonists. Sem Thromb Hemost. 2005;31:195-204.

12. Van Giezen JJJ, Nilsson L, Berntsson $P$, et al. Ticagrelor binds to human $P 2 Y_{12}$ independently from ADP but antagonizes ADP-induced receptor signaling and platelet aggregation. J Thromb Haemost. 2009;7:15561565.

13. Ho PM, Peterson ED, Wang L, et al. Incidence of death and acute myocardial infarction associated with stopping clopidogrel after acute coronary syndrome. JAMA. 2008;299:532-539.

14. Ho PM, Tsai TT, Wang TY, et al. Adverse events after stopping clopidogrel in post-acute coronary syndrome patients: insights from a large integrated healthcare delivery system. Circ Cardiovasc Qual Outcomes. 2010;3:303-308.

15. Mylotte D, Peace AJ, Tedesco AT, et al. Clopidogrel discontinuation and platelet reactivity following coronary stenting. J Thromb Haemost. 2011;9:24-32.

16. Charlot M, Nielsen LH, Lindhardsen J, et al. Clopidogrel discontinuation after myocardial infarction and risk of thrombosis: a nationwide cohort study. Eur Heart J. 2012;33:2527-2534.

17. Mauri L, Kereiakes DJ, Yeh RW, et al. Twelve or 30 months of dual antiplatelet therapy after drug-eluting stents. N Engl J Med. 2014;371(23):2155-2166.

18. Kuijpers MJ, Megens RT, Nikookhesal E, et al. Role of newly formed platelets in thrombus formation in rat after clopidogrel treatment: comparison to the reversible binding of $\mathrm{P}_{2} \mathrm{Y}_{12}$ antagonist ticagrelor. Thromb Haemost. 2011;106:1179-1188.

19. Ault KA, Knowles C. In vivo biotinylation demonstrates that reticulated platelets are the youngest platelets in circulation. Exp Hematol. 1995;23:996-1001.

20. Harrison P, Goodall AH. "Message in the platelet"- more than just vestigial mRNA! Platelets. 2008;19:395404.

21. Roth GJ, Hickey MJ, Chung DW, Hickstein DD. Circulating human blood platelets retain appreciable amounts of poly (A)+ RNA. Biochem Biophys Res Commun. 1989;160:705-710

22. Robinson M, Machin S, Mackie I, Harrison P. In vivo biotylation studies: specificity of labelling of reticulated platelets by thiazole orange and mepacrine. Br J Haematol. 2000;108:859-864. 
23. Rinder HM, Tracey JB, Recht $M$, et al. Differences in platelet a-granule release between normals and immune thrombocytopenic patients and between young and old platelets. Thromb Haemost. 1998;80:457462.

24. Karpatkin S. The heterogeneity of human platelets. Functional evidence suggestive of young and old platelets. J Clin Invest. 1969;48:1083-1087.

25. Guthikonda S, Alviar CL, Vaduganathan $\mathrm{M}$, et al. Role of reticulated platelets and platelet size heterogeneity on platelet activity after dual antiplatelet therapy with aspirin and clopidogrel in patients with stable coronary artery disease. J Am Coll Cardiol. 2008;52:743-749.

26. Perl L, Lerman-Shivek H, Rechavia E, et al. Response to prasugrel and levels of circulating reticulated platelets in patients with ST-segment elevation myocardial infarction. J Am Coll Cardiol 2014;63:513-517.

27. Ibrahim H, Nadipalli S, DeLao T, Guthikonda S, Kleiman NS. Immature platelet fraction (IPF) determined with an automated method predicts clopidogrel hyporesponsiveness. J Thromb Thrombolysis. 2012;33:137142.

28. Van Gorp RM, Feijge MA, Vuist WM, Rook MB, Heemskerk JW. Irregular spiking in free calcium concentration in human platelets. Regulation by modulation of the inositol trisphosphate receptors. Eur $\mathrm{J}$ Biochem. 2002;269:1543-1552.

29. Schols SE, Feijge MA, Lance MD, et al. Effects of plasma dilution on tissue factor-induced thrombin generation and thromboelastography: partly compensating role of platelets. Transfusion. 2008;48:23842394.

30. Ninivaggi M, Feijge MA, Baaten CC, et al. Additive roles of platelets and fibrinogen in whole-blood fibrin clot formation upon dilution as assessed by thromboelastometry. Thromb Haemost. 2014;111:447-457.

31. Van der Meijden PE, Bouman AC, Feijge MA, et al. Platelet dysfunction in thrombosis patients treated with vitamin $\mathrm{K}$ antagonists and recurrent bleeding. Plos One. 2013;8:e64112.

32. Velik-Salchner C, Maier S, Innerhofer P, et al. Point-of-care whole blood impedance aggregometry versus classical light transmission aggregometry for detecting aspirin and clopidogrel: the results of a pilot study. Anesth Analg. 2008;107:1798-1806.

33. Alexopoulos D, Galati A, Xanthopoulou I, et al. Ticagrelor versus prasugrel in acute coronary syndrome patients with high on-clopidogrel platelet reactivity following percutaneous coronary intervention: a pharmacodynamic study. J Am Coll Cardiol. 2012;60(3):193-199.

34. McCabe DJ, Harrison P, Sidhu PS, Brown MM, Machin SJ. Circulating reticulated platelets in the early and late phases after ischaemic stroke and transient ischaemic attack. Br J Haematol. 2004;126:861-869.

35. De Witt SM, Swieringa F, Cavill R, et al. Identification of platelet function defects by multiparameter assessment of thrombus formation. Nat Commun. 2014;16:e4257.

36. Nergiz-Unal R, Cosemans JM, Feijge MA, et al. Stabilizing role of platelet $P 2 Y_{12}$ receptors in shear dependent thrombus formation on ruptured plaques. Plos One. 2010;5:e10130.

37. Frelinger AL, Barnard MR, Fox ML, Michelson AD. The platelet activity after clopidogrel termination (PACT) study. Circulation. 2010;3:442-449.

38. Farid NA, Kurihara A, Wrighton SA. Metabolism and disposition of the thienopyridine antiplatelet drugs ticlopidine, clopidogrel and prasugrel in humans. J Clin Pharmacol. 2010;50:126-142.

39. Hagihara K, Kurihara A, Kawai K, et al. Absorption, distribution and excretion of the new thienopyridine agent prasugrel in rats. Xenobiotica. 2007;37:788-801.

40. Cosemans JM, van Kruchten R, Olieslagers S, et al. Potentiating role of Gas6 and Tyro3, Axl and Mer (TAM) receptors in human and murine platelet activation and thrombus stabilization. J Thromb Haemost. 2010;8:1797-1808.

41. McBane RD, Gonzalez C, Hodge DO, Wysokinski WE. Propensity for young reticulated platelet recruitment into arterial thrombi. J Thromb Thrombolysis. 2014;37:148-154.

42. Sibbing D, Stegherr J, Braun S, et al. A double-blind, randomized study on prevention and existence of a rebound phenomenon of platelets after cessation of clopidogrel treatment. J Am Coll Cardiol. 2010;55:558565.

43. Fiedler KA, Mehilli J, Kufner S, et al. Randomized, double-blind trial on the value of tapered discontinuation of clopidogrel maintenance therapy after drug-eluting stent implantation. Intracoronary stenting and antithrombotic regimen: caution in discontinuing clopidogrel therapy. Thromb Haemost. 2014;111:10411049.

44. Jakubowski JA, Li YG, Payne CD, Small DS, Winters KJ. Absence of "rebound" platelet hyperreactivity following cessation of prasugrel. Thromb Haemost. 2011;106:174-176. 
45. Yedidya I, Netzer A, Vaduganathan M, Solodky A, Kornowski R, Lev El. Clopidogrel tapering as a strategy to attenuate platelet rebound phenomenon in patients with bare-metal stents. J Thromb Thrombolysis. 2012;33:16-21.

46. Zafar MU, Santos-Gallego DA, Vorchheimer DA, et al. Platelet function normalization after a prasugrel loading dose: time-dependent effect of platelet supplementation. J Thromb Haemost. 2013;11:100-106. 


\section{Supplemental data}

\section{Supplemental methods}

\section{Materials}

2MeS-ADP and D-Phe-Pro-Arg chloromethyl ketone (PPACK) were from SantaCruz Biotechnology (Santa Cruz, CA, USA); fluorescein isothiocyanate (FITC)-conjugated PAC1 antibody against active integrin $\alpha_{l \mid b} \beta_{3}$ and thiazole orange from Becton-Dickinson Bioscience (Franklin Lakes NJ, USA), and aspirin was from Sanofi (Paris, France). Ticagrelor and the clopidogrel active metabolite (CAM) were kindly provided by AstraZeneca R\&D (Mölndal, Sweden). 5'-Cy5-conjugated oligo-dA20 and oligo-dT20 were obtained from Eurogentec (Maastricht, The Netherlands). Alexa Fluor (AF)-647 and Oregon Green (OG)488 conjugated human fibrinogen were from Invitrogen (Bleiswijk, The Netherlands). Collagen type I came from Nycomed Pharma (Munich, Germany); 3,3'-dihexyloxa carbocyanine iodide $\left(\mathrm{DiOC}_{6}\right.$ ) from Anaspec (Fremont CA, USA); CellVue Maroon from eBioscience (San Diego CA, USA), and iloprost from Bayer Pharma (Berlin, Germany). Multiplate test kits were from Roche Diagnostics (Basel, Switzerland). Other compounds came from Sigma (St. Louis, MO, USA).

\section{Patients and control subjects}

This study was approved by the local medical ethics committee (MEC 12-3-075). All patients and healthy volunteers gave written informed consent for participation according to the Helsinki declaration. Sixteen patients were studied who were treated with prasugrel (10 mg/day) for one year and long-term aspirin (80-100 mg/day) due to a myocardial infarction with ST elevation. After one year of prasugrel treatment, blood was collected on the last day of prasugrel intake, and at 1, 2, 5 and 30 days after the last dose. From two patients, blood samples were also collected after 12 days to better understand the delayed regain of platelet function. Patients with a malignancy, active infection or a known platelet disorder were not included. Blood was obtained by venipuncture with a Vacutainer 21-gauge needle (Becton-Dickinson Bioscience). Blood collection was into Vacuette tubes, containing $\mathrm{K}_{2}$-EDTA, for measurement of hemostatic variables and immature platelet fraction using a Sysmex XN-9000 analyzer, according to protocols of the supplier (Sysmex, Chuo-ku Kobe, Japan). Blood samples were also collected into $3.2 \%(\mathrm{w} / \mathrm{v})$ trisodium citrate for platelet function measurements, and into hirudin for whole-blood platelet aggregation. Control experiments were performed with blood drawn from healthy volunteers. Collection was into trisodium citrate, or into acidic citrate dextrose (ACD, $80 \mathrm{mM}$ trisodium citrate, $52 \mathrm{mM}$ citric acid and $180 \mathrm{mM}$ glucose) for the preparation of washed platelets. ${ }^{1}$ 
Preparation of platelet-rich plasma, platelets and red cells

Platelet-rich plasma (PRP) was prepared by centrifuging citrate-anticoagulated blood at $240 \mathrm{~g}$ for 15 minutes at room temperature. Platelet-free plasma was obtained by centrifuging citrate-anticoagulated blood twice at $2630 \mathrm{~g}$ for 10 minutes. Washed platelets were prepared from ACD-anticoagulated blood, as described ${ }^{2}$, and were suspended in Hepes buffer pH $7.45(10 \mathrm{mM}$ Hepes, $136 \mathrm{mM} \mathrm{NaCl}, 2.7 \mathrm{mM} \mathrm{KCl}, 2 \mathrm{mM}$ $\mathrm{MgCl}_{2}, 0.1 \%$ glucose and $0.1 \%$ bovine serum albumin). Platelet counts were determined with a thrombocounter XP300 Sysmex analyzer (Sysmex, Chuo-ku Kobe, Japan).

Washed red blood cells were prepared by centrifuging citrate-anticoagulated blood at $240 \mathrm{~g}$ for 15 minutes at room temperature. ${ }^{3}$ After removal of PRP, Hepes buffer $\mathrm{pH} 7.45$ was added in a 1:2 volume ratio. The red blood cells were centrifuged twice at $2630 \mathrm{~g}$ for 10 minutes, with resuspension in Hepes buffer $\mathrm{pH}$ 7.45. This resulted in a preparation of $>99.9 \%$ red cells.

\section{Irreversible P2Y12 inhibition in vitro}

PRP from healthy donors was treated with $100 \mu \mathrm{M}$ lysine aspirin for 30 minutes, and platelets were pelleted by centrifugation. ${ }^{1}$ The resuspended cells were incubated for 15 minutes with $10 \mu \mathrm{M}$ CAM or vehicle medium. Residual unbound CAM was removed by a second final centrifugation step, where $5 \mathrm{nM}$ iloprost was added to prevent platelet activation. Mixtures of the washed CAM-treated and vehicle-treated platelets in Hepes buffer $\mathrm{pH} 7.45$ were used for measurement of: platelet aggregation (light transmission aggregometry in the presence of $2 \mathrm{mM} \mathrm{CaCl}$ and $0.1 \mathrm{mg} / \mathrm{ml}$ fibrinogen); integrin $\alpha_{11 \mathrm{~B}} \beta_{3}$ activation by flow cytometry in the presence of $75 \mu \mathrm{g} / \mathrm{ml} \mathrm{OG488-fibrinogen;} \mathrm{and}$ perfusion experiments with reconstituted whole blood.

\section{Platelet aggregation}

Aggregation of platelets in PRP was measured using a Chronolog aggregometer (Stago, Asnières sur Seine Cedex, France). ${ }^{4}$ Preincubation with ticagrelor or vehicle medium was for 5 minutes at $37^{\circ} \mathrm{C}$. Aggregation of platelets in whole blood was measured by Multiplate impedance aggregometry (Roche Diagnostics, Basel, Switzerland) as described. ${ }^{5}$ Aggregation was measured in response to ADP $(6.4 \mu \mathrm{M})$, arachidonic acid $(0.5 \mathrm{mM})$, collagen $(3.2 \mu \mathrm{g} / \mathrm{ml})$ or thrombin receptor-activating peptide SFLLRN (TRAP, $32 \mu \mathrm{M})$ at $37^{\circ} \mathrm{C}$ during 6 minutes. Ticagrelor $(1 \mu \mathrm{M})$ was added in vitro to block residual P2 $\mathrm{Y}_{12}$ activity, where indicated. Extent of platelet aggregation was assessed from the area under the impedance curve.

\section{Flow cytometric analysis of platelet subpopulations}

Flow cytometric measurements were performed on an Accuri 66 flow cytometer with CFlow Plus software (Becton-Dickinson Bioscience). To check for integrin $\alpha_{116} \beta_{3}$ activation, samples of citrated patient blood were diluted (1:20) into Hepes buffer $\mathrm{pH} 7.45$, and incubated with $1 \mu \mathrm{M}$ ticagrelor or vehicle control for 15 minutes at room temperature. 
Platelets were activated with $1 \mu \mathrm{M}$ 2MeS-ADP in the presence of FITC-conjugated PAC-1 antibody $(1.25 \mu \mathrm{g} / \mathrm{ml})$ against the activated $\alpha_{11 \mathrm{~b}} \beta_{3}$ integrin for 10 minutes. Ticagrelor (1 $\mu \mathrm{M}$ ) was added, where indicated. Activated platelets were identified as before. ${ }^{4}$

Juvenile platelets were identified using two different methods of mRNA staining, i.e. with thiazole orange ${ }^{6}$ or by a novel method using Cy5-labelled oligo-dT, which binds to the poly-A tail of mRNA species. Thiazole orange (15\% in filtered PBS: $136 \mathrm{mM} \mathrm{NaCl}, 2.7$ $\mathrm{mM} \mathrm{KCl}, 1.47 \mathrm{mM} \mathrm{KH}_{2} \mathrm{PO}_{4}, 6.46 \mathrm{mM} \mathrm{Na}_{2} \mathrm{HPO}_{4}$ ) was added to PRP for 30 minutes at room temperature, according to established procedures. ${ }^{6}$ Platelets were then centrifuged at $5550 \mathrm{~g}$ for 3 minutes to remove excess and unbound thiazole orange, and resuspended in Hepes buffer $\mathrm{pH}$ 7.45. Samples were activated with $1 \mu \mathrm{M}$ 2MeS-ADP in the presence of AF647-fibrinogen ( $75 \mathrm{\mu g} / \mathrm{ml})$ for 15 minutes. For staining with 5'Cy5-oligo-dT, washed platelets in suspension $\left(1 \times 10^{8} / \mathrm{ml}\right)$ were activated with $1 \mu \mathrm{M} 2 \mathrm{MeS}$-ADP in the presence of OG488-fibrinogen $(75 \mu \mathrm{g} / \mathrm{ml})$ for 15 minutes. Samples were fixed (15 minutes) with $0.2 \%$ formaldehyde and permeabilized ( 10 minutes) with $0.1 \%$ saponin. 5 '-Cy5-oligo-dT $(1 \mu \mathrm{M})$ was incubated for 15 minutes at $37^{\circ} \mathrm{C}$. For all samples, $5^{\prime} \mathrm{Cy}$-oligo-dA $(1 \mu \mathrm{M})$ was used as a negative control probe to check for specificity of the staining with $5^{\prime} \mathrm{Cy} 5$-oligo$\mathrm{dT}$. Color compensation was not required as fluorescent spectra did not overlap.

The average percentage of juvenile platelets as analyzed by the thiazole orange staining and the oligo-dT staining was $6.7 \%( \pm 1.9 \%)$ and $21.5 \%( \pm 5.8 \%)$ respectively. The discrepancy in the percentage of detected juvenile platelets can be explained by the higher sensitivity of the oligo-dT staining to detect mRNA in comparison to thiazole orange. In order to use a uniform definition of juvenile platelets, the threshold for juvenile platelets was based on the IPF as determined by the Sysmex XN9000 analyzer, which is an internationally validated method in the clinic. An alternative analysis of juvenile platelets, based on the negative controls of both stainings, is presented in the supplements (Supplemental Fig.3).

\section{Thrombus formation in whole blood}

Whole-blood thrombus formation on microspots in a parallel-plate flow chamber was measured, basically as described before. ${ }^{7}$ Briefly, $0.5 \mu \mathrm{l}$ microspots containing either collagen I $(100 \mu \mathrm{g} / \mathrm{ml})$ or fibrinogen $(250 \mu \mathrm{g} / \mathrm{ml})$ plus vWF $(50 \mu \mathrm{g} / \mathrm{ml})$ were perfused with citrate-anticoagulated whole blood, which was recalcified with $7.5 \mathrm{mM} \mathrm{CaCl}_{2}$ and 3.75 $\mathrm{mM} \mathrm{MgCl} 2$ in the presence of $40 \mu \mathrm{M}$ PPACK immediately before the experiment. Patient blood samples were perfused through the chamber for 4 minutes at a wall-shear rate of $1600 \mathrm{~s}^{-1}$, while $2 \mathrm{MeS}-\mathrm{ADP}(0.1 \mu \mathrm{M}$, f.c.) was co-perfused with a second pump. Ticagrelor was added where indicated. Thrombi were stained with AF647-labelled fibrinogen and, when indicated, $\mathrm{DiOC}_{6}$, as described elsewhere. ${ }^{7}$ Brightfield and fluorescence microscopic images were captured with an EVOS fluorescence microscope, equipped with a 60x oil objective. Images were analysed using Metamorph (Molecular Devices, Sunnyvale CA, USA) and ImageJ (open access) software. ${ }^{7}$

For measurement of thrombus formation of reconstituted blood samples from healthy controls, mixtures of CAM- and vehicle-treated platelets $\left(2.5 \times 10^{8} / \mathrm{ml}\right.$, final count $)$ were added to washed red cells ( $45 \%$ hematocrit) and plasma (30-35\% of total volume). In these experiments, the CAM-treated platelets were pre-labeled with the membrane 
probe CellVue Maroon (1.6 $\mu \mathrm{M})$, whereas the vehicle-treated platelets were pre-labeled with the membrane probe PKH26 $(0.8 \mu \mathrm{M})$. Both probes were not transferrable from cell to cell (data not shown). The reconstituted whole blood was again co-perfused with 2MeS-ADP over collagen. Microscopic DIC and confocal fluorescent images were taken using a Zeiss LSM7 microscope (Oberkochen, Germany). ${ }^{7}$

\section{Statistical analysis}

Statistical analysis was performed using the SPSS Statistics 22 package (Armonk, NY, USA). Statistical analysis was performed using a one-way-repeated-measures-ANOVA or with a Friedman test with a post hoc Wilcoxon signed rank test. Bonferroni correction was applied when comparing multiple groups. 
Supplemental Table 1. Study characteristics. Means \pm SD.

\begin{tabular}{|c|c|}
\hline Variables & \\
\hline \multicolumn{2}{|l|}{ Patient characteristics } \\
\hline Age (years) & $59 \pm 9$ \\
\hline Female/male (n) & $3 / 13$ \\
\hline Diabetes Mellitus (n) & 3 \\
\hline Aspirin use (n) & 16 \\
\hline Statins use (n) & 13 \\
\hline \multicolumn{2}{|l|}{ Hematological variables } \\
\hline Hematocrit (L/L) & $0.435 \pm 0.035$ \\
\hline Platelet count $\left(10^{9} / \mathrm{L}\right)$ & $239 \pm 81$ \\
\hline Mean platelet volume (fL) & $10.8 \pm 0.7$ \\
\hline Immature platelet fraction (IPF) Sysmex (\%) & $3.6 \pm 1.9$ \\
\hline
\end{tabular}
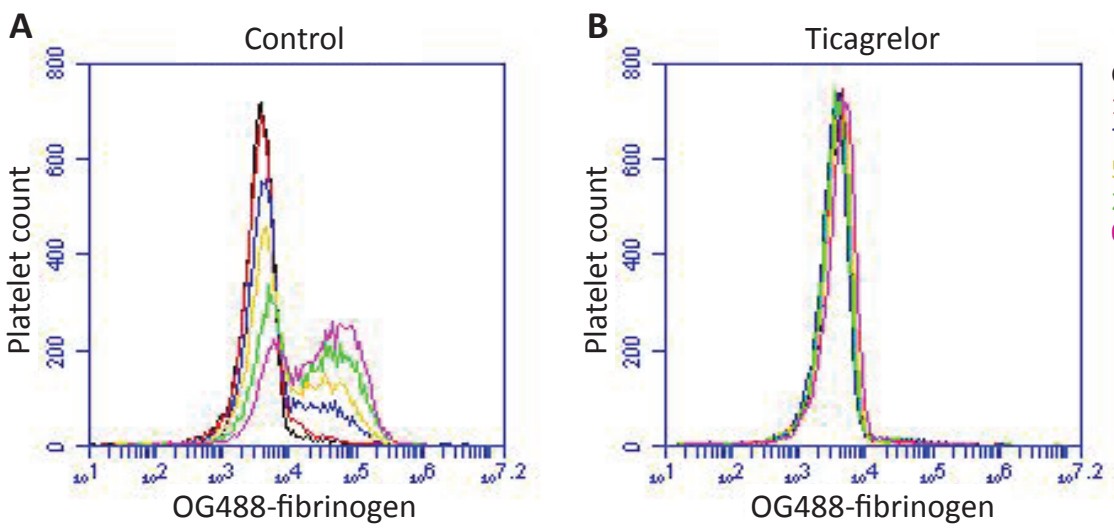

Control, no ADP

$100 \%$ CAM + ADP

$75 \%$ CAM + ADP

$50 \%$ CAM + ADP

$25 \%$ CAM + ADP

$0 \% C A M+A D P$

Supplemental Figure 1. Impaired fibrinogen binding of populations of $P 2 Y_{12}$-inhibited platelets. Mixtures of control platelets and CAM-treated platelets were preincubated with $1 \mu \mathrm{M}$ ticagrelor or vehicle, and stimulated for 15 minutes with $20 \mu \mathrm{M}$ 2MeS-ADP in the presence of OG488-fibrinogen. Fibrinogen binding was assessed by flow cytometry. Shown are representative histograms of fibrinogen binding after stimulation in the presence of vehicle (A) or ticagrelor (B). 

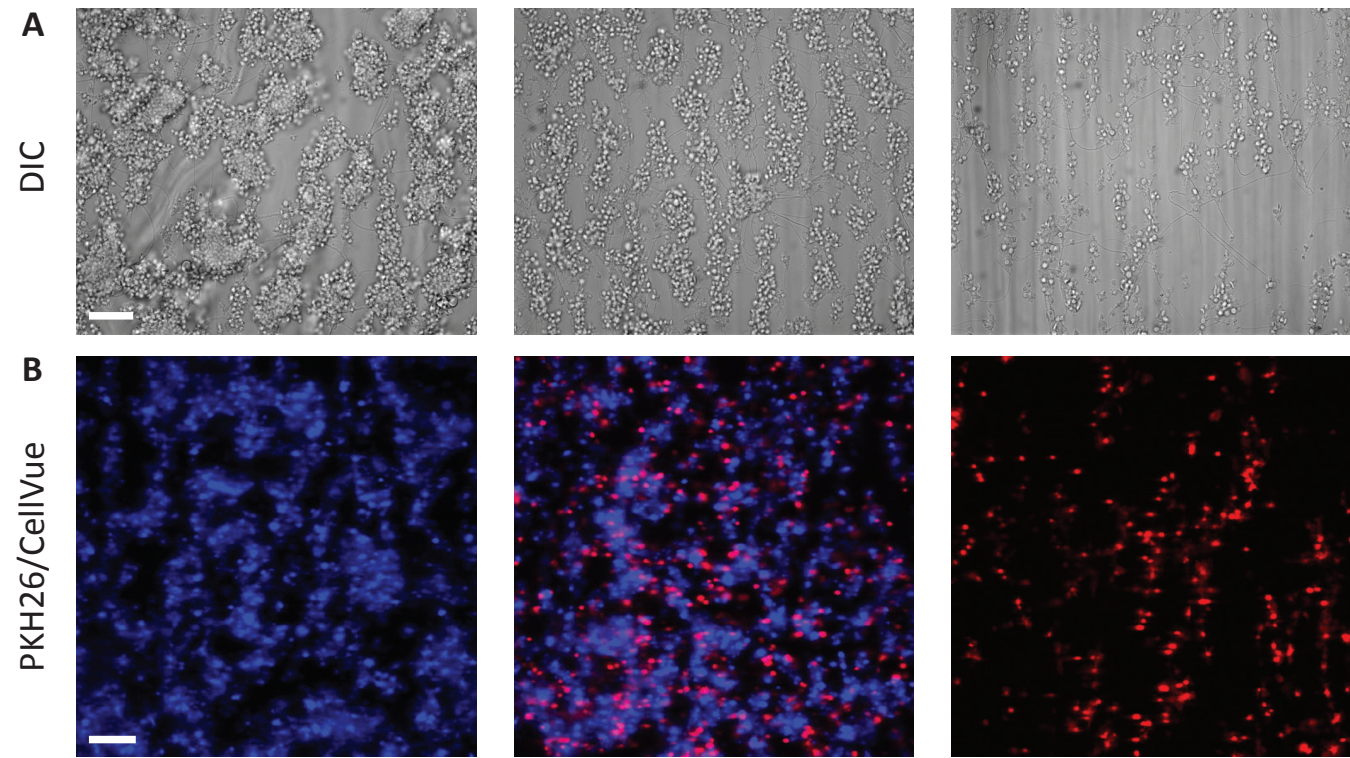

0

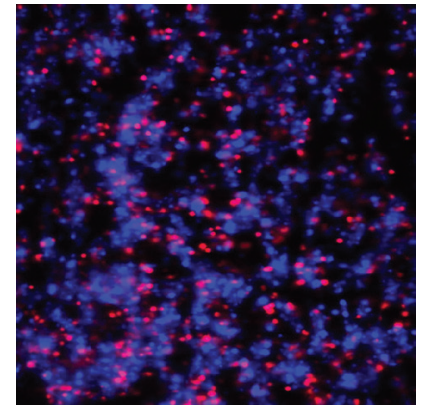

50

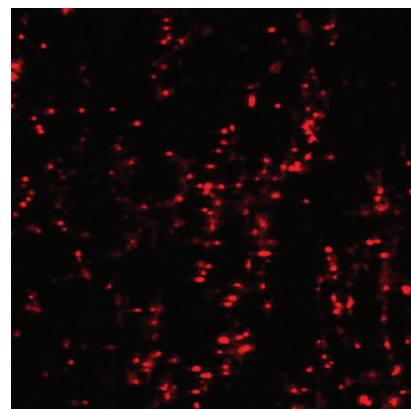

100

$\%$ CAM treated platelets

Supplemental Figure 2. Impaired contribution to thrombus formation of $\mathrm{P}_{2} \mathrm{Y}_{12}$-inhibited platelets. Reconstituted blood with different fractions of CAM-treated platelets was perfused 4 minutes over collagen at $1600 \mathrm{~s}^{-1}$ in the presence of 2MeS-ADP. Uninhibited platelets were prelabeled with PKH26 (blue) and CAMtreated platelets with CellVue Maroon (red). Shown are representative DIC (A) and dual-color fluorescence (B) images. Bars = $25 \mu \mathrm{m}$. See further, Figure 1. 
A Oligo-dT staining, unstimulated
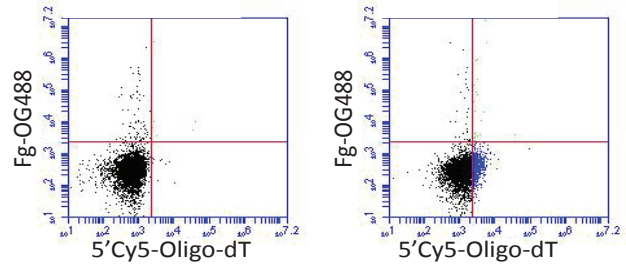

B Oligo-dT staining, ADP activated
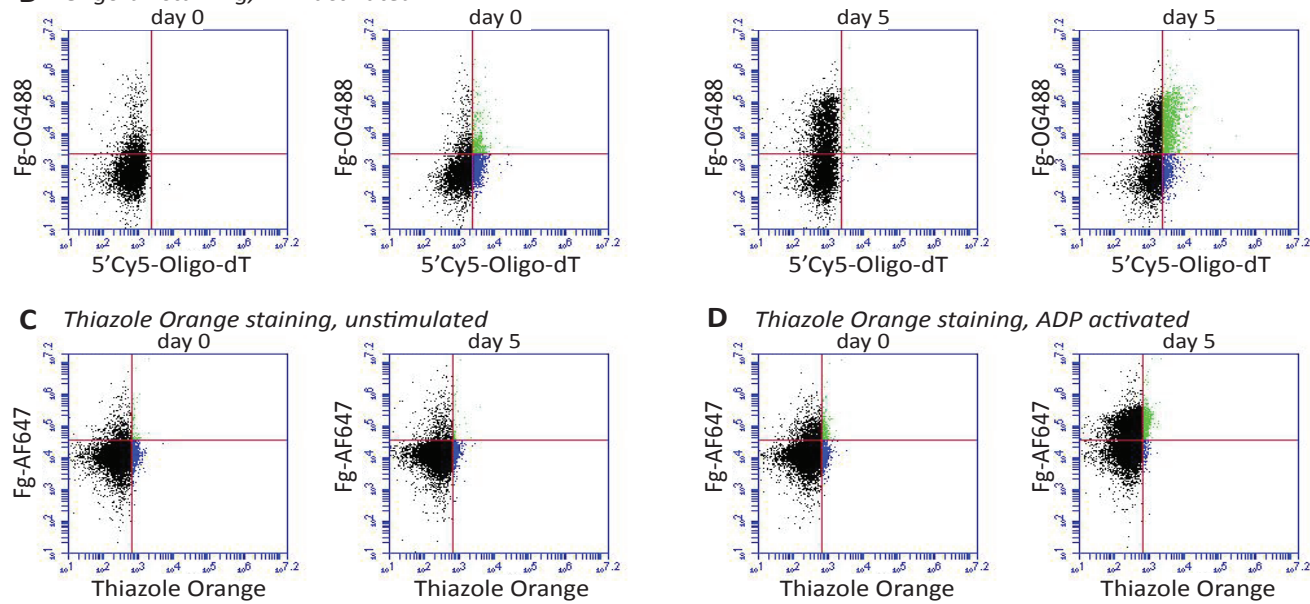

\begin{tabular}{|l|l|l|l|l|l|l|}
\hline \multicolumn{2}{|l|}{ Activation ratio } & day 0 & day 1 & day 2 & day $\mathbf{5}$ & day 30 \\
\hline $\begin{array}{l}\text { Oligo-dT } \\
\text { staining }\end{array}$ & Mature & $0.17( \pm 0.03)$ & $0.24( \pm 0.03)$ & $0.33( \pm 0.03)$ & $0.82( \pm 0.10)$ & $2.70( \pm 0.26)$ \\
\cline { 2 - 7 } & Juvenile & $0.44( \pm 0.08)^{*}$ & $0.66( \pm 0.11)^{*}$ & $\begin{array}{l}0.84 \\
( \pm 0.09)^{* * *}\end{array}$ & $\begin{array}{l}1.81 \\
( \pm 0.22)^{* *}\end{array}$ & $6.30( \pm 0.99)^{*}$ \\
\hline $\begin{array}{l}\text { Thiazole } \\
\text { Orange }\end{array}$ & Mature & $0.18( \pm 0.04)$ & $0.24( \pm 0.04)$ & $0.41( \pm 0.06)$ & $1.02( \pm 0.12)$ & $3.35( \pm 0.59)$ \\
\cline { 2 - 7 } & Juvenile & $0.38( \pm 0.08)^{*}$ & $\begin{array}{l}0.50 \\
( \pm 0.08)^{* * *}\end{array}$ & $\begin{array}{l}0.92 \\
( \pm 0.13)^{* * *}\end{array}$ & $\begin{array}{l}2.42 \\
( \pm 0.27)^{* * *}\end{array}$ & $\begin{array}{l}8.18 \\
( \pm 1.32)^{* *}\end{array}$ \\
\hline
\end{tabular}

Supplemental Figure 3. Alternative analysis confirms higher reactivity of juvenile platelets and increased activation in time upon prasugrel offset Juvenile platelets were detected using the oligo-dT staining or the thiazole orange staining, as described for Figure 5. Shown are representative dot plots of oligo-dA/dT or thiazole orange vs. Fg-OG488/AF647. Thresholds for juvenile and mature platelets were based on the negative controls oligo-dA and PBS for the oligo-dT and thiazole orange staining respectively. Thresholds for activated platelets were based on unstimulated samples. Unstimulated juvenile platelets (blue) and activated juvenile platelets (green) are depicted. Representative dot plots of unstimulated (A) or ADP-activated (B) mature and juvenile platelets as identified by the oligo-dT staining. Representative dot plots of unstimulated (C) or ADP-activated (D) mature and juvenile platelets using the thiazole orange staining.

Table. Using the percentage of platelets in each quadrant of the dot plots, the activation ratio was calculated for both mature (Q-UL/Q-LL) and juvenile platelets (Q-UR/Q-LR) using either staining. Mean \pm SEM ( $n=16)$, $* p<0.05, * * p<0.01, * * * p<0.001$ vs. corresponding mature ratio (ANOVA with Bonferroni correction). 
A
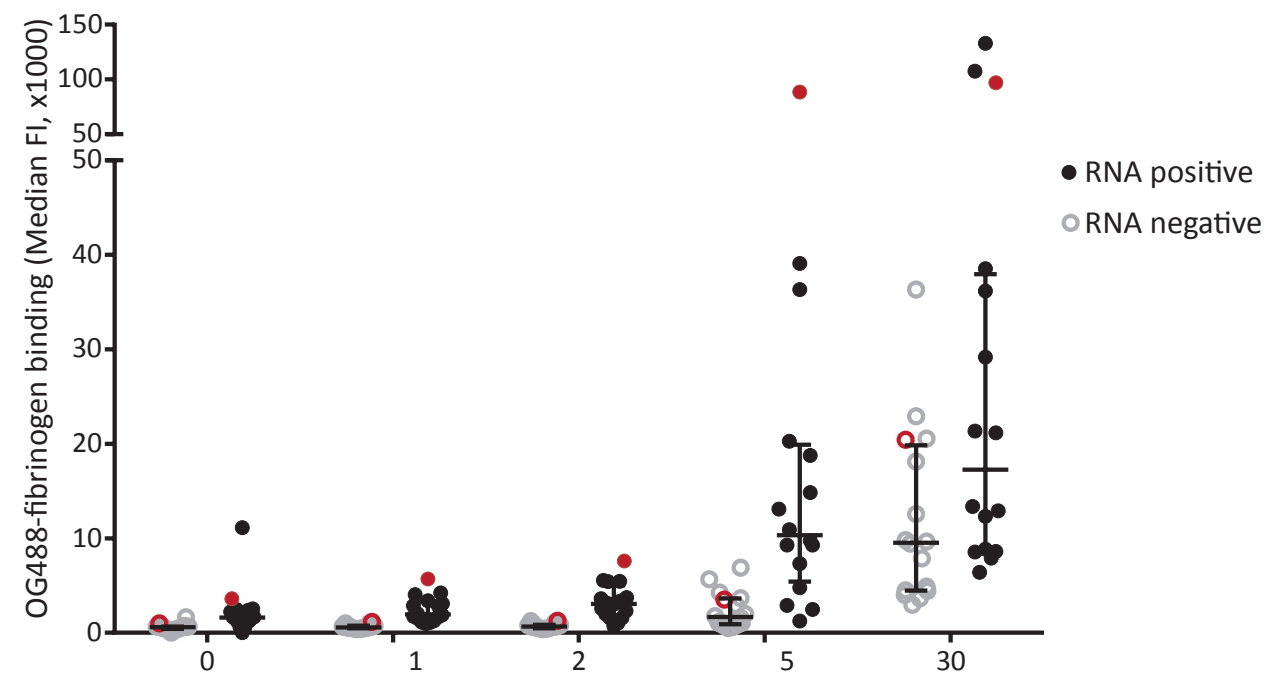

Days offset

B

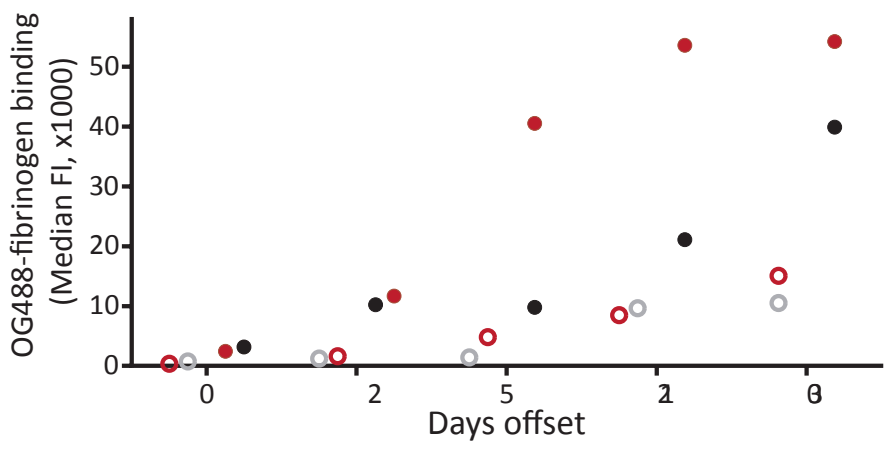

- RNA positive

- RNA negative

Supplemental Figure 4. Increased ADP-induced $\alpha_{111} \beta_{3}$ activation of juvenile platelets formed upon prasugrel offset. Platelets from patients during offset from prasugrel were activated and analyzed by flow cytometry. Juvenile platelets were identified by staining with the mRNA probe, $5^{\prime} \mathrm{Cy} 5$-oligo-dT. See further Figure 5. Washed platelets were activated with $1 \mu \mathrm{M} 2 \mathrm{MeS}$-ADP in the presence of OG488-fibrinogen. The cells were fixed and permeabilized with saponin to allow staining of mRNA by incubation with 5'Cy5-oligo-dT. Shown is extent of $\alpha_{11 b} \beta_{3}$ activation of mRNA-positive and -negative platelets, per subject. (A) Data from 16 patients, medians \pm IQR. (B) Data from additional patients including day 12 time points. Red dots are from patients with a high immature platelet fraction (IPF $\geq 7.0 \%$ ). 
A

VWF/fibrinogen

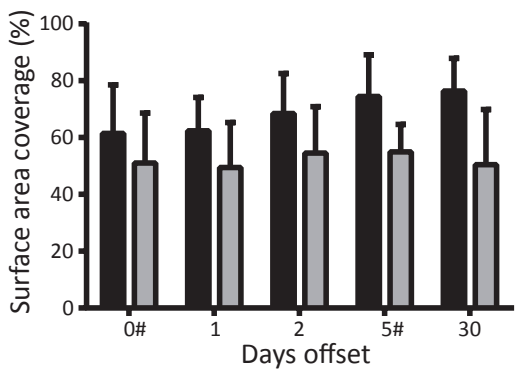

B

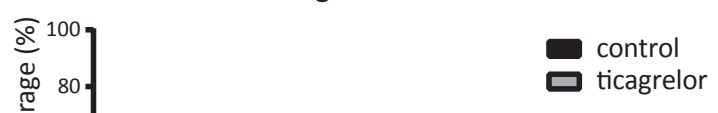

Supplemental Figure 5. Unchanged platelet adhesion in thrombus formation under flow upon prasugrel offset. Whole blood samples from patients (at indicated days after stopping prasugrel intake) were coperfused with 2MeS-ADP over microspots containing vWF/fibrinogen (A) or collagen type I (B), as in Figure 6. Brightfield images were analyzed for surface area coverage of all platelets (single platelets and aggregates) at different offset days. Means $\pm S D(n=16), * * * p<0.001$ vs. day 0 (ANOVA with Bonferroni correction); \#n $=15$. 


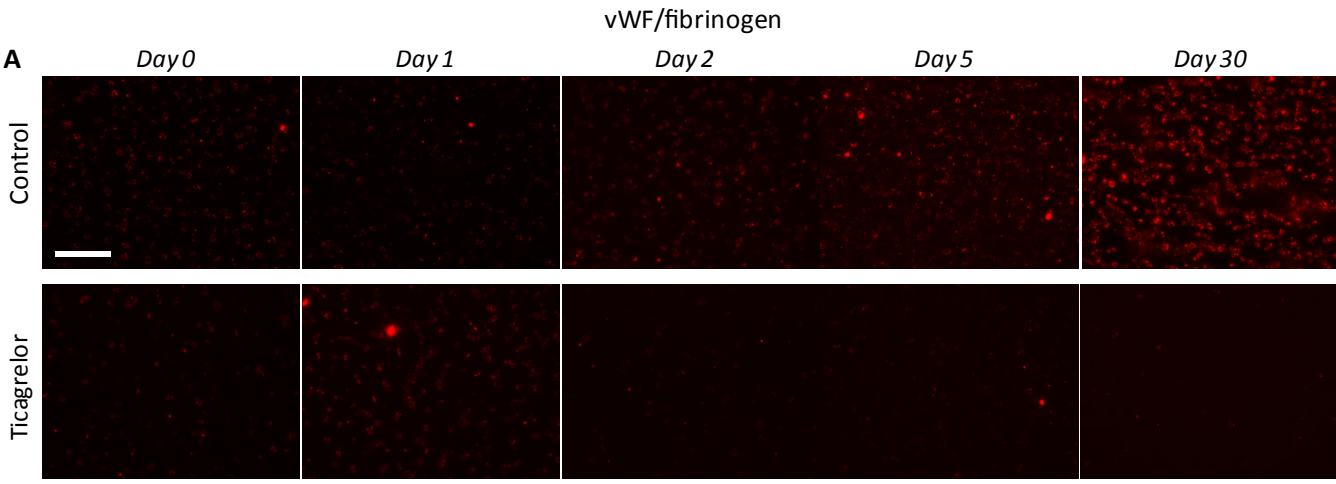

Collagen I
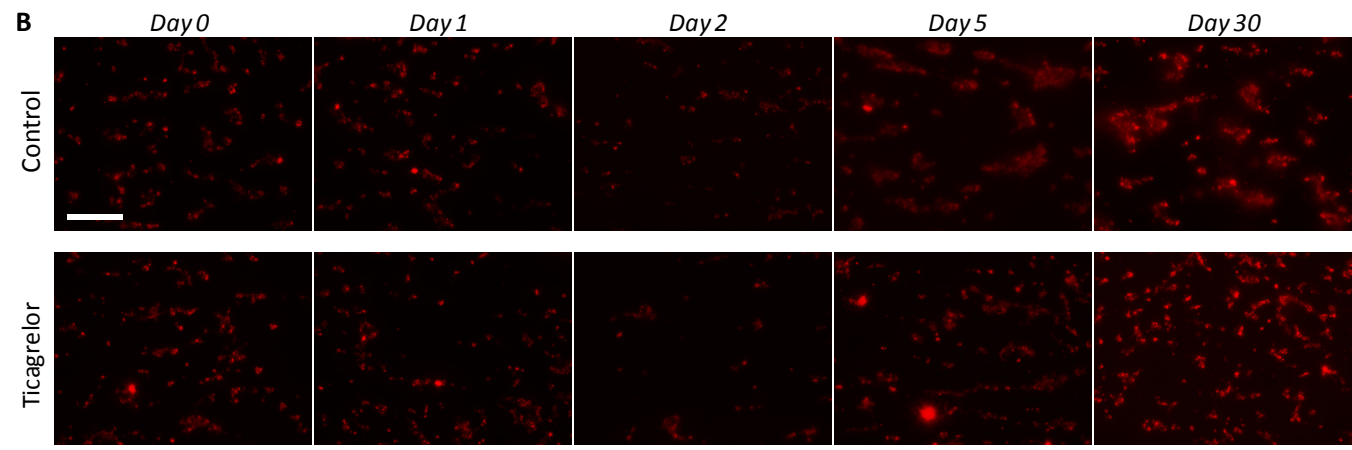

C
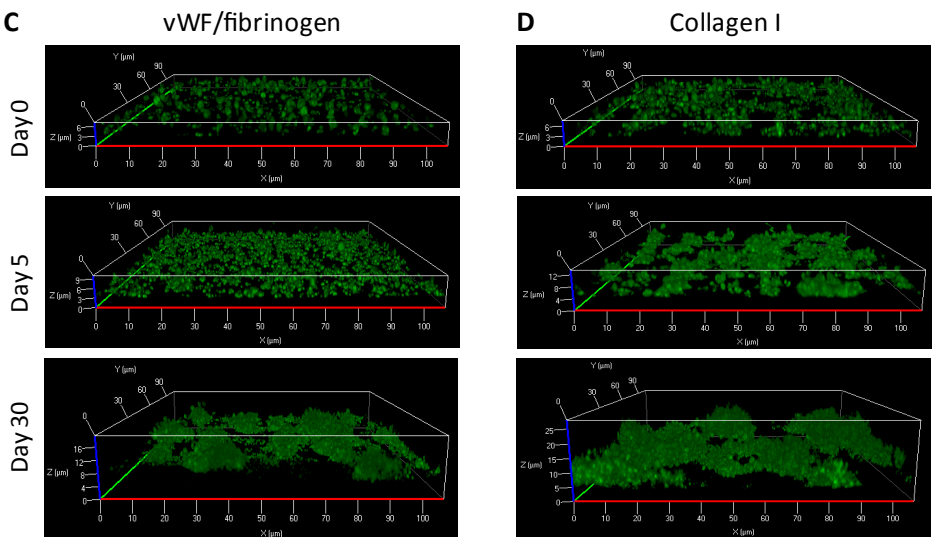

Supplemental Figure 6. Restored thrombus formation under flow upon prasugrel offset. Whole blood samples from patients (at indicated days after stopping prasugrel intake) were co-perfused with 2MeS-ADP over microspots containing vWF/fibrinogen or collagen type I, at a shear rate of $1600 \mathrm{~s}^{-1}$ for 4 minutes. See also Figure 6. Staining of thrombi was with AF647-fibrinogen and the platelet membrane label DiOC. Vehicle (control) or $10 \mu \mathrm{M}$ ticagrelor was added to the blood. (A, B) Shown are (for a representative patient) fluorescence images of fibrinogen binding (red) of the thrombi formed on vWF/fibrinogen or collagen I. Bars $=25 \mu \mathrm{m}$. (C, D) Representative confocal z-stacks of thrombi on vWF/fibrinogen or collagen I stained with $\mathrm{DiOC}_{6}$ (green). 


\section{References}

1. Van Gorp RM, Feijge MA, Vuist WM, Rook MB, Heemskerk JW. Irregular spiking in free calcium concentration in human platelets. Regulation by modulation of the inositol trisphosphate receptors. Eur J Biochem. 2002;269:1543-1552.

2. Schols SE, Feijge MA, Lance MD, et al. Effects of plasma dilution on tissue factor-induced thrombin generation and thromboelastography: partly compensating role of platelets. Transfusion. 2008;48:23842394.

3. Ninivaggi M, Feijge MA, Baaten CC, et al. Additive roles of platelets and fibrinogen in whole-blood fibrin clot formation upon dilution as assessed by thromboelastometry. Thromb Haemost. 2014;111:447-457.

4. Van der Meijden PE, Bouman AC, Feijge MA, et al. Platelet dysfunction in thrombosis patients treated with vitamin $\mathrm{K}$ antagonists and recurrent bleeding. Plos One. 2013;8:e64112.

5. Velik-Salchner C, Maier S, Innerhofer P, et al. Point-of-care whole blood impedance aggregometry versus classical light transmission aggregometry for detecting aspirin and clopidogrel: the results of a pilot study. Anesth Analg. 2008;107:1798-1806.

6. McCabe DJ, Harrison P, Sidhu PS, Brown MM, Machin SJ. Circulating reticulated platelets in the early and late phases after ischaemic stroke and transient ischaemic attack. Br J Haematol. 2004;126:861-869.

7. De Witt SM, Swieringa F, Cavill R, et al. Identification of platelet function defects by multiparameter assessment of thrombus formation. Nat Commun. 2014;5:4257. 



\section{Chapter 4}

Platelet control of fibrin distribution and microelasticity in thrombus formation under flow

Swieringa F, Baaten $\mathrm{CC}^{*}$, Verdoold $\mathrm{R}^{*}$, Mastenbroek TG, Rijnveld N, Van der Laan KO, Breel EJ,

Collins PW, Lancé MD, Henskens YM, Cosemans JM, Heemskerk JW, van der Meijden PE (*equal contribution)

Arterioscler Thromb Vasc Biol. 2016; 36 (4): 692-699 Reprinted with permission 


\section{Abstract}

Platelet- and fibrin-dependent thrombus formation is regulated by blood flow and exposure of collagen and tissue factor. However, interactions between these blood-borne and vascular components are not well understood. Here, we developed a method to assess whole blood thrombus formation on microspots with defined amounts of collagen and tissue factor, allowing determination of the mechanical properties and intrathrombus composition. Confining the collagen content resulted in diminished platelet deposition and fibrin formation at high shear flow conditions, but this effect was compensated by a larger thrombus size and increased accumulation of fibrin in the luminal regions of the thrombi at the expense of the base regions. These thrombi were more dependent on tissue factor-triggered thrombin generation. Microforce nanoindentation analysis revealed a significantly increased microelasticity of thrombi with luminal-oriented fibrin. At a low shear rate, fibrin fibers tended to luminally cover the thrombi, again resulting in a higher microelasticity. Studies with blood from patients with distinct hemostatic insufficiencies indicated an impairment in formation of a platelet-fibrin thrombus in the cases of dilutional coagulopathy, thrombocytopenia, Scott syndrome and hemophilia B. Taken together, our data indicate that: (i) thrombin increases the platelet thrombus volume; (ii) tissue factor drives formation of fibrin outside of the platelet thrombus; (iii) limitation of platelet adhesion redirects fibrin from bottom to top of the thrombus; (iv) a lower shear rate promotes thrombus coverage with fibrin; ( $v$ ) the fibrin distribution pattern determines thrombus microelasticity; and (vi) the thrombus-forming process is reduced in patients with diverse hemostatic defects.

\section{Introduction}

During hemostasis, blood flow and platelet activation in combined action with the coagulation system determine the formation of platelet-fibrin thrombi at the injured vessel wall. Multiple components of the damaged endothelial and subendothelial layers are known to influence the dynamics of thrombus growth and fibrin polymerization. ${ }^{1}$ Key vascular constituents triggering this process are collagen fibers and tissue factor (TF), serving as potent platelet- and coagulation-stimulating agents, respectively. ${ }^{2}$ Thrombin that is formed on the surface of activated platelets and on injured vascular cells is a central player in the hemostatic process by enhancing platelet activation, producing activated coagulation factors and converting fibrinogen into fibrin. ${ }^{3}$ Yet, the complex role of thrombin in formation of a platelet-fibrin thrombus under flow is at best incompletely studied.

Murine in vivo models of arterial thrombus formation have shown that the type and severity of vascular injury are determinative for the precise triggering of this process. Exposure of the vasculature to $\mathrm{FeCl}_{3}$, resulting in endothelial denudation, leads to collagen-dependent platelet activation with an additional role of TF, particularly upon milder $\mathrm{FeCl}_{3}$ injury. ${ }^{4-7}$ Thrombin and fibrin generation are elicited on procoagulant platelets exposing phosphatidylserine. ${ }^{8,9}$ Platelets, furthermore, control the coagulation process by secreting pro- and anticoagulant proteins, whereas there is ample evidence for a role of platelet-exposed TF. ${ }^{10,11}$ On the other hand, in most laser-induced injury 
models, thrombus formation is primarily triggered by thrombin that is generated via vascular TF. ${ }^{12,13}$ Severe laser injury, however, also leads to collagen exposure and collagen-dependent platelet activation. ${ }^{14,15}$

Together, these in vivo findings indicate that the relative availability of plateletadhesive (like collagen) and coagulation-triggering (like TF) substances determines the buildup of a thrombus and, by implication, the roles of thrombin and fibrin herein. In support of this idea, different patterns of fibrin deposition have been detected in injury models, such as fibrin structures around a thrombus, ${ }^{16}$ and thrombin/fibrin-rich areas in the thrombus core region. ${ }^{17,}{ }^{18}$ How the platelet- and coagulant-activities of the thrombogenic surface, for example, the relative abundance of collagen and TF, influence the buildup and composition of a platelet-fibrin thrombus has not been examined so far.

Viscoelasticy is considered to be an important hemostatic property of a fibrincontaining thrombus. For instance, in patients with severe factor XI deficiency, a low density fibrin network is associated with impaired hemostasis. ${ }^{19,}{ }^{20}$ However, the conditions that favor high elasticity of a platelet-fibrin thrombus or clot are largely unknown. The limited evidence available suggests that a local, high concentration of thrombin enforces the fibrin network, ${ }^{21}$ and that blood flow supports the alignment of stiff fibrin fibers. ${ }^{22,}$

${ }^{23}$ Macroscopic thromboelastic studies have not come much further than demonstrating that, in patients with dilutional coagulopathy, the overall clot strength is predictive for the risk of perioperative bleeding. ${ }^{24}$

In the present article, we investigated how the type of thrombogenic surface with defined relative amounts of collagen and TF - affects the formation and composition of platelet-fibrin thrombi that are formed under high and low shear flow conditions. We developed standardized protocols, in which citrate-anticoagulated blood was flowed over defined collagen/TF microspots, and the deposition of platelets and fibrin(ogen) were assessed simultaneously. We then determined the distribution of fibrin within and outside the thrombus by confocal microscopy, as well as the microelasticity of the formed thrombi by a novel method of nanoindentation. The data reveal a surface- and flow-dependent thrombus buildup with a different location of fibrin.

\section{Materials and Methods}

Materials and methods are available in the Supplemental data.

\section{Results}

Collagen-TF surface determines buildup of platelet-fibrin thrombus and extent of fibrin formation under flow

To study the roles of platelets and coagulation in whole blood thrombus formation under flow, we applied a previously developed microspot method of thrombus formation in parallel-plate flow chambers, ${ }^{25}$ which was adapted to operate under strictly controlled coagulant conditions. The changes included: (i) preparation of microspots with defined amounts of collagen and tissue factor (TF), (ii) co-infusion of citrate-anticoagulated 
blood with $\mathrm{Mg}^{2+} / \mathrm{Ca}^{2+}$ recalcification medium to obtain physiological concentrations of divalent cations; (iii) adequate mixing of blood with medium by pushing through a tubeslit converter in the flow chamber, and (iv) real-time confocal recording of both platelet
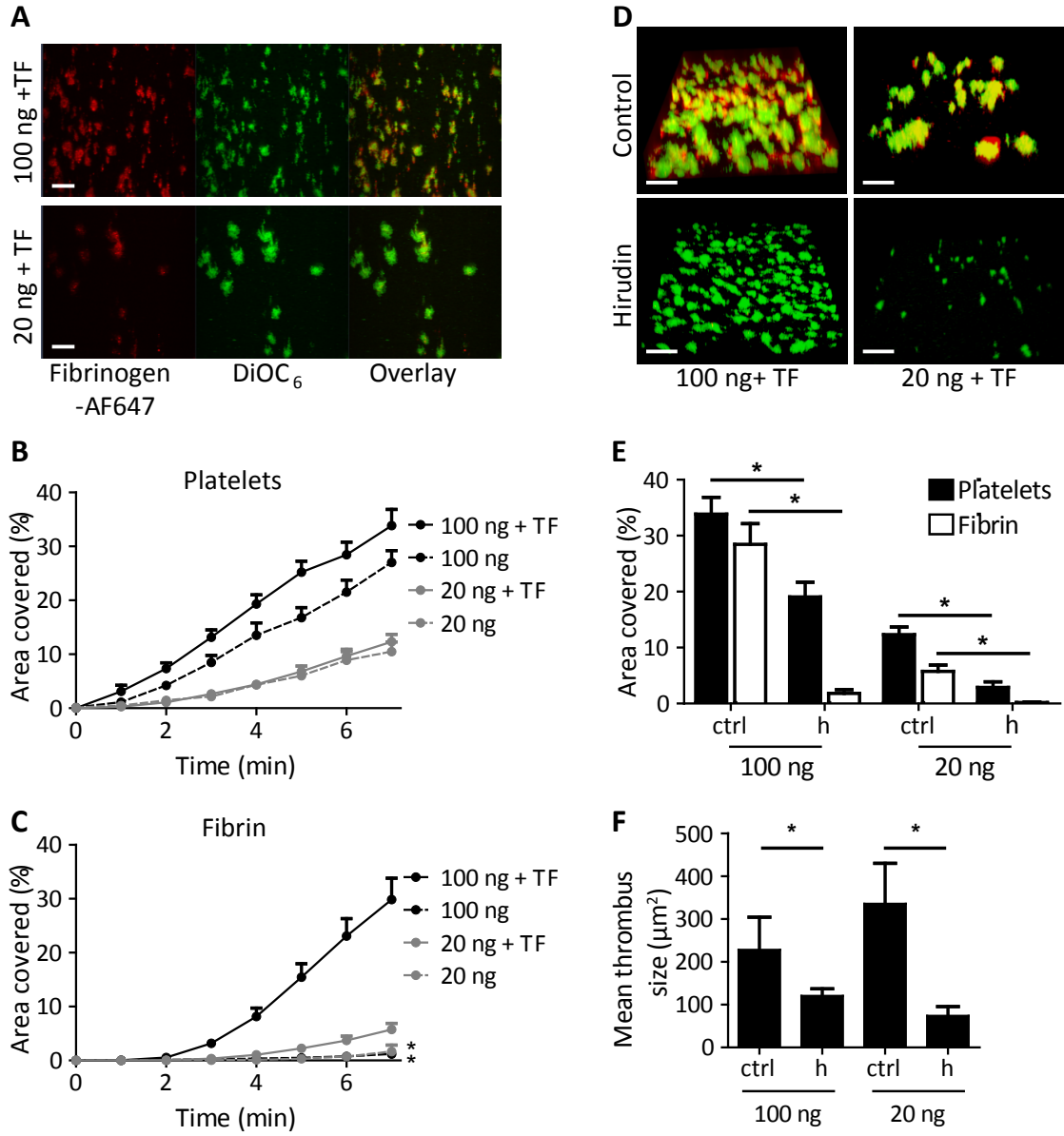

Figure 1. Collagen-tissue factor (TF) coating determines the buildup of platelet-fibrin thrombus formed under flow. Citrated whole blood was perfused during recalcification over microspots with collagen (20 or $100 \mathrm{ng})$ in the absence or presence of TF (10 pg) for 7 minutes at a wall shear rate of $1000 \mathrm{~s}^{-1}$. Blood samples were prelabeled with $\mathrm{DiOC}_{6}$ to label platelets (green) and with AF647-fibrinogen to monitor fibrin formation (red). Control samples were pretreated with hirudin (h, $3 \mu \mathrm{g} / \mathrm{mL})$, where indicated. Two-color microscopic confocal images were recorded in real time at frequency of $1 \mathrm{~Hz}$. (A) Representative fluorescence images from collagen/TF microspots after 7 minutes of flow (bars, $50 \mu \mathrm{m})$. (B, C) Time-dependent accumulation of (B) DiOC $_{6}$-labeled platelets and (C) AF647-fibrin(ogen) during blood flow. (D) Representative stacks of thrombi in 3D (z-step $1 \mu \mathrm{m})$ of platelets (green) and fibrin(ogen) (red) on collagen/TF microspots after 7 minutes of flow (bars, $50 \mu \mathrm{m}$ ). (E) Fluorescence area covered by platelets and fibrin on collagen/TF microspots after 7 minutes of flow. (F) Average thrombus size on spot as determined by morphometric image analysis. Means $\pm \operatorname{SEM}(n=6-15),{ }^{*} p<0.05$. 
deposition and fibrin generated on the microspots using 3,3'-dihexyloxacarbocyanine $\left(\mathrm{DiOC}_{6}\right)$ and Alexa Fluor (AF)647-fibrinogen as probes, which were pre-added to the blood samples (Supplemental Fig. 1).

Pilot data indicated that co-coating of $10 \mathrm{pg}$ TF per microspot resulted in optimal shortening of time to fibrin formation, as checked with bright-field image recording, such as in agreement with published findings. ${ }^{26}$ Perfusion of blood samples at a shear rate of $1000 \mathrm{~s}^{-1}$ over microspots enriched in collagen (100 ng per microspot) resulted in a rapid formation of platelet thrombi that were rich in fibrin (Fig. 1A-C). Lowering the collagen content (20 ng per microspot) significantly decreased platelet deposition after 7 minutes of perfusion (area covered, $12.3 \pm 1.4 \%$ vs. $33.8 \pm 3.0 \% ; p=0.005$ ) and delayed the onset of fibrin formation ( 3 vs. 5 minutes), as compared to the high collagen microspots (Fig. 1AC). In the absence of TF, when coagulation is triggered via the intrinsic pathway, ${ }^{27}$ platelet deposition was decreased on high collagen but unaltered on low collagen, whereas fibrin formation was greatly delayed in either case (Supplemental Table 1). Pretreatment of the

A

A Top
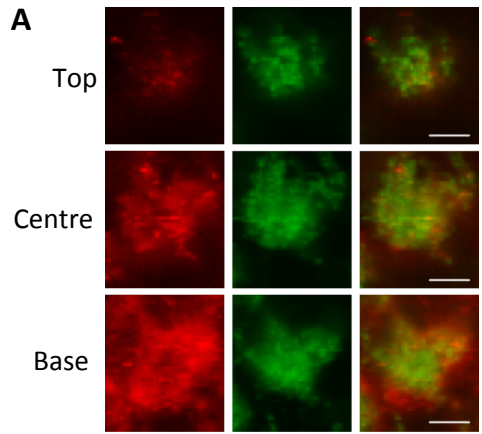

C

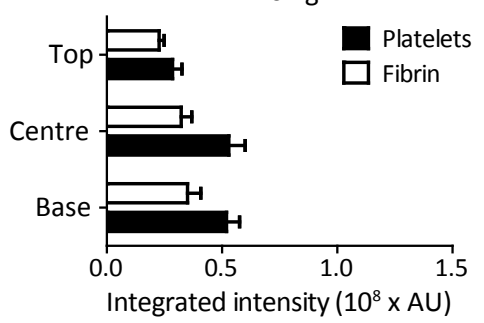

B

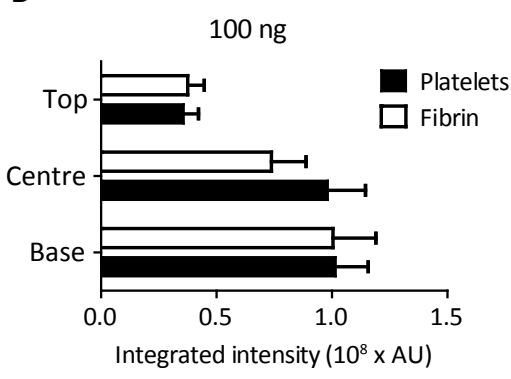

D

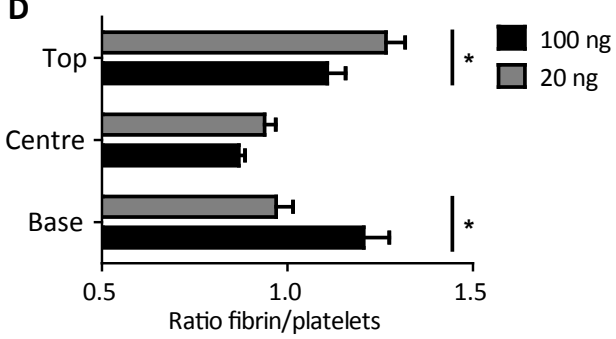

Figure 2. Collagen-tissue factor (TF) coating determines distribution of fibrin through a thrombus. Platelet-fibrin thrombi were formed by blood flow over microspots of collagen (20 or $100 \mathrm{ng}$ ) plus TF (10 pg) for 7 minutes, as described in Figure 1. Confocal images of DiOC 6 (green) and AF647-fibrin(ogen) (red) fluorescence in 3D were captured. (A) Representative fluorescence images of optical slices at base, center and top of thrombus on collagen (100 ng)/TF microspots (bars, $20 \mu \mathrm{m}$ ). (B, C) Integrated fluorescence intensity (arbitrary units, AU) of platelet (green) and fibrin (red) fluorescence from $z$ stacks at base $(<10 \mu \mathrm{m})$, center $(10-20 \mu \mathrm{m})$ and top $(>20 \mu \mathrm{m})$ of thrombi formed. (D) Ratio of platelet/fibrin fluorescence intensity at base, center and top of thrombi. Means $\pm \operatorname{SEM}(n=6-7),{ }^{*} p<0.05$. 
blood with corn trypsin inhibitor to block the intrinsic coagulation pathway resulted in a delayed formation of fibrin only for microspots not containing TF (data not shown). In the absence of collagen (only TF), neither platelets nor fibrin deposited on the surface.

Reconstruction of stacks of confocal images in 3D indicated that the platelet-fibrin thrombi formed on high collagen microspots were relatively small and dense, when compared with the more dispersed thrombi formed on low collagen microspots (Fig. $1 \mathrm{~A}, \mathrm{D})$. Persistent thrombin activity on each type of microspot was confirmed by the addition of a fluorogenic thrombin substrate, which was continuously cleaved (data not shown). Markedly, with hirudin added to the blood, platelet deposition decreased on high collagen microspots (surface area coverage from 33.8 to $19.1 \%, p=0.001$ ), whereas on low collagen hardly any platelets were left (from 12.3 to $2.9 \%, p<0.0001$ ). As expected, with hirudin present, fibrin formation was completely suppressed on either surface (Fig. 1E). Quantification also showed that hirudin significantly reduced the size of individual thrombi on the high collagen surface (from $228 \pm 76$ to $121 \pm 16 \mu \mathrm{m}^{2}, p=$ 0.007 ), a reduction that was even more pronounced on the low collagen surface (from $335 \pm 94$ to $74 \pm 21 \mu \mathrm{m}^{2}, p<0.001$ ) (Fig. 1F).

As microscopic images showed a gradual contraction of platelets in fibrincontaining thrombi, we aimed to quantify this process by addition of $3 \% \mathrm{DiOC}_{6}$-labeled washed platelets to the blood, and then monitoring the movement of adhered platelets in time. It seemed that the overall displacement rate of platelets was high on microspots with collagen/TF during the time of fibrin formation $\left(1.06 \pm 0.11 \times 10^{-3} \mu \mathrm{m} / \mathrm{s}\right)$, whereas it was non-significantly changed $\left(0.87 \pm 0.10 \times 10^{-3} \mu \mathrm{m} / \mathrm{s}\right)$ in the presence of Gly-Pro-ArgPro, inhibiting fibrin polymerization, and significantly lowered in the presence of hirudin $\left(0.64 \pm 0.05 \times 10^{-3} \mu \mathrm{m} / \mathrm{s}, p=0.0064\right)$. Hence, under the present flow conditions, platelet contraction did occur in the absence of thrombin or fibrin but was enhanced by the formation of either. This resolves a dispute in the literature that platelet-fibrin retraction is considered a thrombin-dependent event ${ }^{28}$ but can still occur under conditions where thrombin is blocked. ${ }^{29}$

By lowering the wall shear rate from 1000 to $150 \mathrm{~s}^{-1}$, platelet deposition on high or low collagen was reduced (Supplemental Table 1), as expected due to a diminished role of von Willebrand factor. ${ }^{25}$ On the other hand, total fibrin formation did not reduce at lower shear rate (Supplemental Fig. 2). Co-localization analysis of fluorescence from platelets and fibrin indicated major extension of the fibrin fibers outside of platelet aggregates especially at low shear conditions $\left(150 \mathrm{~s}^{-1}\right)$, and to a lesser extent at the higher shear rate (Supplemental Fig. 2). Jointly, these data indicate that limitation of the plateletadhesive collagen surface resulted in an overall reduced platelet deposition, which was partly compensated by a larger thrombus size because of the activity of TF-triggered thrombin generation. Lowering of the shear rate also reduced platelet accumulation but in a relative way promoted fibrin formation.

\section{Collagen-TF surface and shear rate define distribution of fibrin in a thrombus}

To assess the amount and localization of thrombus-associated fibrin on different microspots, $z$ stacks of confocal images of the fluorescent-labeled platelets and fibrin were captured at the end of high shear flow experiments (Fig. 2A). On microspots with 
high collagen, the majority of platelet and fibrin fluorescence was located near the base $(0-10 \mu \mathrm{m})$ and center regions $(10-20 \mu \mathrm{m})$ of the thrombi. On microspots with low collagen, overall thrombus volume was reduced from $1.10 \pm 0.20$ to $0.46 \pm 0.10 \times 10^{6} \mu \mathrm{m}^{3}$ $(p<0.001$; Supplemental Table 1). In this case, fluorescence from platelets and fibrin was more evenly distributed among the base, center and top regions of the thrombus (Fig. 2B, $C)$. Markedly, the top regions of thrombi formed on low collagen showed a significantly higher ratio of fibrin to platelet fluorescence $(p=0.022)$, compared with those on high collagen (Fig. 2D). In contrast, the base regions of thrombi formed on low collagen had a lower fibrin/platelet ratio $(p=0.013)$, compared to the high collagen microspots.

The images were also analyzed for co-localization of platelets and fibrin, in order to assess the appearance of fibrin outside the aggregated platelets. On high collagen microspots, more fibrin fibers extended outside the platelet area at the base of the thrombi ( $28.8 \pm 1.9 \%$ of fibrin-positive pixels) than at the center and top regions of the thrombus ( $8.9 \pm 2.1 \%$ and $5.3 \pm 1.8 \%$ fibrin-positive pixels, respectively; $p<0.001$ ). Fibrin outgrowth was further restricted on low collagen microspots, where only $9.7 \pm$ $2.8 \%, 2.3 \pm 0.6 \%$ and $5.2 \pm 1.1 \%$ of the fibrin fluorescence were detected outside the platelet aggregates, at the base, center or top regions of thrombi, respectively.

Interestingly, when compared with a high shear rate, at a low shear rate of $150 \mathrm{~s}^{-1}$, overall thrombus volume reduced from $1.10 \pm 0.20$ to $0.78 \pm 0.02 \times 10^{6} \mu \mathrm{m}^{3}(p=0.032)$ on high collagen and from $0.46 \pm 0.10$ to $0.29 \pm 0.04 \times 10^{6} \mu \mathrm{m}^{3}(p=0.026)$ on low collagen. At low shear rate, the majority of the fibrin was located outside of the platelet regions, which were low in height, such as apparent from analysis of z stacks of confocal images (Supplemental Table 1).
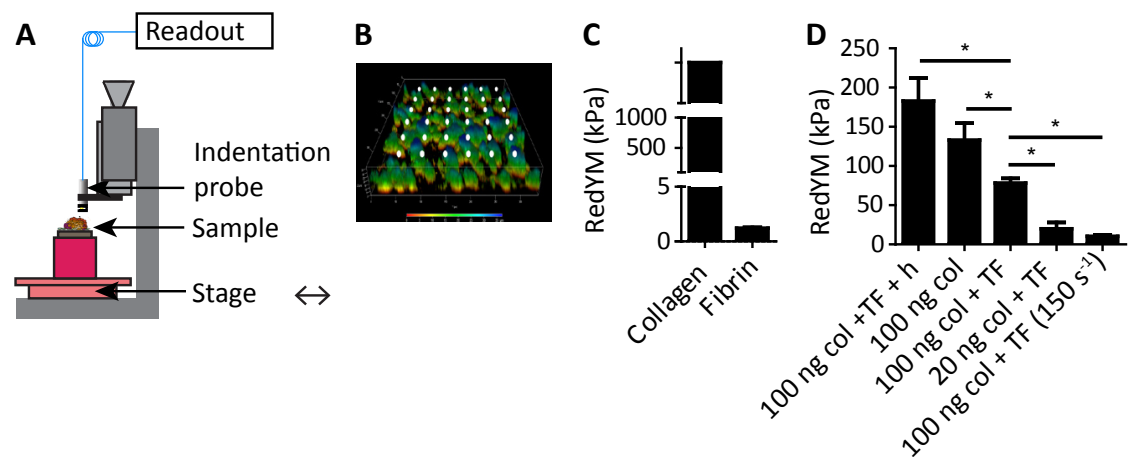

Figure 3. Collagen-tissue factor (TF) coating determines microelasticity of platelet-fibrin thrombus formed under flow. Platelet-fibrin thrombi were formed on microspots with collagen $\pm T F$, as described in Figure 1. Flow experiments were carried out at shear rate of $1000 \mathrm{~s}^{-1}$, except where indicated otherwise $\left(150 \mathrm{~s}^{-1}\right)$. After careful rinse with Hepes buffer $\mathrm{pH} 7.5$, coverslips with thrombi were subjected to nanoindentation (6 $x 6$ indentations/microspot). Control coverslips were used for scanning electron microscopy. (A) Schematic presentation of nanoindenter, featuring the indentation probe, sample and automated $x-y$ stage. (B) Schematic grid pattern of preset $6 \times 6$ indentations per microspot with height gradient. (C) Reduced Young's modulus (RedYM, $\mathrm{kPa}$ ), a parameter inversely related to the microelasticity, in control measurements with a collagen-coated surface or with fibrinated plasma $(n=16)$. (D) RedYM assessed for thrombi formed on indicated collagen/TF surfaces $(n=36-72)$. Means \pm SEM, $* p<0.05$, h indicates hirudin. 


\section{Collagen-TF surface and shear rate influence microelasticity of a thrombus}

A novel method of ferrule-top nanoindentation, related to atomic force microscopy, was used to determine how the quantity and localization of fibrin fibers affected the mechanical and microelastic properties of the thrombi formed on microspots. This method is schematized in Fig. $3 \mathrm{~A}$, and described in more detail in the Supplement data. By gradually indenting and retracting a nanoindentation tip at multiple spots per coverslip, loading and unloading curves could be obtained, wherein slopes of the unloading curves informed on the reduced Young's modulus (RedYM), as an inverse measure for the microelasticity of the sample (Supplemental Fig. 3). By applying multiple nanoindentations per microspot surface in a grid-like pattern, high-resolution information on the microelasticity of the thrombi on the surface could be obtained (Fig. 3B). Uncoated (not shown) or collagen-coated coverslips without thrombi gave a high RedYM of $>1000$ kPa (Fig. 3C). In contrast, coverslips covered with coagulated, fibrin-containing plasma produced a low RedYM of $1.22 \pm 0.1 \mathrm{kPa}$, thus pointing to high microelasticity of the fibrin network. Nanoindentation measurements of thrombi formed on high collagen (78.2 \pm $5.9 \mathrm{kPa})$ and low collagen $(20.0 \pm 8.1 \mathrm{kPa})$ microspots resulted in different RedYM values $(p=0.019)$, indicating that the latter had a higher microelasticity (Fig. 3D). Similarly, for thrombi formed on high collagen, lowering of the shear rate from $1000 \mathrm{~s}^{-1}$ to $150 \mathrm{~s}^{-1}$ resulted in a lower RedYM $(p<0.0001)$ and hence a higher microelasticity (Fig. 3D). This suggested that intrathrombus fibrin increased the microelasticity of the sample. This was confirmed by nanoindentation analysis of fibrin-poor thrombi, formed on collagen without TF, giving high RedYM values of $133.1 \pm 21.6 \mathrm{kPa}$. In the presence of hirudin to block thrombin activity, and hence fibrin formation, the RedYM even further increased to $182.9 \pm 29.3 \mathrm{kPa}$ (Fig. 3D). Together, this indicates that the thrombi with fibrin in the top or with outside coverage of fibrin, being formed on low density collagen microspots or at low shear rate, display a higher microelasticity, when compared with thrombi in which fibrin is accumulated at the base.

\section{Formation of platelet-fibrin thrombus under conditions of impaired hemostasis}

Using several approaches, we further assessed the roles of platelets and other blood components in the formation of fibrin-containing thrombi. Considering the diminished clotting activity of patients with perioperative dilutional coagulopathy, ${ }^{30,}{ }^{31}$ we first determined the consequences of blood dilution in vitro. Blood samples were flowed at a shear rate of $1000 \mathrm{~s}^{-1}$ over high collagen/TF microspots. In the absence of dilution, this resulted in a high platelet deposition and fibrin formation (see above). However, dilution of the blood to 80,60 and $40 \%$ resulted in a gradual decrease in platelet deposition after 7 minutes of perfusion, amounting to $14 \pm 2 \%(p=0.046), 10 \pm 3 \%(p=0.004)$ and $8 \pm 3 \%$ $(p<0.001)$, respectively, in comparison with the undiluted sample. In contrast, amounts of fibrin only reduced at dilution to 60 and $40 \%$ blood, which reduction was accompanied by a prolonged time to onset of fibrin formation (Fig. 4A, B). Comparable results were obtained under conditions of a low, venous shear rate of $150 \mathrm{~s}^{-1}$ (Supplemental Fig. 4). Reconstitution of the $40 \%$ diluted blood with either washed platelets or red blood cells 

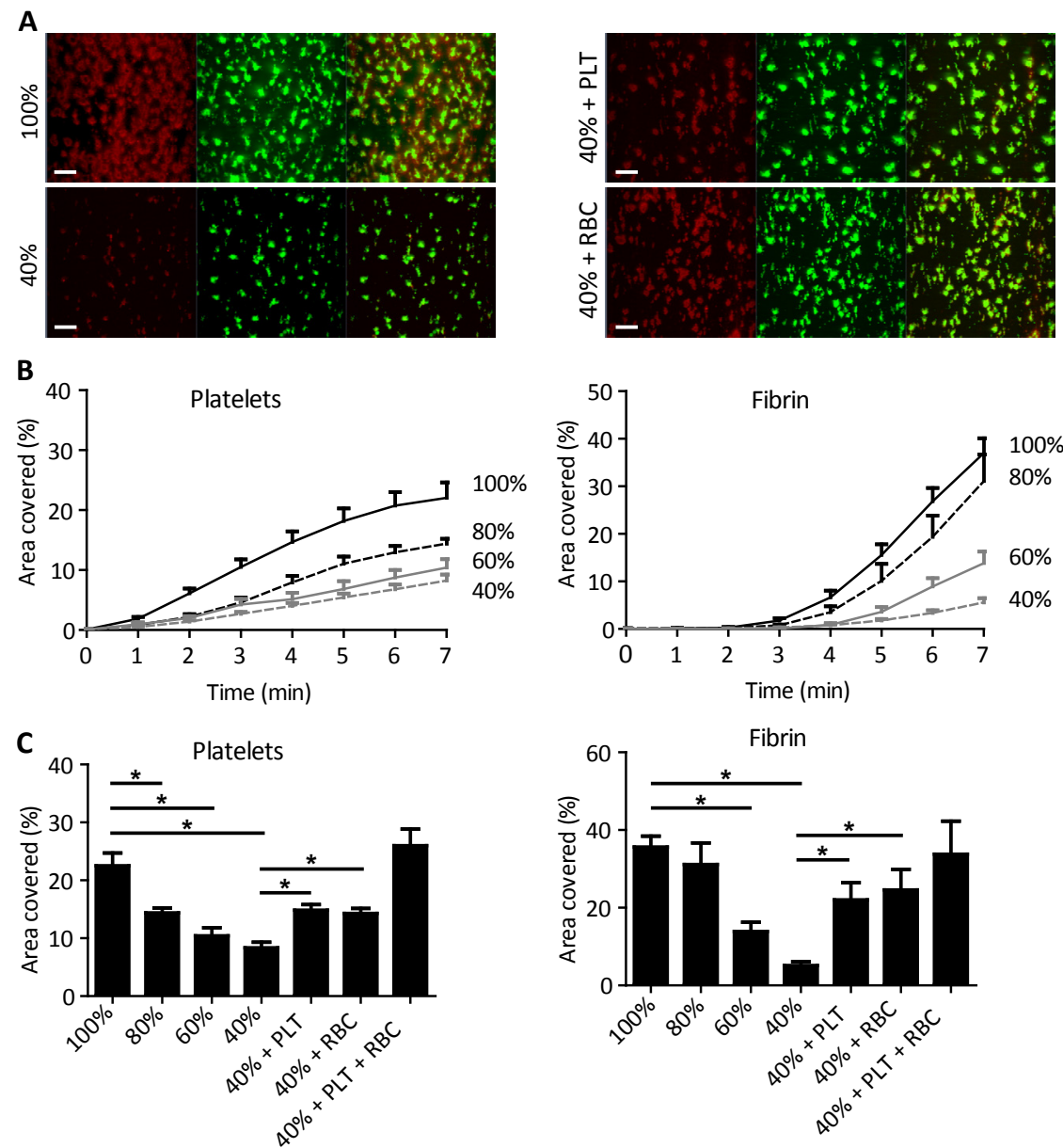

Figure 4. Restoration of platelet-fibrin thrombus formation by reconstitution of diluted blood. Plateletfibrin thrombi were formed on microspots with collagen (100 ng) and tissue factor (TF; $10 \mathrm{pg}$ ), as described in Figure 1. Blood samples were diluted to indicated percentages with saline (keeping fixed concentrations of citrate, $\mathrm{MgCl}_{2}$ and $\mathrm{CaCl}_{2}$ ). Specific samples were reconstituted with autologous washed platelets (PLT) or red blood cells (RBC), added to the original counts. (A) Representative fluorescence images of DiOC $_{6}$-labeled platelets (green) and AF647-fibrin(ogen) (red) after 7 minutes of flow using $100 \%$ or $40 \%$ blood with(out) reconstitution (bars, $50 \mu \mathrm{m}$ ). (B) Time-dependent accumulation of fluorescence from platelets (green) and fibrin (red) during perfusion. (C) Fluorescence area covered after 7 minutes of flow by platelets and fibrin. Means $\pm \operatorname{SEM}(n=5-12),{ }^{*} p<0.05$.

caused partial improvement of both platelet deposition and fibrin formation (Fig. 4A, C). Interestingly, complete recovery of the thrombus-forming process was obtained by addition of platelets in combination with red blood cells (Fig. 4C). These data pointed to 
A

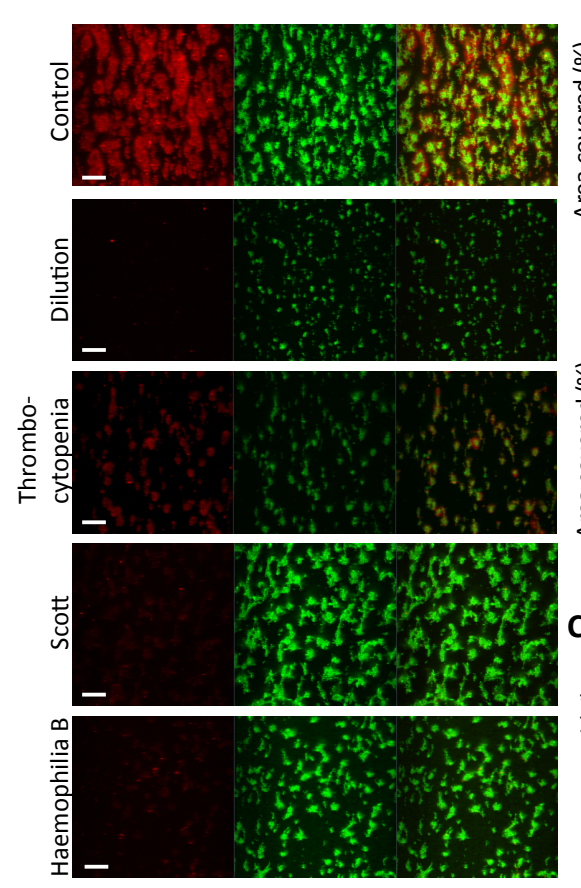

B
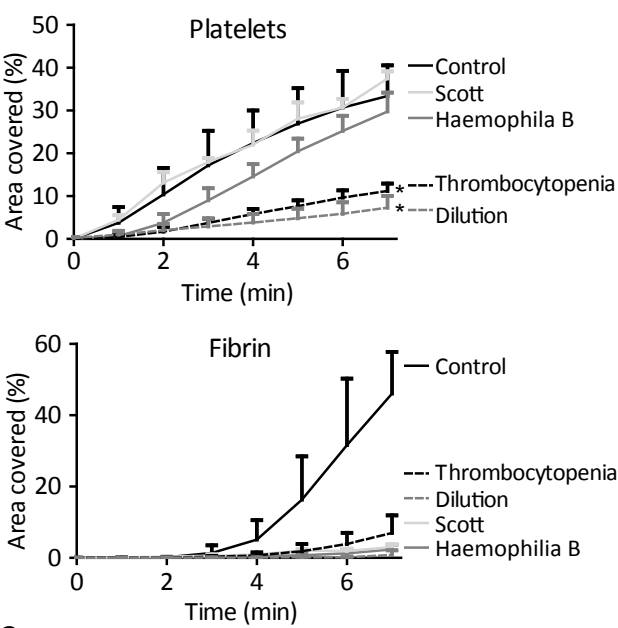

C

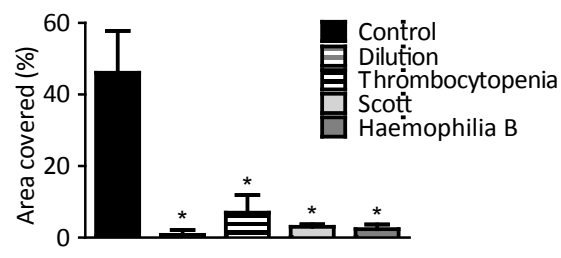

Figure 5. Impaired platelet-fibrin thrombus formation using blood from patients with impaired hemostasis. Blood was obtained from 7 healthy control subjects, 3 patients with dilutional coagulopathy because of massive fluid infusion during cardiothoracic surgery, 2 patients with acquired thrombocytopenia, 1 Scott syndrome, or 2 patients with hemophilia B. At least 3 blood samples were analyzed per condition. Plateletfibrin thrombi were formed in a standard way by blood flow at $1000 \mathrm{~s}^{-1}$ over microspots with collagen (100 $\mathrm{ng}$ ) and TF (10 pg), as described in Figure 1. (A) Representative images of DiOC 6 -labeled platelets (green) and AF647-fibrin(ogen) (red) after 7 minutes (bars, $50 \mu \mathrm{m}$ ). (B) Time-dependent accumulation of platelet and fibrin fluorescence during blood perfusion. (C) Fluorescence area covered by fibrin after 7 minutes of flow. Means \pm SD $(n=6-9)$, *, significantly different from reference range of control blood samples.

a limiting role of platelet adhesion (enforced by marginalization with erythrocytes) not only for platelet aggregation but also for fibrin formation under conditions of high shear flow. This was confirmed with scanning electron microscopy (Supplemental Fig. 5).

As a second approach, blood was used from various patients with hemostatic insufficiencies and a bleeding risk. Blood samples were obtained from 3 patients with dilutional coagulopathy because of massive fluid infusion during surgery. In this case, only few platelet aggregates of small size were formed and no fibrin was generated during the measured time of 7 minutes (Fig. 5A). With blood samples from 2 thrombocytopenic patients, platelet deposition was comparably low, although measurable amounts of fibrin were formed (Fig. 5A, C), such in accordance with normal coagulation activity in this condition. A requirement for platelet procoagulant activity (phosphatidylserine exposure) was investigated with blood samples from a Scott patient, whose platelets lack this property. ${ }^{32}$ Whereas platelet deposition and aggregate size were not affected, 

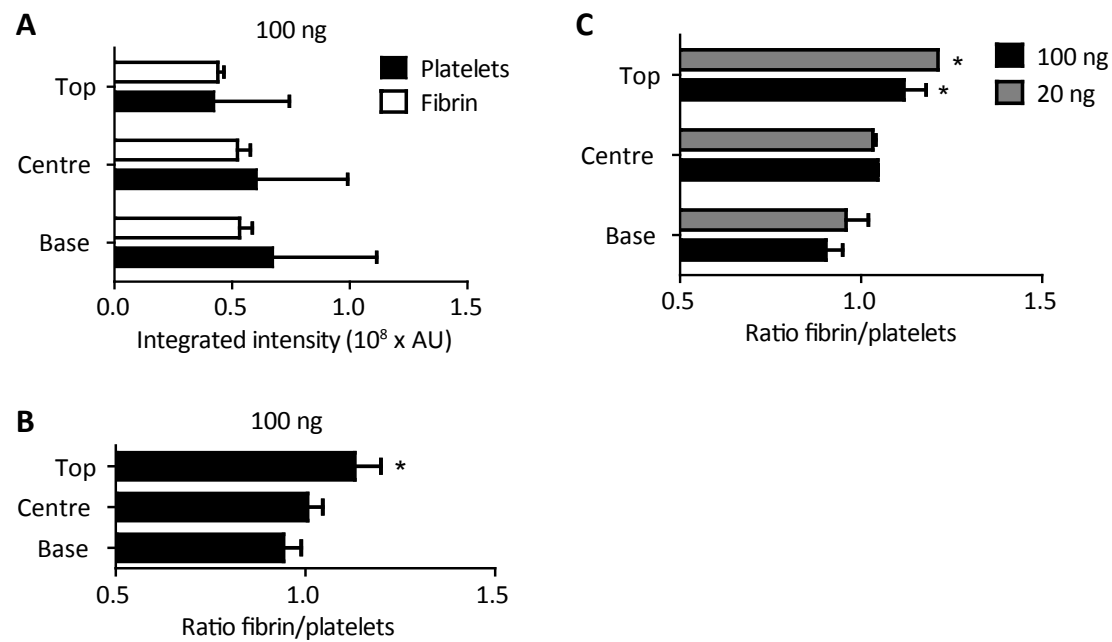

Figure 6. Altered fibrin distribution within thrombus formed by thrombocytopenic blood. Platelet-fibrin thrombi were formed by blood flow over microspots with collagen (20 or $100 \mathrm{ng}$ ) and tissue factor (TF; 10 $\mathrm{pg}$ ), as described in Figure 1. Confocal images of $\mathrm{DiOC}_{6}$ (green) and AF647-fibrin(ogen) staining (red) in 3D were captured. (A) Integrated fluorescence intensity (arbitrary units, AU) of platelet (green) and fibrin (red) fluorescence from $z$ stacks at base $(>10 \mu \mathrm{m})$, center $(10-20 \mu \mathrm{m})$ and top $(>20 \mu \mathrm{m})$ regions of thrombi after 7 minutes of blood perfusion on high collagen/TF. (B, C) Ratio of platelet/fibrin staining at base, center and top of thrombi after 7 minutes on high collagen (B) or after 12 minutes (C) of blood perfusion on low and high collagen. Means $\pm \mathrm{SD}(n=3),{ }^{*} p<0.05$ vs. base.

fibrin formation was essentially absent in the Scott blood samples. Similarly, impaired coagulation, as in blood from 2 patients with hemophilia B ( $5 \%$ factor IX), led to normal platelet aggregation under flow, but an impaired fibrin formation (Fig. 5A, C).

Because the flow of thrombocytopenic blood over high collagen microspots still gave limited fibrin formation, the intrathrombus localization of fibrin could be analyzed in more detail. Analysis of $z$ stacks of confocal images indicated that fibrin and platelets were similarly distributed throughout the thrombi (Fig. 6A). Yet, the ratio of fibrin to platelets was higher in the top regions of thrombi (Fig. 6B), with only limited fibrin extending from the platelet aggregates at base areas ( $12 \pm 8 \%$ of fibrin-positive pixels). This fibrin distribution pattern, concentrated in platelet regions, persisted even after prolongation of the perfusion time to 12 minutes (Fig. 6C). After 12 minutes, at high and low collagen microspots, fibrin deposition outside the platelet regions at the thrombus base gradually increased to $34 \pm 14 \%$ and $6 \pm 1 \%$, respectively. Jointly, these results pointed to platelet control of fibrin formation and distribution in thrombi formed on collagen/TF surfaces, relying on phosphatidylserine exposure.

\section{Discussion}

This study shows that, under conditions of high shear blood flow and coagulation, platelet deposition and platelet-dependent fibrin formation are decreased when collagen as a platelet-activating substrate becomes limited. Although fewer thrombi are formed 
on microspots with low collagen, we see a partly compensating effect in that thrombus size is increased and the contribution of thrombin to thrombus growth is relatively more important. Nanoindentation measuring viscoelastic properties of the sample indicates that the thrombi formed on low collagen possess enhanced microelasticity because of fibrin accumulation in the luminal region of thrombi. Interestingly, redistribution of fibrin from the base to the luminal region of thrombi is also observed under conditions of thrombocytopenia. Hence, a limitation of either the platelet-activating surface or the platelet number leads to reductions in platelet deposition and fibrin accumulation, which effects are accompanied by an altered fibrin distribution throughout the thrombus. Blood flow at low shear rate similarly enhances microelasticity by reducing platelet deposition and increasing the formation of a fibrin coat surrounding the thrombi.

\section{Model of thrombus buildup, determining fibrin distribution and microelasticity}

Platelet adhesion to collagen is known to result in a sustained rise in cytosolic calcium and phosphatidylserine exposure. This procoagulant surface greatly promotes the assembly of coagulation factors culminating in the formation of factor $\mathrm{Xa}$ and thrombin. ${ }^{2}$ Earlier, in flowing mouse blood, we have established that in the presence of collagen and TF this platelet-dependent factor Xa formation is essential for the propagation of fibrin formation and for boosting platelet phosphatidylserine exposure. ${ }^{3}$ The present results allow to extend this coagulation-platelet activation cross talk to the human system, in that we find that human deficiency in factor IX (hemophilia B patient) or deficiency in platelet phosphatidylserine exposure (Scott syndrome patient) results in almost complete abolition of fibrin formation under flow conditions.

Although in the past years progress has been made in determining the mechanical properties of fibrin fibers formed under static conditions, little is known about the elasticity of platelet-fibrin thrombi formed under flow. Recently, a new method of nanoindentation has been used to assess the microelasticity of clotted murine platelet-rich plasma. ${ }^{33}$ In this study, we have used this technique to measure the physical characteristics of human thrombi formed at high and low shear rates and find that thrombi with a high fibrin content in the thrombus top region are highest in microelasticity. Jointly, our results indicate that not so much the fibrin content, but rather the fibrin distribution through a thrombus determines its elastic properties. We hypothesize that the high microelasticity of thrombi with luminal-oriented fibrin ameliorates the hemostatic process. In agreement with this, thromboelastometry studies have indicated that a high clot elasticity associates with less bleeding in patients with hemostatic insufficiencies. ${ }^{20,34}$ If platelet adhesion is limited (low collagen), we see a relatively high fibrin content in the top region of an apparently 'loose' thrombus. On the other hand, at high platelet adhesion (high collagen), fibrin appears to be primarily formed at the base region of thrombi. Summarizing these data as in Figure 7, we conclude that: (i) the presence of thrombin increases platelet-thrombus volume independent of the shear rate; (ii) the presence of TF drives fibrin formation outside of the thrombus; (iii) limitation of platelet adhesion redirects fibrin from the bottom to the 


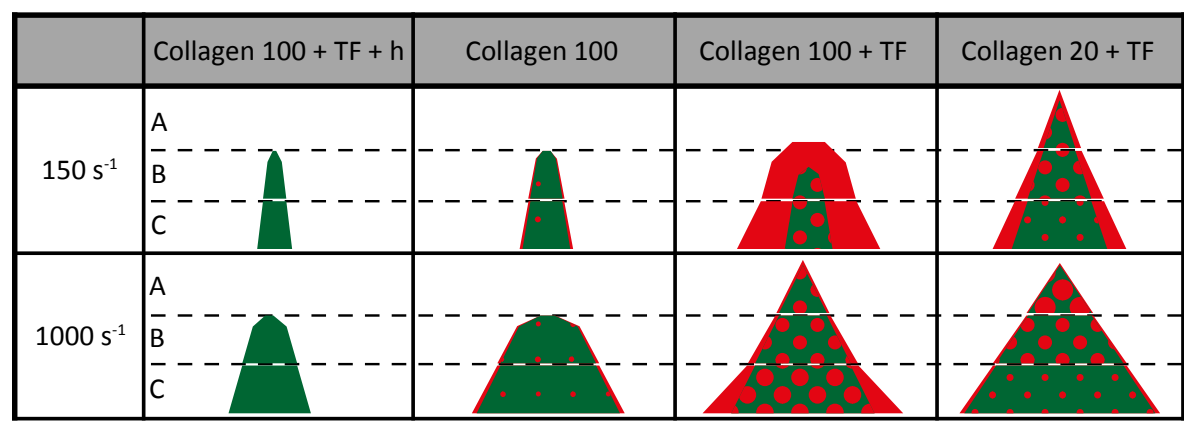

Figure 7. Schematic representation of thrombus buildup on collagen/TF surfaces. Platelet-fibrin thrombi were formed by blood perfusion over microspots with indicated amounts of collagen and TF at indicated shear rates, as described in Figure 1. In specific blood samples, thrombin was inhibited with hirudin $(+h)$. Multiple parameters of thrombus formation were assessed; for full data see Supplemental Table 1. Cartoons illustrate key characteristics of thrombi in terms of: width (scale 1-5), height (scale 1-3), fibrin inside platelet thrombus (sphere size: 1-4), and fibrin outside platelet thrombus (size of shell). Presentation is for three thrombus zones: A, $<10 \mu \mathrm{m}$ from microspot surface; B, 10-20 $\mu \mathrm{m} ;$ C, $>20 \mu \mathrm{m}$.

top of the thrombus; (iv) lowering of the shear rate results in a more fibrin rich thrombus; and $(v)$ the thrombus microelasticity is determined by the distribution pattern of fibrin.

\section{Comparison to in vivo models}

These findings provide an extension of the hemostatic thrombus model, based on in vivo observations, presented by the groups of Brass and Diamond, in which thrombin activity is confined to the dense thrombus core. ${ }^{18,35,36}$ Previous in vivo studies have shown that, upon laser-induced injury of arterioles, where exposed TF drives the thrombotic process, fibrin concentrates near the vessel wall and in the vascular-oriented part of the thrombus. ${ }^{6,12,14}$ Our findings suggest that this vascular-oriented fibrin distribution pattern points to a relatively high abundance of platelet-adhesive substrates. These substrates can be collagens, as well as other vascular components, such as laminins and von Willebrand factor. In other words, it seems that the 'strength' of the plateletadhesive surface controls the platelet packing density during thrombus buildup, and thereby the thrombin retention and fibrin distribution pattern. Confirmative evidence for this hypothesis comes from in vivo studies using PAR4-deficient mice, in which fibrin was found to redistribute through the whole thrombus as a consequence of diminished platelet activation. ${ }^{16}$

A different pattern of fibrin distribution occurs under low shear flow conditions, where relatively more (microelastic) fibrin is formed, appearing as a coat that covers the thrombi. This might be explained by a reduction in the flow-dependent removal of thrombin and fibrin monomers. ${ }^{37}$ In accordance with our results, it has been shown that the 
thrombi formed in mesenteric venules after laser injury are surrounded by a fibrin cap. ${ }^{38}$

\section{Effects of hemostatic insufficiencies}

Under conditions of (perioperative) dilution, we find that platelet deposition is more sensitive to blood dilution than fibrin formation, which is impaired already at $60 \%$ blood. This contrasts to static measurements of macroscopic clot strength by thromboelastometry, which seemed to be less sensitive to dilution effects. ${ }^{30}$ Singlepass flow perfusion likely makes the difference here because under flow the deposited platelets provide a rate-limiting surface for the formation of thrombin and subsequent fibrin, whereas the same flow removes procoagulant factors and hence restricts fibrin formation. ${ }^{37}$ Remarkably, in reconstitution experiments, the addition of both platelets and red blood cells was required for complete reversal of dilution effects. By implication, this indicates that under conditions of flow and sufficient margination of platelets by red blood cells, a coagulant activity of $>60 \%$ is required for unrestricted generation of fibrin. These findings are supported by hydrodynamic simulations indicating that platelet interactions with the vessel wall are promoted by an increasing hematocrit. ${ }^{39}$

A limitation of the present study is the absence of vascular cells such as endothelial cells, which can also provide a procoagulant surface for the formation of thrombin. ${ }^{40}$ On the other hand, current overviews indicate that collagen (TF)-based in vitro flow studies compare well with in vivo arterial thrombosis models, when evaluating the functional effects of genetic knockout in mice. ${ }^{41,42}$ Hence, we consider the present test of formation of platelet-fibrin thrombi under flow as a valid method to determine the hemostatic capacity of a blood sample. For fibrin localization we used fluorescently labeled fibrinogen and therefor the distinction between fibrinogen and fibrin cannot be made unambiguously. We minimized the contribution of fibrinogen to the fibrin signal by threshold settings and the presence of fluorescent fibrin fibers was always confirmed from bright-field images. The advantage is that this method prevents underestimation of the fibrin signal as a consequence of poor permeation in the thrombus when using a fibrin antibody.

In summary, we conclude that under conditions of flow and coagulation the amount and localization of fibrin are regulated by the relative abundance of triggers for platelet adhesion/activation (e.g. collagen) and coagulation (TF), and that the fibrin distribution is determinative for the clot microelasticity. Thrombin herein influences thrombus growth as well as fibrin formation, depending on its local concentration.

\section{Sources of funding}

Support for this work was obtained from the Dutch Landsteiner Foundation for Blood Transfusion Research (1006) and ZonMW, Animal-free Research Techniques (114021004). PvdM is supported by the Cardiovascular Center Maastricht.

\section{Disclosures}

NR, EJB and KL are employees of Optics11, Amsterdam, The Netherlands. Other authors have nothing to disclose. 


\section{Significance}

Activation of blood platelets as well as the coagulation system is required for a normal hemostatic response. While it is known that platelet- and fibrin-dependent thrombus formation is regulated by blood flow, the blood composition and vascular triggers such as collagen and tissue factor, the interactions between these bloodborne and vascular components are not well understood. Our data imply that the relative abundance of vascular triggers of platelet adhesion/activation (collagen) at one side and of coagulation (tissue factor) at the other side is determining for both thrombus growth and thrombus composition, in particular regarding the distribution of fibrin. Furthermore, we show that the fibrin distribution is determinative for the thrombus microelasticity as assessed by nanoindentation. 


\section{References}

1. Wolberg AS, Aleman MM, Leiderman K, Machlus KR. Procoagulant activity in hemostasis and thrombosis: Virchow's triad revisited. Anesth Analg. 2012;114:275-285.

2. Versteeg HH, Heemskerk JW, Levi M, Reitsma PH. New fundamentals in hemostasis. Physiol Rev. 2013;93:327-358.

3. Swieringa F, Kuijpers MJ, Lamers MM, Van der Meijden PE, Heemskerk JW. Rate-limiting roles of the tenase complex of factors VIII and IX in platelet procoagulant activity and formation of platelet-fibrin thrombi under flow. Haematologica. 2015;100:748-756.

4. Nieswandt B, Brakebusch C, Bergmeier W, Schulte V, Bouvard D, Mokhtari-Nejad R, Lindhout T, Heemskerk JW, Zirngibl H, Fassler R. Glycoprotein VI but not $\alpha_{2} \beta_{1}$ integrin is essential for platelet interaction with collagen. Embo J. 2001;20:2120-2130.

5. Massberg S, Gawaz M, Gruner S, Schulte V, Konrad I, Zohlnhofer D, Heinzmann U, Nieswandt B. A crucial role of glycoprotein VI for platelet recruitment to the injured arterial wall in vivo. J Exp Med. 2003;197:41-49. 6. Dubois C, Panicot-Dubois L, Merrill-Skoloff G, Furie B, Furie BC. Glycoprotein VI-dependent and -independent pathways of thrombus formation in vivo. Blood. 2006;107:3902-3906.

7. Kuijpers MJ, Munnix IC, Cosemans JM, Vlijmen BV, Reutelingsperger CP, Egbrink MO, Heemskerk JW. Key role of platelet procoagulant activity in tissue factor-and collagen-dependent thrombus formation in arterioles and venules in vivo differential sensitivity to thrombin inhibition. Microcirculation. 2008;15:269282.

8. Munnix IC, Kuijpers MJ, Auger J, Thomassen CM, Panizzi P, Van Zandvoort MA, Rosing J, Bock PE, Watson SP, Heemskerk JW. Segregation of platelet aggregatory and procoagulant microdomains in thrombus formation: regulation by transient integrin activation. Arterioscler Thromb Vasc Biol. 2007;27:2484-2490.

9. De Witt SM, Verdoold R, Cosemans JM, Heemskerk JW. Insights into platelet-based control of coagulation. Thromb Res. 2014;133 Suppl 2:S139-148.

10. Panes O, Matus V, Saez CG, Quiroga T, Pereira J, Mezzano D. Human platelets synthesize and express functional tissue factor. Blood. 2007;109:5242-5250.

11. Schwertz H, Tolley ND, Foulks JM, Denis MM, Risenmay BW, Buerke M, Tilley RE, Rondina MT, Harris EM, Kraiss LW, Mackman N, Zimmerman GA, Weyrich AS. Signal-dependent splicing of tissue factor pre-mRNA modulates the thrombogenicity of human platelets. J Exp Med. 2006;203:2433-2440.

12. Falati S, Gross P, Merrill-Skoloff G, Furie BC, Furie B. Real-time in vivo imaging of platelets, tissue factor and fibrin during arterial thrombus formation in the mouse. Nat Med. 2002;8:1175-1181.

13. Furie B, Furie BC. Mechanisms of thrombus formation. N Engl J Med. 2008;359:938-949.

14. Hechler B, Nonne C, Eckly A, Magnenat S, Rinckel JY, Denis CV, Freund M, Cazenave JP, Lanza F, Gachet C. Arterial thrombosis: relevance of a model with two levels of severity assessed by histologic, ultrastructural and functional characterization. J Thromb Haemost. 2010;8:173-184.

15. Bellido-Martin L, Chen V, Jasuja R, Furie B, Furie BC. Imaging fibrin formation and platelet and endothelial cell activation in vivo. Thromb Haemost. 2011;105:776-782.

16. Vandendries ER, Hamilton JR, Coughlin SR, Furie B, Furie BC. Par4 is required for platelet thrombus propagation but not fibrin generation in a mouse model of thrombosis. Proc Natl Acad Sci U S A. 2007;104:288292.

17. Stalker TJ, Traxler EA, Wu J, Wannemacher KM, Cermignano SL, Voronov R, Diamond SL, Brass LF. Hierarchical organization in the hemostatic response and its relationship to the platelet-signaling network. Blood. 2013;121:1875-1885.

18. Stalker TJ, Welsh JD, Tomaiuolo M, Wu J, Colace TV, Diamond SL, Brass LF. A systems approach to hemostasis: 3 . Thrombus consolidation regulates intrathrombus solute transport and local thrombin activity. Blood. 2014;124:1824-1831.

19. Wolberg AS. Thrombin generation and fibrin clot structure. Blood Rev. 2007;21:131-142.

20. Zucker M, Seligsohn U, Salomon O, Wolberg AS. Abnormal plasma clot structure and stability distinguish bleeding risk in patients with severe factor XI deficiency. J Thromb Haemost. 2014;12:1121-1130.

21. Neeves KB, Illing DA, Diamond SL. Thrombin flux and wall shear rate regulate fibrin fiber deposition state during polymerization under flow. Biophys J. 2010;98:1344-1352.

22. Collet JP, Shuman H, Ledger RE, Lee S, Weisel JW. The elasticity of an individual fibrin fiber in a clot. Proc Natl Acad Sci U S A. 2005;102:9133-9137. 
23. Gersh KC, Edmondson KE, Weisel JW. Flow rate and fibrin fiber alignment. J Thromb Haemost. 2010;8:2826-2828.

24. Schols SE, Lancé MD, Feijge MA, Damoiseaux J, Marcus MA, Hamulyák K, Ten Cate H, Heemskerk JW, Van Pampus EC. Impaired thrombin generation and fibrin clot formation in patients with dilutional coagulopathy during major surgery. Thromb Haemost. 2010;103:318-328.

25. De Witt SM, Swieringa F, Cavill R, Lamers MM, Van Kruchten R, Mastenbroek T, Baaten C, Coort S, Pugh N, Schulz A, Scharrer I, Jurk K, Zieger B, Clemetson KJ, Farndale RW, Heemskerk JW, Cosemans JM. Identification of platelet function defects by multi-parameter assessment of thrombus formation. Nat Commun. 2014;5:4257.

26. Okorie UM, Denney WS, Chatterjee MS, Neeves KB, Diamond SL. Determination of surface tissue factor thresholds that trigger coagulation at venous and arterial shear rates: amplification of $100 \mathrm{fM}$ circulating tissue factor requires flow. Blood. 2008;111:3507-3513.

27. Van der Meijden PE, Munnix IC, Auger JM, Govers-Riemslag JW, Cosemans JM, Kuijpers MJ, Spronk HM, Watson SP, Renné T, Heemskerk JW. Dual role of collagen in factor XII-dependent thrombus formation. Blood. 2009;114:881-890.

28. Shattil SJ, Kim C, Ginsberg MH. The final steps of integrin activation: the end game. Nat Rev Mol Cell Biol. 2010;11:288-300.

29. Ono A, Westein E, Hsiao S, Nesbitt WS, Hamilton JR, Schoenwaelder SM, Jackson SP. Identification of a fibrin-independent platelet contractile mechanism regulating primary hemostasis and thrombus growth. Blood. 2008;112:90-99.

30. Ninivaggi M, Feijge MA, Baaten CC, Kuiper GJ, Marcus MA, Ten Cate H, Lancé MD, Heemskerk JW, Van der Meijden PE. Additive roles of platelets and fibrinogen in whole-blood fibrin clot formation upon dilution as assessed by thromboelastometry. Thromb Haemost. 2014;111:447-457.

31. Schols SE, Feijge MA, Lancé MD, Hamulyák K, Ten Cate H, Heemskerk JW, Van Pampus EC. Effects of plasma dilution on tissue-factor-induced thrombin generation and thromboelastography: partly compensating role of platelets. Transfusion. 2008;48:2384-2394.

32. Castoldi E, Collins PW, Williamson PL, Bevers EM. Compound heterozygosity for 2 novel TMEM16F mutations in a patient with Scott syndrome. Blood. 2011;117:4399-4400.

33. Slaboch CL, Alber MS, Rosen ED, Ovaert TC. Mechano-rheological properties of the murine thrombus determined via nanoindentation and finite element modeling. J Mech Behav Biomed Mater. 2012;10:75-86.

34. Wolberg AS, Allen GA, Monroe DM, Hedner U, Roberts HR, Hoffman M. High dose factor VIla improves clot structure and stability in a model of hemophilia B. Br J Haematol. 2005;131:645-655.

35. Tomaiuolo M, Stalker TJ, Welsh JD, Diamond SL, Sinno T, Brass LF. A systems approach to hemostasis: 2. Computational analysis of molecular transport in the thrombus microenvironment. Blood. 2014;124:18161823.

36. Welsh JD, Stalker TJ, Voronov R, Muthard RW, Tomaiuolo M, Diamond SL, Brass LF. A systems approach to hemostasis: 1. The interdependence of thrombus architecture and agonist movements in the gaps between platelets. Blood. 2014;124:1808-1815.

37. Shen F, Kastrup CJ, Liu Y, Ismagilov RF. Threshold response of initiation of blood coagulation by tissue factor in patterned microfluidic capillaries is controlled by shear rate. Arterioscler Thromb Vasc Biol. 2008;28:2035-2041.

38. Kamocka MM, Mu J, Liu X, Chen N, Zollman A, Sturonas-Brown B, Dunn K, Xu Z, Chen DZ, Alber MS, Rosen ED. Two-photon intravital imaging of thrombus development. J Biomed Opt. 2010;15:016020.

39. Watts T, Barigou M, Nash GB. Comparative rheology of the adhesion of platelets and leukocytes from flowing blood: why are platelets so small? Am J Physiol Heart Circ Physiol. 2013;304:H1483-1494.

40. Ivanciu L, Krishnaswamy S, Camire RM. New insights into the spatiotemporal localization of prothrombinase in vivo. Blood. 2014;124:1705-1714.

41. Mastenbroek TG, Van Geffen JP, Heemskerk JW, Cosemans JM. Acute and persistent platelet and coagulant activities in atherothrombosis. J Thromb Haemost. 2015;13 Suppl 1:S272-280.

42. Stegner D, Nieswandt B. Platelet receptor signaling in thrombus formation. J Mol Med (Berl). 2011;89:109121. 


\section{Supplemental data}

\section{Methods}

\section{Healthy volunteers and patients}

Blood was taken from healthy volunteers and patients after informed consent was obtained in accordance with the Declaration of Helsinki. Studies were approved by the local medical ethics committee (METC 10-3-023). All donors were free from antiplatelet and anticoagulant medication for at least 2 weeks. Blood was taken from healthy control donors and from two patients with hemophilia B (both $5 \%$ factor IX), a patient with Scott syndrome (deficient anoctamin 6 expression), ${ }^{1}$ two patients with immune-induced thrombocytopenia (platelet count $85 \times 10^{9} / \mathrm{L}$ and $22 \times 10^{9} / \mathrm{L}$ ), or from three patients with dilutional coagulopathy due to massive fluid infusion during cardiothoracic surgery (4.5$5 \mathrm{~L}$ ), collected in the operating theatre. At least three blood samples were analyzed for all conditions.

\section{Blood collection and preparation}

Blood was collected in 1:10 (v/v) $3.2 \%$ trisodium citrate for whole blood perfusion experiments and the preparation of washed red blood cells, or in 1:6 (v/v) acidic citrate dextrose (ACD, $80 \mathrm{mM}$ trisodium citrate, $52 \mathrm{mM}$ citric acid and $180 \mathrm{mM}$ glucose) for the isolation of washed platelets. Platelets and red blood cells were isolated as described. ${ }^{2}$ Cell count was determined using a thrombocounter (Coulter Electronics, High Wycombe, United Kingdom).

\section{Formation of platelet-fibrin thrombi under conditions of coagulation and flow}

Thrombus formation under flow conditions was determined by perfusion of citrated whole blood over a collagen/tissue factor (TF) coated glass coverslip in a transparent parallel-plate perfusion chamber (width $3 \mathrm{~mm}$, depth $50 \mu \mathrm{m}$, length $300 \mathrm{~mm}$ ). ${ }^{3}$ Coverslips were coated with microspots containing 20 or $100 \mathrm{ng}$ Horm type I collagen (Nycomed Pharma, Munich, Germany) in the absence or presence of $10 \mathrm{pg}$ recombinant human TF (Innovin; Dade Behring, Deerfield IL, USA). After coating, surfaces were blocked with $1 \%$ bovine serum albumin (BSA; Sigma, St. Louis MO, USA) in Hepes buffer pH 7.45 (10 mM Hepes, $136 \mathrm{mM} \mathrm{NaCl}, 2.7 \mathrm{mM} \mathrm{KCl}, 2 \mathrm{mM} \mathrm{MgCl}$ ), and used within 4 hours. Immediately before use, blood samples were pre-labeled with the membrane probe $\mathrm{DiOC}_{6}$ (f.c. 0.5 $\mu \mathrm{g} / \mathrm{mL}$, Anaspec, Reeuwijk, The Netherlands) to identify platelets (green) and with Alexa fluor (AF)647-fibrinogen (f.c. $16.5 \mathrm{\mu g} / \mathrm{mL}$, Molecular Probes, Life Technologies, New York NY, USA) to detect fibrinogen and fibrin (red). Using two pulse-free micro-pumps (Model 11 Plus, 70-2212, Harvard apparatus, Holliston MA, USA) and a Y-shaped flattened mixing tube, blood samples in $1 \mathrm{~mL}$ plastic syringe (Becton Dickinson, Franklin Lakes NJ, USA) were mixed with coagulation medium (Hepes buffer $\mathrm{pH} 7.45$ supplemented with $32 \mathrm{mM}$ $\mathrm{MgCl}_{2}$ and $63 \mathrm{mM} \mathrm{CaCl}$ ) also in $1 \mathrm{~mL}$ syringe at a volume ratio of 10:1 (push mode). This resulted in complete mixing and physiological (mM) concentrations of $\mathrm{Ca}^{2+}$ and $\mathrm{Mg}^{2+}$. 
Where indicated, blood samples were pre-treated with $3 \mu \mathrm{g} / \mathrm{mL}$ hirudin (Refludan; Schering, Berlin, Germany) to block formed thrombin. Blood perfusion over microspots in the flow chamber was at a wall shear rate of 150 or $1000 \mathrm{~s}^{-1}$ for at least 7 minutes.

For assessing platelet contraction in a thrombus, citrated blood samples were spiked with $3 \%$ of autologous, washed DiOC $_{6}$-labeled platelets. Where indicated, spiked blood samples were pre-treated with Gly-Pro-Arg-Pro (GPRP) or hirudin.

\section{Two-color confocal fluorescence microscopy and image analysis}

During or after blood perfusion, two-colored images were recorded using a fast line-scanning Zeiss LSM7 system, equipped with OPSS lasers and a 40x oil-immersion objective (numerical aperture 1.30) (Carl Zeiss, Oberkochen, Germany). Confocal fluorescence images (16 bit, $1024 \times 1024$ pixels, single channel plus overlay) were obtained at both 488 and $635 \mathrm{~nm}$ excitation. Fluorescence was detected with an ultrasensitive CCD line camera after selection by optical emission filters of 495-555 nm and 665-750 nm (pinhole $1 \mathrm{AU}$ ). For time series, fluorescence images were taken at $1 \mathrm{~s}$ intervals; for z stacks, fluorescence images were taken at $1 \mu \mathrm{m}$ z-steps.

Image analysis of morphometry and fluorescence was performed with Live7 Metamorph software, version 7.5.0.0 (MDS Analytical Technologies, Sunnyvale, Canada) and ImageJ (version 1.48g: Rasband, NIH, Bethesda, MD, USA), as described elsewhere.5 For the quantification of fibrin and platelet fluorescence, images were thresholded to eliminate background. Integrated fluorescence intensity as well as percentage area covered were calculated per image. For co-localization of two-color fluorescence, binary images were created based on threshold to determine overlap percentages. As a standard, integrated fluorescence intensity was compared to surface area coverage of fluorescence features. Considering the overall similarity of these two analyses (Supplemental Fig. 1), for most conditions data of surface area coverage are given only.

For the quantification of (fluorescent) fibrin formation, threshold levels of fibrinogen binding to platelets was determined under conditions where coagulation was inhibited. Image intensities above this threshold were considered as originating from fibrin fibers. The presence of fluorescent fibrin fibers was always confirmed from brightfield images. Times to onset of fibrin formation were the moments at which the fluorescence threshold was passed, as confirmed by visual inspection of brightfield images.

For measurement of platelet contraction in a thrombus, time series of $\mathrm{DiOC}_{6}{ }^{-}$ labeled images were collected during flow. Movement towards each other of labeled platelets within thrombi was analyzed off-line via tracking analysis, as described. ${ }^{6}$ In brief, individual images of time-lapse series were thresholded to a binary images. Using the Mtrack2 plugin displacement was measured by including objects (platelets) of $<5$ 
pixels which were present $>60$ seconds. Objects were rejected when the displacement was $>25$ pixels s ${ }^{-1}$ (indicating noise or platelets not part of one thrombus). Calculated was per condition the average movement of platelets in time $(\mu \mathrm{m} / \mathrm{s})$.

\section{Dilution and reconstitution of whole blood samples}

Citrated blood was diluted in vitro with saline medium, consisting of $154 \mathrm{mM}$ $\mathrm{NaCl}, 10.8 \mathrm{mM}$ trisodium citrate, $2 \mathrm{mM} \mathrm{CaCl}_{2}$ and $2 \mathrm{mM} \mathrm{MgCl}_{2}$, in order to keep equal concentrations of free $\mathrm{Ca}^{2+}$ and $\mathrm{Mg}^{2+}$ in all diluted samples. ${ }^{2}$ Washed platelets and/or red cells were added to diluted blood samples to restore original cell counts, as described before. ${ }^{2}$ Amounts of fluorescent labels, $\mathrm{DiOC}_{6}$ and AF647-fibrinogen, were adjusted according to the extent of dilution and reconstitution.

\section{Measurement of thrombin generation on microspots in flow chamber}

Fluorogenic thrombin substrate Z-Gly-Gly-Arg-AMC (f.c. $0.5 \mathrm{mM}$, Thrombinoscope, Maastricht, The Netherlands) was added to blood samples where indicated, and used to measure thrombin generation on preformed thrombi under static conditions. ${ }^{7}$ Realtime fluorescence accumulation due to substrate cleavage was recorded at 447/30 $\mathrm{nm}$ emission every 30 seconds for at least 5 minutes, using an inverted fluorescence microscope equipped with an Olympus $60 \times$ oil-immersion objective (numerical aperture 1.35), equipped with a $360 \mathrm{~nm}$ led cube (EVOS, Life Technologies, Bleiswijk, The Netherlands). ${ }^{5}$ Temporal analysis of fluorescence was performed using ImageJ.

\section{Determination of microelasticity of thrombi on microspots by nanoindentation technology}

Microelasticity of formed thrombi on coverslips was analyzed with a recently developed Piuma nanoindenter (Optics11, Amsterdam, The Netherlands), which features a calibrated nanoindentation probe consisting of a cantilever fabricated on top of a ferruled optical fibre. Recent developments in indentation probe fabrication have resulted in probes that can measure soft (biological) materials, e.g. cartilage and vascular models. These probes measure the material hardness in the Pascal to kiloPascal range. ${ }^{8}$ In atomic force microscopy a cantilever tip is used that is repelled from the surface by minute forces. In contrast, in nanoindentation methods a spherical, cylinder or Berkovich shaped tip is used that is forced into the sample. The employed Optics11 Piuma Nanoindenter system has been validated before with biological samples. ${ }^{9-11}$

In brief, a probe with a defined cantilever of $1.39 \mathrm{~N} / \mathrm{m}$ and calibrated spherical tip of $56.5 \mu \mathrm{m}$ was gradually brought into contact with the sample surface, up to a depth of maximum $10 \%$ of the sample thickness. ${ }^{12}$ The counter-force of the sample on the tip results in cantilever bending, which is linear with the load on the cantilever. The result is a load-displacement curve of both loading (indenting) and unloading (retraction) of the probe. The probe, fixed on a closed-loop z-piezoelectric translator, was equipped with an integrated strain gage sensor and controlled by a closed-loop controller. From the slope of the unloading curve, the maximum load and the indentation area (tip surface area), a reduced Young's modulus (RedYM) can be calculated as a proxy measure of microelasticity. ${ }^{13}$ The modulus is the amount of force per unit area (stress), needed to 
reach a certain amount of deformation (unit $=\mathrm{Pa}$ ). Hence, a higher RedYM indicates that the given material is harder to deform. This measurement outcome is similar to atomic force microscopy. ${ }^{14}$

As a conservative rule of thumb, the indentation value is independent of the height and size of a sample, provided that the indentation depth is smaller than the sample radius and $<10 \%$ of the sample height. ${ }^{12,15}$ With these criterions met for the present conditions, the RedYM reflects the elastic modulus of platelet-fibrin thrombi. The attractiveness of the nanoindentation technique is that the mechanoelastic properties of a microscopic sample can directly be determined without any further sample knowledge. A drawback is the lack of visual feedback of the probe location above the thrombus sample. To overcome this, we have indented all samples (old and new conditions) 36 times in a grid-like pattern. With an optical magnifier, we further confirmed that the right spots on the sample were indented.

Coverslips with thrombi were carefully washed and incubated with Hepes buffer $\mathrm{pH}$ 7.45. The diameter of the contact area of indentation tip with thrombi was $\sim 20 \mu \mathrm{m}^{2}$. Per microspot, $6 \times 6$ indentations were performed following a gridding pattern with a stepsize of $50 \mu \mathrm{m}$. All indents were depth controlled ( $1 \mu \mathrm{m}$ to a maximum of $5 \mu \mathrm{m})$. Loading and unloading times were set at 3 seconds, data were collected for 1 second per step. The unloading curves were used to calculate slopes using the compliance method of Oliver and Pharr in order to calculate the RedYM. ${ }^{13,16}$ This is a measure of the microrigidity of the indented spot, which is inversely related to the microelasticity. A high RedYM reflects high microrigidity or stiffness of the indented sample and, hence, low microelasticity. Of the grid nanoindentations, only curves reflecting thrombi on glass were included. As negative and positive controls bare collagen surfaces and macroscopic fibrin clots were used, which showed a low and high microelasticity, respectively. Furthermore, scanning electron microscopy pictures were obtained for direct comparison of the dimensions of tip and thrombi.

\section{Scanning electron microscopy}

Thrombi were formed on microspots in a perfusion chamber, as described above, and prepared for scanning electron microscopy. In short, after disassembly of the perfusion chamber, coverslips were removed and gently washed with Hepes buffer $\mathrm{pH}$ 7.45. Fixation was with $2.5 \%$ glutaraldehyde in $0.1 \mathrm{M} \mathrm{K}$-phosphate buffer $\mathrm{pH} 7.4$ at $4{ }^{\circ} \mathrm{C}$. Following a rinsing step with $0.1 \mathrm{M}$ phosphate buffer, samples were dehydrated in graded ethanol series, then critical point dried, mounted with silver paint on specimen stubs, and coated with gold. Thrombi were visualised with a Philips XL30 scanning electron microscope at $10 \mathrm{kV}$ (Eindhoven, The Netherlands).

\section{Statistics}

Statistical significance of differences between two independent groups was determined using the independent samples $t$ test. Differences with $p$ values $<0.05$ were considered significant. Error bars indicate inter-individual variability, except for patient data, where the variation of independent measurements is shown; values outside reference ranges of controls (mean $\pm 2 \mathrm{SD}$ ) were considered to be different. 
A

Platelets

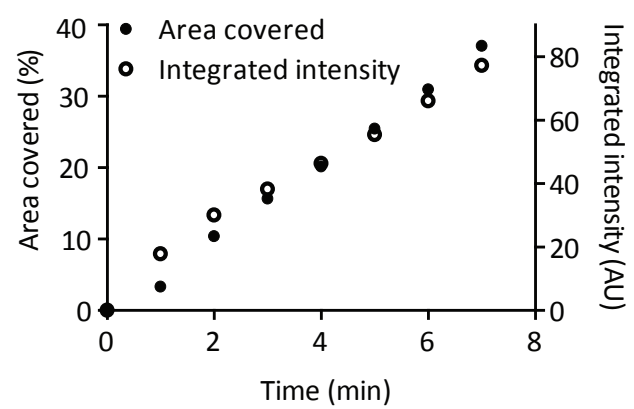

B

Fibrin

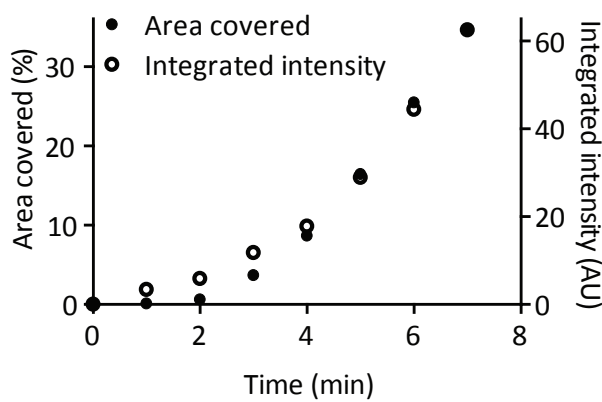

Supplemental Figure 1. Quantification of platelet-fibrin thrombus formation on collagen/TF surfaces. Citrated whole blood was perfused during recalcification over microspots with collagen (100 ng) and TF $(10 \mathrm{pg})$ for 7 minutes at a wall shear rate of $1000 \mathrm{~s}^{-1}$. Blood samples were pretreated with $\mathrm{DiOC}_{6}$ to label platelets (green) and with AF647-fibrinogen (red). (A, B) Analysis of integrated fluorescence intensity (open) and surface area coverage above background (close) from deposited platelets (A) and fibrin (B). Note high similarity of either analysis parameter.

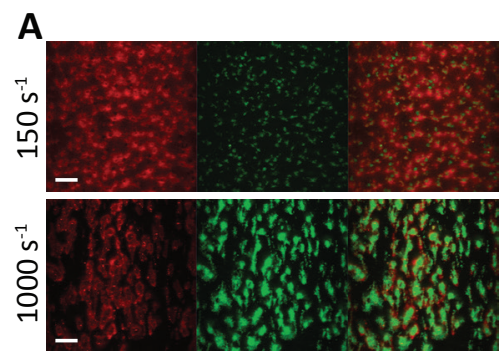

B

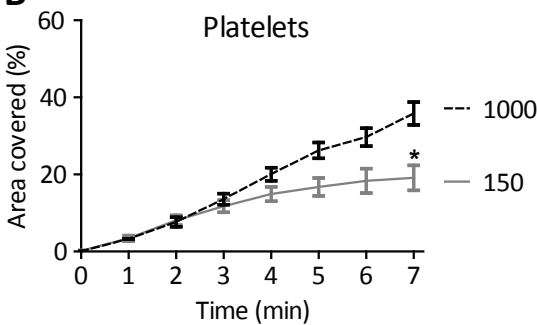

C
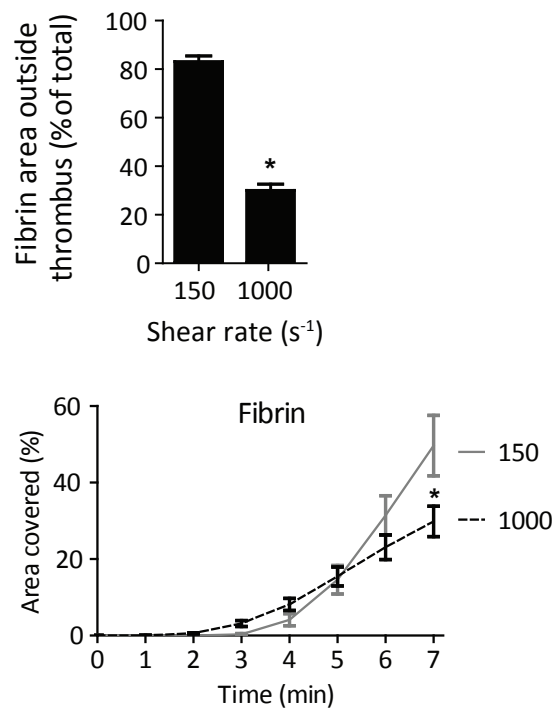

Supplemental Figure 2. Effect of wall shear rate in platelet-fibrin thrombus formation on collagen-tissue factor surfaces. Platelet-fibrin thrombi were formed by blood perfusion over microspots with collagen (100 $\mathrm{ng})$ and TF (10 pg), as in Figure 1. Wall shear rate was 150 or $1000 \mathrm{~s}^{-1}$. (A) Representative images after 7 minutes of flow of $\mathrm{DiOC}_{6}$-labeled platelets (green) and AF647-fibrin(ogen) (red) (bars, $\left.50 \mu \mathrm{m}\right)$. (B) Timedependent deposition of fluorescent-labeled platelets and fibrin during blood perfusion. (C) Quantification of platelet-fibrin co-localization at indicated shear rates. Means \pm SEM $(n=5-10),{ }^{*} p<0.05$. 

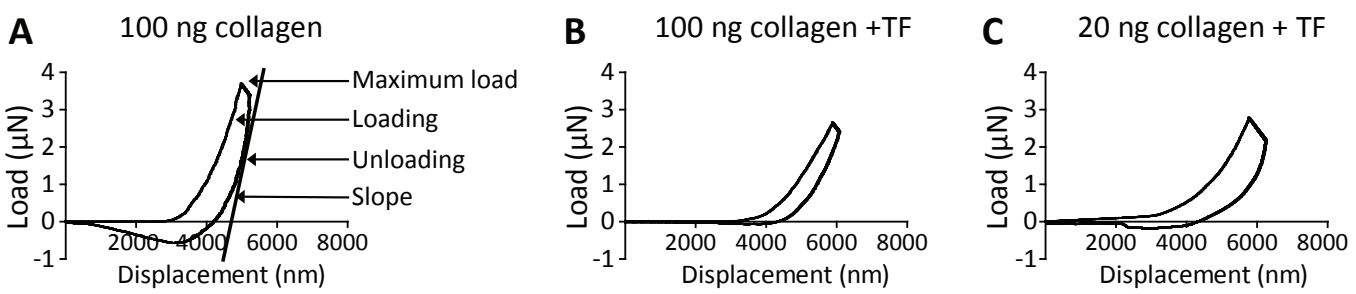

Supplemental Figure 3. Nanoindentation load-displacement curves for the assessment of microelasticity of platelet-fibrin thrombi. Platelet-fibrin thrombi were formed by blood perfusion over microspots with collagen $\pm T F$, as in Figure 1. Microspot surfaces were subjected to nanoindentation, as in Figure 4. Shown are representative load-displacement curves for thrombi formed on (A) $100 \mathrm{ng}$ collagen, (B) $100 \mathrm{ng}$ collagen plus $10 \mathrm{pg}$ TF, or (C) $20 \mathrm{ng}$ collagen plus $10 \mathrm{pg}$ TF. Indicated is the loading curve during indentation, the maximum load and the unloading curve with slope (= RedYM). The RedYM represents a measure of the microrigidity of the sample, which is inversely related to microelasticity.

A

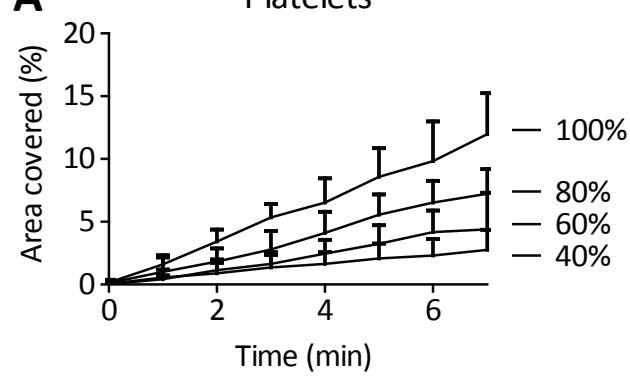

B

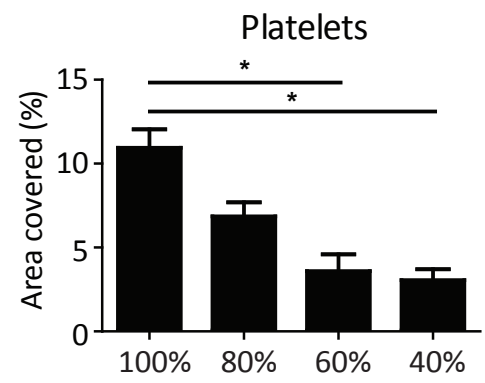

Fibrin

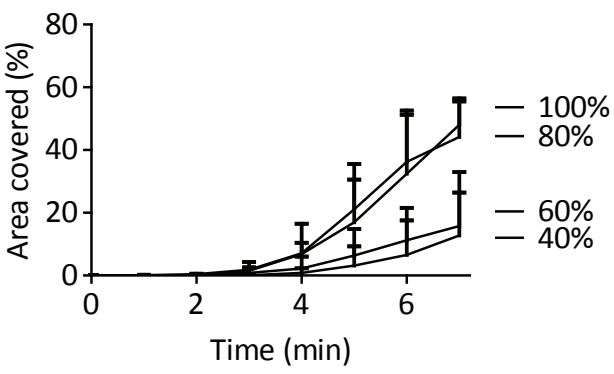

Fibrin

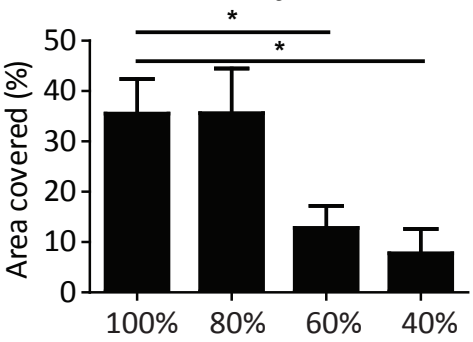

Supplemental Figure 4. Effects of blood dilution on formation of platelet-fibrin thrombus at low shear rate. Platelet-fibrin thrombi were formed by perfusion of blood over microspots with collagen (100 ng) and TF (10 $\mathrm{pg}$ ) at low shear rate of $150 \mathrm{~s}^{-1}$ for 7 minutes. Whole blood samples were undiluted or diluted to indicated \% with saline (see Figure 5). (A) Time-dependent accumulation of fluoresence from platelets (green) and fibrin (red) during blood perfusion. (B) Fluoresence area covered by platelets and fibrin after 7 minutes of flow. Means $\pm \operatorname{SEM}(n=6),{ }^{*} p<0.05$. 

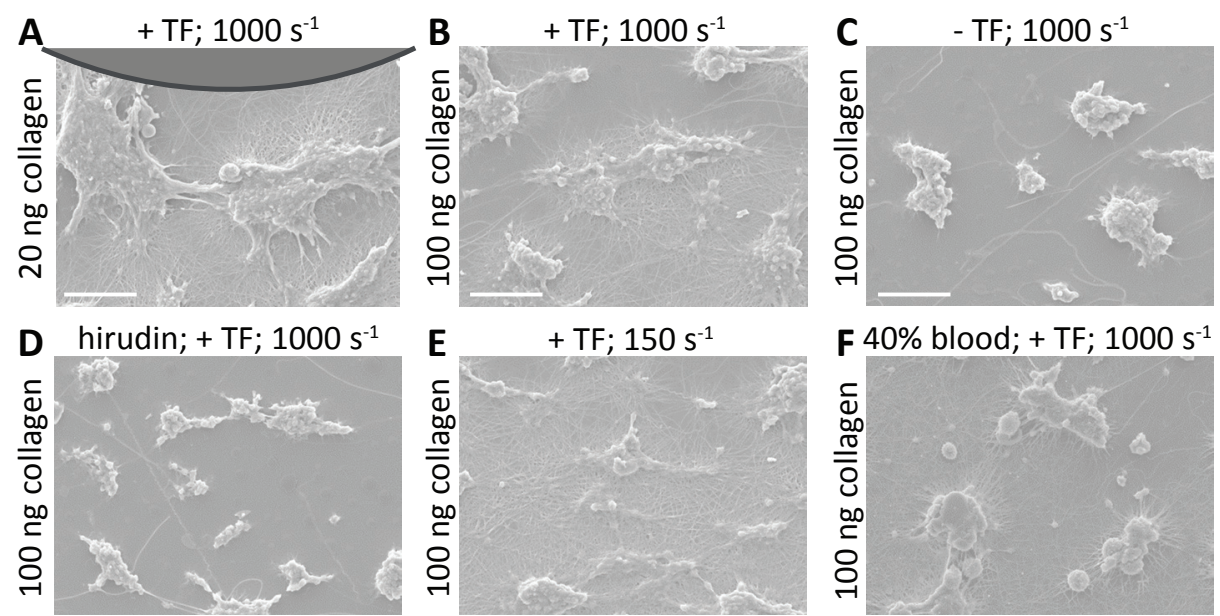

Supplemental Figure 5. Scanning electron microscopy of platelet-fibrin thrombus formed on different surfaces. Platelet-fibrin thrombi were formed by perfusion of blood over microspots with collagen \pm TF at shear of 150 or $1000 \mathrm{~s}^{-1}$. Thrombi on microspots were fixed, stained and visualised with a scanning electron microscope at $10 \mathrm{kV}$. Representative $(n=3)$ images are shown from thrombi formed on: (A) $20 \mathrm{ng}$ collagen + TF; (B) 100 ng collagen + TF; (C) 100 ng collagen; (D) 100 ng collagen + TF, with hirudin in blood; (E) 100 ng collagen $+\mathrm{TF}$, perfusion at $150 \mathrm{~s}^{-1}$; (F) $100 \mathrm{ng}$ collagen $+\mathrm{TF}, 40 \%$ whole blood (bars, $20 \mu \mathrm{m}$ ). Upper part shows schematic representation of the spherical nanoindentation cantilever to scale. 
Supplemental Table 1. Collagen-tissue factor surface determines platelet-fibrin thrombus buildup under flow. Citrated whole blood was perfused during recalcification over microspots with collagen (20 or $100 \mathrm{ng}$ ) with(out) TF (10 pg) for 7 minutes at a wall shear rate of 150 or $1000 \mathrm{~s}^{-1}$. Blood samples were pretreated with $\mathrm{DiOC}_{6}$ to label platelets and with AF647-fibrinogen to monitor fibrin formation. Two-color confocal microscopic images were recorded in real time at a frequency of $1 \mathrm{~Hz}$. For determination of thrombus volume, confocal $z$ stacks were recorded after 7 minutes of perfusion (z-step $1 \mu \mathrm{m})$. *SAC, surface area covered; NA, not applicable due to relatively low thrombus height. Means \pm SEM ( $n=6-15)$.

\begin{tabular}{|c|c|c|c|c|c|c|c|}
\hline & & \multicolumn{2}{|c|}{ Collagen 100} & \multicolumn{2}{|c|}{ Collagen $100+\mathrm{TF}$} & \multicolumn{2}{|c|}{ Collagen $20+T F$} \\
\hline & & $150 \mathrm{~s}^{-1}$ & $1000 s^{-1}$ & $150 s^{-1}$ & $1000 \mathrm{~s}^{-1}$ & $150 \mathrm{~s}^{-1}$ & $1000 s^{-1}$ \\
\hline \multirow[t]{2}{*}{ SAC* (\%) } & platelets & $9.04 \pm 1.7$ & $27.0 \pm 2.2$ & $19.1 \pm 3.2$ & $33.8 \pm 3.0$ & $5.62 \pm 0.7$ & $12.3 \pm 1.4$ \\
\hline & fibrin & $0.73 \pm 0.7$ & $1.19 \pm 0.1$ & $49.9 \pm 6.8$ & $29.8 \pm 4.0$ & $4.30 \pm 1.7$ & $5.76 \pm 1.1$ \\
\hline \multirow{3}{*}{$\begin{array}{l}\text { Fibrin } \\
\text { area } \\
\text { outside } \\
\text { thrombus } \\
\text { (\% of } \\
\text { total) }\end{array}$} & $\begin{array}{l}0-10 \mu \mathrm{m} \\
\text { (base) }\end{array}$ & $48.8 \pm 9.7$ & $36.3 \pm 8.4$ & $83.1 \pm 0.3$ & $28.8 \pm 1.9$ & $69.6 \pm 3.0$ & $9.7 \pm 2.8$ \\
\hline & $\begin{array}{l}10-20 \mu \mathrm{m} \\
\text { (center) }\end{array}$ & $54.0 \pm 18.0$ & $55.5 \pm 8.2$ & $91.4 \pm 2.1$ & $8.9 \pm 2.1$ & $55.8 \pm 5.4$ & $2.3 \pm 0.6$ \\
\hline & $\begin{array}{l}>20 \mu \mathrm{m} \\
\text { (top) }\end{array}$ & $54.1 \pm 13.9$ & $75.5 \pm 14.0$ & $76.9 \pm 4.8$ & $5.3 \pm 1.8$ & $57.5 \pm 1.3$ & $5.2 \pm 1.1$ \\
\hline \multirow{3}{*}{$\begin{array}{l}\text { Thrombus } \\
\text { volume } \\
\left(\times 10^{6}\right. \\
\left.\mu \mathrm{m}^{3}\right)\end{array}$} & platelets & $0.15 \pm 0.02$ & $0.28 \pm 0.04$ & $0.16 \pm 0.02$ & $0.79 \pm 0.1$ & $0.15 \pm 0.02$ & $0.40 \pm 0.1$ \\
\hline & fibrin & $0.03 \pm 0.007$ & $0.02 \pm 0.01$ & $0.72 \pm 0.03$ & $0.76 \pm 0.2$ & $0.20 \pm 0.03$ & $0.24 \pm 0.07$ \\
\hline & total & $0.16 \pm 0.02$ & $0.28 \pm 0.04$ & $0.78 \pm 0.02$ & $1.10 \pm 0.2$ & $0.29 \pm 0.04$ & $0.46 \pm 0.1$ \\
\hline \multirow{3}{*}{$\begin{array}{l}\text { Ratio } \\
\text { fibrin/ } \\
\text { platelets }\end{array}$} & $\begin{array}{l}0-10 \mu \mathrm{m} \\
\text { (base) }\end{array}$ & $0.86 \pm 0.07$ & $0.84 \pm 0.02$ & $0.97 \pm 0.02$ & $1.21 \pm 0.1$ & $0.88 \pm 0.01$ & $0.97 \pm 0.1$ \\
\hline & $\begin{array}{l}10-20 \mu \mathrm{m} \\
\text { (center) }\end{array}$ & $1.09 \pm 0.1$ & $1.20 \pm 0.1$ & $1.07 \pm 0.02$ & $0.87 \pm 0.04$ & $0.98 \pm 0.01$ & $0.93 \pm 0.08$ \\
\hline & $\begin{array}{l}>20 \mu \mathrm{m} \\
\text { (top) }\end{array}$ & $N A$ & NA & $N A$ & $1.07 \pm 0.1$ & $1.18 \pm 0.01$ & $1.27 \pm 0.1$ \\
\hline
\end{tabular}




\section{References}

1. Castoldi E, Collins PW, Williamson PL, Bevers EM. Compound heterozygosity for 2 novel TMEM16F mutations in a patient with Scott syndrome. Blood. 2011;117:4399-4400.

2. Ninivaggi M, Feijge MA, Baaten CC, Kuiper GJ, Marcus MA, Ten Cate H, Lancé MD, Heemskerk JW, Van der Meijden PE. Additive roles of platelets and fibrinogen in whole-blood fibrin clot formation upon dilution as assessed by thromboelastometry. Thromb Haemost. 2014;111:447-457.

3. Van Kruchten R, Cosemans JM, Heemskerk JW. Measurement of whole blood thrombus formation using parallel-plate flow chambers - a practical guide. Platelets. 2012;23:229-242.

4. Gilio K, Van Kruchten R, Braun A, Berna-Erro A, Feijge MA, Stegner D, Van der Meijden PE, Kuijpers MJ, Varga-Szabo D, Heemskerk JW, Nieswandt B. Roles of platelet STIM1 and Orai1 in glycoprotein VI- and thrombin-dependent procoagulant activity and thrombus formation. J Biol Chem. 2010;285:23629-23638.

5. De Witt SM, Swieringa F, Cavill R, Lamers MM, Van Kruchten R, Mastenbroek T, Baaten C, Coort S, Pugh N, Schulz A, Scharrer I, Jurk K, Zieger B, Clemetson KJ, Farndale RW, Heemskerk JW, Cosemans JM. Identification of platelet function defects by multi-parameter assessment of thrombus formation. Nat Commun. 2014;5:4257.

6. Smal I, Meijering E. Quantitative comparison of multiframe data association techniques for particle tracking in time-lapse fluorescence microscopy. Med Image Anal. 2015;24:163-189.

7. Berny MA, Munnix IC, Auger JM, Schols SE, Cosemans JM, Panizzi P, Bock PE, Watson SP, McCarty OJ, Heemskerk JW. Spatial distribution of factor Xa, thrombin, and fibrin(ogen) on thrombi at venous shear. PLoS One. 2010;5:e10415.

8. Chavan D, Van de Watering TC, Gruca G, Rector JH, Heeck K, Slaman M, lannuzzi D. Ferrule-top nanoindenter: an optomechanical fiber sensor for nanoindentation. Rev Sci Instrum. 2012;83:115110.

9. Mattei G, Gruca G, Rijnveld N, Ahluwalia A. The nano-epsilon dot method for strain rate viscoelastic characterisation of soft biomaterials by spherical nano-indentation. J Mech Behav Biomed Mater. 2015;50:150-159.

10. Neufurth M, Wang X, Tolba E, Dorweiler B, Schroder HC, Link T, Diehl-Seifert B, Muller WE. Modular Small Diameter Vascular Grafts with Bioactive Functionalities. PLoS One. 2015;10:e0133632.

11. Wang S, Wang X, Draenert FG, Albert O, Schroder HC, Mailander V, Mitov G, Muller WE. Bioactive and biodegradable silica biomaterial for bone regeneration. Bone. 2014;67:292-304.

12. Panich N, Sun Y. Effect of penetration depth on indentation response of soft coatings on hard substrates: a finite element analysis. Surface \& Coatings Technology. 2004;182:342-350.

13. Oliver WC, Pharr GM. An improved technique for determining hardness and elastic modulus using load and displacement sensing indentation experiments. J Mater Res. 1992;7:1564-1583.

14. Chavan D, Andres D, lannuzzi D. Note: ferrule-top atomic force microscope. II. Imaging in tapping mode and at low temperature. Rev Sci Instrum. 2011;82:046107.

15. Poon B, Rittel D, Ravichandran G. An analysis of nanoindentation in elasto-plastic solids. Int J Solids Struct. 2008;45:6399-6415.

16. Cabibbo M, Ricci P, Cecchini R, Rymuza Z, Sullivan J, Dub S, Cohen S. An international round-robin calibration protocol for nanoindentation measurements. Micron. 2012;43:215-222. 




\section{Chapter 5}

Platelet heterogeneity in glycoprotein shedding: consequence for procoagulant responsiveness

Baaten CC, Swieringa F, Misztal T, Mastenbroek TG, Feijge MA, Bock PE, Donners MM, Collins PW, Li R, van der Meijden PE, Heemskerk JW

Blood Advances. 2018; major revision 


\section{Abstract}

The platelet receptors glycoprotein (GP)Iba and GPVI are known to be cleaved by members of the family of A Disintegrin And Metalloproteases, ADAM10 and ADAM17, but the mechanisms and signaling pathways underlying this shedding are not well understood. Here, we disclosed that: (i) glycoprotein shedding is confined to distinct platelet populations showing near complete shedding, and (ii) the heterogeneity between platelets is independent of agonist type, but associated with phosphatidylserine (PS) exposure. We identified three distinct pathways of shedding, (iii) induced by elevated $\mathrm{Ca}^{2+}$, protein kinase $\mathrm{C}$ (PKC) or caspase activation. Furthermore, we noted that: (iv) receptor shedding enhances platelet procoagulant activity in terms of increased coagulation factor binding and fibrin formation. In response to $\mathrm{Ca}^{2+}$-rising agents, shedding of GPIb $\alpha$ was abolished by ADAM10/17 inhibition, but not by blockage of calpain. Stimulation of PKC caused shedding of only GPIba, which was annulled by a kinase inhibitor. In response to the pro-apoptotic agent ABT-737 only, glycoprotein shedding was abolished by caspase inhibition. Surprisingly, in Scott syndrome platelets, deficient in $\mathrm{Ca}^{2+}$-dependent PS exposure, shedding occurred normally. In whole blood thrombus formation, ADAM-dependent shedding of GPIba and GPVI stimulated fibrin formation at those platelets showing $\mathrm{Ca}^{2+}$-dependent ballooning and PS exposure. In conclusion, these novel findings indicate that GPIba and GPVI shedding is linked to high $\mathrm{Ca}^{2+}$ elevation or caspase activation, occurs independently of PS exposure, and enhances platelet coagulant activity.

Visual abstract

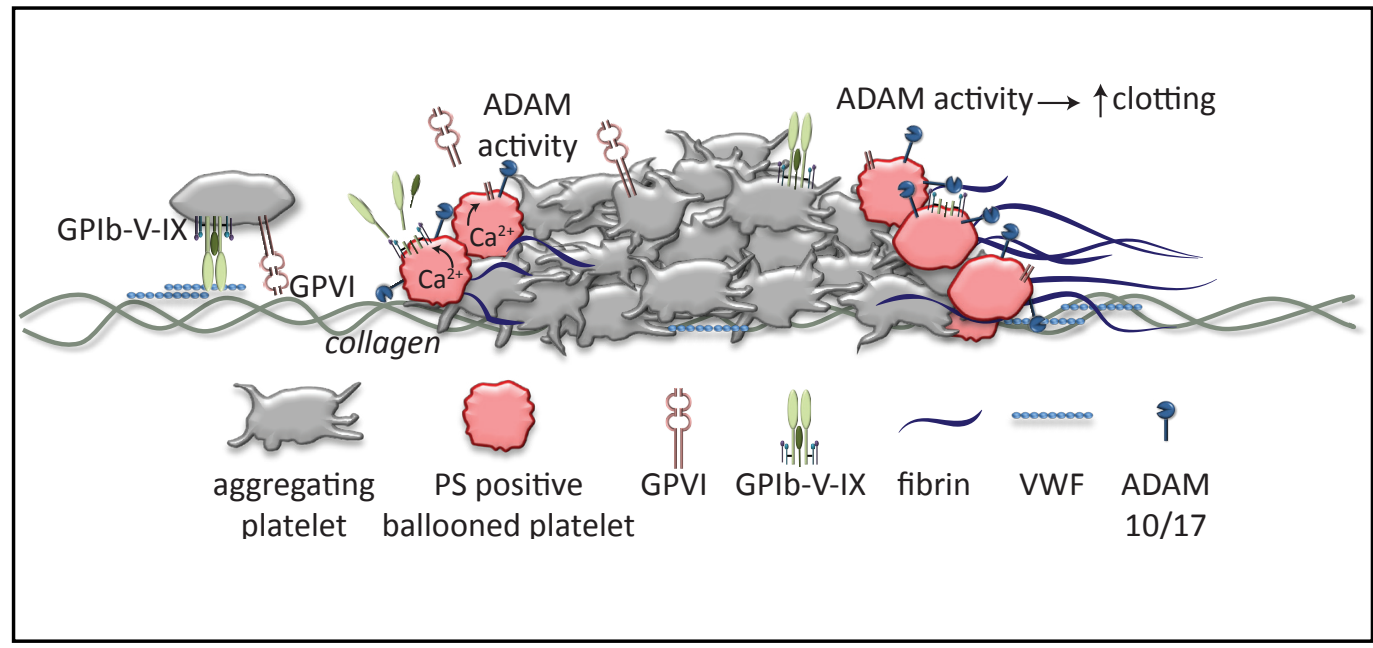




\section{Introduction}

During the processes of hemostasis and thrombosis, blood platelets are triggered to adhere to an injured or atherosclerotic vessel wall. Flow-dependent platelet adhesion and subsequent aggregate formation are controlled, in a synergistic way, by multiple glycoprotein receptors. ${ }^{1-3}$ Two crucial adhesive receptors implicated in platelet adhesion and aggregation are glycoprotein VI (GPVI), interacting with collagen and fibrin, and GPIb-V-IX, binding primarily to von Willebrand factor (VWF). Other receptors with highadhesive strength are CLEC-2 with unknown ligands in the healthy vessel wall, integrin $\alpha_{6} \beta_{1}$ binding laminin, integrin $\alpha_{11 b} \beta_{3}$ binding e.g. fibrinogen, and integrin $\alpha_{2} \beta_{1}$ also interacting with collagen. ${ }^{4}$ Research in the last years has indicated that, in activated platelets, most and perhaps all of these adhesive receptors can be switched or cleaved off, which points to the presence of post-activation mechanisms to control platelet-substrate interaction. Hence, platelets with high cytosolic $\mathrm{Ca}^{2+}$ levels and phosphatidylserine (PS) exposure undergo calpain-dependent cleavage of the intracellular integrin $\beta_{3}$ chain and integrinlinked proteins, resulting in inactivation of $\alpha_{\| 1} \beta_{3}{ }^{5,6}$ In addition, in platelets stimulated with various agonists, the extracellular domains of GPIb $\alpha$ and GPVI can be cleaved off. Studies with inhibitors and murine knockouts have indicated that the latter cleavage is mediated by two members of the A Disintegrin And Metalloprotease (ADAM) family. GPIb $\alpha$ appears to be mainly cleaved by ADAM17, whilst GPVI shedding is predominantly controlled by ADAM10. ${ }^{7-10}$ However, significant functional redundancy exists between these two ADAM isoforms. ${ }^{11}$

Several researchers have reported that extracellular domains of GPIba and GPVI can be cleaved or shed in response to different platelet-stimulating agents. These include collagen and thrombin, the protein kinase $C$ (PKC) stimulus phorbol myristate acetate (PMA), mitochondrial uncoupling compounds and apoptosis-inducing agents. ${ }^{7,9,11-13}$ Shedding of GPIba is furthermore triggered by high wall shear rates or by platelet storage. ${ }^{14,15}$ Unclear however are the precise signaling pathways causing glycoprotein shedding. An early concept, based on the use of the compound W7, suggested that ADAM activity and shedding are negatively regulated by the $\mathrm{Ca}^{2+}$-dependent protein kinase cofactor, calmodulin. ${ }^{8,9}$ Other reports propose that reactive oxygen species (ROS) produced in the mitochondria trigger ADAM activation and hence shedding. ${ }^{12,16,17}$ Furthermore, in lymphocytes receptor shedding was found to be dependent on phospholipid scrambling and PS exposure, which are key processes in apoptosis. ${ }^{18}$ Together, this points to the presence of one or more mechanisms of ADAM-dependent receptor shedding, occurring in highly activated or apoptotic cells that serve to down-regulate adhesiveness.

Here, we hypothesized that, in human platelets, four distinct pathways can contribute to ADAM-mediated shedding of GPIba and GPVI, i.e. $\mathrm{Ca}^{2+}$ elevation, PKC activation, PS exposure and caspase activity. We studied the involvement of these pathways in populations of platelets stimulated with a broad range of postulated sheddinginducing agents. The results indicate that shedding is regulated the level of the single platelet primarily via $\mathrm{Ca}^{2+}$ - or caspase-dependent pathways that are accompanied by, but 
not caused by PS exposure. Furthermore, our data show that the population of platelets with shed receptors shows an increased procoagulant activity, thus compensating for a reduced adhesiveness.

\section{Materials and methods}

\section{Materials}

For materials, see supplements.

\section{Blood collection and platelet preparation}

Human blood was obtained from healthy volunteers and a patient with Scott syndrome after informed consent in accordance with the Declaration of Helsinki, under protocols reviewed by the local ethics committee. The Scott patient was genotyped as compound heterozygous in ANO6 with one mutation, IVS $6+1 \mathrm{G} \rightarrow \mathrm{A}$, resulting in exon 6 skipping, and a second mutation (c.1219insT) causing a premature stop in translation. ${ }^{19}$

Blood was collected into $3.2 \%$ citrate for flow perfusion studies, or into acid-citrate dextrose (1:6 ACD, $80 \mathrm{mM}$ trisodium citrate, $52 \mathrm{mM}$ citric acid and $180 \mathrm{mM}$ glucose) for platelet isolation. The first $3 \mathrm{~mL}$ of blood were discarded. Washed platelets were prepared as described before. ${ }^{20}$ Platelet count was determined with a Sysmex XP300 thrombocounter (Sysmex, Chuo-ku Kobe, Japan).

\section{Flow cytometric analyses}

Pre-incubations of washed platelets $\left(1 \times 10^{8} / \mathrm{mL}\right)$ with inhibitor or vehicle were performed for $15 \mathrm{~min}$ at $37{ }^{\circ} \mathrm{C}$. After addition of $2 \mathrm{mM} \mathrm{CaCl}{ }_{2}$, cells were stimulated for indicated times with (combinations of) the following agonists at near-saturating doses: ABT-737 $(10 \mu \mathrm{M})$, ionomycin $(10 \mu \mathrm{M})$, convulxin $(100 \mathrm{ng} / \mathrm{mL}), \mathrm{CRP}-\mathrm{XL}(5 \mu \mathrm{g} / \mathrm{mL})$, thrombin $(4 \mathrm{nM})$, SFLLRN $(15 \mu \mathrm{M}), 2 \mathrm{MeS}-\mathrm{ADP}(10 \mu \mathrm{M}), \mathrm{CCCP}(100 \mu \mathrm{M})$ or PMA (100 nM). Surface expression of GPIba, GPVI or GPIX was measured in subsamples, after labeling with FITC anti-GPIba mAb $(10 \mu \mathrm{g} / \mathrm{mL})$, PE anti-GPVI $\mathrm{mAb}(5 \mu \mathrm{g} / \mathrm{mL})$ or FITC anti-GPIX $\mathrm{mAb}(2.5 \mu \mathrm{g} / \mathrm{mL})$, respectively; AF647-annexin A5 $(5 \mu \mathrm{g} / \mathrm{mL})$ was added to measure PS exposure. Binding of VWF was analyzed after incubation with $2.5 \mu \mathrm{g} / \mathrm{mL}$ VWF and 0.5 $\mathrm{mg} / \mathrm{mL}$ ristocetin, followed by labeling with FITC anti-VWF Ab $(25 \mu \mathrm{g} / \mathrm{mL})$. Binding of coagulation factors was determined using OG488-labeled prothrombin or factor Xa $(5$ $\mu \mathrm{g} / \mathrm{mL}$ ). ${ }^{21}$ Platelet surface properties were analyzed with an Accuri 66 flow cytometer and software..$^{22}$

\section{Whole blood thrombus formation with platelet activation}

Washed coverslips were coated with microspots of collagen type I $(2 \mu \mathrm{L}, 50 \mu \mathrm{g} /$ $\mathrm{mL}$ ) and blocked with Hepes buffer $\mathrm{pH} 7.45$ containing 1\% BSA. Coated coverslips were mounted in a transparent parallel plate flow chamber ( $50 \mu \mathrm{m}$ depth, $3 \mathrm{~mm}$ width, $20 \mathrm{~mm}$ length). ${ }^{23}$ Thrombi with activated platelets were formed on collagen by a 4-min whole blood perfusion $\left(1000 \mathrm{~s}^{-1}\right)$, essentially as described elsewhere, ${ }^{24}$ but with modifications. 
In brief, citrated blood was mixed during flow by 1:10 (vol./vol.) co-perfusion with recalcification buffer ( $64 \mathrm{mM} \mathrm{CaCl}_{2}, 32 \mathrm{mM} \mathrm{MgCl}$ in Hepes buffer $\mathrm{pH}$ 7.45), prior to reaching the flow chamber. Blood perfusion was halted after thrombus contraction, but before visual appearance of fibrin fibers. Thrombi were then rinsed for 5 min with Hepes buffer $\mathrm{pH} 7.45$ containing $25 \mathrm{nM}$ thrombin and stained for GPIba (FITC mAb, $2 \mu \mathrm{g} / \mathrm{mL}$ ), GPVI (PE $\mathrm{mAb}, 1 \mu \mathrm{g} / \mathrm{mL}$ ), and/or PS exposure (AF647 annexin A5, $5 \mu \mathrm{g} / \mathrm{mL}$ ). Where indicated, the platelet thrombi were post-incubated with ADAM inhibitor GW280264X $(5 \mu \mathrm{M})^{25,26}$ or vehicle (DMSO) in Hepes buffer $\mathrm{pH} 7.45$ at $37^{\circ} \mathrm{C}$.

In an alternative protocol, to exclude thrombin generation, citrated blood was recalcified in the presence of $40 \mathrm{\mu M}$ PPACK ${ }^{4}$ and perfused over collagen as above, after which the thrombi were rinsed for 5 min with Hepes buffer $\mathrm{pH} 7.45$ containing $15 \mu \mathrm{M}$ SFLLRN. Subsequently, thrombi with SFLLRN-stimulated platelets were stained for binding of VWF (FITC mAb, $25 \mu \mathrm{g} / \mathrm{mL}$ ) or prothrombin $(10 \mu \mathrm{g} / \mathrm{mL})$ and PS exposure (AF647-annexin A5, $5 \mu \mathrm{g} / \mathrm{mL}$ ).

\section{Fibrin formation under flow conditions}

To measure the platelet fibrin-forming potential, ${ }^{27}$ citrated whole blood containing tirofiban $(5 \mu \mathrm{g} / \mathrm{mL})$ was perfused over collagen for $5 \mathrm{~min}\left(1000 \mathrm{~s}^{-1}\right)$, resulting in a single layer of adhered platelets. Platelets were post-perfused with $10 \mu \mathrm{M}$ ionomycin and 5 $\mathrm{mM} \mathrm{CaCl}_{2}$ in Hepes buffer $\mathrm{pH} 7.45$ for 5 min in the presence or absence of GW280264X. Fibrin formation was continuously monitored by fluorescence microscopy during flow of citrated plasma containing AF647-fibrinogen $(15 \mu \mathrm{g} / \mathrm{mL})$, co-perfused 1:10 with recalcification buffer, at a final shear rate of $250 \mathrm{~s}^{-1}$.

\section{Fluorescence microscopy}

Differential interference contrast (DIC) and multi-color fluorescence images were captured with a fast line-scanning Zeiss LSM7 system equipped with a $63 x$ oil-immersion objective (Carl Zeiss, Oberkochen, Germany). Fluorescence was recorded at excitation (emission) wavelengths of 488 (505-610), 532 (540-625) and 635 (>655) nm. Where indicated, brightfield and non-confocal fluorescent images (8 bit, 1360x1024 pixels, $142 \times 107 \mu \mathrm{m}$ ) were captured using an EVOS fluorescent microscope (Life Technologies, Bleiswijk, The Netherlands) equipped with an Olympus APLAN 60x oil objective. ${ }^{4}$ Image analysis was performed using Fiji image analysis software. ${ }^{28}$

\section{Statistical analysis}

Data are expressed as means \pm SD and analyzed with a paired samples t-test. P-values $<0.05$ were considered significant. Statistical analyses were performed using the SPSS Statistics 23 package (IBM, Armonk NY, USA). 


\section{Results}

\section{Strong platelet stimulation required for shedding of GPIba and GPVI}

To systematically study the roles of various signaling pathways implicated in GPIba and GPVI receptor shedding, washed human platelets were stimulated with a variety of agonists at near maximal concentrations, and then quantified for staining for the extracellular domains of GPIba and GPVI using flow cytometry. To check for possible internalization of the GPIb-V-IX complex, staining for GPIX was used as a control, as this glycoprotein chain is considered not to be cleaved by ADAM10/17. ${ }^{29}$ Whilst platelet stimulation with stable ADP (2MeS-ADP) did not affect the staining for GPIb $\alpha$, stimulation with CRP-XL (GPVI receptor agonist) or thrombin (PAR1/4 agonist) led to a modest reduction in staining (Figure 1A). No reduced staining for GPVI was observed under these conditions (Figure 1B). For all platelets, co-stimulation with CRP-XL plus thrombin led to a substantial, $60-80 \%$ reduction in both GPIba and GPVI staining; and the reduction was even more pronounced upon stimulation with the $\mathrm{Ca}^{2+}$-ionophore ionomycin (Figure $1 \mathrm{~A}$, $B)$. With none of these stimuli, a significantly reduced staining for GPIX was observed (Figure 1C), excluding the interference of internalization of the GPIb-V-IX complex. Of relevance, pretreatment of platelets with the ADAM10/17 inhibitor, GW280264X, ${ }^{25,30}$ essentially abrogated the loss of GPIb $\alpha$ and GPVI staining in platelets stimulated with CRP-XL/thrombin or ionomycin (Figure 1D-F), thus confirming the involvement of ADAM extracellular proteases.

Heterogeneity between platelets: association of GPIba and GPVI shedding with PS exposure

Using flow cytometry, we then studied the kinetics of decreases in GP surface levels (inhibited by GW280264X), as a measure of receptor shedding, in platelets stimulated with ionomycin. For all platelets, $90 \%$ of detectable GPIba was lost after 30 min stimulation, while $90 \%$ of detectable GPVI had already disappeared after $15 \mathrm{~min}$ (Figure 2A,B). This kinetic difference may be related to the higher copy number of GPIba in comparison to GPVI expressed per platelet, i.e. estimated as 18,800 and 9,600 copies, respectively. ${ }^{31}$ Stimulation of platelets with ionomycin is known to result in phospholipid scrambling and surface exposure of PS. ${ }^{32}$ Considering that membrane destabilization may trigger ADAM activity, we performed dual staining of platelets for PS exposure (AF-647 annexin A5) and either GPIba (FITC MAb) or GPVI (PE mAb) expression. Flow-cytometric dot plots indicated that the PS-exposing platelets indeed were low in GPIba and GPVI staining (Figure $2 \mathrm{C}$ ), whilst quantification showed that the staining for especially GPIba continued to decrease after full PS exposure (Figure 2D). Again, pretreatment of platelets with the ADAM10/17 inhibitor GW280264X blocked the GPIba and GPVI expression changes (Figure 2A, B).

A similar analysis was performed of platelets stimulated with CRP-XL plus thrombin. In comparison to ionomycin, CRP-XL/thrombin caused a slower loss of surface-expressed GPIb $\alpha$ and GPVI that continued for up to $3 \mathrm{~h}$ and longer. This loss again was antagonized by GW280264X (Figure 3A). For the platelet populations distinguished by dual labeling, 

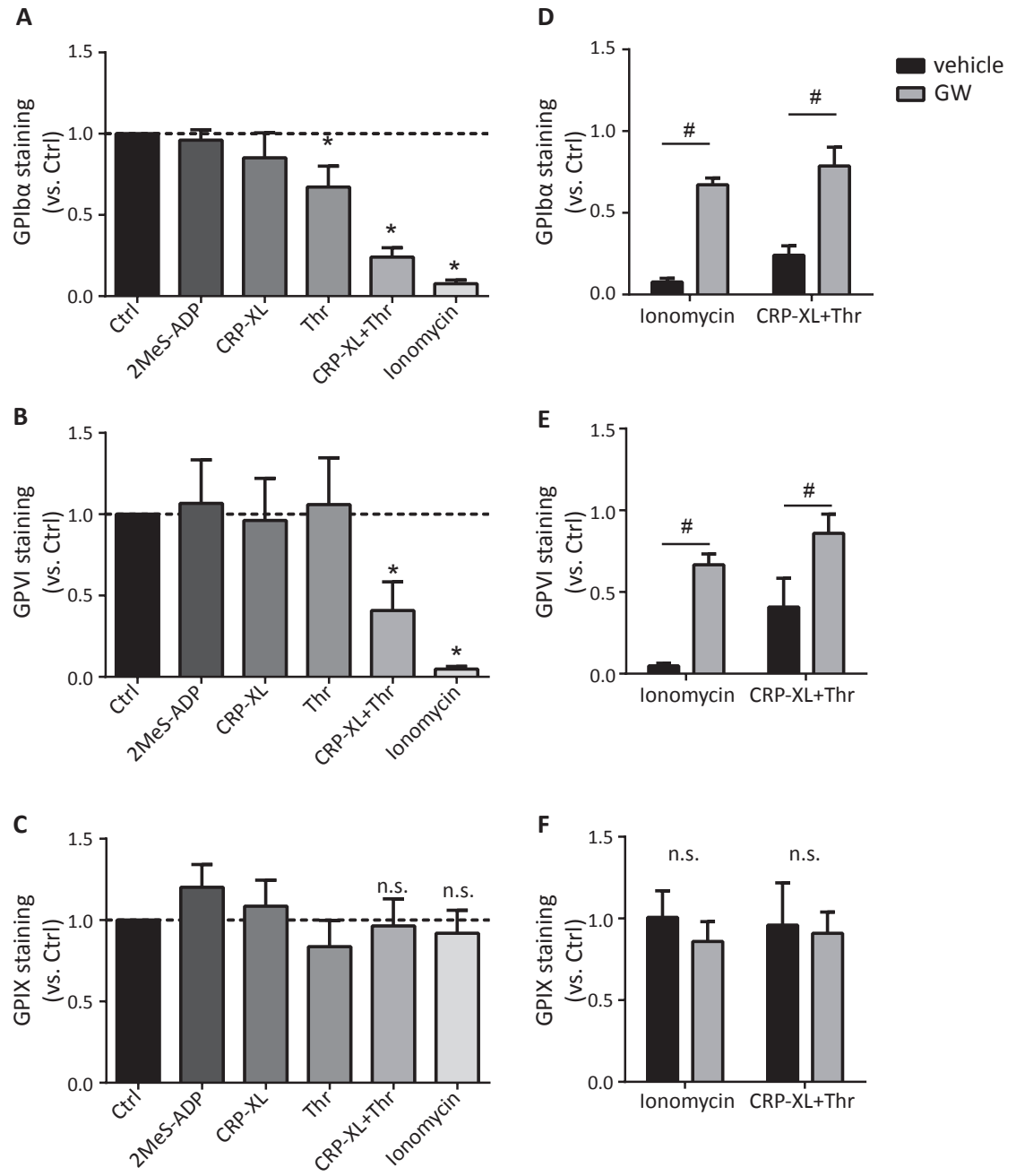

Figure 1: ADAM10/17 mediated glycoprotein shedding upon platelet stimulation with strong agonists. Washed platelets, 15 min pre-incubated with DMSO (vehicle) or ADAM17/10 specific inhibitor GW280264X (GW, $5 \mu \mathrm{M})$, were stimulated with 2MeS-ADP, CRP-XL, thrombin (Thr) or CRP-XL/Thr (GPIba, $\mathrm{n}=8)$ for 180 $\mathrm{min}$, or with ionomycin/ $\mathrm{CaCl}_{2}(\mathrm{GPIb \alpha}, \mathrm{n}=8)$ for $60 \mathrm{~min}$ (all $37^{\circ} \mathrm{C}$ ). Unstimulated platelets were used as control (Ctrl). Staining for GPIba (A, D), GPVI (B, E) and GPIX (C, F) was measured with optimized concentrations of FITC anti-GPIba mAb, PE anti-GPVI mAb or FITC anti-GPIX mAb using flow cytometry (mean fluorescence intensities). A-C, Effect of agonist stimulation on glycoprotein expression levels. Data were normalized to values of unstimulated platelets. D-F, Effect of GW treatment on glycoprotein expression level. Means \pm SD, $\mathrm{n}=3-5$ (8) ( $\mathrm{n} \geq 3$ donors), ${ }^{*} p<0.05$ vs. unstimulated platelets, ${ }^{\#} p<0.05$ vs. vehicle.

we observed that platelets with PS exposure appeared before the lowering of GPIba or GPVI staining (Figure 3B, C). Quantification of platelets with(out) PS exposure and shed/unshed receptors indicated that, at an intermediate time of $30 \mathrm{~min}$, a considerable fraction of platelets (16-44\%) presented with PS exposure with still high staining for 


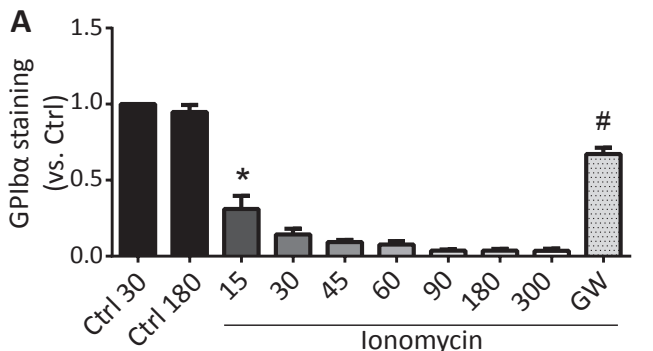

C
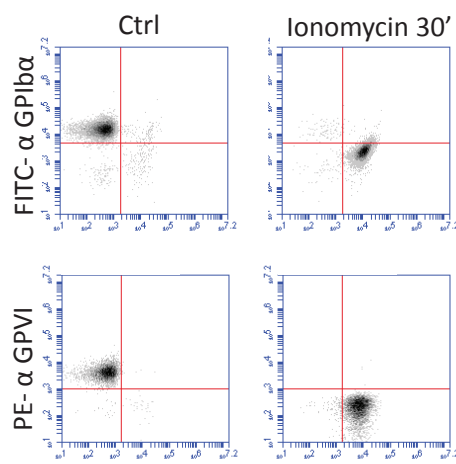

AF647-annexin A5
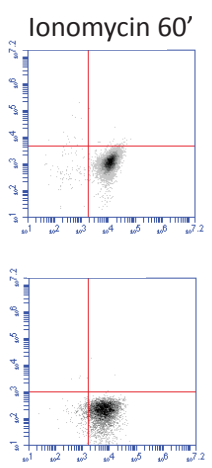

A5 AF647-annexin A5

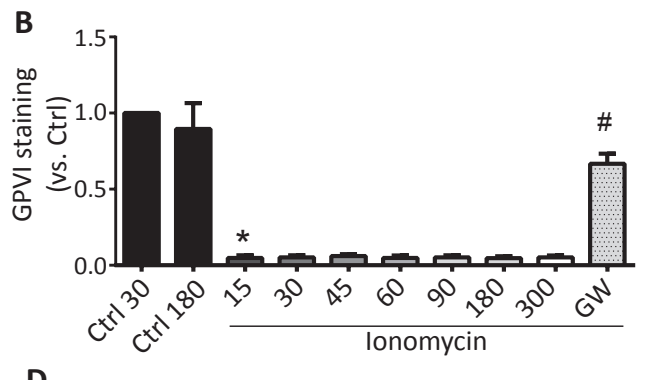

D
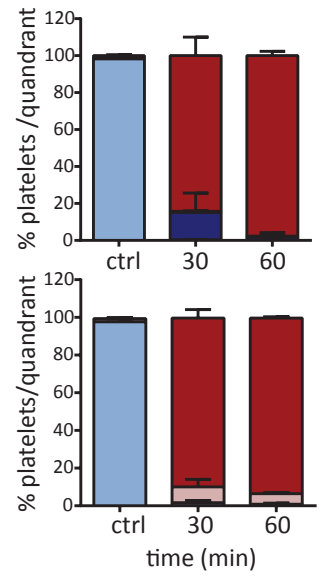

$\square$ GPIba low/PS+

$\square$ GPIba low/PS-

GPIba high/PS+

$\square$ GPIba high/PS-

$\square$ GPVI low/PS+

$\square$ GPVI low/PS-

GPVI high/PS+

$\square$ GPVI high/PS-

Figure 2: Comparable kinetics of glycoprotein receptor shedding and PS exposure in ionomycin stimulated platelets. Washed platelets, pre-incubated with vehicle or ADAM17/10 specific inhibitor GW280264X (GW, $5 \mu \mathrm{M})$, were left unstimulated (Ctrl) or were stimulated for indicated times (15-300 min) with ionomycin/ $\mathrm{CaCl}_{2}\left(37^{\circ} \mathrm{C}\right)$, and evaluated for glycoprotein expression by flow cytometry. A-B, ADAM-dependent decrease in surface expression of GPIba and GPVI over time. Mean fluorescence intensities were normalized to values of unstimulated platelets. C-D, Analysis of platelet populations after dual staining for GPIba (FITC mAb) or GPVI (PE mAb) and for PS exposure (AF647-annexin A5). Flow-cytometric events were separated into four platelet populations: i) $\mathrm{GPIb} \alpha / \mathrm{VI}^{\text {high }} \mathrm{PS}$, ii) $\mathrm{GPIb} \alpha / \mathrm{VI}^{\text {high }} \mathrm{PS}^{+}$, iii) $\mathrm{GPIb} \alpha / \mathrm{VI}^{\text {low }} \mathrm{PS}^{-}$and $\left.i v\right) \mathrm{GPIb} \alpha / \mathrm{VI}^{\text {low }} \mathrm{PS}^{+}$. Shown are representative dot plots and quantification of four quadrants. Means $\pm S D, n=3-5$ ( $n \geq 3$ donors); ${ }^{*} p<0.05$ vs. control, ${ }^{\sharp} p<0.05$ vs. vehicle.

GPIb $\alpha$ and GPVI (Figure 3D). These results may suggest that platelet PS exposure is a prerequisite for ADAM-mediated receptor shedding, similarly as has been reported for lymphocytes. ${ }^{18}$

Various other platelet agonists are known to cause glycoprotein shedding independently of receptor stimulation. These include the pro-apoptotic $\mathrm{BH} 3$ mimetic ABT-737, ${ }^{13}$ also inducing PS exposure; ${ }^{22}$ the PKC-activating agent PMA; $;, 33$ and the mitochondrial uncoupling agent, CCCP. ${ }^{7}$ In our hands, treatment of platelets with ABT737 resulted in a gradual (GW280264X-inhibitable) loss of GPIb $\alpha$ and GPVI expression, needing $>60$ min for completion (Figure S1A, B). Dual labeling with anti-GP mAb and AF647-annexin A5 indicated that the GPIba shedding at least in part preceded PS exposure, whereas the GPVI shedding was markedly slower than PS exposure (Figure S2A, B). However, ultimately the same population of PS-exposing platelets had lost their 
A

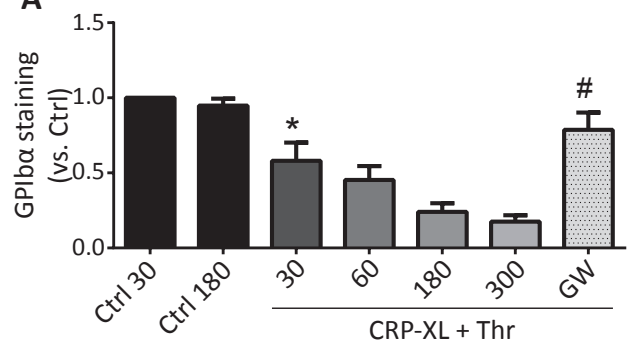

C
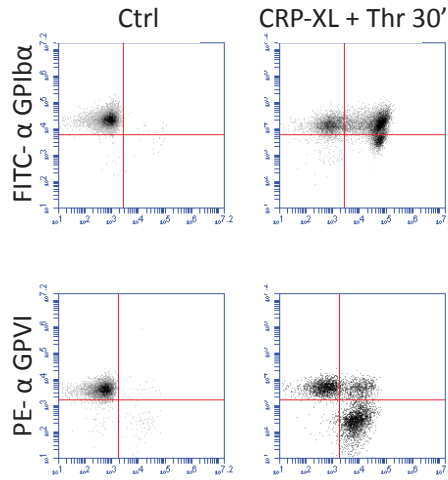

AF647-annexin A5

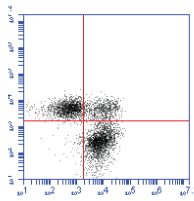

AF647-annexin A5

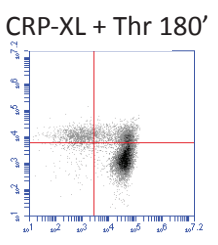

B

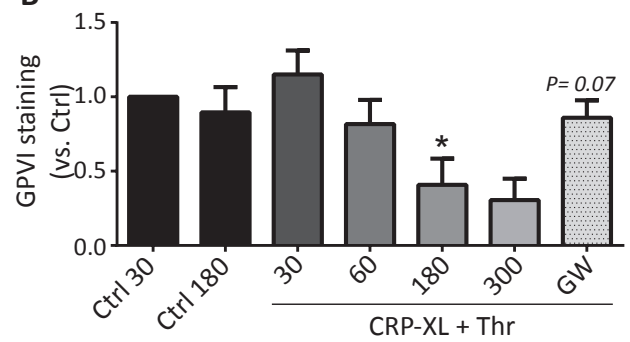

D

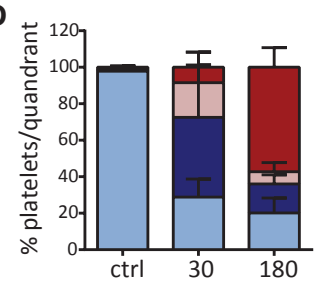

GPIba low/PS+

$\square$ GPIba low/PS-

- GPIba high/PS+

$\square$ GPIba high/PS-

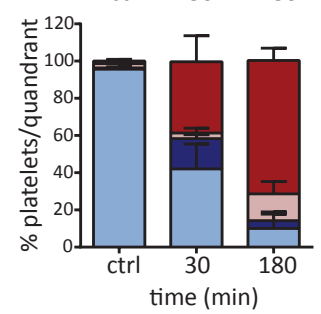

$\square$ GPVI low/PS+

$\square$ GPVI low/PS-

GPVI high/PS+

$\square$ GPVI high/PS-

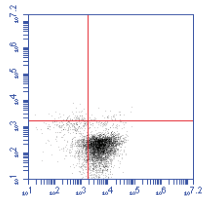

AF647-annexin A5

Figure 3: Glycoprotein receptor shedding following PS exposure in CXP-XL/thrombin stimulated platelets. Washed platelets, pre-incubated with vehicle or ADAM17/10 specific inhibitor GW280264X (GW, $5 \mu M$ ), were left unstimulated (Ctrl) or were stimulated with CRP-XL plus thrombin (Thr) for indicated times, and then evaluated for glycoprotein expression by flow cytometry. A-B, ADAM-dependent decrease in surface expression of GPIba and GPVI over time. C-D, Population analysis of platelets after dual staining for GPIba (FITC mAb) or GPVI (PE mAb) and for PS exposure (AF647-annexin A5). Four platelet populations are defined as in Figure 2. Note the gradual increase of the PS-exposing platelet populations, and the slower increase of populations with shed GPIb $\alpha$ or GPVI. Means \pm SD, $n=3-7$ ( $n \geq 3$ donors); ${ }^{*} p<0.05$ vs. control, ${ }^{*} p<0.05$ vs. vehicle.

glycoproteins. Stimulation with PMA resulted, for all platelets, in only limited GPIba shedding being still incomplete after $5 \mathrm{~h}$, whereas GPVI was not shed at all (Figure S1C, D). Double labeling indicated here that those platelets that did shed GPIba were PS negative (Figure S2C, D). Platelet treatment with CCCP caused even slower shedding, especially of GPVI, requiring up to $5 \mathrm{~h}$ for becoming measurable (Figure S1E, F). With CCCP, also limited PS exposure was measured (see below). Together, these data indicated that agonist-induced glycoprotein receptor shedding and PS exposure occur in the same platelet populations, although these processes in kinetics seem to be independent events.

To investigate this in more detail, we studied the platelets from a Scott syndrome patient, which respond normally in aggregation, but are deficient in PS exposure when stimulated with $\mathrm{Ca}^{2+}$-mobilizing agonists, such as ionomycin or CRP-XL/thrombin. ${ }^{22,34}$ Strikingly, the Scott syndrome platelets, regardless of the stimulus applied - ionomycin, 

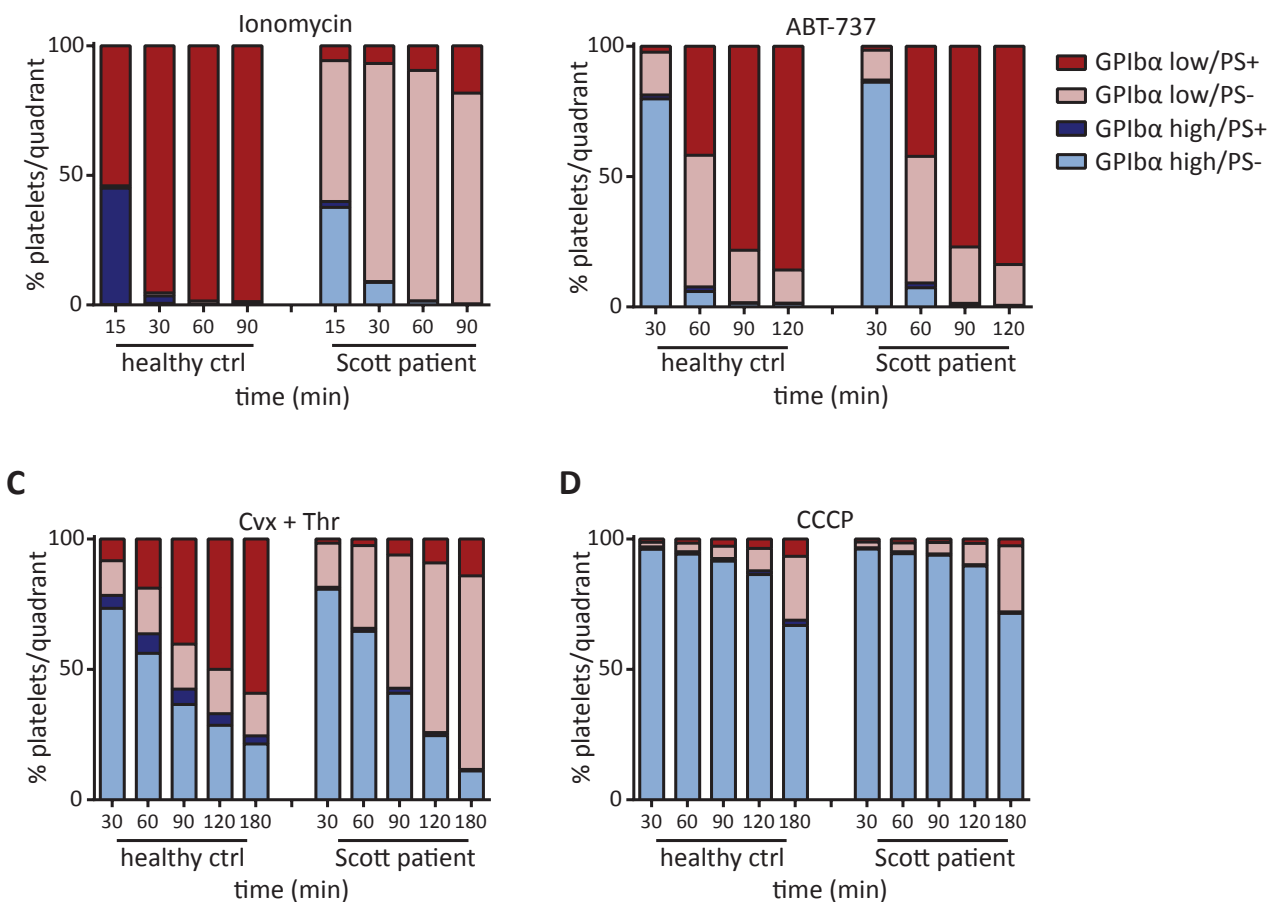

Figure 4: Normal glycoprotein shedding in Scott syndrome platelets with defective PS exposure. Platelets from a healthy control subject or a Scott patient were stimulated with $10 \mu \mathrm{M}$ ionomycin (A), $10 \mu \mathrm{M}$ ABT737 (B), $100 \mathrm{ng} / \mathrm{mL}$ convulxin and $4 \mathrm{nM}$ thrombin (C), or $100 \mu \mathrm{M} \mathrm{CCCP} \mathrm{(D)} \mathrm{at} 37^{\circ} \mathrm{C}$. GPIba expression and PS exposure were measured at indicated time points. Platelets were separated into four populations and

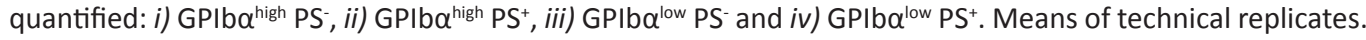

ABT-737, convulxin/thrombin or CCCP -, displayed high GPIba shedding to the same extent as the platelets from a control donor (Figure 4A-D). Earlier, we have verified that these Scott syndrome platelets show normal agonist-induced $\mathrm{Ca}^{2+}$ fluxes. ${ }^{35}$ The current data hence indicate that the glycoprotein shedding is regulated via a mechanism that is independent of phospholipid membrane scrambling and PS exposure.

\section{Pathways of glycoprotein shedding: key roles of calcium rise and caspase activity}

In order to unravel the signaling pathways accountable for receptor shedding in response to the various agents (ionomycin, CRP-XL/thrombin, ABT-737, PMA and CCCP), we applied a panel of inhibitors blocking specific signaling events. Therefore, the platelets were pretreated with: ADAM10/17 inhibitor GW280264X, ${ }^{25}$ calpain inhibitor MDL-28170, ${ }^{5}$ pan-caspase inhibitor Q-VD-OPh, ${ }^{22}$ general PKC inhibitor Ro-318425, ${ }^{36}$ cytosolic $\mathrm{Ca}^{2+}$ chelator $\mathrm{dm}$-BAPTA AM, ${ }^{22}$ or the $\mathrm{Ca}^{2+}$ entry inhibitor $2 \mathrm{APB} \cdot{ }^{37}$ The cells were then activated with a selected agent. The combined effects of agonist and inhibitors (see Table S1) were converted into a heat map, representing both the relative strength of agonists to induce GPIba shedding (shown in red) and the efficacy of inhibitors to 


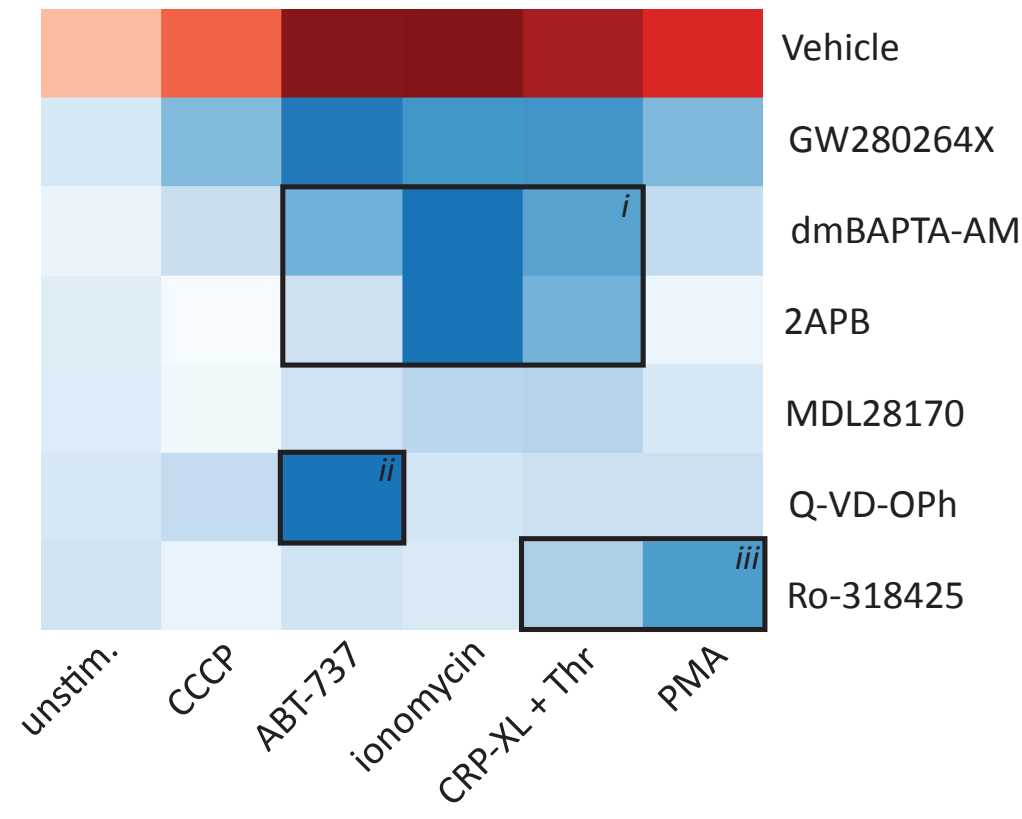

Figure 5: Analysis of signaling pathways implicated in agonist-induced glycoprotein shedding. Washed platelets were pre-treated with the ADAM10/17 inhibitor GW280264X, the calpain inhibitor MDL-28170, the pan-caspase inhibitor Q-VD-OPh, the general PKC inhibitor Ro-318425, the $\mathrm{Ca}^{2+}$ chelator dm-BAPTA AM or the $\mathrm{Ca}^{2+}$ entry inhibitor 2APB. Samples were then stimulated with ionomycin/ $\mathrm{CaCl}, \mathrm{CRP}-\mathrm{XL}$ plus thrombin, PMA, ABT-737 or CCCP, as indicated. Data were normalized to the values of unstimulated platelets without any inhibitors. Shown is a heat map of effect of agonists on GPIba shedding (red), and of the relative antagonizing effects of inhibitors (blue). Note the three clusters (top-down), representing a role of (i) $\mathrm{Ca}^{2+}$ elevation, (ii) caspase and (iii) PKC activity. See further Table S1.

antagonize agonist effects (shown in blue) (Figure 5). This heatmap identified as main signaling pathways for GPIb $\alpha$ shedding: (i) high $\mathrm{Ca}^{2+}$ elevation (inhibited by dm-BAPTA $\mathrm{AM}, 2 \mathrm{APB}$ or absence of extracellular $\mathrm{CaCl}_{2}$ ) with ionomycin $>\mathrm{CRP}-\mathrm{XL} /$ thrombin $>\mathrm{ABT}$ 737; (ii) caspase activity (inhibited by Q-VD-OPh) with ABT-737; and (iii) PKC activation (inhibited by Ro-318425) with PMA > CRP-XL/thrombin. A minor involvement of calpain was seen in ionomycin-treated platelets.

\section{Heterogeneous shedding of GPIba and GPVI in thrombi}

Since GPIb $\alpha$ and GPVI are key receptors in the regulation of collagen-dependent thrombus formation under flow, ${ }^{1,4}$ we reasoned that the ADAM-mediated cleavage of either or both receptors may serve to secondarily abrogate the adhesion of platelets that are aggregated into thrombi. To investigate this, thrombi were allowed to form on a collagen surface, under conditions were thrombin was generated as well. In this setting, the thrombi are known to consist of two populations of platelets, i.e. pseudopodcontaining platelets in aggregates, surrounded by patches of balloon-shaped, PS-exposing platelets around the aggregates. ${ }^{1,34}$ Concerning the aggregated platelets, no difference in staining for GPIba or GPVI expression was seen after up to $3 \mathrm{~h}$ incubation (Figure 6A, B). However, the balloon-shaped, PS-exposing platelets did no longer stain for GPIb $\alpha$ or GPVI. 
Incubation of thrombi in the presence of GW280264X retained the staining for GPIb $\alpha$ and GPVI of the balloon-shaped, PS-exposing platelets. In this case, the presence of PS-exposing platelets was not affected by the GW280264X treatment $(9.4 \pm 3.0 \%$ SAC vs. $12.1 \pm 6.8 \% \mathrm{SAC}$ for controls, means $\pm \mathrm{SD}, \mathrm{n}=3, \mathrm{p}=0.39$ ). In subsequent studies, we post-activated the thrombi formed on collagen with ionomycin in order to increase the number of ballooned, PS-exposing platelets. This resulted in a marked, overall reduction in staining for GPIba and GPVI (Figure S3). Again, this reduction was antagonized by

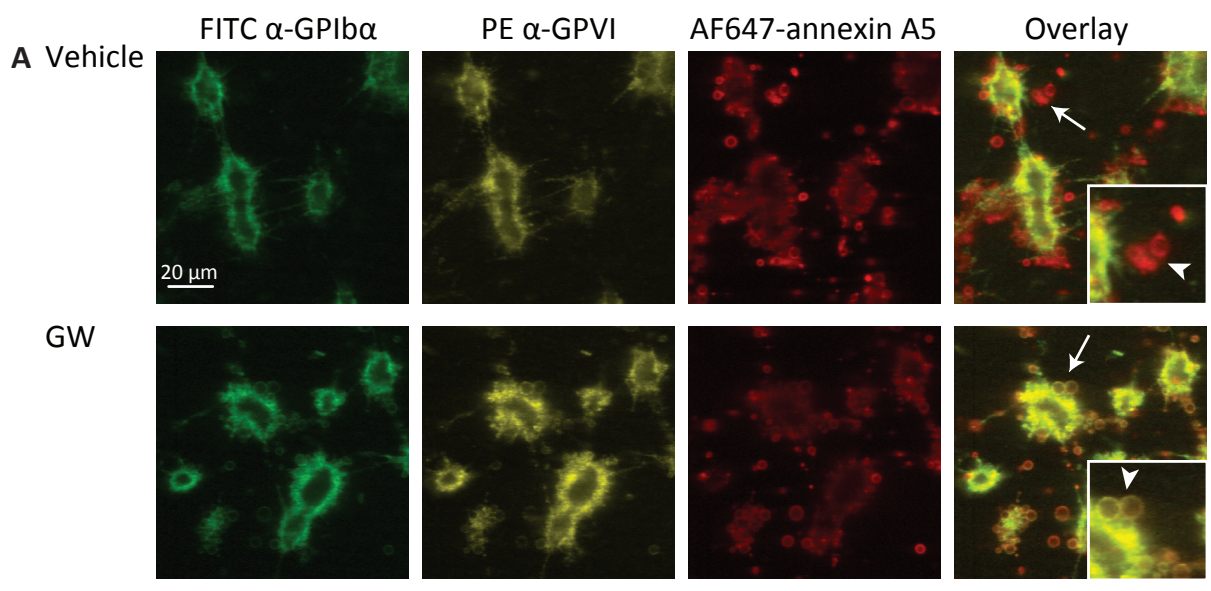

B

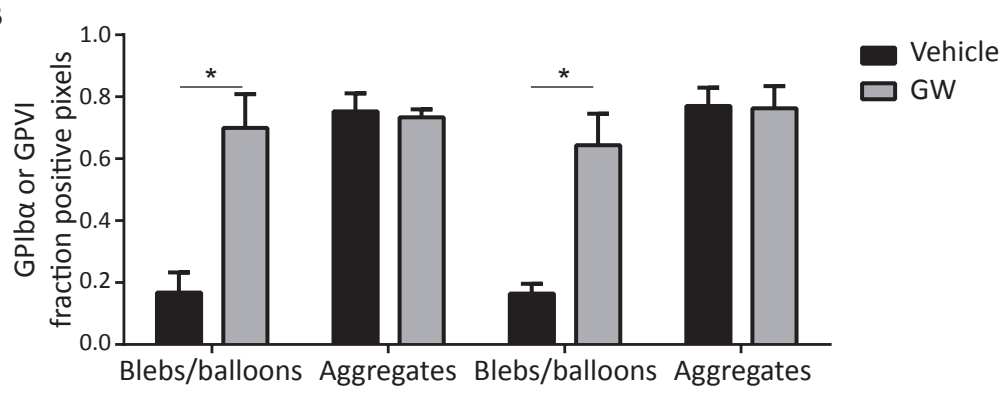

GPIba

GPVI

Figure 6: Selective loss of GPIba and GPVI in high- $\mathrm{Ca}^{2+}$, PS-exposing platelets in thrombi. Platelet thrombi were formed in flow chambers on collagen type I using whole blood perfusion at a wall shear rate of 1000 $\mathrm{s}^{-1}$. The thrombi were post-activated with thrombin, and incubated with vehicle or GW280264X (GW) for up to 180 min at $37^{\circ} \mathrm{C}$; staining then was for GPIb $\alpha$ and GPVI expression and PS exposure. A, Representative confocal fluorescence images of GPIb $\alpha$ and GPVI expression and PS exposure; bar $=20 \mu \mathrm{m}$. PS-exposing, ballooned platelets are indicated by white arrows and arrowheads (also $2 x$ magnified inserts). B, Regionsof-interest corresponding to aggregated platelets and ballooned platelets were analyzed for fluorescence staining. Pixels representing GPIb $\alpha$ - or GPVI-positive platelets were determined, and expressed as percentages of surface area coverage of ballooned or aggregated platelets. Means $\pm S D, n=3, * p<0.05$. 
GW280264X. From these data, we deduced that only the high-Ca ${ }^{2+}$, PS-exposing platelets in thrombi had lost their GPIba and GPVI receptors, underscoring that the ADAMdependent glycoprotein shedding is preferably induced in platelets with high $\mathrm{Ca}^{2+}$ levels.

\section{Functional consequences of shedding of GPIba and GPVI}

Subsequently, we studied the functional consequences of glycoprotein receptor shedding on activated platelets. We reasoned that cleavage of the whole extracellular domain of these abundantly expressed glycoproteins may reduce the platelet adhesive properties, whilst increasing the capacity to bind coagulant factors. To assess this, we stimulated washed platelets with ionomycin, ABT-737 or CRP-XL/SFLLRN (replacing thrombin, to prevent fibrin formation) to cause GPIba and GPVI shedding, and then measured their capability to bind VWF in the presence of ristocetin by flow cytometry. All agonists markedly reduced VWF binding, such in accordance with the overall lower level of extracellular GPIba (Figure 7A i). Preincubation of platelets with GW280264X or $5 \mathrm{G} 6 \mathrm{Fab}$ fragment, which blocks the ADAM17 cleavage site in GPIb $\alpha,{ }^{10}$ almost completely restored the ability of VWF binding (Figure 7A $i$ ).

We also determined the consequences of glycoprotein shedding for binding of the coagulation factors, OG488-labeled prothrombin and factor Xa. Similarly as reported before$^{21}$ in platelets stimulated with ionomycin, ABT-737 or CRP-XL/SFLLRN, both Gla-domain containing coagulation factors showed high binding only to PS-exposing platelets (Figure 7Aii-iii). Markedly, inhibition of ADAM activity with GW280264X caused a decreased labeling of both OG488-prothrombin and OG488-factor Xa for all platelets (Figure S4B), and for the population of PS-exposing platelets (Figure 7A ii-iii), regardless of the agonist used. Note that in the latter case PS-exposing platelets were identified with AF647-annexin A5, which was added 10 min after the labeled coagulation factors, i.e. a protocol in which annexin $\mathrm{A} 5$ did not interfere with coagulation factor labeling. As the 5 G6 Fab fragment had a similar effect as treatment with GW280264X (Figure 7A ii-iii), we concluded that cleavage of GPIba is a main determinant of the regulation of coagulation factor binding.

Similarly, the thrombi with PS-exposing platelets formed on collagen were stained for prothrombin binding. As reported before, ${ }^{21}$ OG488-prothrombin fluorescence was found to co-localize with PS exposure (Figure 7B). We furthermore observed that ADAM inhibition with GW280264X suppressed prothrombin binding on (high-Ca ${ }^{2+}$ ) PS-exposing platelets (Figure 7B), which is in line with the flow cytometry results. Annexin A5 staining was not affected by GW280264X. Staining for VWF indicated low staining levels on PSexposing platelets (not shown), again in agreement with the requirement of non-cleaved GPIba for VWF binding.

To further assess the functional consequences of the altered coagulation factor binding properties of PS-exposing platelets with shed receptors, we determined their role in the formation of fibrin clots. Therefore, we generated a layer of adhered platelets on collagen by flow perfusion of integrin-inhibited blood, and superfused this secondarily with recalcified plasma to trigger thrombin and fibrin generation. In this setup, prior treatment of the adhered platelets with $\mathrm{Ca}^{2+}$-elevating ionomycin resulted in accelerated 

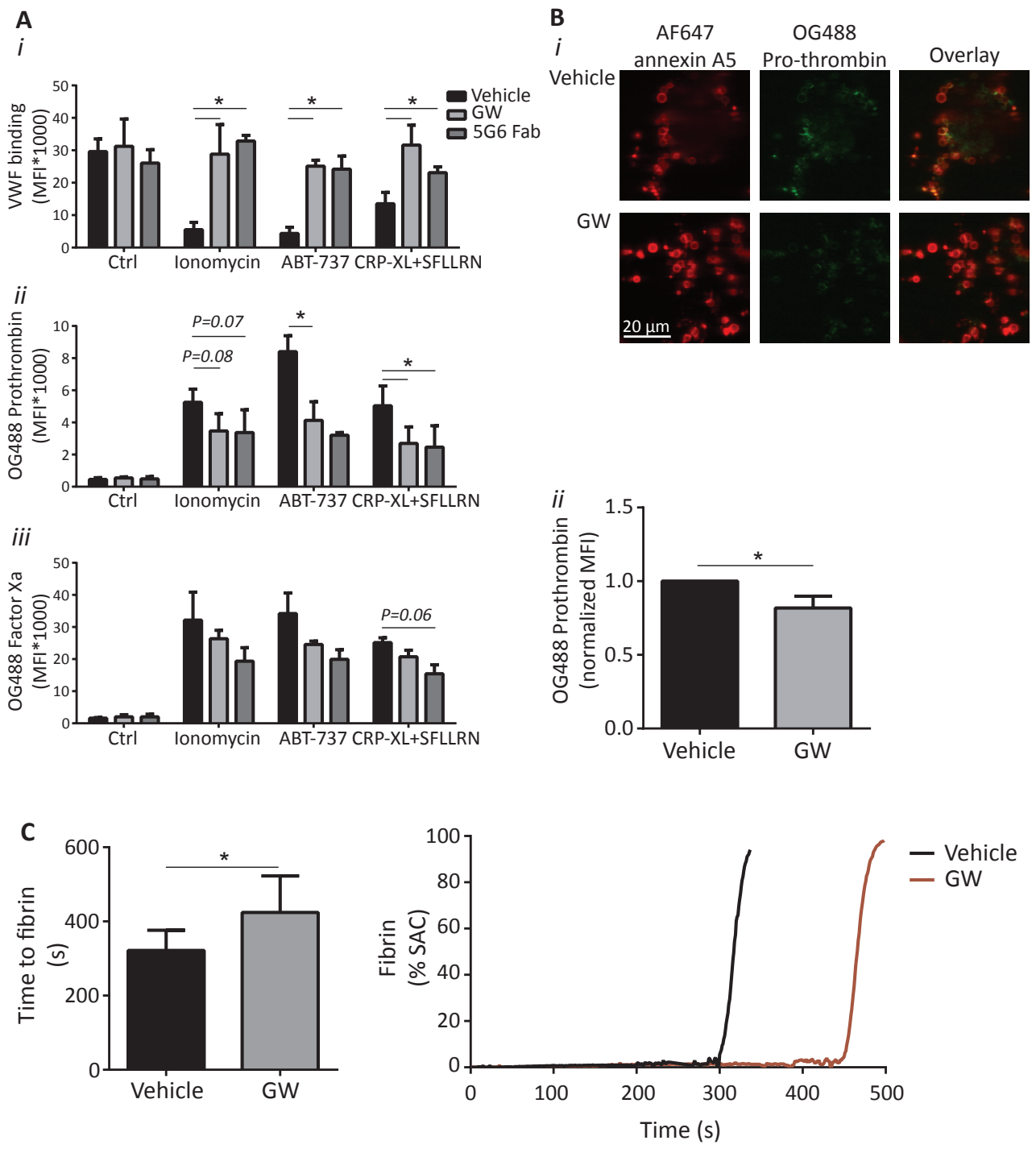

Figure 7: Glycoprotein receptor shedding enforces a platelet procoagulant phenotype. A, Washed platelets were pre-incubated with vehicle, GW280264X (GW, $5 \mu \mathrm{M}, 15 \mathrm{~min}$ ) or $5 \mathrm{G} 6$ Fab fragment, known to block ADAM17-mediated shedding of GPIba (10 $\mu \mathrm{g} / \mathrm{mL}, 15 \mathrm{~min})$. After $60 \mathrm{~min}$ of stimulation $\left(37^{\circ} \mathrm{C}\right)$ with ionomycin, ABT-737 or CRP-XL plus SFLLRN, flow cytometry was used to measure platelet binding of: (i) VWF with fluorescent anti-VWF mAb (plus ristocetin), (ii) OG488-prothrombin, or (iii) OG488-factor Xa. Prothrombin and factor $\mathrm{Xa}$ binding was also assessed for the PS-exposing platelet population, using AF647annexin A5. Means $\pm S D, n=3-4,{ }^{*} p<0.05$. B, Platelet thrombi were formed on collagen and post-activated with SFLLRN in the presence of vehicle or GW, followed by staining for OG488-prothrombin. Representative images (i) and quantification (ii) of prothrombin fluorescence. Means $\pm S D, n=3,{ }^{*} p<0.05$. C, Fibrin formation at a layer of adhered platelets on collagen, post-stimulated with ionomycin in the presence of vehicle or GW. Fibrin formation was induced by flow perfusion of recalcified normal plasma at $250 \mathrm{~s}^{-1}$. Shown are times to fibrin formation and representative time traces of fibrin formation (expressed as surface area coverage (\%SAC)). Means $\pm S D, n=6,{ }^{*} p<0.05$. 
fibrin formation from the platelet surface, an effect that was markedly delayed in the presence of GW280264X (Figure 7C). From these results, we concluded that $\mathrm{Ca}^{2+}$-induced glycoprotein shedding enhances the clotting potential of PS-exposing platelets.

\section{Discussion}

In the present paper, we provide first evidence for heterogeneity between activated platelets in ADAM10/17-mediated shedding of extracellular GPIba and GPVI, leading to populations of platelets with near complete glycoprotein shedding and populations without shedding. These pathways include: (i) a high $\mathrm{Ca}^{2+}$ rise, e.g. induced by collagen/thrombin receptor stimulation or ionomycin in platelets, also showing PS exposure and ballooning; (ii) PKC stimulation; (iii) caspase-mediated apoptosis, such as evoked by the BH3 mimetic ABT-737; in this case preceding PS exposure. Our data indicate that, in response to either $\mathrm{Ca}^{2+}$-mobilizing or pro-apoptotic stimuli, essentially the platelet population exhibiting ADAM-induced glycoprotein shedding greatly overlap with the population of PS-exposing platelets. Importantly, shedding of GPIba occurred normally in platelets from a Scott patient with defective PS exposure and unaffected other platelet responses. ${ }^{34,38}$ Accordingly, although PS exposure and shedding coincide, it appears that the major membrane disturbances caused by phospholipid scrambling and balloon formation are not a prerequisite for ADAM-mediated proteolysis. Rather, our data indicate that the platelets showing PS exposure and receptor shedding share a common denominator upstream in the regulatory pathways (schematically illustrated in Figure S5). The present data with platelets thus deviate from a recent report on lymphocytes, where PS exposure was considered to be necessary for ADAM17 activity. ${ }^{18}$

A new finding, from our flow cytometric data, is that ADAM-induced glycoprotein shedding appears as an all-or-nothing event. This is concluded from the observations that shedding of the two glycoproteins, GPIba (via ADAM17) and GPVI (via ADAM10): (i) mostly occur in the same platelet populations regardless of the agonist (with PMA as an exception, see below); (ii) in individual platelets proceeds to near completion, thus leading to a separation of populations of shed and non-shed platelets. Of note, the ADAM10/17 inhibitor GW280264X showed near complete inhibition, which was also the case with the ADAM inhibitor GI254023X ( $5 \mu \mathrm{M}$, about $80 \%$ ), while matrix metalloproteinase inhibitors were without effect (not shown). We speculate that the residual protein cleavage is due to another ADAM isoform, e.g. ADAM9, which is expressed at 600 copies per platelet. ${ }^{31}$

Investigations so far show that ADAM10 and ADAM17 cleave multiple glycoprotein substrates with at least partial redundancy. ${ }^{9}$ Yet, ADAM17 may preferentially cleave, next to GPIba, JAM-A, tetraspanin 9 and semaphorins; whereas ADAM10 can preferentially shed GPVI and CD84. ${ }^{33,39}$ The substrates for cleavage may also depend on the type of agonist, but this has not been well clarified. ${ }^{11}$ In terms of signaling pathways, other authors have proposed an inhibitory role of calmodulin, ${ }^{9}$ and a stimulating role of the p38 
MAPK pathway ${ }^{14}$ in ADAM-mediated glycoprotein shedding. The biochemical mechanism of protease activation of platelet ADAM isoforms is still unclear. ${ }^{40,41}$ Our data point to a positive, rather than a negative role, of elevated cytosolic $\mathrm{Ca}^{2+}$ in glycoprotein shedding.

We find that, in general, GPIb $\alpha$ and GPVI are cleaved to the same extent on individual platelets stimulated by agonists, with the exception of PMA which preferentially sheds GPIb $\alpha$. This may imply a specific PKC-dependent activation of ADAM17 with GPIb $\alpha$ as preferential substrate. This is in line with observations with murine embryonic fibroblasts, showing that PKC activation fails to activate ADAM10. ${ }^{42}$ The two major pathways causing glycoprotein shedding - $\mathrm{Ca}^{2+}$ elevation and caspase activity - appear to operate on the level of single platelets, i.e. most likely those populations of platelets with highest $\mathrm{Ca}^{2+}$ fluxes or most active caspase activities. Furthermore, our data also indicate redundancy between these pathways, since chelation of cytosolic $\mathrm{Ca}^{2+}$ in apoptotic platelets resulted in lower shedding. This is in line with earlier observations that ABT-737 can form a platelet population with elevated $\mathrm{Ca}^{2+}$ levels. ${ }^{22}$

In thrombi with aggregated and procoagulant platelets, we find high ADAMdependent glycoprotein shedding on ballooned/blebbed platelets that expose PS. Furthermore, we demonstrate for the first time that glycoprotein shedding enhances prothrombin binding and accelerates fibrin formation at the platelet surface. This corresponds to data from others that thrombi formed under flow conditions are high in ADAM10 activity. ${ }^{43}$ Platelet PS exposure is accompanied by less adhesion due to calpain-induced integrin cleavage, and such platelets are excluded from tight plateletplatelet contacts. ${ }^{5}$ The present data indicate that these platelets are also unable to bind VWF through GPIba. With the evidence that both the GPIb-V-IX complex (indirectly via VWF) and GPVI can bind fibrin, ${ }^{44-46}$ our results intriguingly indicate that the platelet population that contributes most to fibrin formation (by PS exposure and coagulation factor binding) does no longer express these glycoprotein receptors. This implies that a fibrin-dependent signaling via GPIb-V-IX and GPVI must be more important in platelets incorporated into aggregates than in procoagulant platelets. GPIba in addition provides a binding site for $\alpha$-thrombin. ${ }^{47}$ However, this binding does not seem to play a role in the generation of thrombin and fibrin at PS-exposing platelets, given the rapid shedding of GPIb $\alpha$ from such platelets. The latter is in agreement with published data that platelets treated with the GPIba-cleaving endopeptidase display normal thrombin generation, ${ }^{48}$ although others have suggested differently. ${ }^{49}$ Taken together, our data indicate that in thrombus formation - GPIba and GPVI shedding: (i) is primarily induced in platelet populations displaying high $\mathrm{Ca}^{2+}$ elevation, (ii) occurs independently of PS exposure, and (iii) enhances coagulant activity. 


\section{Key points}

- Populations of activated platelets shed GPIba and GPVI by ADAM activity via pathways associated with, but not secondary to PS exposure.

- In thrombi, ADAM-induced shedding is limited to PS-exposing platelets and thereby enhances procoagulant activity.

\section{Acknowledgements}

This work was supported by the Netherlands Landsteiner Foundation for Blood Transfusion Research (1006). P.E.J.M. is supported by the Cardiovascular Center Maastricht, F.S. by the Alexander von Humboldt foundation, M.M.P.C.D. by the Netherlands Heart Foundation (Dr. E. Dekker grant nr. 2012T079) and P.E.B. by the NIH (NIH RO1 Grant HL071544).

\section{Authorship}

Contribution: C.C.F.M.J.B., F.S., T.M., T.G.M. and M.A.H.F. performed experiments and analyzed data; P.E.B., M.M.P.C.D., P.W.C. and R.L. contributed essential research tools; P.E.J.M and J.W.M.H designed and coordinated the research; C.C.F.M.J.B., P.E.J.M. and J.W.M.H. wrote the paper. All authors have seen and approved the final manuscript.

Conflict of interest: The authors declare no relevant conflicts of interest exist.

Correspondence: Johan W. M. Heemskerk PhD, Dept. Biochemistry (CARIM), Maastricht University, PO Box 616, 6200 MD Maastricht, The Netherlands. Tel: +31-43-3881671, fax: +31-43-3884159; e-mail: jwm.heemskerk@maastrichtuniversity.nl 


\section{References}

1. Versteeg HH, Heemskerk JWM, Levi M, Reitsma PH. New fundamentals in hemostasis. Physiol Rev. 2013;93:327-58.

2. Berndt MC, Metharom P, Andrews RK. Primary haemostasis: newer insights. Haemophilia. 2014;20:1522.

3. Swieringa F, Kuijpers MJE, Heemskerk JWM, van der Meijden PEJ. Targeting platelet receptor function in thrombus formation: The risk of bleeding. Blood Rev. 2014;28:9-21.

4. De Witt SM, Swieringa F, Cavill R, Lamers MME, van Kruchten R, Mastenbroek T, et al. Identification of platelet function defects by multi-parameter assessment of thrombus formation. Nat Commun. 2014;5:4257. 5. Mattheij NJ, Gilio K, van Kruchten R, Jobe SM, Wieschhaus AJ, Chishti AH, et al. Dual mechanism of integrin $\mathrm{a}_{\| 1} \mathrm{~b}_{3}$ closure in procoagulant platelets. J Biol Chem. 2013;288(19):13325-36.

6. Solari FA, Mattheij NJ, Burkhart JM, Swieringa F, Collins PW, Cosemans JM, et al. Combined quantification of the global proteome, phosphoproteome, and proteolytic cleavage to characterize altered platelet functions in the human scott syndrome Mol Cell Proteomics. 2016;15(10):3154-69.

7. Bergmeier W, Piffath CL, Cheng G, Dole VS, Zhang Y, von Andrian UH, et al. Tumor necrosis factor-alphaconverting enzyme (ADAM17) mediates GP1balpha shedding from platelets in vitro and in vivo. Circ Res. 2004;95(7):677-83.

8. Gardiner EE, Arthur JF, Kahn ML, Berndt MC, Andrews RK. Regulation of platelet membrane levels of glycoprotein VI by a platelet-derived metalloproteinase. Blood. 2004;104(12):3611-7.

9. Gardiner EE, Karunakaran D, Shen Y, Arthur JF, Andrews RK, Berndt MC. Controlled shedding of platelet glycoprotein (GP)VI and GPIb-IX-V by ADAM family metalloproteinases. J Thromb Haemost. 2007;5(7):15307.

10. Liang X, Russell SR, Estelle S, Jones LH, Cho S, Kahn ML, et al. Specific inhibition of ectodomain shedding of glycoprotein lba by targeting its juxtamembrane shedding cleaving site. J Thromb Haemost. 2013;11(12):2155-62.

11. Bender M, Hofmann S, Stegner D, Chalaris A, Bösl M, Braun A, et al. Differentially regulated GPVI ectodomain shedding by multiple platelet-expressed proteinases. Blood. 2010;116(17):3347-55.

12. Wang Z, Shi Q, Yan R, Liu G, Zhang W, Dai K. The role of calpain in the regulation of ADAM17-dependent GPIbalpha ectodomain shedding. Arch Biochem Biophys. 2010;495(2):136-43.

13. Schoenwaelder SM, Jarman KE, Gardiner EE, Hua M, Qiao J, White MJ, et al. Bcl-xL-inhibitory BH3 mimetics can induce a transient thrombocytopathy that undermines the hemostatic function of platelets. Blood. 2011;118(6):1663-74.

14. Canault M, Duerschmied D, Brill A, Stefanini L, Schatzberg D, Cifuni SM, et al. p38 mitogen-activated protein kinase activation during platelet storage: consequences for platelet recovery and hemostatic function in vivo. Blood. 2010;115(9):1835-42.

15. Al-Tamimi M, Tan CW, Qiao J, Pennings GJ, Javadzadegan A, Yong AS, et al. Pathological shear triggers shedding of vascular receptors: a novel mechanism for down-regulation of platelet glycoprotein VI in stenosed coronary vessels. Blood. 2012;119(18):4311-20.

16. Wang Z, Cai F, Hu L, Lu Y. The role of mitochondrial permeability transition pore in regulating the shedding of the platelet GP1ba ectodomain. Platelets. 2014;25(5):373-81.

17. Zhang P, Du J, Zhao L, Wang Z, Zhang Y, Yan R, et al. The role of intraplatelet reactive oxygen species in the regulation of platelet glycoprotein Iba ectodomain shedding. Thromb Res. 2013;132(6):696-701.

18. Sommer A, Kordowski F, Büch J, Maretzky T, Evers A, Andrä J, et al. Phosphatidylserine exposure is required for ADAM17 sheddase function. Nat Commun. 2016;7:11523.

19. Castoldi E, Collins PW, Williamson PL, Bevers EM. Compound heterozygosity for 2 novel TMEM16F mutations in a patient with Scott syndrome. Blood. 2011;117(16):4399-400.

20. Gilio K, Munnix IC, Mangin P, Cosemans JMEM, Feijge MAH, van der Meijden PEJ, et al. Non-redundant roles of phosphoinositide 3-kinase isoforms $\mathrm{a}$ and $\mathrm{b}$ in glycoprotein VI-induced platelet signaling and thrombus formation. J Biol Chem. 2009;284:33750-62.

21. Berny MA, Munnix IC, Auger JM, Schols SE, Cosemans JM, Panizzi P, et al. Spatial distribution of factor Xa, thrombin, and fibrin(ogen) on thrombi at venous shear. PLos One. 2010;5(4):e10415.

22. van Kruchten R, Mattheij NJ, Saunders C, Feijge MAH, Wolfs JL, Collins PW, et al. Both TMEM16Fdependent and TMEM16F-independent pathways contribute to phosphatidylserine exposure in platelet 
apoptosis and platelet activation. Blood. 2013;121(10):1850-7.

23. van Kruchten R, Cosemans JMEM, Heemskerk JWM. Measurement of whole blood thrombus formation using parallel-plate flow chambers - a practical guide. Platelets. 2012;23:229-42.

24. Swieringa F, Baaten CC, Verdoold R, Mastenbroek TG, Rijnveld N, van der Laan KO, et al. Platelet control of fibrin distribution and microelasticity in thrombus formation under flow. Arterioscler Thromb Vasc Biol. 2016;36(4):692-9.

25. Donners MM, Wolfs IM, Olieslagers S, Mohammadi-Motahhari Z, Tchaikovski V, Heeneman S, et al. A disintegrin and metalloprotease 10 is a novel mediator of vascular endothelial growth factor-induced endothelial cell function in angiogenesis and is associated with atherosclerosis. Arterioscler Thromb Vasc Biol. 2010;30(11):2188-95.

26. Ludwig A, Hundhausen C, Lambert MH, Broadway N, Andrews RC, Bickett DM, et al. Metalloproteinase inhibitors for the disintegrin-like metalloproteinases ADAM10 and ADAM17 that differentially block constitutive and phorbol ester-inducible shedding of cell surface molecules. Comb Chem High Througput Screen. 2005;8(2):161-71.

27. Mattheij NJ, Swieringa F, Mastenbroek TG, Berny-Lang MA, May F, Baaten CC, et al. Coated platelets function in platelet-dependent fibrin formation via integrin allbb3 and transglutaminase factor XIII. Haematologica. 2016;101(4):427-36.

28. Schindelin J, Arganda-Carreras I, Frise E, Kaynig V, Longair M, Pietzsch T, et al. Fiji: an open-source platform for biological-image analysis. Nat Methods. 2012;9(7):676-82.

29. Andrews RK, Gardiner EE. Metalloproteolytic receptor shedding...platelets "acting their age". Platelets. 2016;27(6):512-8.

30. Rabie T, Strehl A, Ludwig A, Nieswandt B. Evidence for a role of ADAM17 (TACE) in the regulation of platelet glycoprotein V. J Biol Chem. 2005;280(15):14462-8.

31. Burkhart JM, M. V, Gambaryan S, Radau S, Walter U, Martens L, et al. The first comprehensive and quantitative analysis of human plalelet protein composition allows the comparative analysis of structural and functional pathways. Blood. 2012;120(15):e73-82.

32. Bevers EM, Comfurius P, Zwaal RF. Platelet procoagulant activity: physiological significance and mechanisms of exposure. Blood Rev. 1991;5(3):146-54.

33. Fong KP, Barry C, Tran AN, Traxler EA, Wannemacher KM, Tang HY, et al. Deciphering the human platelet sheddome. Blood. 2011;117(1):e15-26.

34. Mattheij NJ, Braun A, van Kruchten R, Castoldi E, Pircher J, Baaten CC, et al. Survival protein anoctamin-6 controls multiple platelet responses including phospholipid scrambling, swelling, and protein cleavage. FASEB J. 2016;30(2):727-37.

35. Munnix IC, Harmsma M, Giddings JC, Collins PW, Feijge MA, Comfurius P, et al. Store-mediated calcium entry in the regulation of phosphatidylserine exposure in blood cells from Scott patients. Thromb Haemost. 2003;89(4):687-95.

36. Gilio K, Harper MT, Cosemans JM, Konopatskaya O, Munnix IC, Prinzen L, et al. Functional divergence of platelet protein kinase C (PKC) isoforms in thrombus formation on collagen. J Biol Chem. 2010;285(30):234109.

37. van Kruchten R, Braun A, Feijge MAH, Kuijpers MJE, Rivera-Galdos R, Kraft P, et al. Antithrombotic potential of blockers of store-operated calcium channels in platelets. Arterioscler Thromb Vasc Biol. 2012;32(7):171723.

38. Agbani EO, van den Bosch MT, Brown E, Williams CM, Mattheij NJ, Cosemans JM, et al. Coordinated membrane ballooning and procoagulant spreading in human platelets. Circulation. 2015;132(15):1414-24.

39. Hofmann S, Vögtle T, Bender M, Rose-John S, Nieswandt B. The SLAM family member CD84 is regulated by ADAM10 and calpain in platelets. J Thromb Haemost. 2012;10(12):2581-92.

40. Bender M, Stegner D, Nieswandt B. Model systems for platelet receptor shedding. Platelets. 2016;Epub ahead of print:1-8.

41. Andrews RK, Gardiner EE. Basic mechanisms of platelet receptor shedding. Platelets. 2016;Epub ahead of print:1-6.

42. Sahin U, Weskamp G, Kelly K, Zhou HM, Higashiyama S, Peschon J, et al. Distinct roles for ADAM10 and ADAM17 in ectodomain shedding of six EGFR ligands. J Cell Biol. 2004;164(5):769-79.

43. Facey A, Pinar I, Arthur JF, Qiao J, Jing J, Mado B, et al. A-Disintegrin-And-Metalloproteinase (ADAM) 10 activity on resting and activated platelets. Biochemistry. 2016;55(8):1187-94.

44. Hantgan RR, Hindriks G, Taylor RG, Sixma JJ, de Groot PG. Glycoprotein Ib, von Willebrand factor, 
and glycoprotein IIb:Illa are all involved in platelet adhesion to fibrin in flowing whole blood. Blood. 1990;76(2):345-53.

45. Mammadova-Bach E, Ollivier V, Loyau S, Schaff M, Dumont B, Favier R, et al. Platelet glycoprotein VI binds to polymerized fibrin and promotes thrombin generation. Blood. 2015;126(5):683-91.

46. Alshehri OM, Hughes CE, Montague S, Watson SK, Frampton J, Bender M, et al. Fibrin activates GPVI in human and mouse platelets. Blood. 2015;126(13):1601-8.

47. de Cristofaro R, de Candia E, Croce G, Morosetti R, Landolfi R. Binding of human alpha-thrombin to platelet Gplb: energetics and functional effects. Biochem J. 1998;332(Pt 3):643-50.

48. Ravanat C, Strassel C, Hechler B, Schuhler S, Chicanne G, Payrastre B, et al. A central role of GPIb-IX in the procoagulant function of platelets that is independent of the $45-\mathrm{kDa}$ GPIba N-terminal extracellular domain. Blood. 2010;116(7):1157-64.

49. Béguin S, Keularts I, Al Dieri R, Belluci S, Caen J, Hemker HC. Fibrin polymerization is crucial for thrombin generation in platelet-rich plasma in a VWF-GPIb-dependent process, defective in Bernard-Soulier syndrome. J Thromb Haemost. 2004;2(1):170-6. 


\section{Supplemental data}

\section{Supplemental materials and methods:}

\section{Materials}

2-aminoethyldiphenylborinate (2APB), apyrase, bovine serum albumin (BSA), carbonyl cyanide 3-chlorophenylhydrazone (CCCP), dimethylsulfoxide, phorbol myristate acetate (PMA) and $\alpha$-thrombin were obtained from Sigma (St. Louis, MO, USA). Collagen related peptide cross-linked (CRP-XL) was purchased from Prof. Richard Farndale (University of Cambridge, UK) and convulxin was obtained from Stago BNL (Leiden, the Netherlands). ABT-737, 2MeS-ADP and thapsigargin were from SantaCruz Biotechnology (Santa Cruz, CA). Fluorescein isothiocyanate (FITC)-labeled monoclonal anti-GPIb $\alpha$ antibody, recognizing the extracellular domain, was acquired from Sanquin (Clone CLB-MB45), (Amsterdam, the Netherlands), phycoerythrin (PE)-labeled monoclonal anti-GPVI antibody, recognizing the ligand-binding ectodomain domain (residues 21234), from Biocytex (Marseille, France), PE-labeled monoclonal anti-GPIba antibody, recognizing GPIb $\alpha$ and glycocalicin, from DAKO (Heverlee, Belgium), FITC-labeled antiVWF antibody from Affinity Biologicals (Ancaster, Canada), and FITC-labeled monoclonal anti-GPIX antibody from eBioscience (San Diego, CA, USA). Fab fragment 5 G6 against GPIb $\alpha$, blocking the ADAM17-mediated cleavage of this chain was purified, as described before. ${ }^{1}$ Annexin A5 Alexa Fluor (AF)647-conjugated was from Invitrogen (Bleiswijk, the Netherlands), collagen type I from Nycomed Pharma (Munich, Germany) QVD-Oph, Ro318425 and ionomycin were from Calbiochem (San Diego, CA, USA). MDL-28170 was purchased from Tocris (Bristol, UK), 3,3'-dihexyloxacarbocyanine iodide (DiOC ${ }_{6}$ ) from Anaspec (Fremont, CA, USA), GW280264X from Aobious (Gloucester, MA, USA), and dimethyl-BAPTA acetoxymethyl ester (dm-BAPTA AM) from Molecular Probes (Waltham, MA, USA). Human prothrombin and factor Xa were purified and labeled using Oregon Green (OG)-488, as described. ${ }^{2-4}$ The active-site labeled coagulation factors had retained normal binding properties, while protease activity was fully blocked. ${ }^{2,3}$

\section{Platelet isolation}

PRP was prepared by centrifugation at $260 \mathrm{~g}$ for $15 \mathrm{~min}$. After a second centrifugation step, platelets were resuspended in Hepes buffer pH $6.6(10 \mathrm{mM}$ Hepes, $136 \mathrm{mM} \mathrm{NaCl}$, $2.7 \mathrm{mM} \mathrm{KCl}, 2 \mathrm{mM} \mathrm{MgCl}, 5 \mathrm{mM}$ glucose and $0.1 \% \mathrm{BSA}$ ). Platelet suspensions were re-centrifuged in the presence of 1:15 $A C D$ and $1 \mathrm{U} / \mathrm{mL}$ apyrase and subsequently resuspended into Hepes buffer pH 7.45 (10 mM Hepes, 136 mM NaCl, 2.7 mM KCl, 2 mM $\mathrm{MgCl}_{2}, 5 \mathrm{mM}$ glucose and $\left.0.1 \% \mathrm{BSA}\right)$. 


\section{Supplemental table and figures:}

Table S1: Combined effects of agonists and inhibitors on glycoprotein shedding. Washed platelets were pre-treated for 15 min with the ADAM10/17 inhibitor GW280264X (5 $\mu \mathrm{M}),{ }^{5}$ the calpain inhibitor MDL-28170 $(200 \mu \mathrm{M}),{ }^{6}$ the pan-caspase inhibitor QVD-OPh $(10 \mu \mathrm{M}),{ }^{7}$ the general PKC inhibitor Ro-318425 $(10 \mu \mathrm{M}),{ }^{8}$ the calcium chelator dm-BAPTA AM $(20 \mu \mathrm{M}),{ }^{7}$ or the $\mathrm{Ca}^{2+}$ entry inhibitor $2 \mathrm{APB}(100 \mu \mathrm{M}) .{ }^{9}$ Platelet samples were then stimulated with ionomycin/ $\mathrm{CaCl}_{2}(10 \mu \mathrm{M} / 2 \mathrm{mM}), \mathrm{CRP}-\mathrm{XL} /$ thrombin $(5 \mu \mathrm{g} / \mathrm{mL} / 4 \mathrm{nM}), \mathrm{PMA}(100 \mathrm{nM})$, ABT-737 $(10 \mu \mathrm{M})$ or CCCP $(100 \mu \mathrm{M})$, as indicated. For the whole platelet population, surface expression of GPIba (mean fluorescence intensity) was measured after stimulation for $60 \mathrm{~min}$ (ionomycin, ABT-737) or 180 min (CRP-XL/thrombin, PMA, CCCP). Data were normalized to the values of unstimulated platelets without any inhibitors. Means $\pm S D, n=3-5 ;{ }^{*} p<0.05$ vs. no inhibitor.

${ }^{8} \mathrm{CaCl}_{2}$ omitted instead.

\begin{tabular}{|l|l|l|l|l|l|l|}
\hline $\begin{array}{l}\text { Inhibition or } \\
\text { chelation of }\end{array}$ & $\begin{array}{l}\text { Unstimu- } \\
\text { lated }\end{array}$ & lonomycin & $\begin{array}{l}\text { CRP-XL + } \\
\text { Thr }\end{array}$ & PMA & ABT-737 & CCCP \\
\hline Control & 1.00 & $0.08 \pm$ & $0.17 \pm$ & $0.40 \pm$ & $0.09 \pm$ & $0.64 \pm$ \\
& & 0.02 & 0.07 & 0.12 & 0.06 & 0.14 \\
\hline ADAM10/17 & $0.99 \pm$ & $0.67 \pm$ & $0.79 \pm$ & $0.79 \pm$ & $0.85 \pm$ & $1.01 \pm$ \\
& 0.08 & $0.04 *$ & $0.12 *$ & $0.14 *$ & $0.10 *$ & $0.10 *$ \\
\hline Calpain & $0.94 \pm$ & $0.24 \pm$ & $0.35 \pm$ & $0.38 \pm$ & $0.12 \pm$ & $0.45 \pm$ \\
& 0.09 & $0.04^{*}$ & 0.11 & 0.06 & 0.01 & 0.13 \\
\hline Caspases & $0.99 \pm$ & $0.08 \pm$ & $0.22 \pm$ & $0.45 \pm$ & $0.87 \pm$ & $0.73 \pm$ \\
& 0.01 & 0.02 & 0.04 & 0.10 & $0.17 *$ & 0.19 \\
\hline PKC & $1.01 \pm$ & $0.04 \pm$ & $0.38 \pm$ & $0.96 \pm$ & $0.11 \pm$ & $0.50 \pm$ \\
& 0.08 & 0.01 & $0.11 *$ & $0.10 *$ & 0.03 & 0.12 \\
\hline Calcium & $0.85 \pm$ & $0.87 \pm$ & $0.70 \pm$ & $0.52 \pm$ & $0.52 \pm$ & $0.72 \pm$ \\
& 0.05 & $0.02 * \S$ & $0.12 *$ & 0.20 & $0.17 *$ & 0.07 \\
\hline Calcium entry & $0.92 \pm$ & $0.87 \pm$ & $0.59 \pm$ & $0.24 \pm$ & $0.13 \pm$ & $0.42 \pm$ \\
& 0.13 & $0.02 * \S$ & $0.05 *$ & 0.07 & 0.05 & 0.14 \\
\hline
\end{tabular}



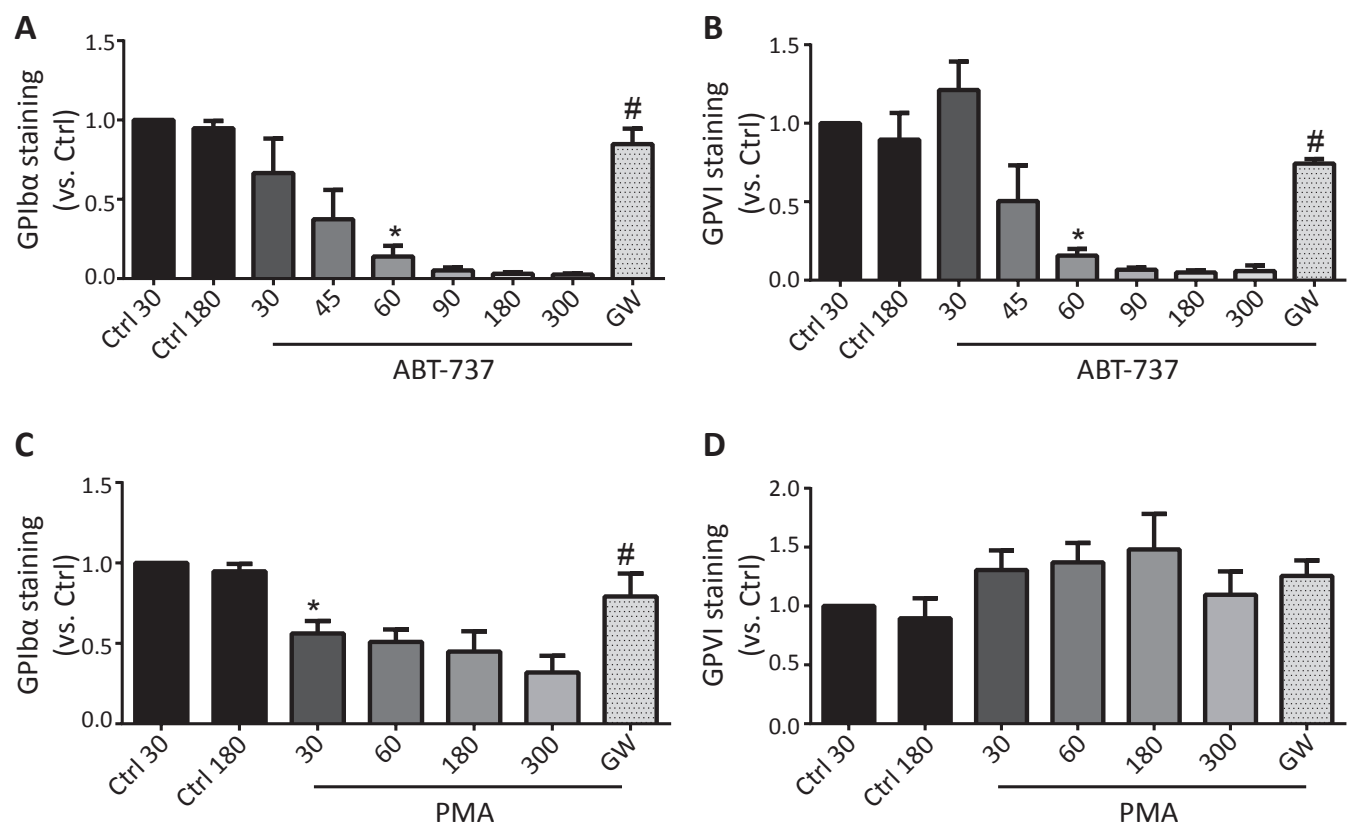

$\mathbf{E}$

$\mathbf{F}$
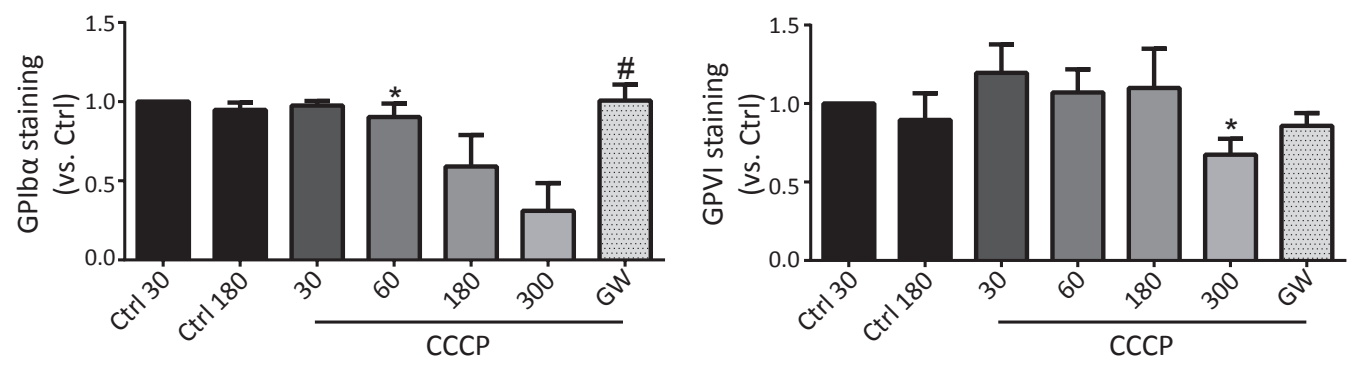

Figure S1: Differential glycoprotein receptor shedding after platelet stimulation with ABT-737, PMA or CCCP. Washed platelets, pre-incubated with DMSO (vehicle) or GW280264X (GW, $5 \mu \mathrm{M}$ ), were stimulated with near-maximal concentrations of ABT-737 (A-B), PMA (C-D) or CCCP (E-F) for indicated times (30-300 min) at $37^{\circ} \mathrm{C}$. Unstimulated platelets were used as control (Ctrl). Staining for GPIb $\alpha$ and GPVI was measured with fluorescent-labeled antibodies using flow cytometry (mean fluorescence intensities). Data were normalized to values of unstimulated platelets. Data are mean $\pm S D, n=3-7$ ( $n \geq 3$ donors); ${ }^{*} p<0.05$ vs. unstimulated control, ${ }^{\#} p<0.05$ vs. vehicle. 
A
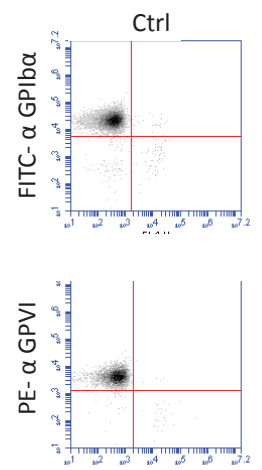

AF647-annexin A5

C
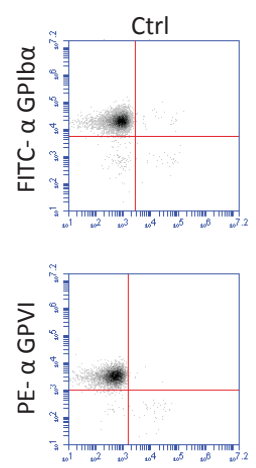

AF647-annexin A5
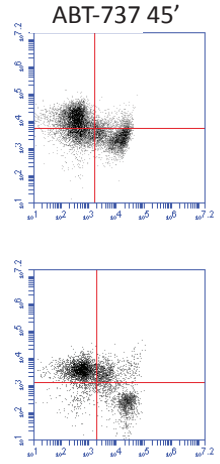

AF647-annexin A5
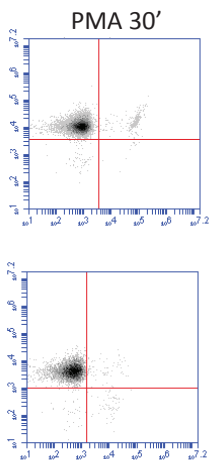

AF647-annexin A5
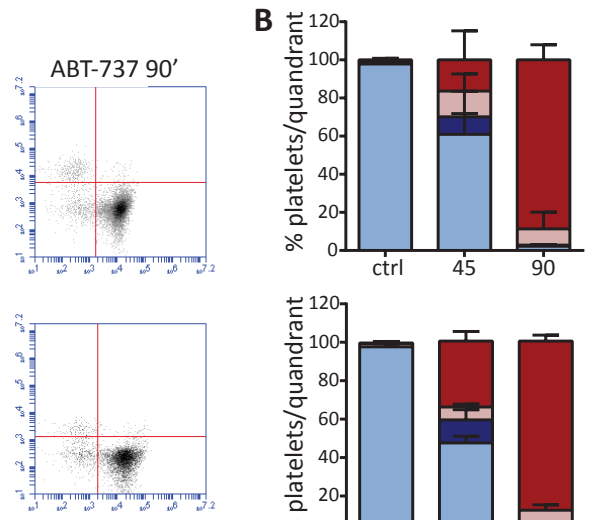

AF647-annexin A5

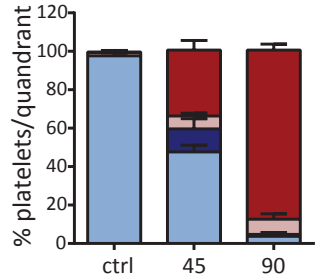

$\square$ GPVI low/PS+

$\square$ GPVI low/PS-

GPVI high/PS+

$\square$ GPVI high/PS-
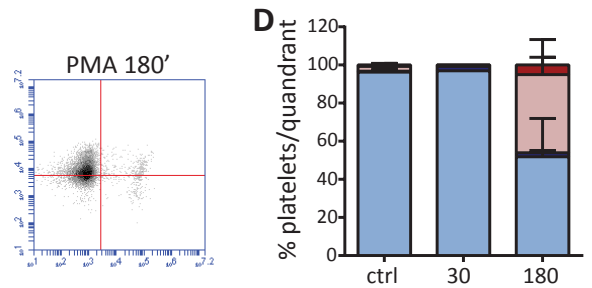

$\square$ GPIba low/PS+

$\square$ GPIba low/PS-

GPIba high/PS+

$\square$ GPIba high/PS-

Figure S2: Differential glycoprotein receptor shedding and PS exposure in platelets stimulated with ABT-737 or PMA. Washed platelets were stimulated with ABT-737 (A-B) or PMA (C-D) for indicated times, and evaluated for glycoprotein expression, as for Figure S1. Unstimulated platelets were used as control (Ctrl). Platelets were dually stained for GPIb $\alpha$ or GPVI expression and PS exposure (AF647-annexin A5) at indicated time points. A, Representative dot plots showing a gradual decrease in GPIba and GPVI expression accompanying PS exposure. B, Quantification of four quadrants of platelet populations, as in Figure 2. C, Representative dot plots showing a decrease in GPIb $\alpha$ but not GPVI expression with limited PS exposure. D, Quantification of platelet populations. Data are means $\pm S D, n=3-5$ ( $n \geq 3$ donors); ${ }^{*} p<0.05$. 

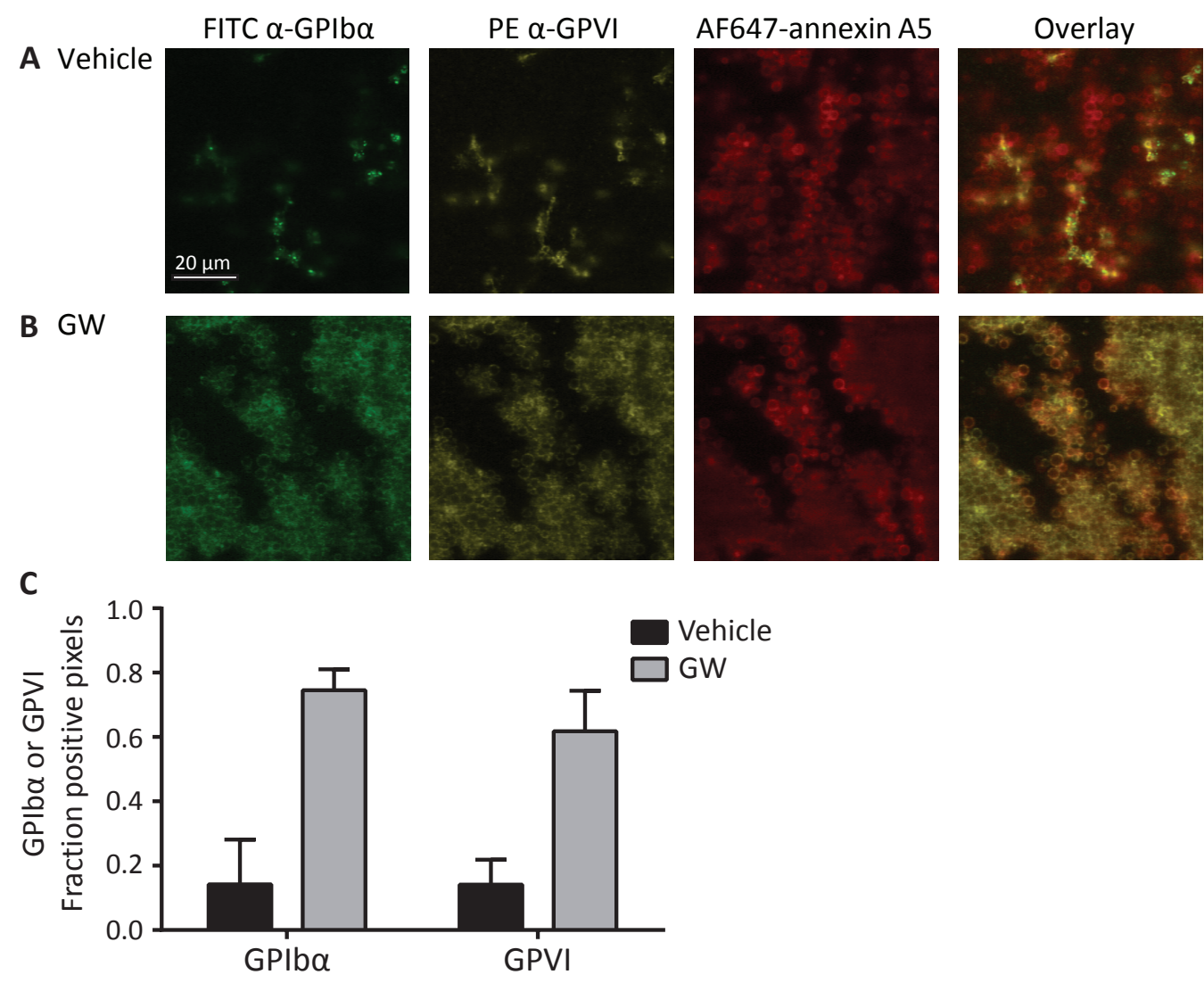

B GW

C
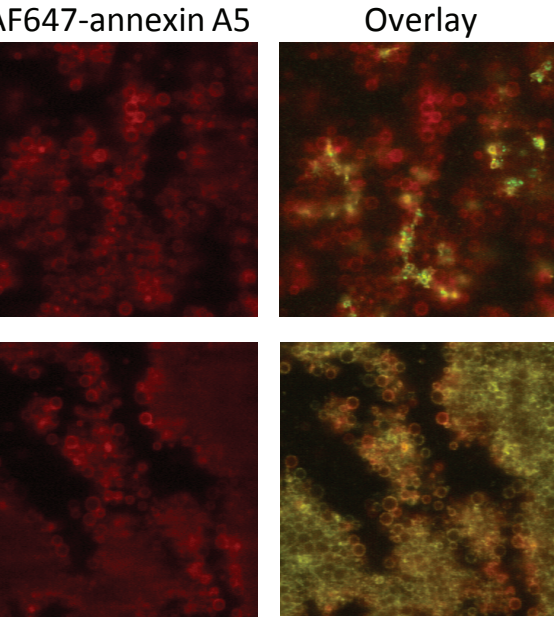

Figure S3: Massive glycoprotein shedding of platelets in thrombi induced by ionomycin. Thrombi were formed on collagen type under arterial flow conditions, and subsequently post-perfused with $10 \mu \mathrm{M}$ ionomycin and $5 \mathrm{mM} \mathrm{CaCl}$ for $5 \mathrm{~min}$ in the presence or absence of $\mathrm{GW} 280264 \mathrm{X}(\mathrm{GW}$ ). Platelets in thrombi were then stained for GPIb $\alpha$ and GPVI expression and PS exposure. Representative images of GPIb $\alpha$ and GPVI expression and PS exposure after ionomycin activation in the presence of vehicle $(\mathbf{A})$ or $\mathrm{GW}(\mathbf{B})$. Bar = $20 \mu \mathrm{m}$. Fractions of ballooned platelets staining for GPIba or GPVI (C). Means \pm SD; $\mathrm{n}=3,{ }^{*} p<0.05$. 

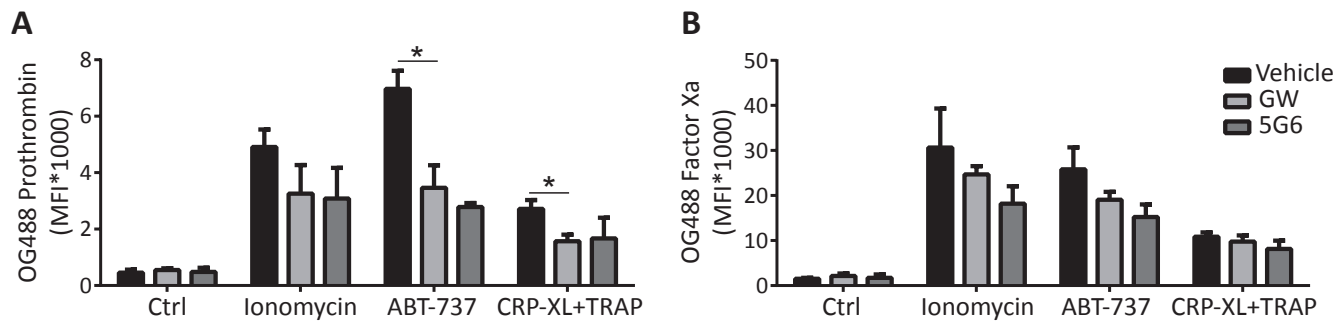

Figure S4: Prothrombin and factor $\mathrm{Xa}$ binding within the whole platelet population. Washed platelets were pre-incubated for $15 \mathrm{~min}$ with vehicle, GW280264X (GW, $5 \mu \mathrm{M})$ or the $5 \mathrm{G} 6$ Fab fragment $(10 \mu \mathrm{g} / \mathrm{mL})$, which blocks ADAM17-mediated shedding of GPIba. After $60 \mathrm{~min}$ of stimulation $\left(37^{\circ} \mathrm{C}\right)$ with ionomycin, ABT-737 or CRP-XL/SFLLRN, binding to platelets was measured of OG488-prothrombin (A) or OG488-factor Xa (B) for the whole platelet population using flow cytometry. Means $\pm S D ; n=3-4,{ }^{*} p<0.05$.

Collagen/thrombin

(ionomycin)

\section{high intracellular $\mathrm{Ca}^{2+}$}

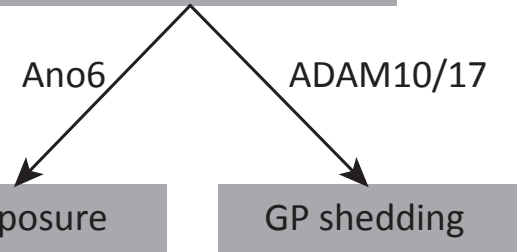

ABT-737

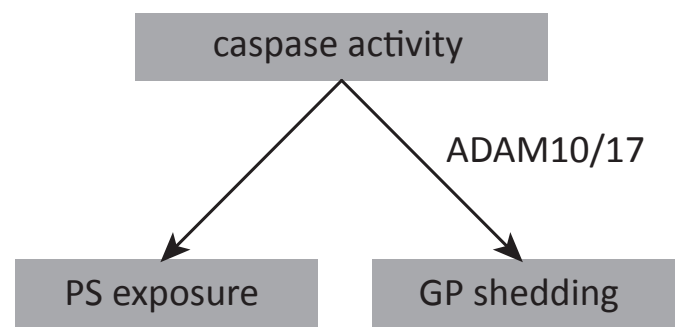

Figure S5: Schematic representation of intracellular pathways involved in both receptor shedding and PS exposure. Abbreviations: Ano6, Anoctamin 6. 


\section{References}

1. Liang X, Russell SR, Estelle S, Jones LH, Cho S, Kahn ML, et al. Specific inhibition of ectodomain shedding of glycoprotein Ib $\alpha$ by targeting its juxtamembrane shedding cleaving site. J Thromb Haemost. 2013;11(12):2155-62.

2. Bock PE. Active-site-selective labeling of blood coagulation proteinases with fluorescence probes by the use of thioester peptide chloromethyl ketones. II. Properties of thrombin derivatives as reporters of prothrombin fragment 2 binding and specificity of the labeling approach for other proteinases. J Biol Chem. 1992;1992(267):21.

3. Panizzi P, Friedrich R, Fuentes-Prior P, Kroh HK, Briggs J, Tans G, et al. Novel fluorescent prothrombin analogs as probes of staphylocoagulase-prothrombin interactions. J Biol Chem. 2006;281(2):1169-78.

4. Berny MA, Munnix IC, Auger JM, Schols SE, Cosemans JM, Panizzi P, et al. Spatial distribution of factor Xa, thrombin, and fibrin(ogen) on thrombi at venous shear. PLos One. 2010;5(4):e10415.

5. Donners MM, Wolfs IM, Olieslagers S, Mohammadi-Motahhari Z, Tchaikovski V, Heeneman S, et al. A disintegrin and metalloprotease 10 is a novel mediator of vascular endothelial growth factor-induced endothelial cell function in angiogenesis and is associated with atherosclerosis. Arterioscler Thromb Vasc Biol. 2010;30(11):2188-95.

6. Mattheij NJ, Gilio K, van Kruchten R, Jobe SM, Wieschhaus AJ, Chishti AH, et al. Dual mechanism of integrin $\alpha_{11 b} \beta_{3}$ closure in procoagulant platelets. J Biol Chem. 2013;288(19):13325-36.

7. van Kruchten R, Mattheij NJ, Saunders C, Feijge MAH, Wolfs JL, Collins PW, et al. Both TMEM16Fdependent and TMEM16F-independent pathways contribute to phosphatidylserine exposure in platelet apoptosis and platelet activation. Blood. 2013;121(10):1850-7.

8. Gilio K, Harper MT, Cosemans JM, Konopatskaya O, Munnix IC, Prinzen L, et al. Functional divergence of platelet protein kinase C (PKC) isoforms in thrombus formation on collagen. J Biol Chem. 2010;285(30):234109.

9. van Kruchten R, Braun A, Feijge MAH, Kuijpers MJE, Rivera-Galdos R, Kraft P, et al. Antithrombotic potential of blockers of store-operated calcium channels in platelets. Arterioscler Thromb Vasc Biol. 2012;32(7):171723. 



\section{Chapter 6}

Impaired mitochondrial activity explains platelet dysfunction in thrombocytopenic cancer patients undergoing chemotherapy

Baaten CC, Moenen FC*, Henskens $\mathrm{YM}^{*}$, Swieringa F, Wetzels R, van Oerle R, Heijnen HF, Ten Cate H, Holloway GP, Beckers EA, Heemskerk JW, van der Meijden PE (*equal contribution)

Haematologica. 2018; major revision 


\section{Abstract}

Severe thrombocytopenia $\left(\leq 50 \times 10^{9}\right.$ platelets/L) due to hematological malignancy and intensive chemotherapy is associated with an increased risk of clinically significant bleeding. Since the bleeding risk is not linked to the platelet count only, other hemostatic factors must be involved. In 70 patients with acute leukemia, multiple myeloma or malignant lymphoma, who experienced chemotherapy-induced thrombocytopenia, we studied both platelet and coagulant functions, and furthermore evaluated effects of platelet transfusion. Platelets from all patients - independent of disease or treatment type - were to a variable extent compromised in $\mathrm{Ca}^{2+}$ flux, integrin $\alpha_{1 \mathrm{Ib}} \beta_{3}$ activation and P-selectin expression, when stimulated with a panel of receptor agonists. The patients' platelets were also impaired in spreading on fibrinogen. Whereas the $\mathrm{Ca}^{2+}$ store content was unaffected, the patients' platelets showed ongoing phosphatidylserine (PS) exposure, which was not due to apoptotic caspase activity. Mitochondrial function was markedly reduced in the patients' platelets, as evidenced by a low mitochondrial membrane potential $(p<0.001)$ and low oxygen consumption $(p<0.05)$, while the mitochondrial content was normal. Whole blood platelet adhesion and fibrin formation under flow were markedly impaired, and increased after transfusion with platelet concentrate. Plasma coagulation factors were in the normal range, except for a marginal reduction in factor VII. In conclusion, our findings disclose a defective receptor signaling related to impaired mitochondrial bioenergetics, independent of apoptosis, in platelets from cancer patients treated with chemotherapy, explaining the low hemostatic potential of these patients.

\section{Introduction}

Platelets are indispensable for maintaining vascular integrity and accomplishing hemostatic plug formation. ${ }^{1} \mathrm{~A}$ sufficient platelet count as well as an adequate platelet function is required for prevention of bleeding. Patients with hematological malignancies, such as leukemia, multiple myeloma or malignant lymphoma, are commonly treated with combination chemotherapy, frequently followed by bone marrow transplantation. This treatment impairs proliferation of megakaryocytes and ensuing production of proplatelets. As a consequence, severe thrombocytopenia, i.e. a platelet count of $\leq 50 \times 10^{9} / \mathrm{L}$, develops in essentially all treated patients. ${ }^{2}$ Prophylactic transfusion with platelet concentrates for preventing bleeding is given as standard care once the count drops below $10 \times 10^{9} / \mathrm{L}$ or in case of active bleeding. ${ }^{2,3}$ Randomized clinical trials have indicated that the bleeding risk in this patient group is reduced by platelet transfusion, although it does not completely eliminate hemorrhagic events. ${ }^{4,5}$ Since bleeding is relatively infrequent in non-malignant thrombocytopenia, 6,7 it can be considered that a low platelet count is not the sole risk factor for bleeding in chemotherapy-treated patients.

Earlier studies on patients with acute myeloid leukemia, not receiving chemotherapy, have provided indications for impaired platelet function due to disease, as apparent from low platelet aggregation, reduced granule secretion and weak thromboxane $B_{2}$ production. ${ }^{8-10}$ It was proposed that low expression of the $\alpha$-granule glycoprotein, P-selectin, can be used as a prognostic marker for hemorrhage. ${ }^{11}$ However, bleeding in combination with thrombocytopenia is more frequently observed in cancer 
patients treated with chemotherapy. ${ }^{12}$ The literature so far only indicates that the anthracycline daunorubicin inhibits integrin $\alpha_{11 b} \beta_{3}$ activation, aggregation and secretion of platelets upon agonist stimulation. ${ }^{13,14}$ Daunorubicin and its analogue idarubicin were found to induce integrin activation and secretion in resting platelets. ${ }^{15}$ However, to which extent and by which mechanism myelosuppressive chemotherapy in general affects platelet function has remained largely unclear.

In the present study, we evaluated the platelet activation processes and coagulant activity in 70 patients with hematological malignancies treated with chemotherapy. Our results point to multiple functional defects in the patients' platelets that are related to impaired mitochondrial activity, independent of classical apoptosis. In the majority of the patients, low platelet activity could be improved by platelet transfusion.

\section{Methods}

\section{Materials and methods}

See Data Supplement.

\section{Patients and control subjects}

The study was approved by the local ethics committee (METC-11-4-097). All participating patients and healthy volunteers gave written informed consent according to the Helsinki declaration. Patients, reporting at the hospital, fulfilling the inclusion criteria and providing informed consent, were consecutively included in the period of November 2014 until January 2017. Eligible patients were $\geq 18$ years of age, received chemotherapy for treatment of a confirmed hematologic malignancy (acute myeloid leukemia, acute lymphoid leukemia, multiple myeloma or malignant lymphoma), and had or were expected to have thrombocytopenia (platelet count $\leq 50 \times 10^{6} / \mathrm{mL}$ ). Morning platelet counts were monitored daily as part of the routine clinical care. According to standard practice, when the morning platelet count was $<10 \times 10^{6} / \mathrm{mL}$, patients received prophylactic transfusion with one batch of platelet concentrate (leukocyte-depleted pooled buffy coat from 5 donors, median storage time: 6 days, median platelet count: 357 $\mathrm{x} 10^{6} / \mathrm{mL}$ ). Patient exclusion criteria were: sepsis, splenomegaly, signs of active bleeding at the time of blood withdrawal, previous platelet transfusion within 3 days, and/or use of antithrombotic medication in the past 14 days.

For clinical care, blood samples were collected before and during chemotherapeutic treatment at multiple time points: 1) before myelosuppression, 2) during myelosuppression (platelet count $\leq 50 \times 10^{6} / \mathrm{mL}$ ), 3) during myelosuppression: before (platelet count $\leq 10 \times 10^{6} / \mathrm{mL}$ ) and $1 \mathrm{~h}$ after platelet transfusion and 4 ) during bone marrow recovery (platelet count $\leq 50 \times 10^{6} / \mathrm{mL}$ ). Patient blood samples were obtained via a central venous catheter, rinsed with $100 \mathrm{~mL}$ saline to remove residual traces of heparin (verified by measurement of thrombin time). Blood samples from healthy control subjects were obtained via venipuncture of the antecubital vein using a Vacutainer 21-gauge needle (Becton-Dickinson Bioscience, Franklin Lakes, NJ). Blood collection was always into $3.2 \%$ $(w / v)$ trisodium citrate (Greiner Bio-One Vacuette, Alphen a/d Rijn, The Netherlands). 
For clinical care (hematological parameters), separate samples from patients were drawn into vacuette tubes containing $\mathrm{K}_{2}$-EDTA (Becton-Dickinson Bioscience, Franklin Lakes, NJ, USA).

\section{Experimental setup}

Within the limitations of medical ethical permission, in total blood samples from 52 patients (platelet count $\leq 50 \times 10^{6} / \mathrm{mL}$ ) could be obtained during myelosuppression (study A). In all these samples, platelet responsiveness was assessed using flow cytometry. Due to the limited blood volume and the low platelet counts, a restricted number of additional analyses was carried out per sample. When sufficient sample volume was remaining, platelet function was further characterized by measuring the following platelet responses: platelet spreading, intracellular calcium signaling and PS exposure. To gain a deeper understanding of the underlying mechanisms of platelet dysfunction, subsequently blood samples could be obtained from 18 additional patients (platelet count $\leq 50 \times 10^{6} / \mathrm{mL}$ ) during the myelosuppression phase (study B). The samples were used to investigate apoptotic signaling (caspase activity; western blotting for caspasemediated protein cleavage) or mitochondrial respiration and structure (high-resolution respirometry, citrate synthase activity, transmission electron microscopy). Care was taken that for all measurements patients from the major treatment classes were represented (see figure legends).

For 36 of the patients of study A, blood samples could also be obtained at $1 \mathrm{~h}$ after transfusion with platelet concentrate. Again, platelet responsiveness was determined by flow cytometry. In case of sufficient blood volume, whole blood thrombus and fibrin formation under flow were assessed in the pre- and post-transfusion samples.

\section{Statistical analysis}

Data are represented as medians with interquartile ranges. Paired data were compared using the Wilcoxon signed-rank test, otherwise the Mann-Whitney $\mathrm{U}$ test was used. When comparing more than two groups, the Kruskal Wallis $\mathrm{H}$ test was used. P-values $<0.05$ were considered significant. Graphs were made using Graphpad Prism v6 (San Diego, CA, USA). Statistical analysis was performed using the SPSS Statistics 23 package (IBM, Armonk, NY, USA).

\section{Results}

Variable impairment of platelet activation in cancer patients with thrombocytopenia after chemotherapy

Blood samples were obtained from in total 70 patients, who were diagnosed with acute myeloid leukemia or acute lymphocytic leukemia (AML/ALL, $n=36$ ), multiple myeloma ( $n=16)$, malignant lymphoma $(n=14)$ or other hematologic malignancies $(n=4)$. 
Table 1: Characteristics and hematological parameters of patients during myelosuppression. Data are for total number of patients $n=70$. Patient information for study A $(n=52)$ is indicated between brackets. Median values are given.

\begin{tabular}{|c|c|c|}
\hline Patient characteristics & \multicolumn{2}{|l|}{ Number /Value } \\
\hline Age (years) & \multicolumn{2}{|l|}{$60(60)$} \\
\hline Female/male (n) & \multicolumn{2}{|l|}{$28 / 42(20 / 32)$} \\
\hline $\begin{array}{l}\text { Diagnosis (n) } \\
\text { - AML/ALL } \\
\text { - Multiple Myeloma } \\
\text { - Lymphoma } \\
\text { - Other }\end{array}$ & \multicolumn{2}{|l|}{$\begin{array}{l}36(25) \\
16(12) \\
14(13) \\
4(2)\end{array}$} \\
\hline $\begin{array}{l}\text { Stem cell transplantation ( } n \text { ) } \\
\text { - Autologous } \\
\text { - Allogeneic }\end{array}$ & \multicolumn{2}{|l|}{$\begin{array}{l}32(26) \\
11(8)\end{array}$} \\
\hline Time since chemotherapy (days) & \multicolumn{2}{|l|}{$8(9)$} \\
\hline $\begin{array}{l}\text { Time since stem cell transplanta- } \\
\text { tion (days) }\end{array}$ & \multicolumn{2}{|l|}{$8(8)$} \\
\hline Blood parameters & Value & Reference range \\
\hline Leucocyte count (x 109/L) & $0.26(0.22)$ & $3.5-11.0$ \\
\hline Hemoglobin (mM) & $5.7(5.7)$ & $7.5-11.0$ \\
\hline Platelet count (x 109/L) & $8(7)$ & $150-400$ \\
\hline $\begin{array}{l}\text { Absolute immature platelet num- } \\
\text { ber }\left(\times 10^{9} / \mathrm{L}\right)\end{array}$ & $0.31(0.26)$ & \\
\hline Immature platelet fraction (\%) & $3.9(3.6)$ & $1.1-6.1^{44}$ \\
\hline
\end{tabular}

All patients experienced severe thrombocytopenia due to chemotherapy, which was stopped at median 8 days before blood sample analysis (Table 1). The median age of the patient group was 60 years, and $40 \%$ was female (Table 1). Leukocyte and platelet counts were below normal, as was the hemoglobin level.

Treatment regimens in accordance with national guidelines varied with disease type. ${ }^{16-18}$ Since these regimens consisted of multiple chemotherapeutic compounds, the distribution of the drugs was evaluated among patients with different diagnoses. Therefore, the various drugs were assigned to one of five pharmacological classes: A, antitumor antibiotics \& topo-isomerase II inhibitors; B, antimetabolites; C, alkylating agents; D, mitotic inhibitors; $\mathrm{E}$, other (Table S1) ${ }^{19}$ Most patients appeared to be treated with anti-tumor antibiotics/topo-isomerase inhibitors, antimetabolites and/or alkylating agents (Table S2). The patients diagnosed with AML/ALL and lymphoma usually received drugs from one or more of these three classes, while the patients diagnosed with multiple myeloma only obtained alkylating agents. Of all 70 patients, 43 had undergone hematopoietic stem cell transplantation before inclusion, of which 32 patients received 
an autologous transplant and 11 an allogenic (Table 1). Blood samples were obtained at 8 days (median) after the last administration of chemotherapy or at 8 days (median) after stem cell transplantation.

Responsiveness of washed platelets was determined by flow cytometry, using a platelet count of $10 \times 10^{6} / \mathrm{mL}$, for 52 patients and 27 healthy control subjects. In the absence of agonists, surface activation markers were low for both patient and control platelets. After stimulation with ADP (P2Y ${ }_{1 / 12}$ agonist), CRP-XL (GPVI agonist) or thrombin

A

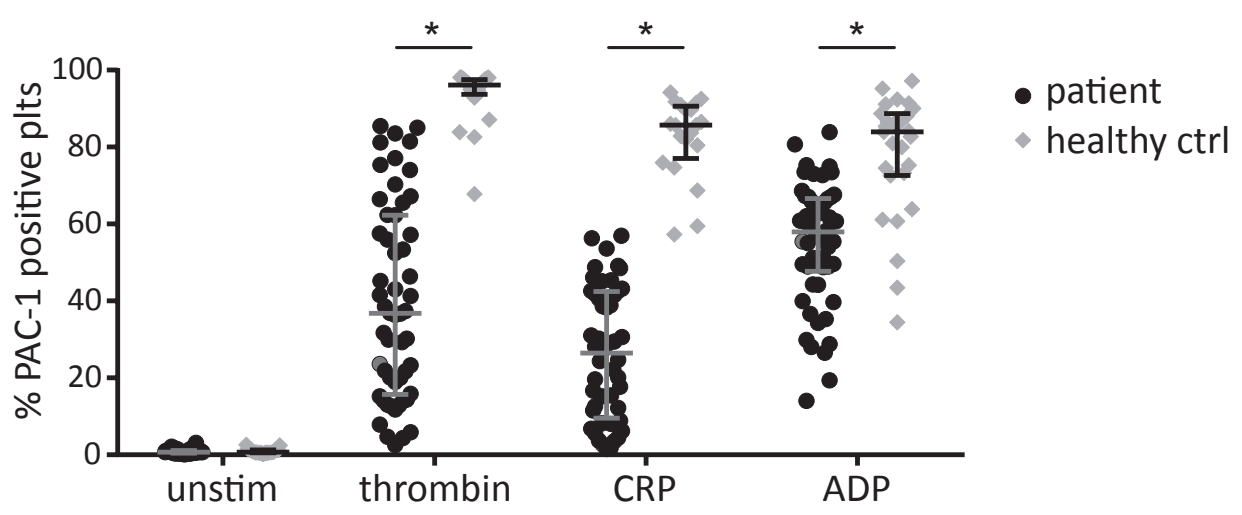

B

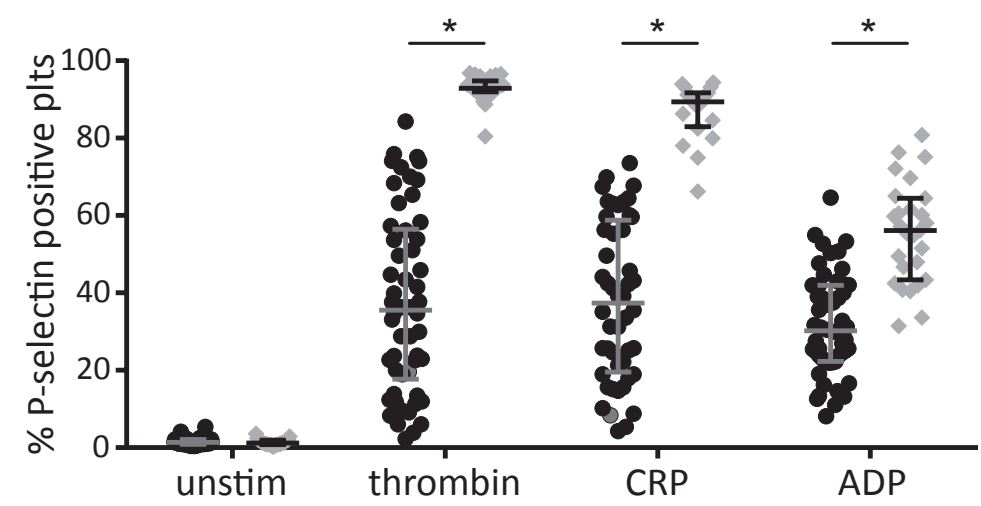

Figure 1: Variable impairment of integrin $\alpha_{11 \mathrm{~b}} \beta_{3}$ activation and P-selectin expression in stimulated platelets from cancer patients with thrombocytopenia after chemotherapy.Washed platelets $\left(10 \times 10^{6} / \mathrm{mL}\right) \mathrm{from}$ healthy control subjects (healthy ctrl) and thrombocytopenic patients receiving chemotherapy were activated with thrombin $(4 \mathrm{nM}), \mathrm{CRP}-\mathrm{XL}(10 \mu \mathrm{g} / \mathrm{mL})$ or $2 \mathrm{MeS}-\mathrm{ADP}(1 \mu \mathrm{M})$ in the presence of $2 \mathrm{mM} \mathrm{CaCl}_{2}$. After 15 min activation, integrin $\alpha_{\| 1 b} \beta_{3}$ activation (A) and P-selectin expression (B) were measured by flow cytometry using PAC-1 and anti-P-selectin antibody, respectively. Medians with interquartile ranges (IQR); data from 52 patients ( $25 \mathrm{AML} / \mathrm{ALL}, 12$ multiple myeloma, 13 lymphoma, 2 other), 27 healthy controls. $* p$ $<0.05$. 

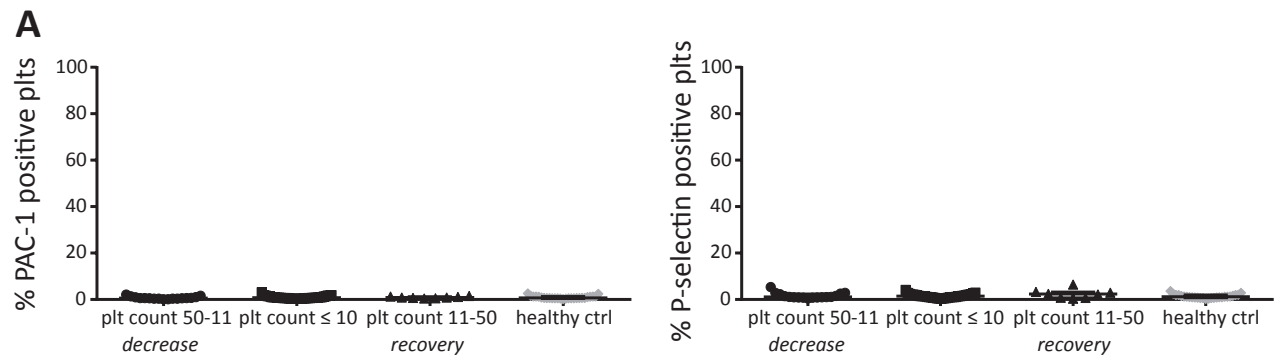

B
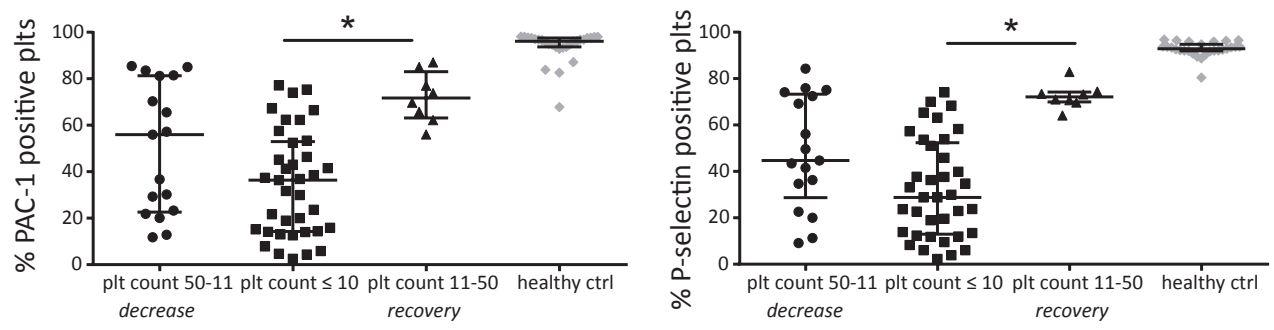

C
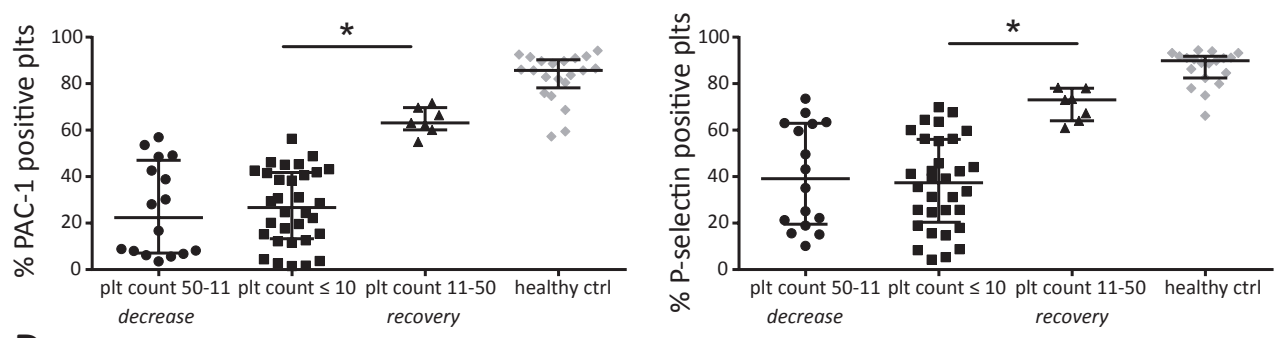

D
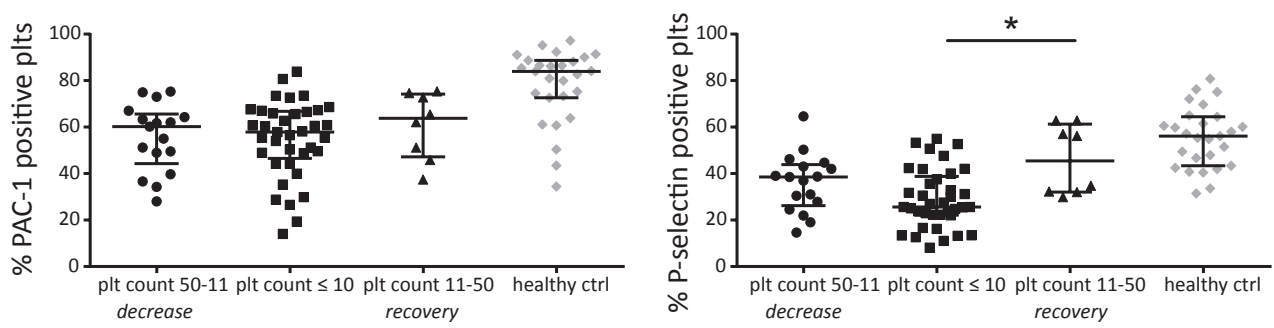

Figure 2: Impaired platelet responsiveness in relation to phase of treatment and/or recovery. Platelet integrin $\alpha_{11 b} \beta_{3}$ activation and P-selectin expression were measured (see Figure 1 ). Patients $(n=52)$ were divided into two categories: (i) decreasing platelet count 50-11 $\times 10^{6} / \mathrm{mL}(n=15)$ and (ii) decreasing platelet count $\leq 10 \times 10^{6} / \mathrm{mL}(n=37)$. Furthermore, from a subset of patients a sample could be collected when the platelet count increased independently of platelet transfusion (iii): $\left.11-50 \times 10^{6} / \mathrm{mL}(n=8)\right)$. Data are expressed as \% of platelets positive for PAC-1 or anti-P-selectin staining in the absence of stimulation (A), or after stimulation with thrombin (B), CRP-XL (C) or 2MeS-ADP (D). Medians with IQR for patients and healthy controls $(n=27) ;{ }^{*} p<0.05$. 
(PAR1/4 agonist) at maximal doses, integrin $\alpha_{111} \beta_{3}$ activation (Figure 1A) and P-selectin expression (Figure 1B) of the patients' platelets were reduced to a variable extent, when compared to the controls, irrespective of the agonist used.

Detailed analysis indicated that the overall platelet responsiveness (median=36.8\%, IQR=29.7- 46.7\%), defined as the average fraction of platelets positive for integrin activation and P-selectin expression for the three agonists: (i) was not different between diagnoses, i.e. AML/ALL, multiple myeloma, lymphoma and other hematological malignancies (Kruskal Wallis $\mathrm{H}$ test, $p=0.192$ ); (ii) was not affected by stem cell transplantation, i.e. no transplant, autologous or allogenic stem cell transplantation (Kruskal Wallis $\mathrm{H}$ test, $p=0.640$ ); (iii) was similar for the four major treatment classes, i.e. $\mathrm{A}+\mathrm{B}, \mathrm{A}+\mathrm{B}+\mathrm{C}, \mathrm{B}+\mathrm{C}, \mathrm{C}$ (Kruskal Wallis $\mathrm{H}$ test, $p=0.512$; Figure S1); and (iv) did not correlate with the whole blood platelet count (Spearman's $r h o=0.175, p=0.239$ ). Together, this suggested that the variability in platelet responsiveness among patients was not directly linked to the disorder, treatment type or number of (residual) circulating platelets. Additional functional analyses were performed with platelets, always from patients in the major treatment classes.

\section{Impaired platelet responsiveness during myelosuppression}

To determine whether the reduced platelet responsiveness was linked to the treatment phase, flow cytometric analysis of platelet responsiveness was performed during the decreasing period of platelet count $\left(50-11 \times 10^{6} / \mathrm{mL}\right.$ and $\left.\leq 10 \times 10^{6} / \mathrm{mL}\right)$ and the recovery of platelet count $\left(11-50 \times 10^{6} / \mathrm{mL}\right)$. The latter was defined as a sustained increase in platelet count (observed for patient care), independent of platelet transfusion. Of the eight patients included in this category, three had received an autologous transplant and one patient an allogeneic stem cell transplant, prior to recovery. In the decreasing period, integrin activation and P-selectin expression following stimulation with thrombin or CRP-XL were comparable in patients with platelet counts in the range of 50-11 $\times 10^{6} /$ $\mathrm{mL}$ and $\leq 10 \times 10^{6} / \mathrm{mL}$ (Figure 2). In contrast, platelet responsiveness to thrombin and CRP-XL significantly improved in case of count recovery $(p<0.001)$. For stimulation with $A D P$, these differences were less pronounced, with only P-selectin expression increased during count recovery. These results indicated that platelet count alone is not a good marker of platelet activity.

For five patients ( $1 \mathrm{AML}, 3$ multiple myeloma, 1 lymphoma), blood samples could also be analyzed at an earlier time point, i.e. after the stop of chemotherapy, but before severe thrombocytopenia occurred. Remarkably, in all these samples, platelet function was within normal range for the three agonists (integrin activation 69-86\%, P-selectin expression 49-85\%). This argued against a direct effect of the chemotherapeutics on the platelet activation properties.

Impaired platelet spreading and $\mathrm{Ca}^{2+}$ signaling of platelets after chemotherapy treatment

To further characterize the patient platelets, they were allowed to adhere and spread for $10 \mathrm{~min}$ on a fibrinogen surface, interacting with platelet integrin $\alpha_{11 \mathrm{~b}} \beta_{3}$. The observed morphology of the cells was divided into three stages: 1) formation of one or more filopodia, 2) formation of filopodia and lamellipods, and 3) full spreading. Most 


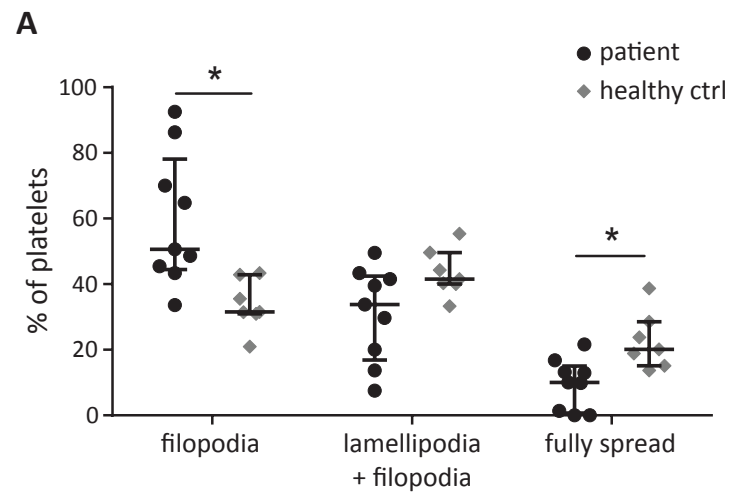

B
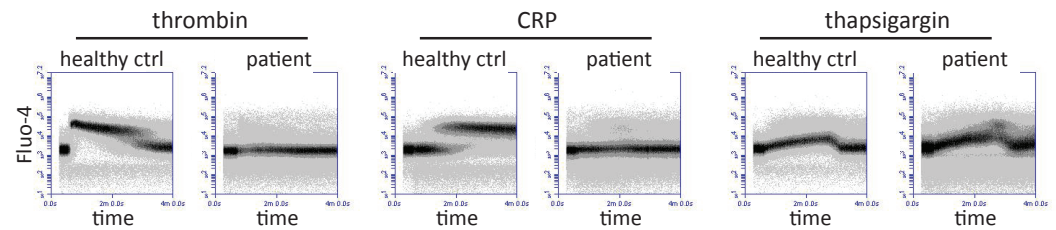

C

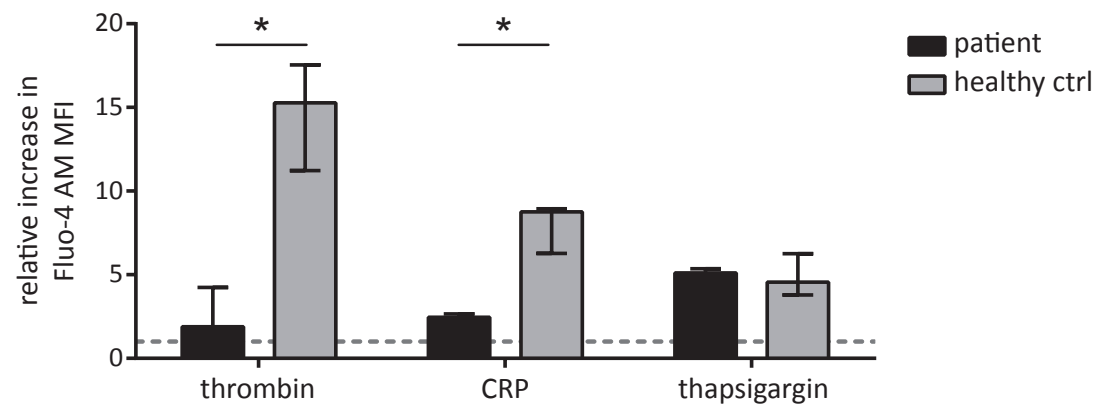

Figure 3: Impaired platelet spreading and $\mathrm{Ca}^{2+}$ signaling of platelets from patients . A) Platelets from patients or healthy controls were allowed to spread on a fibrinogen surface for $10 \mathrm{~min}$, after which microscopic images were captured. Spreading state per platelet was classified in three stages based on morphology: (i) filopodia only, (ii) filopodia and lamellipods, or (iii) fully spread. Percentages of platelets per category are shown. Medians (with IQR) for 9 patients, 7 control subjects. B, C) Fluo-4-loaded platelets from patients $(n=7)$ and controls $(n=5)$ were stimulated with thrombin $(4 \mathrm{nM}), \mathrm{CRP}-\mathrm{XL}(10 \mu \mathrm{g} / \mathrm{mL})$ or thapsigargin $(0.5 \mu \mathrm{M})$ in the presence of $2 \mathrm{mM} \mathrm{CaCl}_{2}$. Changes in Fluo-4 fluorescence were measured in time by flow cytometry. B) Representative Fluo-4 traces in time. C) Relative increases in cytosolic $\mathrm{Ca}^{2+}$. Medians with IQR, ${ }^{*} p<0.05$. Overall platelet responsiveness of the patients was $31.5-57.9 \%$ (IQR). 
of the platelets from control subjects were in stages 2-3, while the patient platelets predominantly stayed in stage 1 (forming filopodia only), with few platelets being fully spread (Figure $3 \mathrm{~A}$ ). This suggested a diminished integrin activity and outside-in signaling in the patient platelets.

We further examined agonist-induced $\mathrm{Ca}^{2+}$ signaling after loading the platelets with Fluo-4. Stimulation with thrombin or CRP-XL induced only a small rise in $\left[\mathrm{Ca}^{2+}\right]_{i}$ in patient platelets, when compared to control platelets (Figure 3B-C). On the other hand, the $\left[\mathrm{Ca}^{2+}\right]$ rise induced by thapsigargin (an inhibitor of endoplasmic reticulum $\mathrm{Ca}^{2+}$-ATPases), as a measure of $\mathrm{Ca}^{2+}$ store content, ${ }^{20}$ was similar for patient and control platelets. Together, this pointed to a defective agonist-induced $\mathrm{Ca}^{2+}$ signaling machinery, independently of receptor type (i.e., PAR1/4 or GPVI receptors).
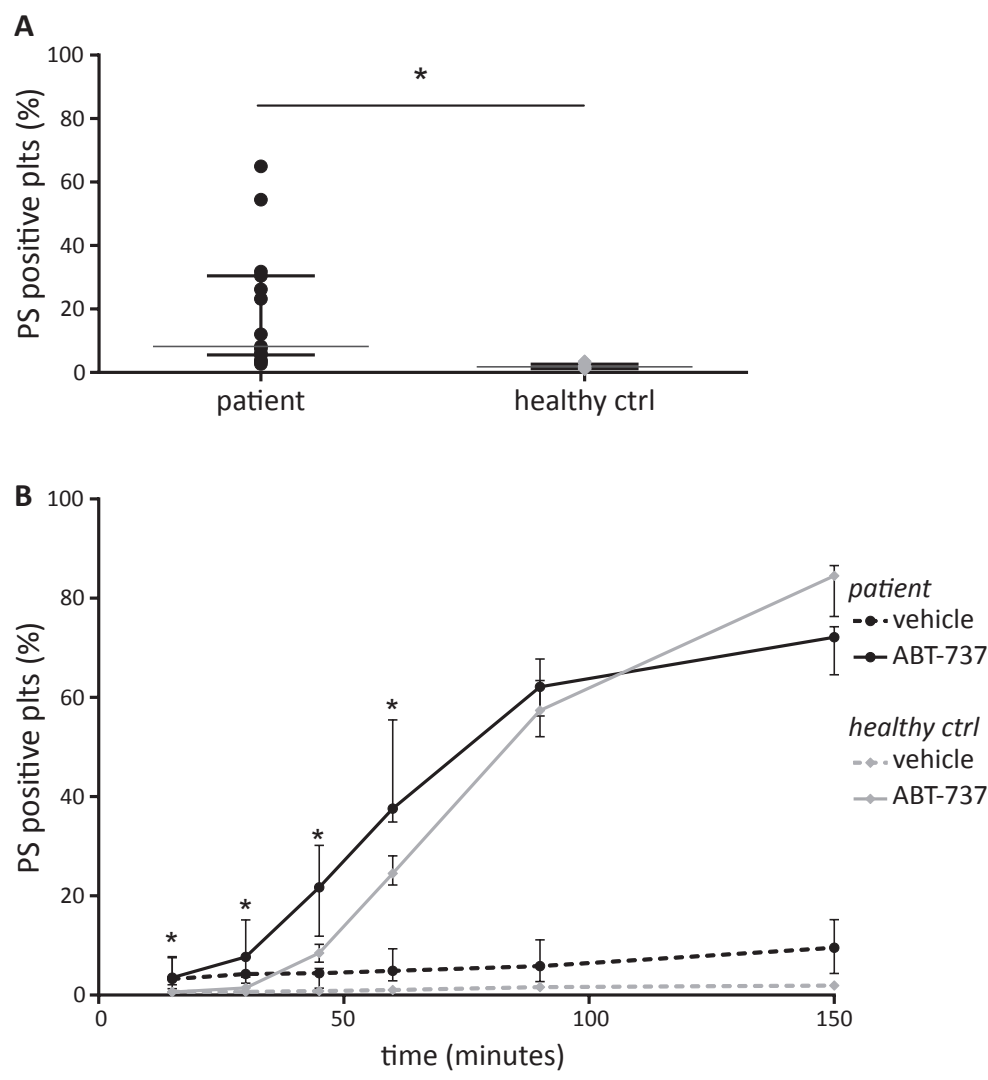

Figure 4: Increased PS exposure in platelets from patients receiving chemotherapy. Isolated platelets from patients and controls were incubated at $37^{\circ} \mathrm{C}$ for $90 \mathrm{~min}$, and stained for PS exposure with FITC-annexin A5. A) Percentages of PS-exposing platelets. Medians with IQR (patients $n=15$, controls $n=12$ ). B) PS exposure measured after indicated times with vehicle or $5 \mu \mathrm{M}$ ABT-737 to induce apoptosis. Medians with IQR ( $n=$ $6-9),{ }^{*} p<0.05$. Overall platelet responsiveness of the patients was $30.1-44.9 \%$ (IQR). 
A

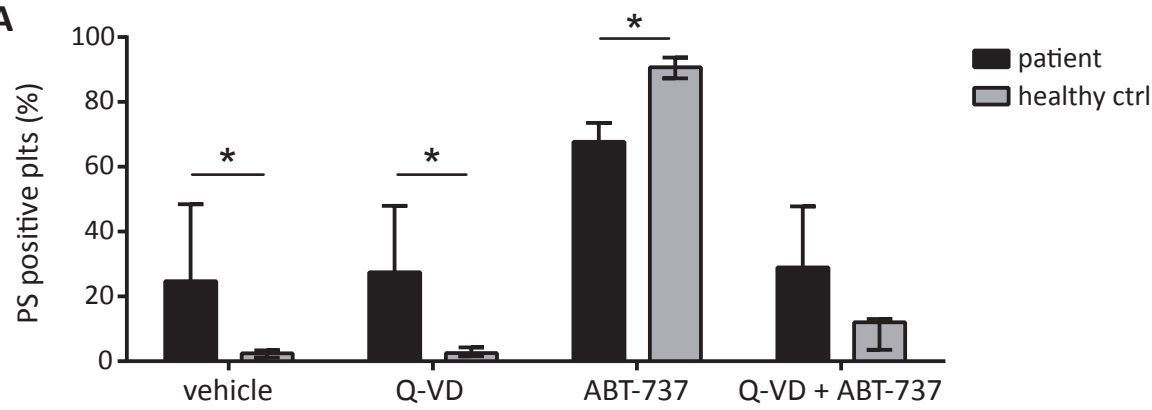

B

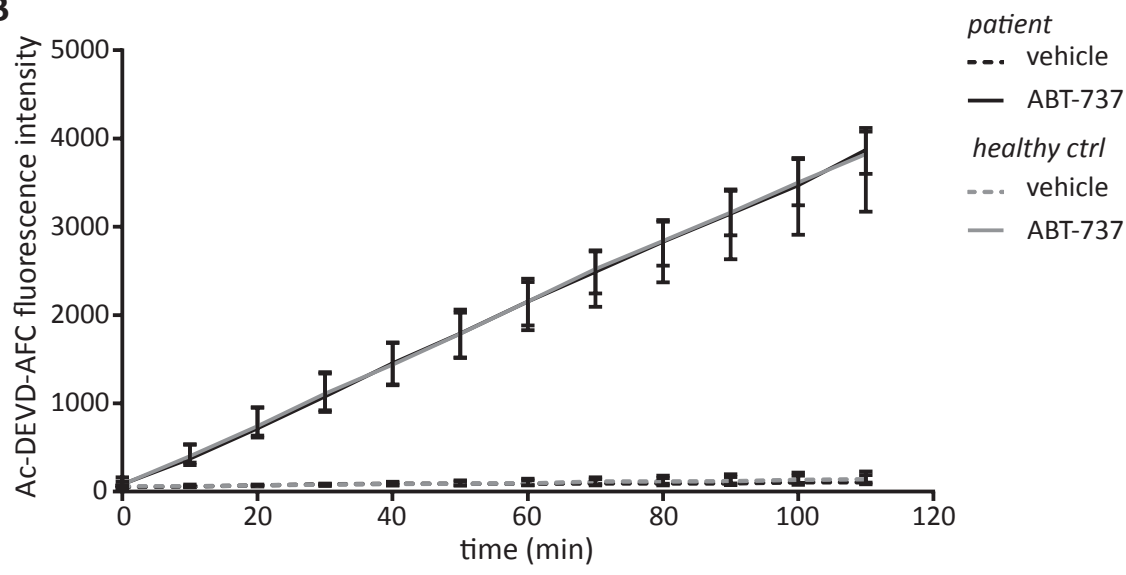

C

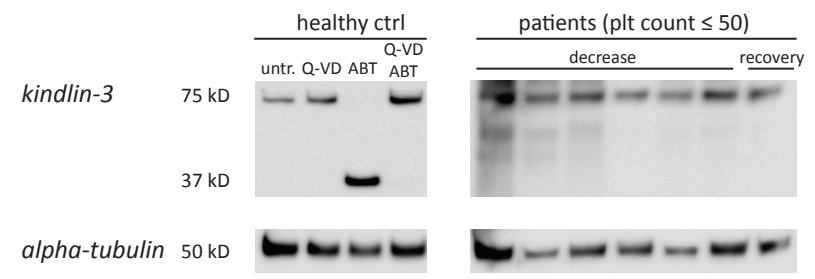

Figure 5: Absence of classical apoptosis in patient platelets. Platelets $\left(10 \times 10^{6} / \mathrm{mL}\right)$ from patients or controls were pretreated with caspase inhibitor Q-VD-OPh $(10 \mu \mathrm{M})$, as indicated, and then stimulated with ABT-737 $(5 \mu \mathrm{M})$ or vehicle. A) Fractions of platelets with PS exposure, measured with FITC-annexin A5. B) Caspase-3 activity determined with a fluorometric assay. C) Absence of caspase-dependent kindlin-3 cleavage in western blots from patient platelets. Control platelets were stimulated with ABT-737 with(out) Q-VD-OPh pretreatment; patient platelets were analyzed during the decreasing and recovery phases of platelet count. Medians with IQR ( $n=4-8),{ }^{*} p<0.05$. Overall platelet responsiveness of the patients was $26.3-50.8 \%$ (IQR).

Impaired mitochondrial bioenergetics but no apoptosis in platelets after chemotherapy

Given the cytotoxicity of chemotherapeutic compounds, we evaluated if patient platelets showed characteristics of apoptosis, since this process is known to lead to dysfunctional signaling. ${ }^{21}$ As a marker of apoptosis, PS exposure was determined by FITC-annexin A5 binding. In contrast to control platelets, the patient platelets were prone to expose PS upon short-term storage without external stimuli (Figure 4A). Upon 
stimulation with the BH3 mimetic ABT-737, triggering the intrinsic pathway of apoptosis, ${ }^{21}$ PS exposure was initially accelerated in the patient platelets, when compared to control platelets (Figure 4B). As expected, preincubation with the pan-caspase inhibitor Q-VDOPh fully inhibited the PS exposure triggered by ABT-737. However, Q-VD-OPh failed to affect the storage-dependent PS exposure (Figure 5A). Furthermore, whereas ABT-737 stimulation resulted in high caspase-3 activity, no such activity could be detected during storage (Figure 5B). Additional confirmation for the absence of apoptotic signaling was
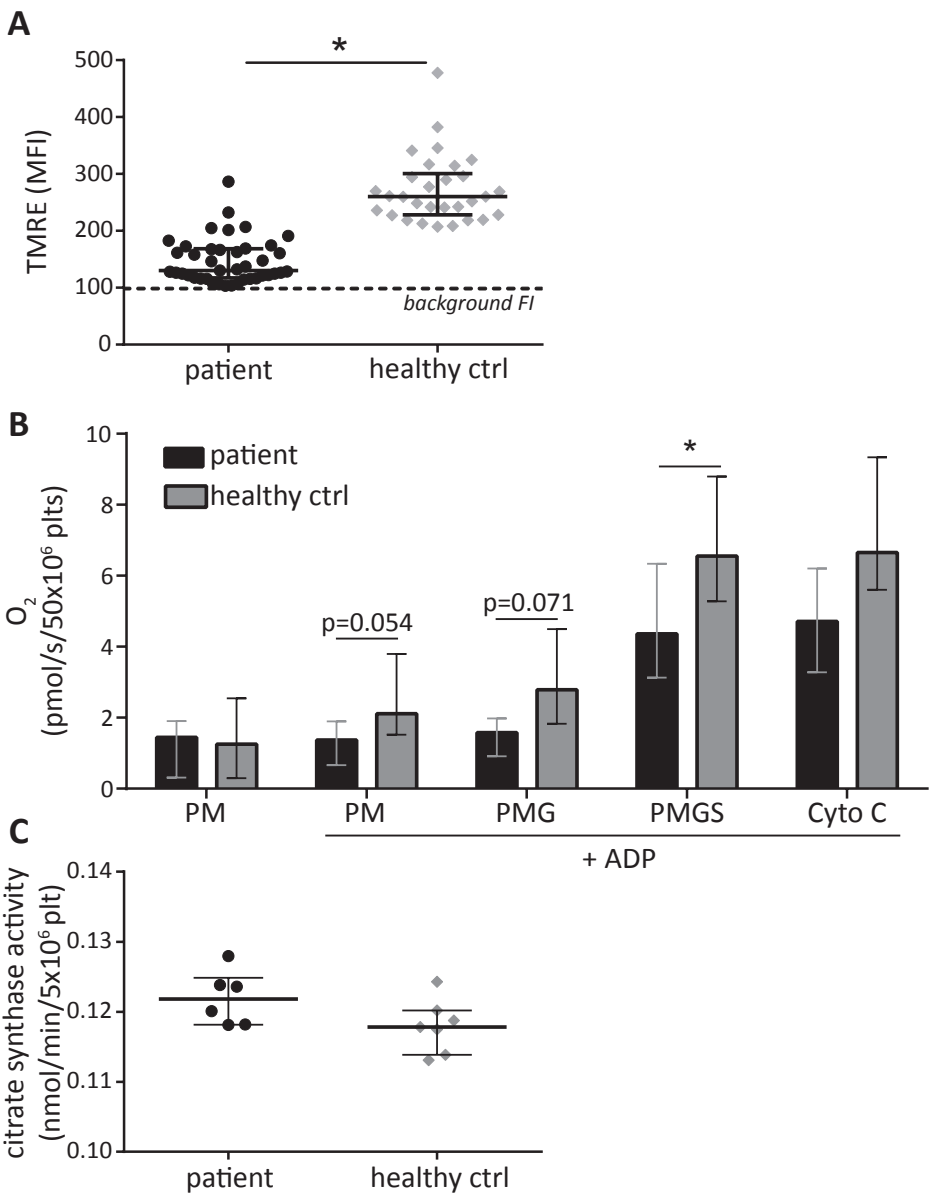

Figure 6: Impaired mitochondrial bioenergetics in patient platelets. A) Initial screening of TMRE staining of washed platelets from patients. To assess the mitochondrial membrane potential, platelets were stained with TMRE and subsequently, analyzed by flow cytometry. Shown are mean fluorescence intensities of TMRE. ( $n=39$ : treatment classes: A+B: $n=10 ; \mathrm{A}+\mathrm{B}+\mathrm{C}: n=5 ; \mathrm{B}+\mathrm{C}: n=7 ; \mathrm{C}: n=13)$ and healthy controls $(n=27)$. B) High resolution respirometry to measure mitochondrial respiration in washed platelets from additionally included patients $(n=7)$ and controls $(n=9)$. Depicted is oxygen consumption due to sequential addition of saturating amounts of pyruvate (P), malate (M), ADP, glutamate (G), succinate $(S)$ and cytochrome $C(C y$ to $C)$. C) Citrate synthase activity in washed platelets from patients $(n=6)$ and controls $(n=7)$ to assess mitochondrial content. Medians with IQR, ${ }^{*} p<0.05$. Overall platelet responsiveness of the patients was $28.7-46.9 \%$ (IQR). 
obtained by assessing the caspase-dependent cleavage of the integrin-binding protein, kindlin-3. ${ }^{22}$ Western blot analysis indicated that, in platelets from control subjects, ABT737 treatment induced full cleavage of kindlin-3, which was prevented by Q-VD-OPh (Figure 5C). In the patient platelets (with confirmed functional impairment of integrin activation and P-selectin expression), however, no kindlin-3 cleavage could be detected in the absence of ABT-737.

Platelet activation is known to rely on mitochondrial activity for sufficient ATP production. ${ }^{23}$ Given that mitochondrial impairment can lead to PS exposure, ${ }^{24,25}$ we assessed the activity of mitochondria in several ways. As part of the initial characterization of the patient platelets, the mitochondrial membrane potential was assessed by staining with TMRE. Whereas control platelets displayed high TMRE staining, the patient platelets showed much less staining intensity (Figure 6A). This suggested a depolarization of the platelet mitochondria, which was independent of diagnosis or treatment class, KruskalWallis $\mathrm{H}$ test, $p=0.656$ and $p=0.126$, respectively). Markedly, the low TMRE fluorescence correlated well to the reduced platelet responsiveness (Spearman's rho $=0.569, p=0.001$ ).

Given these results with TMRE staining, we subsequently decided to assess mitochondrial activity in platelet directly, by measuring the mitochondrial respiration using high-resolution respirometry. ${ }^{26}$ In the presence of saturating amounts of complex I and II substrates of the oxidative phosphorylation (OXPHOS) chain, i.e. pyruvate, malate, ADP, glutamate and succinate, we found that the maximal ADP-supported respiration of mitochondria was significantly lower in platelets from patients than in control cells (Figure $6 \mathrm{~B})$. To check whether the mitochondrial content was altered as well, we measured citrate synthase activity. ${ }^{27}$ Here, no difference was found between the platelets from patients and healthy controls (Figure 6C). Therefore, the reduced TMRE fluorescence and the diminished mitochondrial respiration did not seem to be due to a lower mitochondrial content. Transmission electron microscopic images were recorded from patient platelets, and these did not reveal structural abnormalities of the mitochondria (data not shown).

\section{Improved overall platelet function by transfusion}

For 36 of the patients, blood samples could be obtained before and $1 \mathrm{~h}$ after platelet transfusion. In the dataset, all major treatment groups were represented. As expected, platelet count increased after transfusion (Table S3). The clinical efficacy of transfusion was evaluated from the corrected count increment $[\mathrm{CCl}$ : (platelet count increment $\mathrm{x}$ body surface area)/(number of transfused platelets $\left.\left.\times 10^{11}\right)\right] .{ }^{28}$ This was adequate for $96 \%$ of the patients, as indicated by a CCI value of $>7.5$ (median: 14.8, IQR: 11.3-18.0).

Flow cytometric analysis of integrin activation and P-selectin expression demonstrated that at $1 \mathrm{~h}$ after transfusion, platelet responsiveness was improved for most patients (Figure 7). Whenever possible, platelets were also isolated from the remainder of the transfusion concentrates. It appeared that the activity of the circulating platelets after transfusion approached that of the platelets of the concentrates, when triggered with thrombin or CRP-XL. However, the responsiveness to ADP of the circulating platelets after transfusion was higher than in concentrates (integrin $\alpha_{11 b} \beta_{3}$ activation, $p=0.002$ ). In the post-transfusion samples, TMRE fluorescence intensity was also increased (126 vs. 192, $p=0.001$ ), but it was still lower than in the platelet concentrates (192 vs. 274, 
A

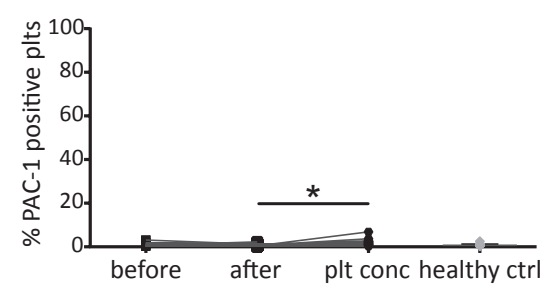

\section{B}

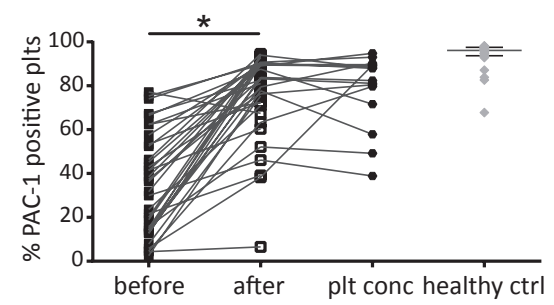

C

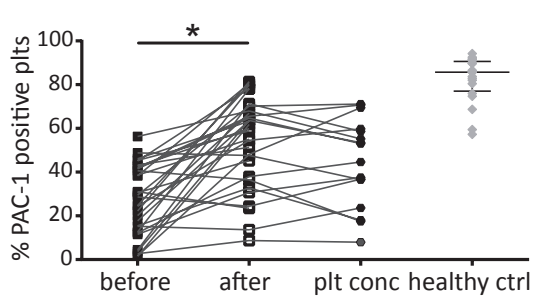

D

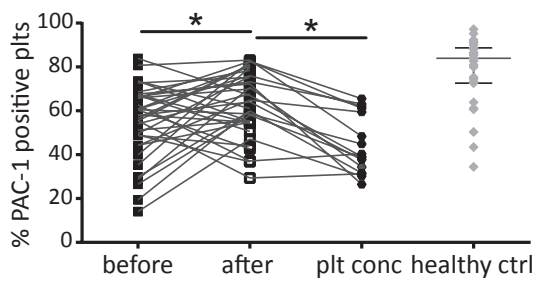

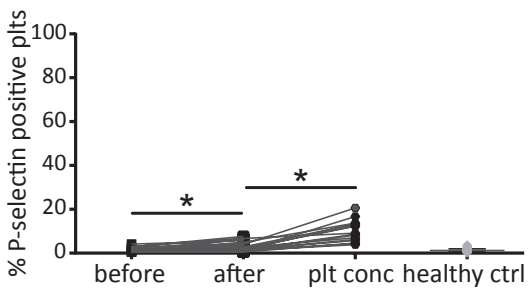
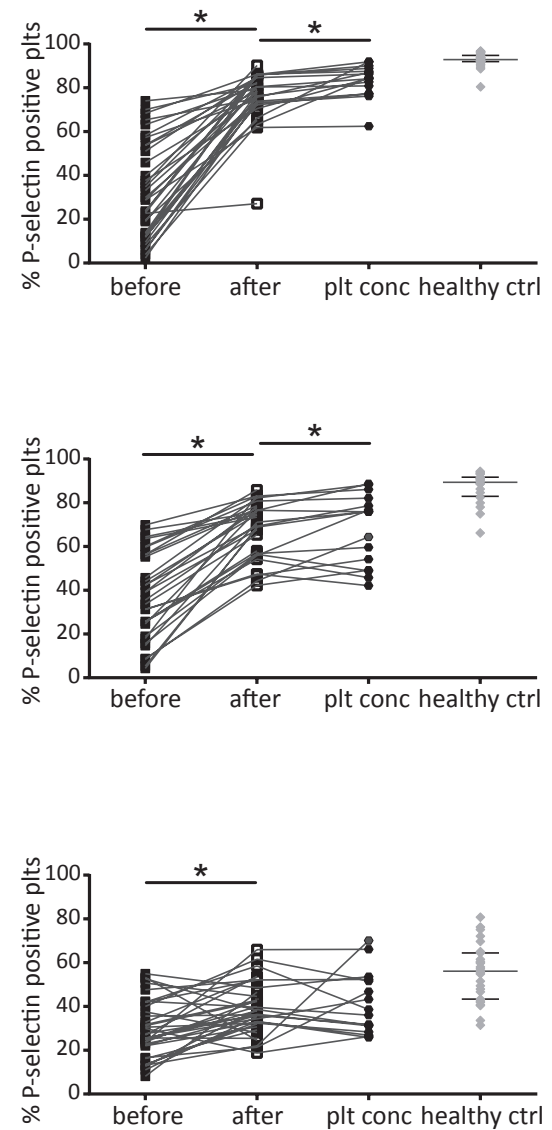

Figure 7: Transfusion partly normalizes platelet responsiveness in chemotherapy treated patients with thrombocytopenia. Washed platelets $\left(10 \times 10^{6} / \mathrm{mL}\right)$ analyzed from healthy controls, patients with chemotherapy-induced thrombocytopenia before and after transfusion, and from the transfused platelet concentrates. Integrin $\alpha_{11 b} \beta_{3}$ activation and P-selectin expression were measured in resting platelets (A), and after stimulation with thrombin (B), CRP-XL (C) or 2MeS-ADP (D). Median values with IQR for patients $(n=36$ : treatment classes: $\mathrm{A}+\mathrm{B}: n=7 ; \mathrm{A}+\mathrm{B}+\mathrm{C}: n=10 ; \mathrm{B}+\mathrm{C}: n=4 ; \mathrm{C}: n=11)$ and platelet concentrates $(n=16)$ and control subjects $(n=27) ;{ }^{*} p<0.05$. 
$p=0.01)$. The overall platelet responsiveness showed a better correlation with platelet count after transfusion (Spearman's $r h o=0.494, p=0.003$ ) than before transfusion.

\section{Similar coagulation and reduced thrombus formation after chemotherapy}

Standard coagulation parameters could be determined in plasmas from 22 patients after the chemotherapy treatment. For $70 \%$ of the patients, values of aPTT, PT or thrombin time were within reference range (Table S4). Fibrinogen and VWF levels were slightly elevated, while D-dimer levels were substantially increased in the patient plasmas. On the other hand, factor VII activity levels were decreased.

To determine the overall pro-hemostatic effect of the platelet transfusion, thrombus and fibrin formation under flow were measured. Therefore, whole blood was perfused under recalcification over a collagen and a collagen/tissue factor surface. ${ }^{29}$ In pretransfusion blood samples (platelet count $\leq 10 \times 10^{6} / \mathrm{mL}$ ), only few platelets adhered and small aggregates were formed with little fibrin on both the collagen (platelet deposition $0.96 \%$, fibrin area coverage $0.14 \%$ ) and the collagen/tissue factor surface (platelet deposition $1.51 \%$, fibrin area coverage $0.77 \%$ ), when compared to previously established normal values (Figure S2). ${ }^{29}$ After transfusion, platelet and fibrin deposition significantly increased $(p<0.01)$, which was accompanied by a larger aggregate size $(p<0.01)$ (Figure S2). Taken together, these results confirm the acquired platelet defect, and indicate that the improvement in overall platelet function after transfusion ameliorates hemostasis.

\section{Discussion}

In this paper, we provide novel evidence that the platelets from thrombocytopenic patients, suffering from hematological malignancies and treated with myeloablative chemotherapy, are dysfunctional in multiple aspects. We found that key agonist-induced responses of the patients' platelets, such as integrin activation, secretion and $\mathrm{Ca}^{2+}$ fluxes are impaired, remarkably at a variable extent. Furthermore, the platelets from almost all patients showed agonist-independent exposure of PS upon storage, which was not linked to apoptotic caspase activity; such in contrast to the platelets from healthy subjects, not displaying PS exposure. In the patients' platelets, the defective activation could be linked to an impaired mitochondrial membrane potential and a decreased mitochondrial respiratory activity. Transfusion of the patients with platelet concentrate improved the hemostatic potential by enhancing thrombus and fibrin formation.

The impaired platelet responsiveness after myeloablative chemotherapy (median of 8 days) was only weakly correlated to the whole blood platelet count, indicating that the extent of thrombocytopenia as such was not a main factor in the dysfunction. In agreement with this conclusion, in patients with a recovering platelet count after transplantation, the functionality of the platelets improved towards normal. Detailed analysis indicated that neither disease type nor chemotherapy regimen could explain the inter-patient variation in platelet responsiveness. This points to other factors determining the severity of dysfunction, such as a different sensitivity of megakaryocytes in the bone marrow to the previous chemotherapy treatment. As the sensitivity of megakaryocytic 
precursor cells to chemotherapeutics is known to vary, ${ }^{30}$ the extent of platelet dysfunction might be a combined result of the sensitivity of the precise drugs administered and their dosing.

The dysfunction of platelets identified in this patient group markedly differs from the so-called 'exhausted' platelets, which have been described for patients with solid tumors. ${ }^{31}$ Exhausted platelets were characterized by a high integrin activation and P-selectin expression in the absence of stimulating agents, and a reduced increase in the parameters after agonist stimulation. These changes might point to platelet activation in vivo, resulting in a secondary loss of function..$^{32}$ Given that in the present patient group P-selectin expression and integrin activation were low without stimulation, there is no evidence for in vivo platelet activation linked to chemotherapy treatment. On the other hand, the patients' platelets showed an tendency to expose PS, which is compatible with an apoptotic process, as apoptotic platelets are known to be defective in aggregation and secretion. ${ }^{21}$ However, ongoing apoptotic signaling could be excluded, since: (i) treatment with the pan-caspase inhibitor Q-VD-OPh did not prevent PS exposure, (ii) measurable caspase-3 activity was absent, and (iii) caspase-dependent cleavage of kindlin-3 could not be detected.

Platelets rely on mitochondrial ATP production, in particular upon activation when their energy demand increases. ${ }^{23}$ While the mitochondrial content and ultrastructure appeared normal in the patients' platelets, we noticed a marked reduction of the platelet mitochondrial membrane potential and the mitochondrial oxidative phosphorylation. Other authors have shown that anti-tumor antibiotics (anthracyclines), an important class of chemotherapeutic agents used to treat hematological malignancies, induce cardiotoxicity and muscle weakness due to impairment of mitochondrial function via increased production of reactive oxygen species (ROS). ${ }^{33-35}$ In cardiac cells, the accumulation of iron inside the mitochondria may contribute to the production of ROS. ${ }^{36}$ Furthermore, the mitochondrial activity in myocardial and hepatic cells is known to be impaired by the chemotherapeutics cyclophosphamide and carmustine (BCNU). ${ }^{37-39}$ This suggests that, also in megakaryocytes and platelets, a similar mechanism of ROS-linked mitochondrial dysfunction is operative. Our findings together imply that a dysfunction in the mitochondria impairs platelet activity and induces PS exposure upon storage, thus leading to a shorter platelet life time. This phenomenon, next to the previous drug effects on (pro-)platelet production, thus may explain the rapid decline in platelet number and function. This hypothesis is supported by a recent study in mice, developing thrombocytopenia after 5-fluororacil treatment. In these animals low-level laser therapy was found to increase the mitochondrial activity of megakaryocytes, resulting in a normalization of hemostasis. ${ }^{40}$

Due to ethical limitations, we could not assess whether the platelet dysfunction after chemotherapeutic treatment was linked to abnormal (pro)platelet formation from megakaryocytes in the bone marrow. The available literature yet suggests that the progenitor cells are more vulnerable towards chemotherapy than matured megakaryocytes. ${ }^{30}$ In patients who received chemotherapy and had not yet developed 
thrombocytopenia, we observed a normal platelet activity. This agrees with an indirect drug effect on the megakaryocytes or precursor cells, rather than a direct effect on the circulating platelets.

With regards to the coagulant state, the reduced level of factor VII found in combination with high circulating D-dimers in the patients' plasmas is suggestive for an mild ongoing state of tissue factor-triggered coagulation. ${ }^{41}$ However, the data do not provide evidence for appreciable consumption of other coagulation factors. Given that factor VII has a short half-life in blood, ${ }^{42}$ it will be the first coagulation factor to decline upon ongoing coagulation. Chemotherapy can induce endothelial cell activation and upregulate tissue factor levels ${ }^{43}$ which also can explain the elevated VWF levels in patients. Interestingly, we found improved overall platelet responsiveness after transfusion with platelet concentrates in the majority of patients. In addition, platelet thrombus formation and fibrin formation were significantly ameliorated after transfusion.

The present study has several limitations. Given that the number of isolated platelets was limited due to the severe thrombocytopenia, only a restricted subset of measurements could be performed per patient blood sample, with as a consequence that different patient samples needed to be used for some of the measurements. Furthermore, platelet samples were analyzed from patients with different disease types (AML/ALL, multiple myeloma and malignant lymphoma) after receiving chemotherapy in distinct treatment regimens. Here we like to stress that a reduced platelet function was detected in all patient groups and all therapeutic regimens.

Current guidelines for prophylactic transfusion during myelosuppression are based on platelet count only. Our novel findings indicate that, next to the platelet count, also the activity of circulating platelets needs to be considered for an optimal control of hemostasis. This work thus urges for an inclusion of platelet function assays for the prediction of bleeding in this patient group.

\section{Key points}

- Platelets from patients who are treated with chemotherapy and have thrombocytopenia are functionally impaired.

- The platelet dysfunction is linked to impaired mitochondrial activity, distinct from ongoing apoptosis. 


\section{Acknowledgements}

We thank all medical students involved for assisting in patient inclusion.

\section{Sources of funding}

Funding for this project was provided by the EHA-ISTH Research Fellowship granted by the European Hematology Association and the International Society of Thrombosis and Haemostasis to PvdM.

\section{Authorship and disclosures}

Contribution: $\mathrm{CB}$ performed experiments, analyzed data and wrote the paper; FS, RW, RO, HH, GH performed experiments and analyzed data; $\mathrm{FM}$ and EB recruited the patients; $\mathrm{YH}$ contributed analytical support; HtC drafted the paper; JH and PvdM designed and coordinated the research and wrote the paper. All authors have seen and approved the final manuscript.

Conflict of interest: The authors declare no relevant conflicts of interest.

Correspondence: Paola E.J. van der Meijden, PhD, Dept. of Biochemistry (CARIM), Maastricht University, P.O. Box 616, 6200 MD Maastricht, the Netherlands. Tel: +31-433881684, fax: +31-43-3884159; e-mail: p.vandermeijden@maastrichtuniversity.nl 


\section{References}

1. Versteeg $\mathrm{HH}$, Heemskerk JW, Levi M, Reitsma PH. New fundamentals in hemostasis. Physiol Rev. 2013;93:327-58.

2. Apelseth TO, Hervig T, Bruserud $\varnothing$. Current practice and future directions for optimization of platelet transfusions in patients with severe therapy-induced cytopenia. Blood Rev. 2011;25:113-22.

3. Estcourt LJ, Stanworth SJ, Doree C, Hopewell S, Murphy MF, Tinmouth A, et al. Prophylactic platelet transfusion for prevention of bleeding in patients with haematological disorders after chemotherapy and stem cell transplantation. Cochrane Database Syst Rev. 2012;16(5):CD004269.

4. Wandt H, Schaefer-Eckart K, Wendeling K, Pilz B, Wilhelm M, Thalheimer M, et al. Therapeutic platelet transfusion versus routine prophylactic transfusion in patients with haematological malignancies: an openlabel, multicentre, randomised study. Lancet. 2012;380(9850):1309-16.

5. Stanworth SJ, Estcourt LJ, Powter G, Kahan BC, Dyer C, Choo L, et al. A no-prophylaxis platelet-transfusion strategy for hematologic cancers. N Engl J Med. 2013;368:1771-80.

6. Friedmann AM, Sengul H, Lehmann H, Schwartz C, Goodman S. Do basic laboratory tests or clinical observations predict bleeding in thrombocytopenic oncology patients? A reevaluation of prophylactic platelet transfusions. Transfus Med Rev. 2002;16(1):34-45.

7. Slichter SJ. Relationship between platelet count and bleeding risk in thrombocytopenic patients. Transfus Med Rev. 2004;18(3):153-67.

8. Cowan DH, Graham RC, Baunach D. The platelet defect in leukemia. J Clin Invest. 1975;56:188-200.

9. Woodcock BE, Cooper PC, Brown PR, Pickering C, Winfield DA, Preston FE. The platelet defect in acute myeloid leukaemia. J Clin Pathol. 1984;37:1339-42.

10. Leinoe EB, Hoffmann MH, Kjaersgaard E, Johnsen HE. Multiple platelet defects identified by flow cytometry at diagnosis in acute myeloid leukaemia. Br J Haematol. 2004;127:76-84.

11. Leinoe EB, Hoffmann MH, Kjaersgaard E, Nielsen JD, Bergmann OJ, Klausen TW, et al. Prediction of haemorrhage in the early stage of acute myeloid leukemia by flow cytometric analysis of platelet function $\mathrm{Br}$ J Haematol. 2005;128(4):526-32.

12. Kuter DJ. Managing thrombocytopenia associated with cancer chemotherapy. Oncology (Williston Park). 2015;29(4):282-94.

13. Pogliani EM, Fantasia R, Lambertenghi-Deliliers G, Cofrancesco E. Daunorubicin and platelet function. Thromb Haemost. 1981;45(1):38-42.

14. Lanzi C, Banfi P, Ravagnani F, Gambetta RA. Diversity of effects of two antitumor anthracycline analogs on the pathway of activation of PKC in intact human platelets. Biochem Pharmacol. 1988;37(18):3497-504.

15. Foss B, Ulvestad E, Hervig T, Bruserud $\varnothing$. Effects of cytarabine and various anthracyclins on platelet activation: characterization of in vitro effects and their possible clinical relevance in acute myelogenous leukemia. Int J Cancer. 2002;97:106-14.

16. Moreau P, San Miguel J, Sonneveld P, Mateos MV, Zamagni E, Avet-Loiseau H, et al. Multiple myeloma: ESMO clinical practice guidelines for diagnosis, treatment and follow-up. Ann Oncol. 2017; Epub ahead of print.

17. HOVON - the Haemato Oncology Foundation for Adults in the Netherlands. Trials AML 2017. Available from: http://www.hovon.nl/trials/trials-by-type/aml.html.

18. HOVON - the Haemato Oncology Foundation for Adults in the Netherlands. Trials NHL 2017. Available from: http://www.hovon.nl/trials/trials-by-type/nhl.html.

19. American Cancer Society. How chemotherapy drugs work 2016 [updated February 11, 2016]. Available from: https://www.cancer.org/treatment/treatments-and-side-effects/treatment-types/chemotherapy/ how-chemotherapy-drugs-work.html.

20. Smeets EF, Heemskerk JW, Comfurius P, Bevers EM, Zwaal RF. Thapsigargin amplifies the platelet procoagulant response caused by thrombin. Thromb Haemost. 1993;70(6):1024-9.

21. Vogler M, Hamali HA, Sun XM, Bampton ETW, Dinsdale D, Snowden RT, et al. BCL2/BCL-X inhibition induces apoptosis, disrupts cellular calcium homeostasis, and prevents platelets activation. Blood. 2011;117:7145-54.

22. Solari FA, Mattheij NJ, Burkhart JM, Swieringa F, Collins PW, Cosemans JM, et al. Combined quantification of the global proteome, phosphoproteome, and proteolytic cleavage to characterize altered platelet functions in the human Scott syndrome. Mol Cell Proteomics. 2016;15(10):3154-69. 
23. Kramer PA, Ravi S, Chacko B, Johnson MS, Darley-Usmar VM. A review of the mitochondrial and glycolytic metabolism in human platelets and leukocytes: Implications for their use as bioenergetic biomarkers. Redox Biol. 2014;2:206-10.

24. Mattheij NJ, Gilio K, van Kruchten R, Jobe SM, Wieschhaus AJ, Chishti AH, et al. Dual mechanism of integrin aiibb3 closure in procoagulant platelets. J Biol Chem. 2013;288(19):13325-36.

25. van Kruchten R, Mattheij NJA, Saunders C, Feijge MA, Swieringa F, Wolfs JLN, et al. Both TMEM16Fdependent and TMEM16F-independent pathways contribute to phosphatidylserine exposure in platelet apoptosis and platelet activation. Blood. 2013;121(10):1850-7.

26. Lanza IR, Nair KS. Mitochondrial metabolic function assessed in vivo and in vitro. Curr Opin Clin Nutr Metab Care. 2010;13(5):511-7.

27. Larsen S, Nielsen J, Hansen CN, Nielsen LB, Wibrand F, Stride N, et al. Biomarkers of mitochondrial content in skeletal muscle of healthy young human subjects. J Physiol. 2012;590(14):3349-60.

28. CBO. Blood transfusion guidelines- the Netherlands. 2011.

29. Swieringa F, Baaten CC, Verdoold R, Mastenbroek TG, Rijnveld N, van der Laan KO, et al. Platelet control of fibrin distribution and microelasticity in thrombus formation under flow. Arterioscler Thromb Vasc Biol. 2016;36:692-9.

30. Zeuner A, Signore M, Martinetti D, Barturcci M, Peschle C, De Maria R. Chemotherapy-induced thrombocytopenia derives from the selective death of megakaryocyte progenitors and can be rescued by stem cell factor. Cancer Res. 2007;67(10):4767-73.

31. Riedl J, Kaider A, Marosi C, Prager GW, Eichelberger B, Assinger A, et al. Decreased platelet reactivity in patients with cancer is associated with high risk of venous thromboembolism and poor prognosis. Thromb Haemost. 2017;117.

32. Baaten CC, Ten Cate H, van der Meijden PE, Heemskerk JW. Platelet populations and priming in hematological diseases. Blood Rev. 2017; Epub ahead of print.

33. Gilliam LAA, Fisher-Wellman KH, Lin CT, Maples JM, Cathey BL, Neufer PD. The anticancer agent doxorubicin disrupts mitochondrial energy metabolism and redox balance in skeletal muscle. Free Radic Biol Med. 2013;65:988-96.

34. Sorensen JC, Cheregi BD, Timpani CA, Nurgali K, Hayes A, Rybalka E. Mitochondria: Inadvertent targets in chemotherapy-induced skeletal muscle toxicity and wasting? Cancer Chemother Pharmacol. 2016;78(4):67383.

35. Gouspillou G, Scheede-Bergdahl C, Spendiff S, Vuda M, Meehan B, Mlynarski H, et al. Anthracyclinecontaining chemotherapy causes long-term impairment of mitochondrial respiration and increased reactive oxygen species release in skeletal muscle. Sci Rep. 2015;5:8717.

36. Ichikawa Y, Ghanefar M, Bayeva M, Wu R, Khechaduri A, Naga Prasad SV, et al. Cardiotoxicity of doxorubicin is mediated through mitochondrial iron accumulation. J Clin Invest. 2014;124(2):617-30.

37. al-Nasser IA. In vivo prevention of cyclophosphamide-induced $\mathrm{Ca}^{2+}$ dependent damage of rat heart and liver mitochondria by cyclosporin A. Comp Biochem Physiol A Mol Integr Physiol. 1998;121(3):209-14.

38. Prasad SB, Rosangkima G, Nicol BM. Cyclophosphamide and ascorbic acid-mediated ultrastructural and biochemical changes in Dalton's lymphoma cells in vivo. Eur J Pharmacol. 2010;645(1-3):47-54.

39. Kang PT, Chen CL, Ren P, Guarini G, Chen YR. BCNU-induced gR2 defect mediates S-glutathionylation of complex I and respiratory uncoupling in myocardium. Biochem Pharmacol. 2014;89(4):490-502.

40. Zhang Q, Dong T, Li P, Wu MX. Noninvasive low-level laser therapy for thrombocytopenia. Sci Transl Med. 2016;8(349):349ra101.

41. Mackman N, Tilley RE, Key NS. Role of the extrinsic pathway of blood coagulation in hemostasis and thrombosis. Arterioscler Thromb Vasc Biol. 2007;27(8):1687-93.

42. Hoffbrand AV, Moss PAH. Essential Haematology. $6^{\text {th }}$ ed: Wiley-Blackwell; 2011.

43. Giordano P, Molinari AC, Del Vecchio GC, Saracco P, Russo G, Altomare M, et al. Prospective study of hemostatic alterations in children with acute lymphoblastic leukemia. Am J Hematol. 2010;85(5):325-30.

44. Briggs C, Kunka S, Hart D, Oguni S, Machin SJ. Assessment of an immature platelet fraction (IPF) in peripheral thrombocytopenia. Br J Haematol. 2004;126(1):93-9. 


\section{Supplemental Methods}

\section{Materials}

Apyrase, bovine serum albumin (BSA), calcium chloride, the citrate synthase activity kit, dimethylsulfoxide, glucose, magnesium chloride and thrombin were obtained from Sigma (St. Louis, MO, USA). Collagen related peptide (CRP) was purchased from Dr. R. Farndale (Cambridge, UK). ABT-737, 2MeS-ADP and thapsigargin were from SantaCruz Biotechnology (Santa Cruz, CA, USA); fluorescein isothiocyanate (FITC)-conjugated PAC1 antibody against active integrin $\alpha_{\| b} \beta_{3}$ and APC-conjugated anti-CD61 antibody from Becton-Dickinson Bioscience (Franklin Lakes, NJ, USA). Alexa Fluor 546 conjugated human fibrinogen; Fluo-4 AM and NuPAGE LDS sample buffer NOVEX were from Invitrogen Life Technologies (Bleiswijk, The Netherlands). FITC-conjugated anti-P-selectin antibody came from Beckman Coulter, rabbit anti- $\alpha$-tubulin antibody from Abcam (Cambridge, UK) and annexin-A5 FITC-conjugated was from PharmaTarget (Maastricht, The Netherlands). Collagen type I came from Nycomed Pharma (Munich, Germany); innovin (tissue factor) was from Siemens (Erlangen, Germany). Tetramethyl rhodamine methyl ester (TMRE) was from Anaspec (San Jose, CA, USA); Q-VD-Oph from Calbiochem (San Diego, CA, USA) and the fluorometric caspase-3 activity assay from R\&D Systems (Minneapolis, MN, USA). Prestained SDS-PAGE standards were from BioRad (Hercules, CA, USA) and SuperSignal West pico chemiluminescent substrate came from Thermo Scientific (Waltham, MA, USA). Actin FSL Activated PTT reagent, Thrombin reagent and Innovance D-dimer test were from Siemens Healthcare Diagnostics (Den Haag, The Netherlands). Antibody against kindlin-3 was purchased at Cell Signaling Technology (Danvers, MA, USA).

\section{Preparation of washed platelets and plasma}

Platelet isolation procedures were adapted for measurements with low platelet numbers (median $0.36 \times 10^{8}$ platelets per blood sample). Platelet-rich plasma (PRP) was obtained from citrated blood by centrifugation at $290 \mathrm{~g}$ for $2 \mathrm{~min}$. For preparation of washed platelets, PRP in eppendorf tubes was supplemented with 1:10 v/v acidic citrate dextrose (ACD, $80 \mathrm{mM}$ trisodium citrate, $52 \mathrm{mM}$ citric acid and $180 \mathrm{mM}$ glucose) and centrifuged at $2230 \mathrm{~g}$ for $2 \mathrm{~min} .{ }^{1}$ Pelleted platelets were resuspended in Hepes buffer pH 6.6 (10 mM Hepes, $136 \mathrm{mM} \mathrm{NaCl}, 2.7 \mathrm{mM} \mathrm{KCl}, 2 \mathrm{mM} \mathrm{MgCl}, 0.1 \%$ glucose and $0.1 \%$ bovine serum albumin (BSA). After another centrifugation step (2230 g for $2 \mathrm{~min}$ ) in the presence of $1: 15 \mathrm{ACD}+1 \mathrm{U} / \mathrm{mL}$ apyrase, pelleted platelets were resuspended in Hepes buffer pH 7.45 (10 mM Hepes, $136 \mathrm{mM} \mathrm{NaCl}, 2.7 \mathrm{mM} \mathrm{KCl}, 2 \mathrm{mM} \mathrm{MgCl}, 0.1 \%$ glucose and $0.1 \%$ BSA). Platelet count was determined with a Sysmex XP300/XN9000 thrombocounter (Kobe, Japan). Platelet-free plasma was prepared, as described before. ${ }^{1}$

\section{Whole blood cell count and coagulation tests}

Whole blood cell count in $\mathrm{K}_{2}$-EDTA anticoagulated blood was determined using a Sysmex XN-9000 analyzer. Platelet-free plasma was analyzed using a Sysmex CS-2100i analyzer and Dade reagents for: activated partial thromboplastin time (aPTT, Actin FSL), prothrombin time (PT, Innovin), thrombin time (Thrombin reagent), fibrinogen, D-dimers 
(Innovance) and von Willebrand factor antigen. Coagulation factor activities were determined, as described previously. ${ }^{2,3}$ Normal pooled plasma from healthy controls was used as a reference.

\section{Flow cytometric analysis of platelet activation}

To measure platelet activation processes, washed platelets from patients and control subjects (normalized to $10 \times 10^{6}$ platelets $/ \mathrm{mL}$ ) were activated with thrombin (4 $\mathrm{nM}), \mathrm{CRP}-\mathrm{XL}(10 \mu \mathrm{g} / \mathrm{mL})$ or $2 \mathrm{MeS}-\mathrm{ADP}(1 \mu \mathrm{M})$ in the presence of $2 \mathrm{mM} \mathrm{CaCl}$ for $15 \mathrm{~min}$. Integrin $\alpha_{\| b} \beta_{3}$ activation and P-selectin expression were assessed, following labeling with FITC-conjugated PAC-1 antibody $(1.25 \mu \mathrm{g} / \mathrm{mL})$ or FITC-conjugated anti-P-selectin antibody $(5 \mu \mathrm{g} / \mathrm{mL})$, as described before. ${ }^{4}$ For detection of apoptotic phosphatidylserine (PS) exposure, washed platelets $\left(10 \times 10^{6} / \mathrm{mL}\right)$ were stimulated with ABT-737 $(5-10 \mu \mathrm{M})$ for 150 min. ${ }^{5}$ Sub-samples were stained with FITC-annexin A5 $(0.25 \mu \mathrm{g} / \mathrm{mL})$ in the presence of $2 \mathrm{mM} \mathrm{CaCl}_{2}$. Platelets were pretreated with inhibitor or vehicle for $15 \mathrm{~min}$, where indicated. Samples were analyzed using a BD Accuri C6 flow cytometer and software (Becton-Dickinson Bioscience, Franklin Lakes, NJ, USA). ${ }^{5}$

To assess intracellular $\mathrm{Ca}^{2+}$ rises, washed platelets were loaded with the $\mathrm{Ca}^{2+}$ probe Fluo-4 AM $(8 \mu \mathrm{M})$ in the presence of Pluronic F-127 $(0.4 \mu \mathrm{g} / \mathrm{mL})$ for $45 \mathrm{~min}$, as described. ${ }^{5}$ After removal of excess probe by a wash step, Fluo-4-loaded platelets were resuspended into Hepes buffer $\mathrm{pH} 7.45\left(2 \times 10^{6} / \mathrm{mL}\right)$ supplemented with $2 \mathrm{mM} \mathrm{CaCl}_{2}$, and then activated with thrombin $(4 \mathrm{nM}), \mathrm{CRP}-\mathrm{XL}(10 \mu \mathrm{g} / \mathrm{mL})$ or the SERCA pump inhibitor thapsigargin $(0.5 \mu \mathrm{M})$. Fluorescence changes were monitored in time, and expressed as increases over basal fluorescence, indicative of rises in cytosolic $\mathrm{Ca}^{2+}$ concentrations. ${ }^{5}$

\section{Platelet spreading on fibrinogen}

Washed glass coverslips were coated with fibrinogen $(100 \mu \mathrm{g} / \mathrm{mL})$ and blocked with $1 \%$ BSA. Washed platelets $\left(10 \times 10^{6} / \mathrm{mL}\right)$ were allowed to adhere and spread for $10 \mathrm{~min},{ }^{6}$ after which brightfield images were captured using a Zeiss LSM7 confocal line-scanning microscope (Oberkochen, Germany) equipped with a $63 x$ oil immersion objective. ${ }^{7}$

\section{Mitochondrial function and morphology}

To detect mitochondrial depolarization, washed platelets from patients and controls $\left(10 \times 10^{6} / \mathrm{mL}\right)$ were loaded with the mitochondrial membrane potential dye, tetramethylrhodamine, ethyl ester (TMRE, $50 \mathrm{nM}$ ) for $30 \mathrm{~min}$ at room temperature. Using flow cytometry, mean fluorescence intensities of TMRE were assessed to evaluate the mitochondrial membrane potential. ${ }^{5}$

Mitochondrial respiration was determined by high-resolution respirometry with an Oroboros Oxygraph-2K (Oroboros Instruments, Innsbruck, Austria), at $37^{\circ} \mathrm{C}$ and roomair saturated oxygen tension, according to previously established methodology. ${ }^{8}$ Briefly, washed platelets were resuspended in buffer Z (105 mM K-MES, $30 \mathrm{mM} \mathrm{KCl,} 10 \mathrm{mM}$ $\mathrm{KH}_{2} \mathrm{PO}_{4^{\prime}} 1 \mathrm{mM}$ EGTA, $5 \mathrm{mM} \mathrm{MgCl}, 5 \mathrm{mg} / \mathrm{mL}$ BSA, $5 \mu \mathrm{M}$ pyruvate and $2 \mu \mathrm{M}$ malate, $\mathrm{pH}$ 7.1) and allowed to stabilize on ice for $30 \mathrm{~min}$. Washed platelets (final concentration: $50 \mathrm{x}$ $10^{6} / \mathrm{mL}$ ) were added to the respiration chamber, which contained buffer $Z$, supplemented with $5 \mathrm{mM}$ pyruvate, $0.5 \mathrm{mM}$ malate and $50 \mu \mathrm{g} / \mathrm{mL}$ saponin. After approximately $15 \mathrm{~min}$, 
when stable respiration was reached, the OXPHOS substrates ADP ( $5 \mathrm{mM})$, glutamate $(10 \mathrm{mM})$, succinate $(10 \mathrm{mM})$ and cytochrome $\mathrm{c}(5 \mathrm{mM})$ were sequentially added in 10 min increments. Oxygen concentration and oxygen flux (the negative time derivative of oxygen consumption) were monitored in real time.

As an independent read-out of mitochondrial content, citrate synthase activity was determined in lysates of washed platelets $\left(5 \times 10^{6}\right)$, using a colorimetric assay (Sigma, St. Louis, MO, USA). Enzymatic activity was determined at $37^{\circ} \mathrm{C}$ from absorbance changes at $412 \mathrm{~nm}$, according to the manufacturer's protocol.

For transmission electron microscopy, washed platelets in the presence of $10 \mathrm{nM}$ iloprost were fixed during $1 \mathrm{~h}$ with $1.5 \%$ glutaraldehyde in phosphate-buffered saline. Samples were then embedded in Epon and processed, as described before. ${ }^{9}$

\section{Western blot analysis}

Washed platelets in Hepes buffer 7.45 were lysed (1:4) into $4 x$ ice-cold lysis buffer (600 mM NaCl, 40 mM Tris, 4 mM EGTA, 4 mM EDTA, 4\% Nonidet-P40, pH 7.5). Protein content was quantified using a micro BCA protein assay (Thermo Scientific, Waltham, MA, USA). Samples were denatured by adding 1:4 NuPAGE LDS sample buffer, and subsequently heated at $95^{\circ} \mathrm{C}$ for $5 \mathrm{~min}$. The denatured samples were separated on an $8 \%$ SDS-polyacrylamide gel, and then transferred to a PVDF blotting membrane. Immunoblotting was performed with antibodies against kindlin-3 (1:1000) or $\alpha$-tubulin (1:1000; loading control) overnight at $4{ }^{\circ} \mathrm{C}$, followed by staining using a horseradish peroxidase (HRP) coupled secondary antibody for $1 \mathrm{~h}$ at room temperature. After incubation with SuperSignal West pico-chemiluminescent substrate, protein bands were visualized using an ImageQuant LAS-4000 mini system (Wauwatosa, WI, USA).

\section{Caspase activity}

Caspase activity was assessed in washed platelets $\left(10 \times 10^{6} / \mathrm{mL}\right)$ treated with vehicle or ABT-737 $(10 \mu \mathrm{M})$ for $90 \mathrm{~min}$ at $37^{\circ} \mathrm{C}$, as described before. ${ }^{5}$

\section{Whole blood thrombus formation}

Whole blood perfusion assays were performed according to published procedures. ${ }^{7}$ In short, washed glass coverslips were coated with $1.0 \mu \mathrm{L}$ microspots, containing collagen type I $(50 \mu \mathrm{g} / \mathrm{mL})$ with or without tissue factor $(500 \mathrm{pM})$, and then blocked with $1 \% \mathrm{BSA}$. Citrated whole blood supplemented with AF546-labeled human fibrinogen $(15 \mu \mathrm{g} / \mathrm{mL})$ was co-perfused (9:1, vol./vol.) with coagulation buffer (Hepes buffer pH 7.45, $63 \mathrm{mM}$ $\mathrm{CaCl}_{2}, 32 \mathrm{mM} \mathrm{MgCl}_{2}$ ) over the coverslips, at a wall shear rate of $1000 \mathrm{~s}^{-1}$ for $10 \mathrm{~min}$. After blood perfusion, thrombi were rinsed for 2 min with Hepes buffer $\mathrm{pH} 7.45$ to remove red blood cells. Microscopic bright field and fluorescence images (1360x1024 pixels, 142×107 $\mu \mathrm{m}$ ) were then collected using an EVOS microscope (Life Technologies, Bleiswijk, The Netherlands), equipped with a $60 x$ oil objective. Image analysis was performed using the program Fiji. ${ }^{10}$ 


\section{Supplemental Results}

Table S1: Chemotherapeutic drugs taken by included patients, classified according to the main action mechanism. Drug classes: A, antitumor antibiotics \& topo-isomerase II inhibitors; B, antimetabolites; C, alkylating agents; D, mitotic inhibitors; $E$, other.

\begin{tabular}{|l|l|}
\hline Class & Drug \\
\hline A & daunorubicin, doxorubicin, etoposide, mitoxantrone, idarubicin \\
\hline B & cytarabine (ara-C), fludarabine \\
\hline C & busulfan, carmustine (BCNU), cyclofosfamide, melphalan \\
\hline D & vincristin \\
\hline E & lenalidomide, tosedostat, retuximab \\
\hline
\end{tabular}

Table S2: Numbers of included patients taking chemotherapeutic drugs of different classes, alone or in combination. For the list of individual drugs, see Table S1.

\begin{tabular}{|l|c|c|c|c|c|}
\hline Diagnosis & \multicolumn{5}{|c|}{ Drug class } \\
\hline & $\begin{array}{c}\mathrm{A} \\
\text { anti- } \\
\text { tumor \& TI } \\
\text { inhibitor }\end{array}$ & $\begin{array}{c}\mathrm{B} \\
\text { anti- } \\
\text { metabolite }\end{array}$ & $\begin{array}{c}\text { C } \\
\text { alkylating } \\
\text { agent }\end{array}$ & $\begin{array}{c}\text { D } \\
\text { mitotic } \\
\text { inhibitor }\end{array}$ & $\begin{array}{c}\mathrm{E} \\
\text { other }\end{array}$ \\
\hline AML/ALL, $n$ & 24 & 29 & 10 & 1 & 10 \\
\hline Lymphoma, $n$ & 12 & 14 & 14 & 1 & 1 \\
\hline Multiple Myeloma, $\mathrm{n}$ & 0 & 0 & 16 & 0 & 0 \\
\hline Other, $n$ & 0 & 1 & 3 & 0 & 0 \\
\hline Total, $n$ (\%) & $36(26.5 \%)$ & $44(32.3 \%)$ & $43(31.6 \%)$ & $2(1.5 \%)$ & $11(8.1 \%)$ \\
\hline
\end{tabular}


Table S3: Hematologic parameters of blood samples from patients before and $1 \mathrm{~h}$ after platelet transfusion.

Data are expressed as medians (interquartile ranges)

\begin{tabular}{|l|l|l|}
\hline Parameter & Before transfusion & After transfusion \\
\hline Leukocyte count $\left(\times 10^{9} / \mathrm{L}\right)$ & $0.14(0.04-0.41)$ & $0.12(0.04-0.44)$ \\
\hline Hemoglobin $(\mathrm{mM})$ & $5.6(5.1-6.2)$ & $5.2(4.7-5.7)$ \\
\hline Platelet count $\left(\times 10^{9} / \mathrm{L}\right)$ & $6(4-8)$ & $33(24.3-43.8)$ \\
\hline Absolute immature platelet number $\left(\times 10^{9} / \mathrm{L}\right)$ & $0.21(0.07-0.38)$ & $1.17(0.69-1.60)$ \\
\hline Immature platelet fraction $(\%)$ & $3.8(2.2-6.3)$ & $3.6(2.7-4.2)$ \\
\hline Corrected count increment & n.a. & $14.8(11.3-18.0)$ \\
\hline
\end{tabular}

Table S4: Coagulation parameters and factor activities of patient blood samples. Factor activities were assayed relatively to Standard Human Plasma (SHP) and are expressed as \% activity. Samples without prior platelet transfusion. (Medians + interquartile ranges)

\begin{tabular}{|l|l|l|}
\hline Coagulation parameters & Patient cohort & Reference range \\
\hline Fibrinogen (g/L) & $4.2(3.3-5.1)$ & $1.7-4.0$ \\
\hline d-Dimer (ng/mL) & $2198(1400-4391)$ & $<500$ \\
\hline aPTT (s) & $28.6(26.4-33.9)$ & $23.0-32.0$ \\
\hline PT (s) & $11.1(10.6-11.8)$ & $9.9-11.5$ \\
\hline Thrombin time (s) & $16.0(15.1-16.8)$ & $15.0-22.0$ \\
\hline vWF (\%) & $211(154-296)$ & $40-190$ \\
\hline Prothrombin (\%) & $96(84-101)$ & $70-130$ \\
\hline Factor V (\%) & $123(103-144)$ & $70-130$ \\
\hline Factor VII (\%) & $60(47-75)$ & $70-130$ \\
\hline Factor VIII (\%) & $163(142-194)$ & $50-200$ \\
\hline Factor IX (\%) & $127(115-162)$ & $60-140$ \\
\hline Factor X (\%) & $83(73-89)$ & $60-140$ \\
\hline Factor XI (\%) & $99(88-117)$ & $60-140$ \\
\hline Factor XII (\%) & $86(75-107)$ & $60-140$ \\
\hline Factor XIII (\%) & $93(86-110)$ & $70-140$ \\
\hline
\end{tabular}




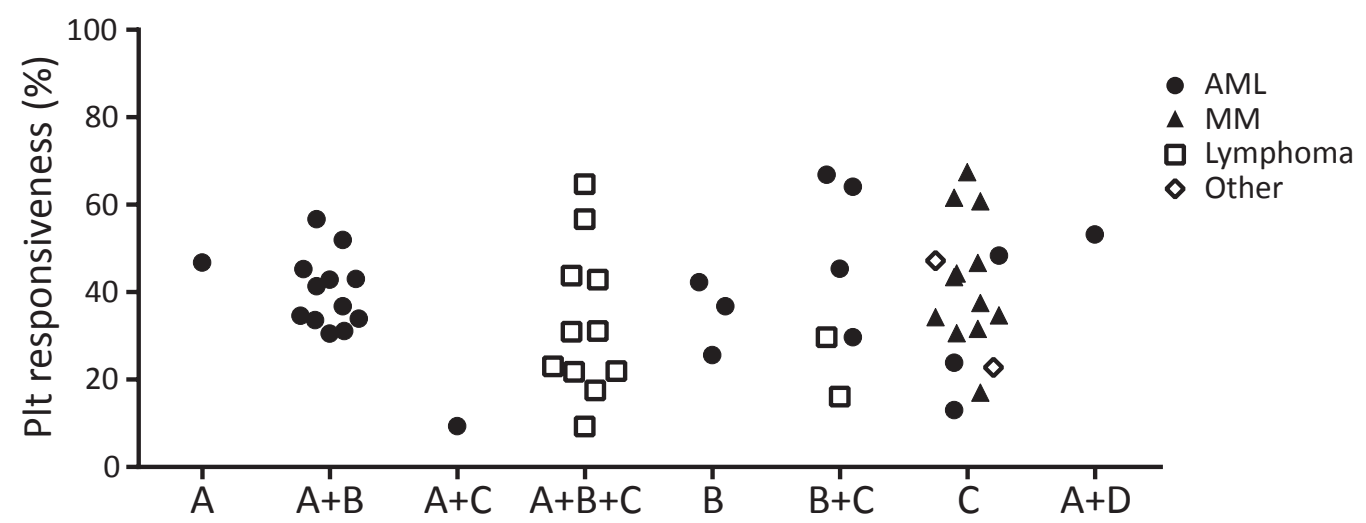

Figure S1: Variable impairment in platelet responsiveness is independent of chemotherapy treatment regimen of patients. Integrin activation and P-selectin expression of washed platelets from patients was determined, as for Figure 1. Overall platelet responsiveness was defined per patient as the mean of percentages of platelets positive for integrin activation and P-selectin after stimulation with thrombin, CRP$\mathrm{XL}$ or ADP. Patients were grouped based on treatment with chemotherapeutics into one of more classes (see Table S1): A, antitumor antibiotics \& topo-isomerase inhibitors; B, antimetabolites; C, alkylating agents; D, alkaloids. Data from 52 patients (AML: $n=25$, multiple myeloma: $n=12$, lymphoma: $n=13$, other: $n=2$ ). 
A
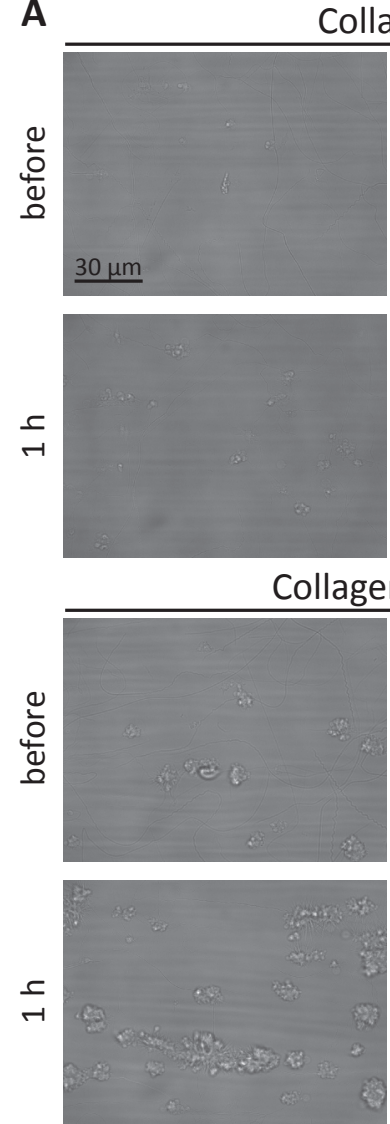

Brightfield

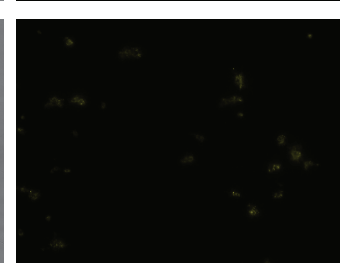

Collagen

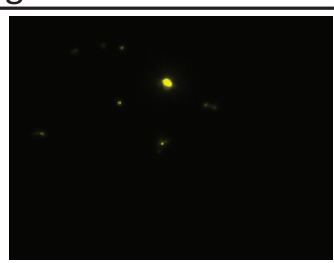

$n+T F$
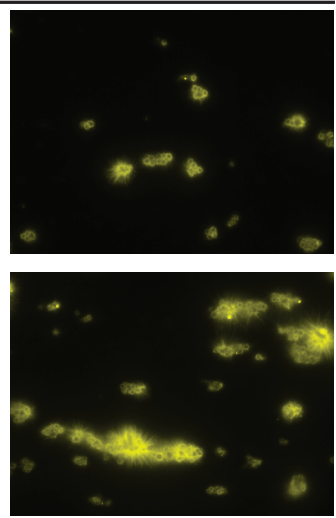

AF546- fibrinogen
B

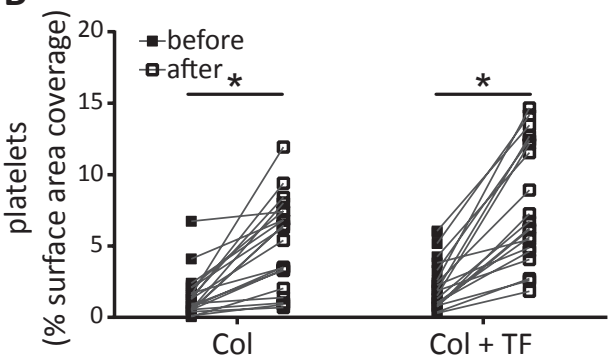

C

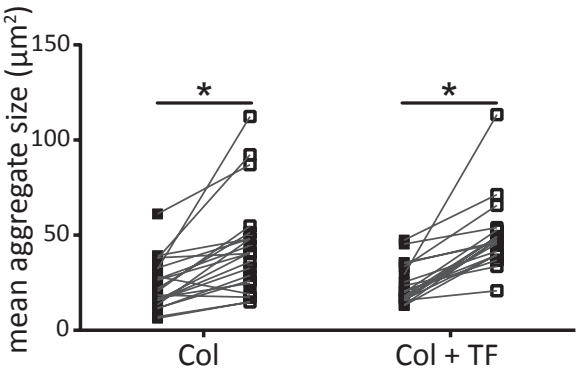

D

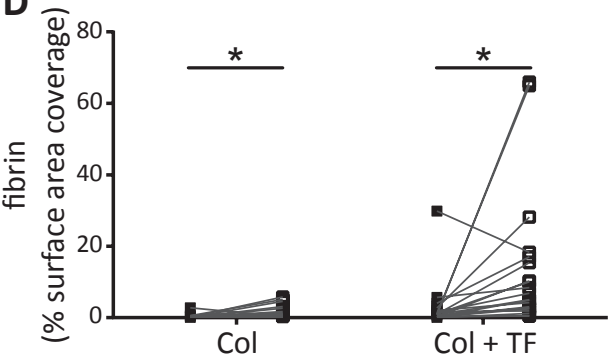

Figure S2: Effect of platelet transfusion on thrombus formation and platelet-dependent fibrin formation in whole blood from patients. Blood samples were obtained from 23 patients (treatment classes: A+B: $n=7$; $\mathrm{A}+\mathrm{B}+\mathrm{C}: n=6 ; \mathrm{B}+\mathrm{C}: n=2 ; \mathrm{C}: n=5$ ) before and $1 \mathrm{~h}$ after platelet transfusion (median count of concentrate $363 \mathrm{x}$ $10^{6}$ platelets $/ \mathrm{mL}$, IQR: $332-397 \times 10^{6} / \mathrm{mL}$ ), and used for assessment of formation of platelet-fibrin thrombi. Therefore, citrated whole blood samples were recalcified using a dual inlet system, and perfused over two spots consisting of collagen I and collagen I plus tissue factor. After $10 \mathrm{~min}$ of perfusion, microscopic images were captured. A) Representative brightfield and fluorescence images before and after platelet transfusion. In addition, quantification of B) platelet adhesion (expressed as percentage surface area coverage); C) mean aggregate size; D) fibrin formation (surface area coverage). $n=23$ patients, ${ }^{*} p<0.05$. Overall platelet responsiveness of patients included in the figure: $24.7-42.4 \%$ (IQR). 


\section{References}

1. Cauwenberghs S, Feijge MA, Hageman G, Hoylaerts M, Akkerman JWN, Curvers J, et al. Plasma ectonucleotidases prevent desensitization of purinergic receptors in stored platelets. Transfusion. 2006;46:101828.

2. van Beers JJ, van Egmond LT, Wetzels RJ, Verhezen PW, Beckers EA, van Oerle R, et al. Increased coagulation and fibrinolytic potential of solvent-detergent plasma: a comparative study between Omniplasma and fresh frozen plasma. Vox Sang. 2016;111(1):33-42.

3. Schols SE, Lancé MD, Feijge MA, Damoiseaux J, Marcus MA, Hamulyák K, et al. Impaired thrombin generation and fibrin clot formation in patients with dilutional coagulopathy during major surgery. Thromb Haemost. 2010;103(2):318-28.

4. Mattheij NJ, Gilio K, van Kruchten R, Jobe SM, Wieschhaus AJ, Chishti AH, et al. Dual mechanism of integrin aiibb3 closure in procoagulant platelets. J Biol Chem. 2013;288(19):13325-36.

5. van Kruchten R, Mattheij NJA, Saunders C, Feijge MA, Swieringa F, Wolfs JLN, et al. Both TMEM16Fdependent and TMEM16F-independent pathways contribute to phosphatidylserine exposure in platelet apoptosis and platelet activation. Blood. 2013;121(10):1850-7.

6. Nergiz-Unal R, Lamers MM, van Kruchten R, Luiken JJ, Cosemans JM, Glatz JF, et al. Signaling role of CD36 in platelet activation and thrombus formation on immobilized thrombospondin or oxidized low-density lipoprotein. J Thromb Haemost. 2011;9(9):1835-46.

7. Swieringa F, Baaten CC, Verdoold R, Mastenbroek TG, Rijnveld N, van der Laan KO, et al. Platelet control of fibrin distribution and microelasticity in thrombus formation under flow. Arterioscler Thromb Vasc Biol. 2016;36:692-9.

8. Sjövall F, Morota S, Hansson MJ, Friberg H, Gnaiger E, Elmér E. Temporal increase of platelet mitochondrial respiration is negatively associated with clinical outcome in patients with sepsis. Crit Care. 2010;14(6):R214. 9. van Nispen tot Pannerden H, de Haas F, Geerts W, Posthuma G, van Dijk S, Heijnen HF. The platelet interior revisited: electron tomography reveals tubular alpha-granule subtypes. Blood. 2010;116(7):1147-56. 10. Schindelin J, Arganda-Carreras I, Frise E, Kaynig V, Longair M, Pietzsch T, et al. Fiji: an open-source platform for biological-image analysis. Nat Methods. 2012;9(7):676-82. 




\section{Chapter 7}

Additive roles of platelets and fibrinogen in whole blood fibrin clot formation upon dilution as assessed by thromboelastometry

Ninivaggi M, Feijge MA*, Baaten CC*, Kuiper GJ, Marcus MA, Ten Cate $H$, Lancé MD, Heemskerk JW, van der Meijden PE (*equal contribution)

Thromb Haemost. 2014; 111 (3): 447-457

Reprinted with permission 


\section{Abstract}

Background and aim: Blood dilution after transfusion fluids leads to diminished coagulant activity monitored by rotational thromboelastometry, assessing elastic fibrin clot formation, or by thrombin generation testing. We aimed to determine the contributions of blood cells (platelets, red blood cells) and plasma factors (fibrinogen, prothrombin complex concentrate) to fibrin clot formation under conditions of hemodilution in vitro or in vivo. Methods: Whole blood or plasma diluted in vitro was supplemented with platelets, red cells, fibrinogen or prothrombin complex concentrate (PCC). Thromboelastometry was measured in whole blood as well as plasma; thrombin generation was determined in parallel. Similar tests were performed with blood from 48 patients, obtained before and after massive fluid infusion during cardiothoracic surgery. Results: Addition of platelets or fibrinogen, in additive and independent ways, reversed the impaired fibrin clot formation (thromboelastometry) in diluted whole blood. In contrast, supplementation of red blood cells or prothrombin complex concentrate was ineffective. Platelets and fibrinogen independently restored clot formation in diluted plasma, resulting in thromboelastometry curves approaching those in whole blood. In whole-blood from patients undergoing dilution during surgery, elastic clot formation was determined by both the platelet count and the fibrinogen level. Thrombin generation in diluted (patient) plasma was not changed by fibrinogen, but improved markedly by prothrombin complex concentrate. Conclusions: In dilutional coagulopathy, platelets and fibrinogen independently, but not red blood cells or vitamin K-dependent coagulation factors, determine thromboelastometry parameters measured in whole-blood and plasma. Clinical decisions for transfusion based on thromboelastometry should take into account the platelet concentration.

\section{Introduction}

It is still a matter of debate how insufficiencies of the hemostatic system lead to bleeding under conditions of massive fluid infusion during cardiothoracic surgery. Fluid infusion diminishes a variety of processes, including platelet activity, coagulation factor levels, thrombin generation and fibrin clot formation. The latter process is monitored by rotational thromboelastometry or thromboelastography, which are frequently used to monitor patients during surgery. ${ }^{1-3}$ Elastic fibrin clot formation can be measured in whole blood samples, thus providing a rapid point-of-care method for goal-directed coagulation management of surgery or trauma patients with, for example, fibrinogen concentrate. ${ }^{4}$

Recently, we have shown that combined plasma measurement of thrombin generation and fibrin clot formation results in a better prediction of the bleeding risk after major surgery than the use of either test alone. ${ }^{5}$ The rationale is that thrombin generation monitors the rate and extent of formed thrombin, as a central controlling enzyme of the coagulation cascade. ${ }^{6}$ On the other hand, thromboelastometry, being more sensitive for fibrinogen ${ }^{7,8}$ and platelets, 8,9 reports on the more advanced stage of elastic fibrin clot formation. In patients with bleeding during major surgery or experiencing trauma, impaired hemostasis can be restored by administration of fibrinogen ${ }^{10-12}$ or vitamin K-dependent coagulation factors (prothrombin complex concentrate, PCC). ${ }^{10,13,14}$ 
Both types of concentrates are also effective in large animal models of injury-induced bleeding, ${ }^{15-17}$ suggesting that normalization of part of the coagulation factors helps to stop bleeding after dilution.

Vitamin K-dependent coagulation factors, platelets and fibrinogen all contribute to the process of elastic clot formation by providing enzymatic activity, strength and mass of a clot, respectively. However, it is still unresolved how, under conditions of dilutional coagulopathy, reduced levels of these blood components interact to limit fibrin clot formation, nor is it clear how red blood cells are involved in this process. In the present paper we aimed to determine these interactions. Using thromboelastometry, we measured fibrin clot formation in whole blood and plasma under conditions of dilution in vitro or in vivo, and assessed the effects of reconstitution of blood cells (platelets, red blood cells) and plasma coagulation factors (fibrinogen, PCC). The results show that the clot-forming process is controlled by platelets and fibrinogen in additive and independent ways, implicating that the platelet count is an important functional variable, independently of fibrinogen, in whole-blood thromboelastometry under condition of in vivo dilution in patients undergoing cardiothoracic surgery.

\section{Materials and Methods}

\section{Materials}

Bovine serum albumin (BSA) and apyrase were obtained from Sigma (St. Louis MO, USA). Human thrombin calibrator and thrombogram software were from Thrombinoscope (Maastricht, The Netherlands), thrombin substrate Z-Gly-Gly-Arg aminomethyl coumarin (Z-GGR-AMC) was from Bachem (Bubendorf, Switzerland). Thromboelastometry International (Munich, Germany) supplied the corresponding hardware, software and cuvettes.

Fibrinogen concentrate (Haemocomplettan P) and prothrombin complex concentrate (PCC, Beriplex) were from CSL-Behring (Marburg, Germany). PCC contains the vitamin K-dependent coagulation factors, prothrombin and factor VII, IX and X, and the anticoagulant factors, protein $\mathrm{C}$ and $\mathrm{S}$, and antithrombin/heparin; $1 \mathrm{U} / \mathrm{mL}$ PCC refers to $100 \%$ prothrombin. Recombinant tissue factor (Innovin) was from Dade Behring (Deerfield IL, USA). Phospholipid vesicles containing phosphatidylserine, phosphatidylcholine and phosphatidylethanolamine (20:60:20) were purchased from Avanti (Alabaster AL, USA) and prepared by ultrasonication. ${ }^{18}$ All other reagents came from sources described before. ${ }^{5}$

\section{Blood donors}

The studies were approved by the local medical ethics committee (MEC 07-2-114). Healthy donors and patients gave full informed consent for blood donation, according to the Helsinki declaration. Healthy subjects had not taken antithrombotic medication for at least two weeks. For in vitro dilution experiments, blood was obtained from 15 healthy subjects by venipuncture using a $1.2 \mathrm{~mm}$ needle, allowing the blood to drip freely into open tubes (first 2-3 mL were discarded). The collection tubes contained either 1/10 
volume of trisodium citrate $(0.129 \mathrm{M})$ for the preparation of platelet-free plasma; or $1 / 6$ volume of acidic citrate dextrose (ACD: $80 \mathrm{mM}$ trisodium citrate, $52 \mathrm{mM}$ citric acid and $180 \mathrm{mM}$ glucose) for the isolation of washed platelets.

Blood samples were obtained from 48 patients before and after a cardiopulmonary bypass (CPB) procedure. This sample size is based on the results of an earlier study, where the effects of in vivo dilution on elastic fibrin clot formation in only plasma were determined. ${ }^{19}$ Patients were admitted in the hospital in the period of October to December 2010. Patients had stopped taking anticoagulant drugs at least 1 week before the procedure. Blood samples were collected into $0.129 \mathrm{M}$ trisodium citrate (1:10) Vacuette tubes (Greiner, Alphen a/d Rijn, The Netherlands) at two time points: (i) after induction of anesthesia, but prior to the CPB procedure and heparin administration; (ii) after surgery and infusion of protamine to neutralize heparin, when the activated clotting time (ACT) was normalized. In samples from 4 patients coagulation times pointed to the presence of residual traces of heparin. In these cases, assays were repeated in the presence of $10 \mu \mathrm{g} / \mathrm{mL}$ polybrene, i.e. a concentration not influencing the assay parameters (data not shown). None of the patients developed surgical complications other than bleeding during the time span of blood sampling. Transfusion of blood products during surgery was guided on the basis of low blood cell counts, prolonged aPTT, and/or clinical observation of bleeding. Whole blood thromboelastometry was measured immediately after collection; plasma samples were stored for later measurements.

\section{Preparation of plasma, washed platelets and red blood cells}

Platelet-free plasma was obtained from citrate-anticoagulated blood, by centrifuging twice at $2630 \mathrm{~g}$ for $10 \mathrm{~min} .{ }^{20}$ Plasma samples were immediately snap-frozen into liquid nitrogen, and stored at $-80^{\circ} \mathrm{C}$ until further use. Washed platelets were prepared from ACD-anticoagulated blood, and suspended in Hepes buffer pH 7.45 (10 mM Hepes, $136 \mathrm{mM} \mathrm{NaCl}, 2.7 \mathrm{mM} \mathrm{KCl}, 2 \mathrm{mM} \mathrm{MgCl}, 0.1 \%$ glucose and $0.1 \% \mathrm{BSA}$ ), as described. ${ }^{19}$ Platelet count was determined with a thrombocounter (Coulter Electronics, Luton, UK). For the isolation of red blood cells, citrate-anticoagulated blood was centrifuged at $240 \mathrm{~g}$ for $15 \mathrm{~min}$, after which the red cell layer was supplemented with Hepes buffer $\mathrm{pH} 7.45$ in a 1:2 volume ratio. Red cells were then centrifuged at $2630 \mathrm{~g}$ for $10 \mathrm{~min}$, suspended with Hepes buffer $\mathrm{pH} 7.45$ (2:1), and washed again, which yielded a highly purified suspension of red cells (99.9\%).

\section{Dilution of whole blood or plasma and reconstitution experiments}

Whole blood or plasma was diluted in vitro with saline medium, consisting of 137 $\mathrm{mM} \mathrm{NaCl}, 12.9 \mathrm{mM}$ trisodium citrate, $2 \mathrm{mM} \mathrm{CaCl}_{2}$ and $2 \mathrm{mM} \mathrm{MgCl}_{2}$, in order to keep equal concentrations of free $\mathrm{Ca}^{2+}$ and $\mathrm{Mg}^{2+}$ in all diluted samples. Coagulation factor levels in plasma were determined, as described. ${ }^{5}$ Percentage values of plasma are given as final concentrations (relative to citrate-anticoagulated plasma taken as $100 \%$ ). In all reconstitution experiments, replacement of plasma by added supplements was taken into account when calculating the final extent of dilution. Red blood cells were added at 
1:10 volume ratio. Where indicated, factor concentrates in saline were added to plasma samples from healthy subjects or patients, and incubated for $10 \mathrm{~min}$ before starting the experiment.

\section{Rotational thromboelastometry}

In samples from the same donors, elastic fibrin clot formation was measured in whole blood and plasma (citrate-anticoagulated) by thromboelastometry, using equipment and cuvettes from TEM International. Coagulation was triggered with 10 $\mathrm{pM}$ tissue factor and a surplus of $\mathrm{CaCl}_{2} \cdot{ }^{19}$ Plasma samples were supplemented with phospholipid vesicles ( $4 \mu \mathrm{M}$, final concentration) or washed platelets from one healthy donor (100-250 $\times 10^{9} / \mathrm{L}$, final count). Runs were performed in duplicate or, when $>5 \%$ variation was observed, in triplicate. Curves were analyzed for slope of elastic clot formation ( $\alpha$-angle), maximum strength of the clot (maximal clot firmness, MCF) and the time to the onset of clot formation (clotting time, CT).

\section{Thrombin generation}

Thrombin generation in plasma was measured, using the calibrated automated thrombogram (CAT) method. ${ }^{19}$ Plasma samples were supplemented with either phospholipid vesicles $(4 \mu \mathrm{M})$ or washed platelets from a single healthy donor (100-250 $\times$ $10^{9} / \mathrm{L}$ ). Assays were run in 96-well U-bottom plates (Milford, MA, USA) in the presence of fluorogenic substrate Z-GGR-AMC $(2.5 \mathrm{mM})$ and $\mathrm{CaCl}_{2}(16.7 \mathrm{mM}$, final concentrations). Coagulation was triggered with $10 \mathrm{pM}$ tissue factor. Measurements were performed in triplicate. First-derivate curves of thrombin generation were obtained by using Thrombogram software. ${ }^{20}$ Curves were analyzed for maximal rate of thrombin generation (thrombin peak height), thrombin-generating capacity (endogenous thrombin potential, ETP) and the time until thrombin formation (lag time). Samples containing added PCC were analyzed for $10 \mathrm{~min}$ after triggering coagulation, to ensure adequate curve calibration.

\section{Statistical analysis}

Data are given as means \pm SD. The Shapiro-Wilk test was used to test for normal distribution of the data. Statistical analysis was performed, as appropriate, using the Mann-Whitney $U$ test. Patient data were not normally distributed and are given as medians with interquartile ranges. The Wilcoxon matched pairs signed ranked test was used for comparing pre- and post-surgical values. Determinants of thromboelastometry curves were identified by multiple linear regression analysis. P-values $<0.05$ were considered statistically significant. The program GraphPad Prism 6.0 (GraphPad Software, San Diego, CA, USA) was used for graphical purposes and relevant statistics; the SPSS 20 (IBM, Armonk, NY, USA) package was used for regression analysis and statistical testing. 


\section{Results}

Determinants of thromboelastometry and thrombin generation curves for whole blood and plasma upon in vitro dilution

Blood samples from healthy subjects were diluted in vitro, reconstituted with various blood components, and analyzed for elastic fibrin clot formation by thromboelastometry. Coagulation was triggered via the extrinsic pathway with recombinant human tissue factor at an optimal concentration of $10 \mathrm{pM} .{ }^{19}$ To keep cationic concentrations constant, citrate-anticoagulated blood samples were diluted with saline medium, containing equimolar concentrations of citrate, $\mathrm{CaCl}_{2}$ and $\mathrm{MgCl}_{2}$. The $\mathrm{MCF}$, as a curve parameter determining the overall clotting capacity, was half-maximal at $30 \%$ blood, while the $\alpha$-angle, detecting the rate of elastic clot formation, was half-maximal at $20 \%$ blood (Fig. 1A). The clotting time (CT) was prolonged from $<40 \%$ blood (Fig. 1B). Strikingly,

A

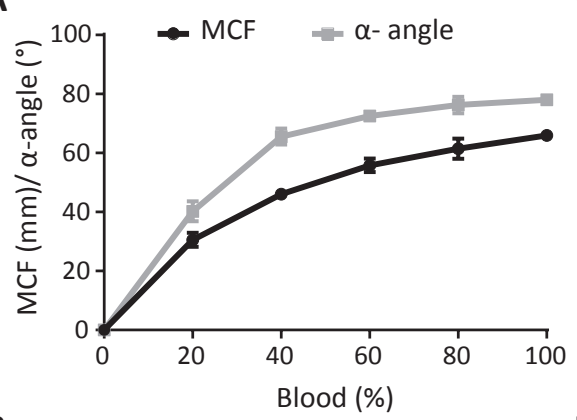

B

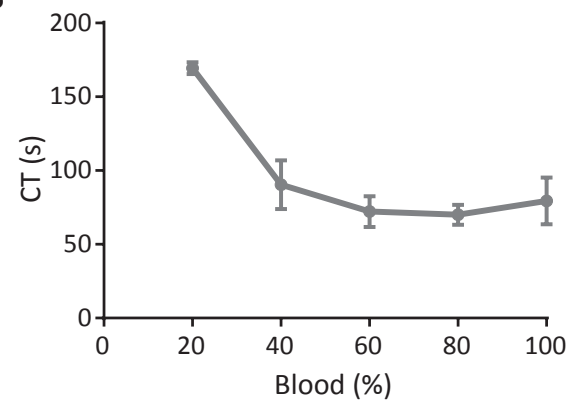

C
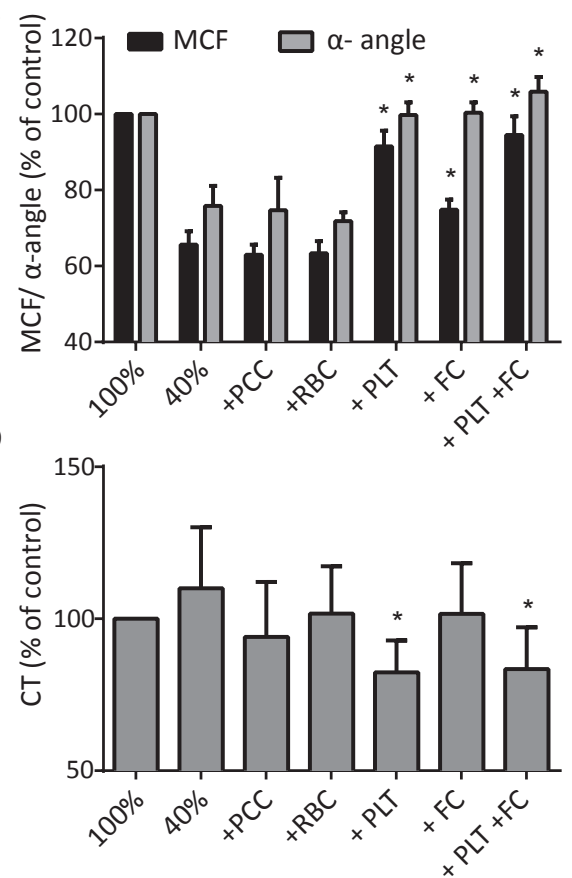

Figure 1: Determinants of thromboelastometry in diluted whole blood. Whole blood samples from healthy subjects were diluted with saline medium (see methods) to the indicated percentage. Levels of hemostatic factors in the undiluted blood (plasma) were: platelets $220-250 \times 10^{9} / \mathrm{L}$; red blood cells $33-37 \%$ hematocrit; fibrinogen 3.0-3.5 g/L, prothrombin 100\%. (A, B) Effect of blood dilution on maximal clot firmness (MCF), $\alpha$-angle and clotting time (CT) of thromboelastometry curves. (C, D) Whole blood diluted to $40 \%$ was supplemented (final concentrations) with prothrombin complex concentrate (PCC $1 \mathrm{U} / \mathrm{mL}$, equivalent to $100 \%$ prothrombin), red blood cells (RBC, 37\% hematocrit), autologous platelets (PLT, $250 \times 10^{9} / \mathrm{L}$ ) and/or fibrinogen concentrate (FC, $3.0 \mathrm{~g} / \mathrm{L}$.). Values of MCF, $\alpha$-angle and CT are given in comparison to the control condition with undiluted blood (100\%). Means \pm SD $(n=5-8), * p<0.05$ vs. no addition. 

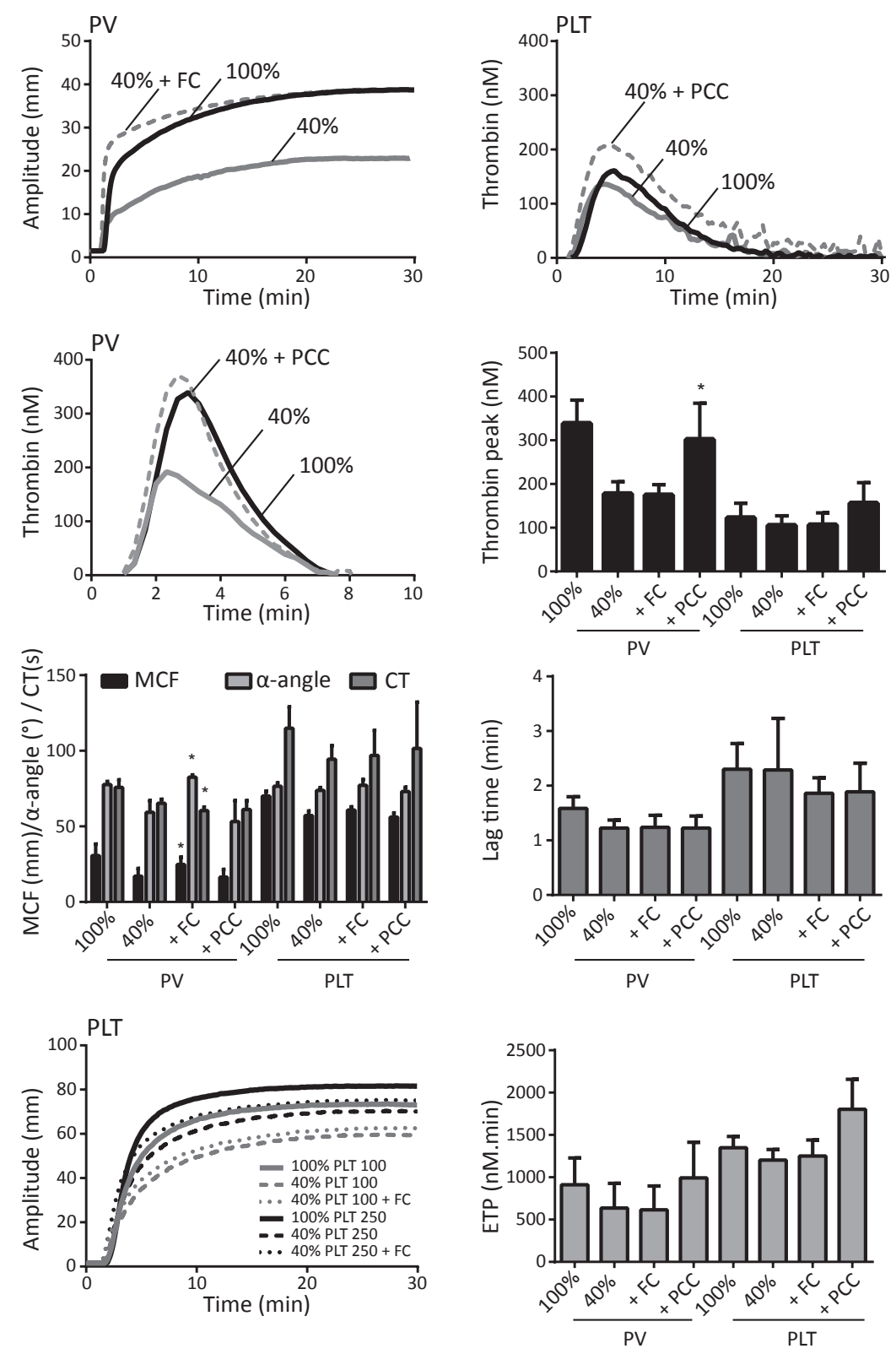

Figure 2: Determinants of thromboelastometry and thrombin generation in diluted plasma. Normal plasma (fibrinogen $3.2 \mathrm{~g} / \mathrm{L}$, prothrombin $100 \%$ ) was diluted with saline medium. Samples were replenished (final concentrations) with fibrinogen concentrate (FC, $3.0 \mathrm{~g} / \mathrm{L}$ ) or prothrombin complex concentrate (PCC, $100 \%$ prothrombin). Final plasma dilution of all samples was $40 \%$. Coagulation was triggered with $10 \mathrm{pM}$ tissue factor in the presence of either procoagulant phospholipid vesicles (PV, $4 \mu \mathrm{M}$ ) or platelets (PLT, 100 $\times 10^{9} /$ L). (A, C) Representative thromboelastometry curves with phospholipid vesicles or platelets. (B, D) Representative thrombin generation curves with phospholipid vesicles or platelets. (E-H) Effect of added fibrinogen or PCC on parameters of thromboelastometry $(E)$ and thrombin generation curves $(\mathbf{F}-\mathbf{H})$. Means $\pm \mathrm{SD}(n=5-6),{ }^{*} p<0.05$ vs. no addition. 
after dilution of blood to $40 \%$ (as in patients undergoing major surgery with bleeding), neither the MCF nor the $\alpha$-angle changed by addition of PCC or red blood cells (Fig. 1C). Addition of red blood cells to raise the hematocrit from 10 to $35 \%$ (at $40 \%$ plasma) did not influence thromboelastometry curve parameters (data not shown, but see below). In contrast, re-addition of either platelets (to $250 \times 10^{9} / \mathrm{L}$ ) or fibrinogen concentrate (to 3.0 $\mathrm{g} / \mathrm{L}$ ) to the diluted blood markedly restored parameters of fibrin clot formation (Fig. 1C). Only addition of platelets normalized the CT (Fig. 1D).

Given the inability of red cells to affect thromboelastometry curves, further experiments were performed with isolated plasma, as this allowed simultaneous measurements of elastic clot formation and thrombin generation. Coagulation was triggered again with $10 \mathrm{pM}$ tissue factor, while phospholipid vesicles $(4 \mu \mathrm{M})$ or autologous platelets $\left(100-250 \times 10^{9} / \mathrm{L}\right)$ were added to provide a phospholipid surface for coagulation activity.$^{19}$ In the presence of phospholipid vesicles, thromboelastometry curves remained low in slope ( $\alpha$-angle) and amplitude (MCF). Plasma dilution to $40 \%$ resulted in further lowering of the curves (Fig. 2A), and in diminished thrombin generation (Fig. 2B). Supplementation of fibrinogen concentrate, but not of $P C C$, antagonized the dilution effect on thromboelastometry (Fig. 2A, E). In contrast, only PCC markedly antagonized the dilution effect on thrombin generation (Fig. 2B, F-H). This analysis underscored the fibrinogen level as a major variable (independently of platelets) in elastic clot formation.

In the presence of platelets $\left(100 \times 10^{9} / \mathrm{L}\right)$, the rate and maximal amplitude of elastic clot formation were markedly higher and reached the maximal levels detected in whole blood (Fig. 2C, E), thus pointing to an additive effect of platelets to the thromboelastometry curves. In this case, plasma dilution to $40 \%$ only moderately diminished the thromboelastometry curves. In measurements of thrombin generation with platelets, plasma dilution was of limited effect on thrombin peak height, total amount of thrombin (ETP) or lag time (Fig. 2F-H).

Dose-response studies were performed to better define the effects of fibrinogen and PCC in diluted plasma. For thromboelastometry curves, added fibrinogen up to 3-4 g/L (final concentration) caused a dose-dependent increase in MCF and $\alpha$-angle, regardless of whether PCC was added or not (Fig. 3A, B). Added fibrinogen did not affect the CT, while only the highest concentration of PCC caused a slight prolongation in CT (Fig. 3C). Addition of PCC resulted in a dose-dependent increase in thrombin generation, reaching $300 \%$ of the normal thrombin peak height and ETP level. At none of the doses, fibrinogen influenced parameters of thrombin generation (Fig. 3D, E). However, PCC at high doses prolonged the lag time to thrombin formation (Fig. 3F), which can be explained by the presence of anticoagulant proteins in this concentrate. Together, these data indicate that, in diluted whole blood and plasma, fibrin clot formation relies on the amounts of platelets and fibrinogen, whereas the thrombin generation process is regulated by the levels of vitamin K-dependent coagulation factors, as present in PCC. 
A

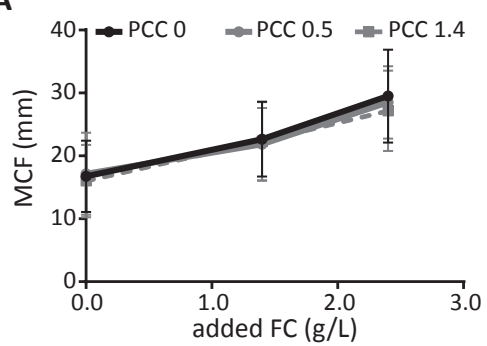

B
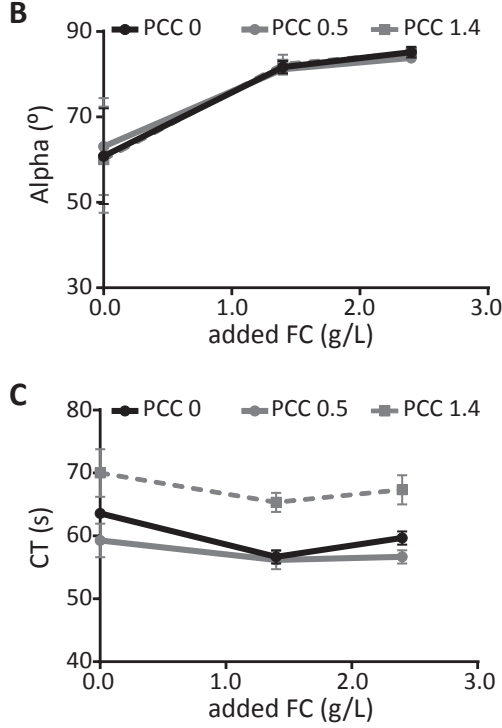

D

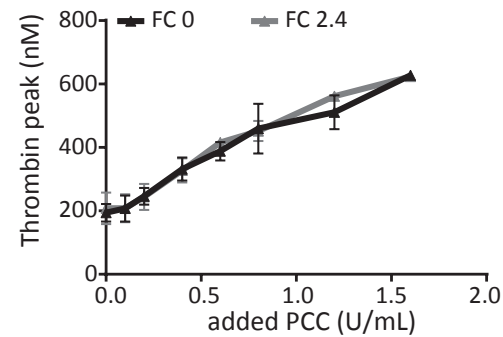

E

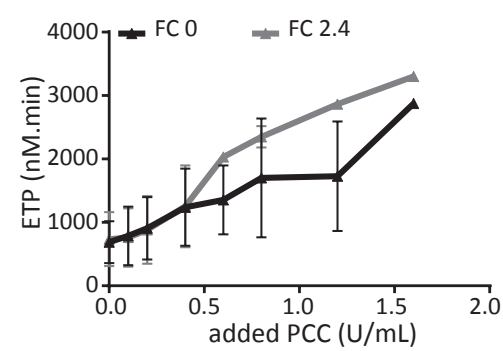

$\mathbf{F}$

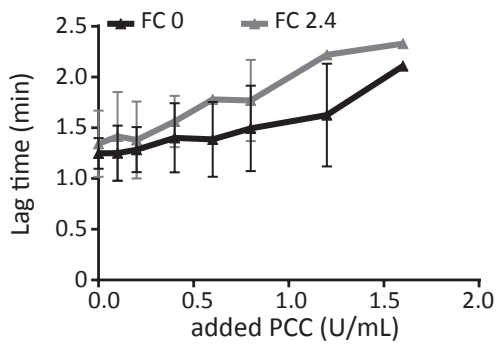

Figure 3: Dissimilar effects of fibrinogen and prothrombin complex concentrate on thromboelastometry and thrombin generation in diluted plasma. Normal plasma was diluted with saline medium and replenished with fibrinogen concentrate ( $F C, 1.4-2.4 \mathrm{~g} / \mathrm{L}$ ) and/or prothrombin complex concentrate (PCC, 0.1-1.6 U/mL). Final plasma dilution in all samples was $40 \%$. Coagulation was triggered with $10 \mathrm{pM}$ tissue factor in the presence of phospholipid vesicles. Effects of addition of different amounts of FC with or without PCC (0.5/1.4 $\mathrm{U} / \mathrm{mL}$ ) on MCF (A), $\alpha$-angle (B) and CT (C) of the thromboelastometry curves. Effects of addition of different amounts of PCC with or without FC $(2.4 \mathrm{~g} / \mathrm{L})$ on peak height (D), ETP (E) and lag time (F) of the thrombin generation curves. Means \pm SD $(n=3-6)$.

In thromboelastometry, effects of varying the levels of both platelets and fibrinogen were then compared. These blood components were added in various combinations to diluted plasma, while keeping the final extent of dilution constant. The three-dimensional plot of Fig. 4A indicates that, up to $250 \times 10^{9}$ platelets/L and $2.7 \mathrm{~g}$ fibrinogen/L, the MCF increased with higher levels of both platelets and fibrinogen (Fig. 4A). Even at the highest fibrinogen concentrations, the MCF raised with the platelet count. This is also apparent from a plot of the $\alpha$-angle, although this parameter reached a maximal value at lower fibrinogen and platelet levels (Fig. 4B). Regression analysis revealed that the platelet count contributed more strongly to the MCF than the fibrinogen level; with a standardized regression coefficient $\beta$ of 0.760 and 0.498 , respectively (Suppl. Table 1). 

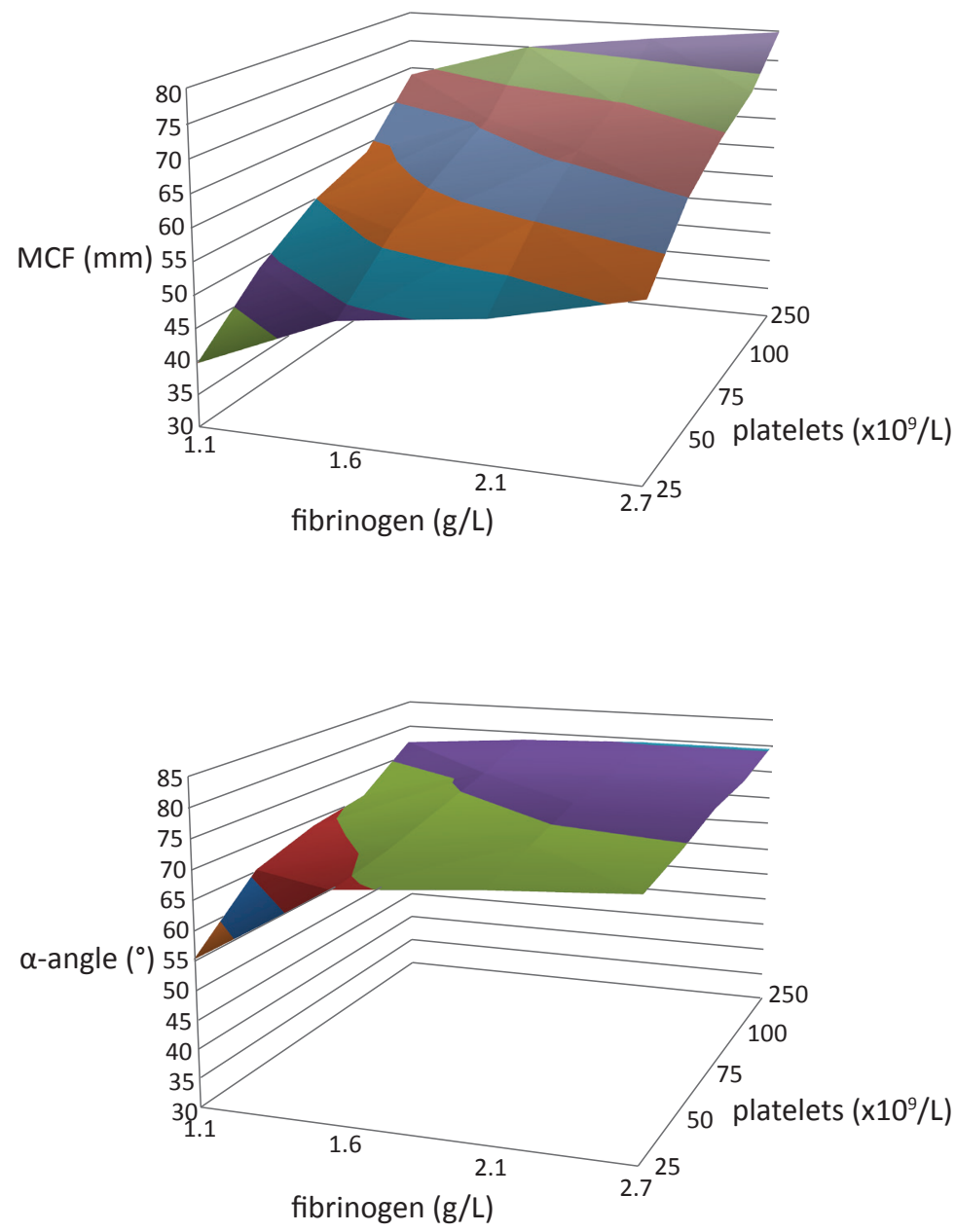

Figure 4: Combined contribution of fibrinogen and platelets to thromboelastometry. Plasma was diluted as in Fig. 3; fibrinogen levels were adjusted to $1.1-2.7 \mathrm{~g} / \mathrm{L}$, while platelets were added at $25-250 \times 10^{9} / \mathrm{L}$. Thromboelastometry curves were analyzed, as described. Graphs show effect of fibrinogen and platelet variation on MCF (A) and $\alpha$-angle (B). See also Suppl. Table 1.

Furthermore, the relative contribution of fibrinogen to MCF appeared to be independent of the platelet count, i.e. standardized regression coefficients for fibrinogen were $>0.89$. This was also true for the $\alpha$-angle. 
Predictive variables of whole blood thromboelastometry and thrombin generation in patients undergoing hemodilution during surgery

To assess the clinical relevance of these different roles of platelets and fibrinogen in thromboelastometry, blood and plasma samples were studied from patients undergoing cardiothoracic surgery and in vivo dilution by massive fluid infusion. Blood samples were analyzed from 48 patients, with mean age of 67 (range 51-75) years, of whom 33 underwent coronary artery bypass grafting and 10 replacement of the aortic or mitral valve (Suppl. Table 2). Total fluid volume transfused during the surgery procedure was $4.6 \pm 1.4 \mathrm{~L}$ (mean $\pm \mathrm{SD}$ ). A minority (12 patients) needed transfusion with $2.2 \pm 1.5$ units packed red cells. Blood samples were obtained from the patients before and after the surgical procedure. All patients received heparin $(344 \pm 77 \mathrm{mg}$ ) after collection of the first blood sample, and the heparin was antagonized with protamine $(269 \pm 51 \mathrm{mg})$ before the second blood collection. Plasma samples were checked for absence of residual heparin activity.

In the group of patients, the platelet count dropped with $45 \%$, the hematocrit level reduced with $25 \%$, and the aPTT significantly prolonged from 30.0 (median) to $35.0 \mathrm{sec}$ (Table 1). Plasma levels of prothrombin, antithrombin and factor $X$ reduced with $41-46 \%$, while fibrinogen decreased from 3.50 (median) to $1.90 \mathrm{~g} / \mathrm{L}$. In remaining plasma samples from a subgroup of 7 patients, levels of other coagulation factors were determined; factors VII, VIII and IX were reduced by $46 \%, 32 \%$ and $38 \%$, respectively. Overall, this pointed to a dilution of $\sim 40 \%$ of most blood components due to fluid infusion.

Whole blood samples pre- and post-surgery from all patients were analyzed by thromboelastometry upon triggering with tissue factor (Table 2). As expected, curves of clot formation narrowed significantly, in that the MCF decreased from median 66.0 $(62.0-70.0)$ to $55.0(48.0-60.0) \mathrm{mm}$, while the $\alpha$-angle decreased from $76.0(74.0-79.0)$

Table 1: Hematological parameters of patients before and after cardiothoracic surgery. Blood was obtained from 48 patients before and after a CPB procedure. Inactivation of heparin was checked in all post-CPB samples. Mean changes $(\Delta)$ due to the surgery procedure are indicated in percentages. Medians with interquartile ranges $(n=48)$. $* * p<0.001 v$ s. pre-surgery.

\begin{tabular}{|l|l|l|l|}
\hline Parameter & Pre-surgery & Post-surgery & $\Delta$ \\
\hline Platelets $\left(\times 10^{9} / \mathrm{L}\right)$ & $199(159-227)$ & $110(83.3-140)^{* *}$ & $-44.7 \%$ \\
\hline Hematocrit $(\mathrm{ml} / \mathrm{ml})$ & $0.32(0.30-0.36)$ & $0.24(0.23-0.26)^{* *}$ & $-25.0 \%$ \\
\hline Hemoglobin $(\mathrm{mM})$ & $6.70(6.10-7.50)$ & $5.00(4.68-5.53)^{* *}$ & $-25.4 \%$ \\
\hline aPTT (s) & $30.0(28.0-30.0)$ & $35.0(32.0-38.0)^{* *}$ & $16.7 \%$ \\
\hline Prothrombin (\%) & $103(87.0-118)$ & $55.5(43.5-64.0)^{* *}$ & $-46.1 \%$ \\
\hline Antithrombin (\%) & $98.0(90.5-109)$ & $57.5(52.0-65.8)^{* *}$ & $-41.3 \%$ \\
\hline Factor X (\%) & $86.0(74.5-109)$ & $47.5(42.3-58.0)^{* *}$ & $-44.8 \%$ \\
\hline Fibrinogen (g/L) & $3.50(2.90-4.30)$ & $1.90(1.50-2.30)^{* *}$ & $-45.7 \%$ \\
\hline
\end{tabular}


Table 2. Parameters of thromboelastometry in whole blood from patients before and after surgery. Thromboelastometry was determined in whole blood from 48 patients pre- and post-surgery, as described in the methods section. Data are medians with interquartile ranges. ${ }^{*} p<0.05,{ }^{*} p<0.001 v$ s. corresponding pre-surgery.

\begin{tabular}{|l|l|l|l|}
\hline Parameter & Pre-surgery & Post-surgery & $\Delta$ \\
\hline MCF $(\mathrm{mm})$ & $66.0(62.0-70.0)$ & $55.0(48.0-60.0)^{* *}$ & $-16.7 \%$ \\
\hline$\alpha$-angle $\left(^{\circ}\right)$ & $76.0(74.0-79.0)$ & $70.5(63.4-72.8)^{* *}$ & $-7.2 \%$ \\
\hline CT $(\mathrm{s})$ & $112(98.0-143)$ & $135(117-156)^{*}$ & $20.5 \%$ \\
\hline CFT (s) & $70.0(57.0-85.0)$ & $104(88.0-138)^{* *}$ & $48.6 \%$ \\
\hline
\end{tabular}

to 70.5 (63.4-71.80) degrees. Both the CT and clot formation time (CFT) significantly prolonged. During the 30 minutes of measurement, no signs of fibrin degradation were observed (not shown).

Multiple regression analysis demonstrated that in pre-surgery as well as in postsurgery samples, the platelet count and fibrinogen level were significant predictors of whole blood thromboelastometry. Standardized regression coefficients indicated that platelet count and fibrinogen level contributed similarly to the variation in MFC (Table 3). In contrast, variation in red blood cell count (hematocrit) or prothrombin level did not significantly add to this variation in either pre-surgery or post-surgery samples $(p>0.1)$.

Thromboelastometry and thrombin generation were also determined in plasma samples prepared from pre- and post-surgery blood (Table 4). Post-surgery thromboelastometry curves, obtained with plasma containing phospholipid vesicles, showed a consistent reduction in MCF (-46\%) and $\alpha$-angle (-11\%) and a prolongation of the CT $(+30 \%)$. These parameters also changed with platelets present. Analysis of thrombin generation measurements indicated a marked dilution effect post-surgery on thrombin peak height $(-28 \%)$ and ETP $(-7 \%)$, particularly when assessed with phospholipids. Exclusion of the data from patients, who had received red blood cells $(n=12)$, resulted in similar changes of thromboelastometry and thrombin generation data (not shown).

To determine the potential for normalization, post-surgery plasma samples were supplemented with fibrinogen concentrate and PCC. These concentrates were added at amounts corresponding to the expected effect of transfusion of 2 units fresh frozen plasma, ${ }^{5}$ i.e., $0.4 \mathrm{~g} / \mathrm{L}$ fibrinogen plus $0.11 \mathrm{U} / \mathrm{mL}$ PCC. This addition significantly increased MCF in the presence of phospholipids from 16.5 (12.5-20.0) to $21.0(17.0-25.0) \mathrm{mm}$, but left the parameter unchanged in the presence of platelets (Table 4). The same addition caused a $25-33 \%$ increase in thrombin generation (thrombin peak height and ETP), both with phospholipids and platelets. 
Table 3. Predicting variables of thromboelastometry in whole blood from patients before and after surgery. Fibrin clot formation was measured in whole blood from 48 patients pre- and post-surgery. Predicting variables of thromboelastometry MCF were obtained by linear, multiple regression analysis. Abbreviations: $b$ indicates unstandardized regression coefficient; SE $b$, standard error of $b ; \beta$, standardized regression coefficient.

\begin{tabular}{|l|l|l|l|l|l|}
\hline & b & SE b & $\beta$ & p-value & $\begin{array}{l}\text { partial } \\
\text { correlation }\end{array}$ \\
\hline Pre-surgery \\
\hline Step 1 & \multicolumn{5}{|l|}{} \\
\hline Constant & 45.59 & 3.985 & & $<0.001$ & \\
\hline Fibrinogen & 2.655 & 0.986 & 0.382 & 0.011 & 0.424 \\
\hline Platelets & 0.054 & 0.018 & 0.422 & 0.005 & 0.460 \\
\hline Step 2 & \multicolumn{5}{|l|}{} \\
\hline Constant & 44.07 & 9.627 & & $<0.001$ & \\
\hline Fibrinogen & 3.031 & 1.079 & 0.436 & 0.009 & 0.450 \\
\hline Platelets & 0.062 & 0.021 & 0.485 & 0.006 & 0.471 \\
\hline Hematocrit & 7.989 & 23.92 & 0.047 & 0.741 & 0.060 \\
\hline Prothrombin & -0.042 & 0.044 & -0.152 & 0.348 & -0.169 \\
\hline Post-surgery & \multicolumn{5}{|l|}{} \\
\hline Step 1 & \multicolumn{5}{|l|}{} \\
\hline Constant & 33.805 & 2.505 & & $<0.001$ & \\
\hline Fibrinogen & 5.783 & 1.296 & 0.507 & $<0.001$ & 0.608 \\
\hline Platelets & 0.080 & 0.021 & 0.439 & $<0.001$ & 0.552 \\
\hline Step 2 & \multicolumn{5}{|l|}{} \\
\hline Constant & 27.95 & 7.122 & & $<0.001$ & \\
\hline Fibrinogen & 5.491 & 1.502 & 0.481 & 0.001 & 0.543 \\
\hline Platelets & 0.080 & 0.025 & 0.440 & 0.003 & 0.498 \\
\hline Hematocrit & 21.06 & 25.14 & 0.084 & 0.408 & 0.147 \\
\hline Prothrombin & 0.024 & 0.073 & 0.048 & 0.749 & 0.057 \\
\hline
\end{tabular}


Table 4: Comparison of thromboelastometry and thrombin generation tests in patient plasmas before and after surgery. Plasma samples from patients, before and after surgery, were supplemented with either 4 $\mu \mathrm{M}$ phospholipid vesicles or $100 \times 10^{9}$ platelets/L, as indicated. Coagulation was triggered with $10 \mathrm{pM}$ tissue factor. Parallel plasma samples post-surgery were supplemented with fibrinogen concentrate (FC, $0.4 \mathrm{~g} / \mathrm{L}$, f.c.) and prothrombin complex concentrate (PCC, $0.11 \mathrm{U} / \mathrm{mL}$, f.c.). Medians with interquartile ranges $(n=48)$. $* p<0.05, * * p<0.001$ vs. pre-surgery samples, or vs. no addition.

\begin{tabular}{|c|c|c|c|c|}
\hline Parameter & Pre-surgery & Post-surgery & $\Delta$ & Post + FC/PCC \\
\hline \multicolumn{5}{|c|}{ Thromboelastometry } \\
\hline \multicolumn{5}{|c|}{ phospholipid vesicles } \\
\hline $\mathrm{MCF}(\mathrm{mm})$ & $30.5(26.0-37.0)$ & $16.5(12.5-20.0)^{* *}$ & $-46 \%$ & $21.0(17.0-25.0)^{* *}$ \\
\hline$\alpha$-angle $\left({ }^{\circ}\right)$ & $79.0(77.0-81.0)$ & $70.0(63.0-74.0)^{* *}$ & $-11 \%$ & $74.0(71.0-78.0)^{* *}$ \\
\hline $\mathrm{CT}(\mathrm{s})$ & $89.0(83.0-103)$ & $116(100-132)^{* *}$ & $30 \%$ & $90.5(77.0-105)^{* *}$ \\
\hline \multicolumn{5}{|l|}{ Platelets } \\
\hline $\mathrm{MCF}(\mathrm{mm})$ & $73.0(71.0-76.0)$ & $65.0(62.0-69.0)^{* *}$ & $-11 \%$ & $63.5(59.0-66.0)$ \\
\hline$\alpha$-angle $\left({ }^{\circ}\right)$ & $79.0(77.0-80.0)$ & $77.0(75.0-78.0)^{* *}$ & $-2.5 \%$ & 76.5 (74.0-79.0) \\
\hline $\mathrm{CT}(\mathrm{s})$ & $104(93.0-135)$ & $111(97.0-127)$ & $6.7 \%$ & $95.5(85.0-112)^{* *}$ \\
\hline \multicolumn{5}{|c|}{ Thrombin generation in plasma } \\
\hline \multicolumn{5}{|c|}{ phospholipid vesicles } \\
\hline Peak height (nM) & $297(249-325)$ & $213.6(179-232)^{* *}$ & $-28 \%$ & $269.5(230-295)^{* *}$ \\
\hline $\operatorname{ETP}(\mathrm{nM} \times \mathrm{min})$ & $1159(997-1313)$ & $1083(922-1252)^{* *}$ & $-6.6 \%$ & 1415 (1291-1563)** \\
\hline \multicolumn{5}{|l|}{ Platelets } \\
\hline Peak height (nM) & $144.5(114-168)$ & $133.6(113-151)^{*}$ & $-7.5 \%$ & $167.1(147-191)^{* *}$ \\
\hline $\operatorname{ETP}(\mathrm{nM} \times \mathrm{min})$ & $1282(1094-1412)$ & $1159(1041-1358)^{*}$ & $-9.6 \%$ & $1536(1423-1805)^{* *}$ \\
\hline
\end{tabular}

The MCF of whole blood thromboelastometry with pre- and post-surgery samples was dependent on the fibrinogen level (partial correlation coefficients 0.917 and 0.890 , respectively, $\mathrm{p}<0.001$ ). Notably, the MCF from curves obtained with whole blood samples correlated significantly with the MCF measured with plasma plus phospholipids $(R=0.83$, $p<0.001)$ or with plasma plus platelets $(R=0.75, p<0.001)$ (Fig. 5). On the other hand, the various parameters of thromboelastometry curves (whole blood or plasma) did not correlate with those of thrombin generation $(p>0.2)$. Together, this indicated that the predictive variables of thromboelastometry curves of patient whole blood and plasma were similar, but differed from those predicting thrombin generation curves. 
A

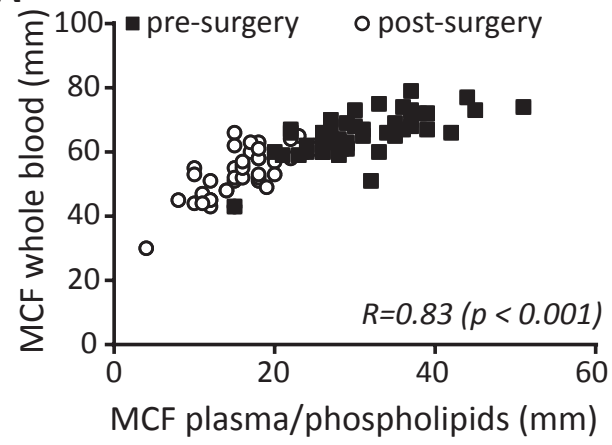

B

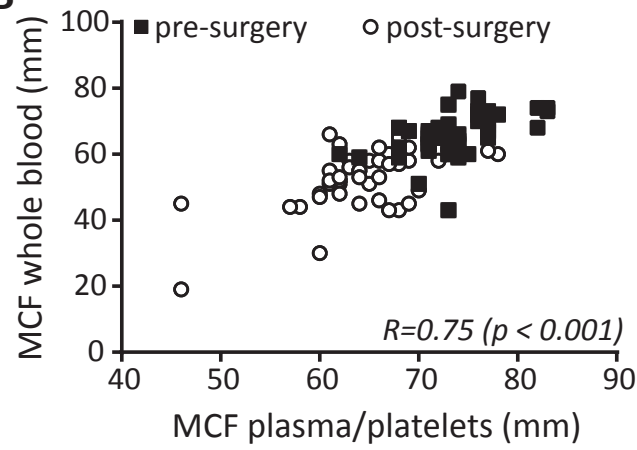

Figure 5: High correlation of thromboelastometry MCF values in whole blood and plasma samples. Thromboelastometry was measured in whole blood and plasma with phospholipids or platelets $(100 \times$ $10^{\%} / \mathrm{L}$ ) from 48 patients. MCF was derived from the thromboelastometry curves. (A) MCF in whole blood vs. plasma/phospholipids, (B) MCF in whole blood vs. plasma/platelets. Correlation coefficients and p-values are given of combined pre- and post-surgery data.

\section{Discussion}

In this paper, we studied principal sources of variation of the processes of elastic fibrin clot formation (thromboelastometry) and thrombin generation under conditions of in vitro or in vivo dilution. Coagulation in all cases was fully activated with tissue factor. $^{21}$ It appeared that, in whole blood or plasma diluted in vitro to $40 \%$, both the platelet count and fibrinogen concentration determine the kinetics and extent of fibrin clot formation in additive ways, with no more than limited contributions of red blood cells and vitamin K-dependent coagulation factors. In other words, certain (threshold) values of thromboelastometry parameters could be obtained by supplementation of platelets and fibrinogen at different relative amounts, but not by red blood cells. On the other hand, the best way to restore impaired thrombin generation in diluted plasma was by increasing the levels of vitamin K-dependent coagulation factors.

In the present paper we find that, under conditions of hemodilution, thromboelastometry parameters in whole blood are not improved by raising the hematocrit. A limited contribution of red blood cells is also apparent from the observation that curve parameters were similar in whole blood and in platelet-containing plasma (without red blood cells). Other authors, using undiluted blood have reported a negative contribution of the hematocrit on thromboelastometry parameters. ${ }^{22-24}$ Comparing with the present results, this suggests that under high viscous conditions, as in undiluted blood, red blood cells interfere with the formation of an elastic platelet-fibrin clot, while this interference becomes smaller under less viscous conditions.

The recognition that platelets and fibrinogen in additive ways contribute to thromboelastometry parameters sheds new light on our earlier conclusion ${ }^{19}$ that platelets can partly compensate for the dilutional effect on elastic clot formation. The apparently independent contribution of platelets and fibrinogen most likely reflects differences in 
function in this process, i.e. providing elasticity to the fibrin clot by contraction (platelets) and by determining the mass of a fibrin clot (fibrinogen). ${ }^{25}$ This suggestion is supported by the finding that also in blood samples from patients subjected to in vivo dilution, the platelet count and fibrinogen level are independent variables of the clot-forming process.

Marked differences were found, when comparing the effects of factor concentrates on thromboelastometry and thrombin generation under conditions of dilution in vitro or in vivo. Supplementation of fibrinogen concentrate, but not of PCC, in a dosedependent way restored elastic fibrin clot formation, as detected by an increased MCF and $\alpha$-angle, without affecting thrombin generation. Interestingly, the CT was hardly changed by PCC addition to whole blood or plasma, which agrees with published findings that the CT prolongs when coagulation factors fall below $35 \%$ of normal. ${ }^{26}$ Conversely, supplementation of PCC, but not of fibrinogen concentrate, restored thrombin generation. These data indicate that, although thromboelastometry is often viewed as an integrated global measure of coagulation, its sensitivity to reduced levels of vitamin K-dependent coagulation factor is limited.

Human and large animal studies indicate that both types of factor concentrates can improve hemostasis and reduce blood loss after major trauma. ${ }^{4,15-17}$ In porcine models, infusion of a factor concentrate, not containing anticoagulant proteins, in contrast to fibrinogen concentrate, promoted disseminated intravascular coagulation and thus caused a prothrombotic condition. ${ }^{27,28}$ In vitro analysis indeed indicates that the absence of anticoagulant factors in such concentrates promotes thrombin generation. ${ }^{29}$ In the present study, we find a prolonged CT (thromboelastometry) and lag time (thrombin generation) in the presence of a high concentration of PCC, which hence reflects the presence of anticoagulant factors that delay the formation of thrombin.

In patient plasmas after surgery, parameters of thrombin generation were less strongly reduced than those of thromboelastometry. Impairment in thrombin generation is demonstrated by the reduction in thrombin peak level as well as by the prolongation of the CT. Comparison of the curve parameters learned that the thrombin peak height was a better responsive indicator for dilution than the ETP. The latter agrees with other papers reporting on effects of in vitro dilution in thrombin generation. ${ }^{21,30}$

Taken together, we conclude that the platelet count and fibrinogen level, in additive ways, but not the amount of red blood cells, determine the extent of elastic fibrin clot formation in diluted whole blood or plasma. Thrombin generation in diluted plasma, on the contrary, is dependent on vitamin K-dependent coagulation factors. By implication, clinical decisions for transfusion with fibrinogen or plasma that are based on thromboelastometry should take into account the platelet concentration.

\section{Acknowledgments}

We thank K. Hughes for experimental support. We thank Drs. R. Nuijtinck and A. Verheijen for stimulating discussions. 
What is known on this topic

- Fibrinogen is a key determinant of elastic fibrin clot formation, assessed by wholeblood thromboelastometry or thromboelastography

What this paper adds

- Platelets and fibrinogen contribute in additive and independent ways to elastic fibrin clot formation under conditions of in vitro or in vivo dilution.

- In dilutional coagulopathy, platelets and fibrinogen, but not red blood cells or vitamin K-dependent coagulation factors, determine thromboelastometry parameters measured in whole-blood and plasma.

- Clinical decisions for transfusion of fibrinogen or plasma based on thromboelastometry should take into account the platelet concentration.

- In diluted plasma, thrombin generation is more dependent on vitamin K-dependent coagulation factors than elastic clot formation. 


\section{References}

1. Brenni M, Worn M, Bruesch M, Spahn DR, Ganter MT. Successful rotational thromboelastometryguided treatment of traumatic haemorrhage, hyperfibrinolysis and coagulopathy. Acta Anaesthesiol Scand. 2010;54(1):111-117.

2. Tirosh-Wagner $T$, Strauss $T$, Rubinshtein $M$, et al. Point of care testing in children undergoing cardiopulmonary bypass. Pediatr Blood Cancer. 2011;56(5):794-798.

3. Wegner J, Popovsky MA. Clinical utility of thromboelastography: one size does not fit all. Semin Thromb Hemost. 2010;36(7):699-706.

4. Schochl H, Nienaber U, Hofer G, et al. Goal-directed coagulation management of major trauma patients using thromboelastometry (ROTEM)-guided administration of fibrinogen concentrate and prothrombin complex concentrate. Crit Care. 2010;14(2):R55.

5. Schols SE, Lance MD, Feijge MA, et al. Impaired thrombin generation and fibrin clot formation in patients with dilutional coagulopathy during major surgery. Thromb Haemost. 2010;103(2):318-328.

6. Hemker HC, Giesen P, Al Dieri R, et al. Calibrated automated thrombin generation measurement in clotting plasma. Pathophysiol Haemost Thromb. 2003;33(1):4-15.

7. Baglin T. The measurement and application of thrombin generation. Br J Haematol. 2005;130(5):653661.

8. Schols SE, Heemskerk JW, van Pampus EC. Correction of coagulation in dilutional coagulopathy: use of kinetic and capacitive coagulation assays to improve hemostasis. Transfus Med Rev. 2010;24(1):44-52.

9. Callow CR, Swindell R, Randall W, Chopra R. The frequency of bleeding complications in patients with haematological malignancy following the introduction of a stringent prophylactic platelet transfusion policy. Br J Haematol. 2002;118(2):677-682.

10. Fenger-Eriksen C, Lindberg-Larsen $M$, Christensen $A Q$, Ingerslev J, Sorensen B. Fibrinogen concentrate substitution therapy in patients with massive haemorrhage and low plasma fibrinogen concentrations. $\mathrm{Br} \mathrm{J}$ Anaesth. 2008;101(6):769-773.

11. Thorarinsdottir HR, Sigurbjornsson FT, Hreinsson K, et al. Effects of fibrinogen concentrate administration during severe hemorrhage. Acta Anaesthesiol Scand. 2010;54(9):1077-1082.

12. Lance MD, Ninivaggi $M$, Schols SE, et al. Perioperative dilutional coagulopathy treated with fresh frozen plasma and fibrinogen concentrate: a prospective randomized intervention trial. Vox Sang. 2012;103(1):2534.

13. Demeyere R, Gillardin S, Arnout J, Strengers PF. Comparison of fresh frozen plasma and prothrombin complex concentrate for the reversal of oral anticoagulants in patients undergoing cardiopulmonary bypass surgery: a randomized study. Vox Sang. 2010;99(3):251-260.

14. Rahe-Meyer N, Sorensen B. For: Fibrinogen concentrate for management of bleeding. J Thromb Haemost. 2011;9(1):1-5.

15. Dickneite G, Doerr B, Kaspereit F. Characterization of the coagulation deficit in porcine dilutional coagulopathy and substitution with a prothrombin complex concentrate. Anesth Analg. 2008;106(4):10701077, table of contents.

16. Dickneite G, Pragst I. Prothrombin complex concentrate vs fresh frozen plasma for reversal of dilutional coagulopathy in a porcine trauma model. Br J Anaesth. 2009;102(3):345-354.

17. Fries $D$, Haas $T$, Klingler $A$, et al. Efficacy of fibrinogen and prothrombin complex concentrate used to reverse dilutional coagulopathy--a porcine model. Br J Anaesth. 2006;97(4):460-467.

18. Rosing J, Tans G, Govers-Riemslag JW, Zwaal RF, Hemker HC. The role of phospholipids and factor Va in the prothrombinase complex. J Biol Chem. 1980;255:274-283.

19. Schols SE, Feijge MA, Lance MD, et al. Effects of plasma dilution on tissue-factor-induced thrombin generation and thromboelastography: partly compensating role of platelets. Transfusion. 2008;48(11):23842394.

20. Vanschoonbeek K, Feijge MA, Van Kampen RJ, et al. Initiating and potentiating role of platelets in tissue factor-induced thrombin generation in the presence of plasma: subject-dependent variation in thrombogram characteristics. J Thromb Haemost. 2004;2(3):476-484.

21. Schols SE, van der Meijden PE, van Oerle R, et al. Increased thrombin generation and fibrinogen level after therapeutic plasma transfusion: relation to bleeding. Thromb Haemost. 2008;99(1):64-70.

22. Bochsen L, Johansson PI, Kristensen AT, Daugaard G, Ostrowski SR. The influence of platelets, plasma and 
red blood cells on functional haemostatic assays. Blood Coagul Fibrinolysis. 2011;22(3):167-175.

23. Jensen AS, Johansson PI, Idorn L, et al. The haematocrit - an important factor causing impaired haemostasis in patients with cyanotic congenital heart disease. Int J Cardiol. 2013; 167: 1317-21.

24. Nagler M, Kathriner S, Bachmann LM, Wuillemin WA. Impact of changes in haematocrit level and platelet count on thromboelastometry parameters. Thromb Res. 2013;131(3):249-253.

25. Heemskerk JW, Mattheij NJ, Cosemans JM. Platelet-based coagulation: different populations, different functions. J Thromb Haemost. 2013;11(1):2-16.

26. Weiss G, Lison S, Spannagl M, Heindl B. Expressiveness of global coagulation parameters in dilutional coagulopathy. Br J Anaesth. 2010;105(4):429-436.

27. Dickneite G, Pragst I, Joch C, Bergman GE. Animal model and clinical evidence indicating low thrombogenic potential of fibrinogen concentrate (Haemocomplettan P). Blood Coagul Fibrinolysis. 2009;20(7):535-540.

28. Grottke O, Braunschweig T, Spronk HM, et al. Increasing concentrations of prothrombin complex concentrate induce disseminated intravascular coagulation in a pig model of coagulopathy with blunt liver injury. Blood. 2011;118(7):1943-1951.

29. Grottke $O$, Rossaint R, Henskens $Y$, et al. Thrombin generation capacity of prothrombin complex concentrate in an in vitro dilutional model. PLoS One. 2013;8(5):e64100.

30. Castoldi E, Rosing J. Thrombin generation tests. Thromb Res. 2011;127 Suppl 3:S21-25. 


\section{Supplemental data}

Supplemental Table 1: Relative contribution of platelets and fibrinogen to thromboelastometry curves. PRP was reconstituted to the indicated platelet number $\left(x 10^{9} / \mathrm{L}\right)$ and the relative contribution of fibrinogen to the MCF and alpha angle was analyzed by linear regression analysis. Abbreviations: $b$, unstandardized regression coefficient, SE $b$, standard error of $b ; \beta$, standardized regression coefficient $(n=5)$.

\begin{tabular}{|c|c|c|c|c|c|}
\hline & & b & SE b & $\beta$ & p-value \\
\hline \multicolumn{6}{|l|}{$M C F$} \\
\hline \multirow[t]{2}{*}{ Plt 25} & constant & 30.82 & 1.850 & & $<0.001$ \\
\hline & fibrinogen & 9.700 & 0.943 & 0.924 & $<0.001$ \\
\hline \multirow[t]{2}{*}{ Plt 50} & constant & 39.54 & 2.087 & & $<0.001$ \\
\hline & fibrinogen & 9.663 & 1.064 & 0.906 & $<0.001$ \\
\hline \multirow[t]{2}{*}{ Plt 75} & constant & 46.16 & 1.451 & & $<0.001$ \\
\hline & fibrinogen & 9.064 & 0.739 & 0.945 & $<0.001$ \\
\hline \multirow[t]{2}{*}{ Plt 100} & constant & 50.28 & 1.608 & & $<0.001$ \\
\hline & fibrinogen & 8.839 & 0.820 & 0.931 & $<0.001$ \\
\hline \multirow[t]{2}{*}{ Plt 250} & constant & 62.84 & 1.562 & & $<0.001$ \\
\hline & fibrinogen & 6.667 & 0.796 & 0.892 & $<0.001$ \\
\hline \multicolumn{6}{|l|}{ Alpha } \\
\hline \multirow[t]{2}{*}{ Plt 25} & constant & 48.80 & 2.961 & & $<0.001$ \\
\hline & fibrinogen & 9.925 & 1.509 & 0.840 & $<0.001$ \\
\hline \multirow[t]{2}{*}{ Plt 50} & constant & 61.20 & 1.300 & & $<0.001$ \\
\hline & fibrinogen & 5.431 & 0.662 & 0.888 & $<0.001$ \\
\hline \multirow[t]{2}{*}{ Plt 75} & constant & 64.74 & 1.096 & & $<0.001$ \\
\hline & fibrinogen & 4.981 & 0.559 & 0.903 & $<0.001$ \\
\hline \multirow[t]{2}{*}{ Plt 100} & constant & 67.64 & 1.288 & & $<0.001$ \\
\hline & fibrinogen & 4.232 & 0.656 & 0.835 & $<0.001$ \\
\hline \multirow[t]{2}{*}{ Plt 250} & constant & 76.34 & 1.040 & & $<0.001$ \\
\hline & fibrinogen & 1.610 & 0.530 & 0.582 & 0.007 \\
\hline
\end{tabular}


Supplemental Table 2: Patient and surgery characteristics. Means \pm SD $(n=48)$.

\begin{tabular}{|c|c|}
\hline \multicolumn{2}{|l|}{ Characteristic } \\
\hline Male/female (n) & $32 / 16$ \\
\hline Mean age in years (range) & $67(51-75)$ \\
\hline $\begin{array}{l}\text { Medication prior to surgery }(\mathrm{n}) \\
\text { - } \quad \text { aspirin } \\
\text { - } \quad \text { coumarin } \\
\text { - } \quad \text { fraxiparine }\end{array}$ & $\begin{array}{l}28 \\
2 \\
5\end{array}$ \\
\hline $\begin{array}{l}\text { Type of surgery }(\mathrm{n}) \\
\text { - } \quad \text { coronary artery bypass graft } \\
\text { - } \quad \text { valve replacement } \\
\text { - } \quad \text { other }\end{array}$ & $\begin{array}{l}33 \\
10 \\
5\end{array}$ \\
\hline Heparin during surgery + protamine $(n)$ & 48 \\
\hline Perfusion time (min) & $91 \pm 31$ \\
\hline $\begin{array}{l}\text { Type of transfusion }(\mathrm{n}) \\
\text { - } \quad \text { red blood cells } \\
\text { - } \quad \text { fresh frozen plasma } \\
\text { - } \quad \text { fluids }\end{array}$ & $\begin{array}{l}12 \\
0 \\
48 \\
\end{array}$ \\
\hline $\begin{array}{l}\text { Transfused fluids: } \\
\text { - } \quad \text { priming fluid }(\mathrm{L}) \\
\text { - } \quad \text { cardioplegia }(\mathrm{L})(\mathrm{n}=11) \\
\text { - } \quad \text { colloids/crystaloids (L) } \\
\text { - } \quad \text { total }(\mathrm{L})\end{array}$ & $\begin{array}{l}1.4 \pm 0.4 \\
1.9 \pm 0.8 \\
1.8 \pm 0.8 \\
4.6 \pm 1.4\end{array}$ \\
\hline
\end{tabular}





\section{Chapter 8}

A synthesis approach of mouse studies to identify new genes and proteins in arterial thrombosis and bleeding

Baaten CC*, Meacham S*, de Witt SM*, Feijge MA, Adams DJ, Akkerman JW, Cosemans JM, Jupe S, Kostadima M, Mattheij NJ,

Prins $\mathrm{MH}$, Ramirez-Solis R, Soehnlein O, Weber C, White JK, Ouwehand $\mathrm{WH}^{*}$, Heemskerk JW* (*equal contribution)

Submitted 


\section{Abstract}

Current antithrombotic therapies effectively, albeit partly, reduce cardiovascular diseases by preventing arterial thrombosis and thromboembolism, but are mostly accompanied by an increased risk of bleeding. Arterial thrombosis studies using genetically modified mice have been invaluable for the identification of new molecular targets. However, because of different methodologies used, comparison of these studies and hence of the roles of different mouse genes in anti-thrombotic and hemostatic effects has been difficult. Indeed, conventional meta-analysis encountered major limitations. Therefore, we developed an alternative synthesis approach to quantitatively assess 1407 published studies of arterial thrombus formation (in vivo and in vitro), thromboembolism and tail bleeding using genetically modified mice. This resulted in a comparison of 401 genes, with 20 consistently contributing to thrombus formation without affecting hemostasis. Correlational analysis indicated high correlations between collagen-dependent thrombosis models in vivo $\left(\mathrm{FeCl}_{3}\right.$ injury or ligation/compression) and in vitro. Non-collagen-dependent models (laser or photochemical injury) linked to a lesser degree. The scores per gene were integrated into a network of protein interactions in thrombosis and hemostasis (PITH) that was validated using human gene expression databases. This revealed 2,741 new nodes connected to proteins with modifying effects on thrombus formation or bleeding, and expression along the megakaryocyte lineage. Validation with knockout mice revealed that 4/8 (Apoe, Fpr2, Ifnar1, Vps13a) new genes were modifying in thrombus formation. Thereby, PITH network: (i) revealed a high degree of homology of murine and human thrombotic processes, and (ii) identified multiple new proteins in thrombosis and hemostasis as possible targets of antithrombotic agents.

\section{Introduction}

Cardiovascular disease, due to vaso-occlusive arterial thrombosis or thromboembolism, is a leading cause of disability and death worldwide. ${ }^{1}$ Extensive research over the last decades has provided drugs that result in significant, albeit incomplete reduction in disease-related mortality and morbidity. In addition to pharmacological agents lowering plasma lipids and blood pressure, drugs that suppress blood platelet or coagulation functions are most effective in the secondary prevention of thrombotic complications. ${ }^{2-4}$ Yet, current anti-platelet and anti-coagulant drugs are partially effective, while causing serious bleeding complications in $2 \%$ or more of the treated patients. Hence, the search is continued for better targeted medication that is thrombo-protective with less bleeding (the 'holy grail of thrombosis and hemostasis'). In the past two decades, experimental thrombosis models for use with genetically modified 
mice have been developed, which have validated essentially all molecular targets for antithrombotic therapy. ${ }^{3,5,6}$ In addition, studies with genetically modified mice have confirmed the roles of most known human genes in hemostasis.

In the past two decades, hundreds of mouse studies have been performed to evaluate the consequences of genetic modification or targeted pharmacological intervention on arterial thrombosis and hemostasis. Typically, these studies use low numbers of mice, and assess arterial thrombus formation or thrombo-embolism in vivo or in vitro, using a wide range of methodologies and measurement outcomes. This variation has hampered a systematic comparison of the roles of the investigated mouse genes in these processes. The published reviews so far provide mostly qualitative descriptions of the effects of genetic modification on arterial thrombus formation or tail bleeding. $3,5,7,8$ Many of the genetic mouse models faithfully phenocopy the currently known effects of Mendelian inherited bleeding, platelet or coagulation disorders of man. ${ }^{9,10}$ Given the limited efficacy of current antithrombotic treatment, there is a need for defined, quantitative comparison of published mouse studies in order to find new genes - and hence new molecular targets - that contribute to thrombus formation with minimal hemostatic effect.

Published reports on murine arterial thrombosis have a common experimental approach, in that vascular damage is induced by activation or injury of the endothelium, frequently with exposure of the collagen-containing subendothelial matrix. ${ }^{10}$ However, per study the experimental methods can greatly differ regarding (i) the type of injured vascular bed, (ii) the type and strength of the injury trigger, (iii) the equipment and detection method employed, and (iv) the parameter used for thrombosis quantification. Hence, for systematic comparison of these studies, an analytical tool needs to be developed to scale the contribution of different mouse genes/proteins to the thrombotic process. The same applies to studies, in which microfluidics devices for whole-blood perfusion are employed as proxy tests for assessment of arterial thrombus formation. ${ }^{11-13}$ These devices are relevant from a translational point of view, since flow tests can also detect genetic or acquired deficiencies in human platelet function and sense the efficacy of antithrombotic medication. ${ }^{13-17}$ When applied to genetically modified animals, microfluidic devices have already provided detailed molecular insight into the signaling processes implicated in thrombosis and hemostasis. ${ }^{18,19}$

In the present paper, we developed and validated a synthesis scoring procedure to compare over 1407 published studies on murine arterial thrombus formation, thromboembolism and/or tail bleeding. This normalization approach gave an unprecedented insight into the strength of data regarding the roles of over 401 mouse genes, in majority regulating platelet or coagulation activation. By combining these mouse meta-data with results from genomics studies in large human populations and from patients with rare inherited bleeding disorders, we furthermore constructed a network of protein interactions in thrombosis and hemostasis, which was validated using eight new mouse strains. 


\section{Methods}

\section{Selection of papers and study definition}

A PubMed search over the years 1980-2017 (September) was performed to identify papers on mouse studies and arterial thrombus formation. Keywords were mouse $\&$ arterial \& thrombus, or mouse \& thromboembolism in different combinations. We searched for primary papers: (i) in English language; (ii) describing effects of modification of a specific mouse gene or protein on arterial thrombus formation or thromboembolism; and (iii) providing quantitative data with statistics. Excluded were: papers not indicating a well-defined genetic or pharmacological perturbation; using venous thrombosis models only; or containing no quantitative data. A second PubMed search was performed to identify papers describing quantitative effects of genetic modification on tail bleeding time (keywords: mouse, tail bleeding, tail clipping, knockout \& gene in different combinations). Non-tail bleeding times were not included. Papers of the described sets as well as published reviews were further screened for additional primary papers.

From the included papers, individual studies were defined as a coherent set of measurements of thrombus formation or thromboembolism in vivo or in vitro using a particular experimental method (vascular bed, injury trigger) and a particular animal perturbation (modified gene or target protein inhibited) in comparison to the unperturbed condition (corresponding wildtype or pharmacological control), such as defined by the authors. Papers describing multiple models to measure thrombus formation, e.g., in different vessels or in vivo and in vitro, hence, provided more than one study.

\section{Registry of perturbed genes, experimental variables and outcome data per study}

In an encompassing registry, all studies were categorized per target gene or corresponding protein, according to Mouse Genome Informatics (MGI). The recorded data from papers, including references, are given in Data File 1. Basal genetic information was taken from open access databases (www.uniprot.org and_www.informatics.jax.org): i.e. gene symbols and names; mouse genome index; chromosome location; Uniprot identification number of the corresponding human protein. Per study were listed: the genetic background; type of genetic modification; pharmacological intervention if applicable; mouse phenotype regarding altered activity of platelets, coagulation, fibrinolysis or vessel wall.

Collected experimental details on the prothrombotic intervention relied on the study class (Figure 1). Registered for class I (arterial thrombosis in vivo) were: vessel and type of injury, and dosing plus duration of the injury. Recorded for class II (systemic thromboembolism in vivo) were: type and dosing of the intervention. Recorded for class III (thrombus formation in vitro) were: thrombogenic surface, and the blood flow conditions. With respect to tail bleeding (class IV), only times of (continued) bleeding were listed. Collected was all quantitative information regarding the thrombotic or thromboembolic process at perturbed (genetic modification or pharmacological intervention) and unperturbed (wildtype or control) conditions. Further noted were relevant experimental 
Class I

In vivo arterial thrombosis

Groups 1-12

a. Time-dependent parameters

b. Mass-dependent parameters (i-iii)

c. Stability parameters
Class II

Thromboembolism

Groups 20-23

d-e. Thromboembolism parameters
Class III

In vitro thrombus formation

Groups 30-34 f. Time-dependent parameters g. Mass-dependent parameters (i-iii) h. Stability parameters

Figure 1. Flowchart for scoring and consistency analysis of parameters of arterial thrombosis and thromboembolism. Scored were studies of in vivo arterial thrombus formation (class I), thromboembolism (class II), and in vitro thrombus formation (class III) for mice with a genetic modification (GM) or pharmacological treatment, in comparison to wildtype (WT) or the control condition. Indicated are scoring procedures for the various output parameters on 3- and 5-point scales (values for WT normalized to 100\%). For detailed description of groups, see Suppl. Table 1.

variables (type of measurement, detection method, analysis procedure). Differences in mean or median values between mice with(out) perturbation were considered to be statistically significant, if indicated so by the authors.

\section{Meta-analysis}

Conventional meta-analysis was performed for six of the best studied genes, i.e., Cd36, Clec1b, F12, Fcer1g, Gp6 and Vwf (Data File 1). Therefore, only those papers were taken into account in which: (i) arterial thrombosis was studied in the carotid, mesenteric or femoral vascular beds triggered by $\mathrm{FeCl}_{3}$; (ii) outcomes were presented as time to 
vascular occlusion or time to (full) thrombus formation, (iii) data were presented as means with SE or SD (if $\mathrm{n}$ was given as a range, lowest values were taken); (iv) equal variance of groups was assumed with a correction based on effect size, if the SD of one group was missing. Meta-analyses were applied assuming a random effects model, based on the DerSimonian and Laird's methodology, according to the Nordic Cochrane Group Review Manager 5.1.20,21 Calculated per gene were: standardized mean difference plus $95 \%$ confidence interval, and heterogeneity index $I^{2}$. The full meta-analysis data are given in Data File 2.

\section{Grouping of studies and outcome parameters for synthesis approach}

Studies of class I (arterial thrombosis in vivo) were grouped according to the similarity in protocol of thrombosis induction (Suppl. Table 1). For class I, the formed groups 1-12 differed in the vascular bed and trigger of thrombosis induction. The severity of intervention (dosing or duration) was not evaluated separately, since this is often incompletely described. ${ }^{10}$ For class II, formation of groups 20-23 was based on the trigger of (provoked) systemic thromboembolism or on the presence of unprovoked thromboembolism. For class III groups 30-34 were formed, depending on the thrombogenic surface used for in vitro blood flow. Studies of class IV (bleeding) were not sub-clustered.

Quantitative data from papers were collected, as defined in Suppl. Table 1. Regarding in vivo and in vitro thrombus formation (classes I and III), measurements were registered as either time-dependent parameters (a), mass-dependent parameters (bi: extent of platelet adhesion; bii: extent of occlusion or surface-area-coverage; biii: number, size or volume of thrombi), or stability parameters (c, number or frequency of embolic events). Regarding in vivo thromboembolism (class II), data were categorized as provoked (d, percentage of survival, time to death, residual platelet count) or unprovoked (e, spontaneous thrombosis or bleeding).

\section{Comparison of thrombosis models in synthesis approach}

Per study and parameter, the published quantitative data were scored on a 3-point and 5-point scale. For the 3-point scale, reported measurements indicating a significant reduction or delay in thrombus formation (or thromboembolism) after perturbation (genetic modification or pharmacological intervention) were scored as -1 ; unchanged effects as 0 ; and significant increases or acceleration of thrombus formation as +1 . For the 5-point scale, further distinction was made between a reduced/delayed thrombus formation with $<50 \%$ (score -2) or $\geq 50 \%$ (score - 1 ); and between an increased/accelerated thrombus formation with $\leq 200 \%$ (score +1$)$ or $>200 \%$ (score +2$)$. Tail bleeding times were scored on 3-point scale only: i.e. prolonged (-1), unchanged (0), or shortened $(+1)$. Given that the majority of mouse studies were carried out at similar, small sample sizes ( $n$ = 6-12 animals), no weighting was performed when averaging study scores. Mean scores per study and gene were rounded off on 3-point or 5-point scale. All original published data and derived scored parameters are provided in Data File 1 . The overall scores per 
gene for modification in arterial thrombus formation (classes I-III) and tail bleeding (class IV) are listed in Data File 3, which also includes the reported roles per gene in platelet and/or coagulant function.

\section{Reactome pathway analysis}

Reactome (www.reactome.org/) is an open source database for the exploration and analysis of human biological pathways, based on curated literature analysis. It comprises a listing of protein-protein interactions based on biochemical reactions that are mediated by specific proteins or classes of proteins (e.g., protein tyrosine kinases or GTP-binding proteins). Standard overrepresentation analysis of proteins, corresponding to the list of modifying mouse genes, was performed per Reactome pathway using the Analyze Data tool. ${ }^{22}$ Reactome was also used for pathway-topology analysis to determine the coverage of matches per pathway for genes with positive or negative scores (giving false discovery rates). Raw Reactome analysis data are given in Data File 4.

\section{Network of protein interactions in thrombosis and hemostasis (PITH)}

Human orthologous genes were identified for the 356 murine genes, of which 288 were included as modifying in class I-III and/or class IV (Data File 5). The list of encoded proteins was supplemented by 143 additional proteins encoded by genes sampled from three databases in the following order: 72 genes underlying Mendelian disorders of thrombosis and haemostasis (https://haemgen.haem.cam.ac.uk/ thrombogenomics); 68 candidate genes inferred from a meta-analysis of genome wide association studies (GWAS) for platelet count and volume; ${ }^{23}$ and 65 genes with a regulatory element within $5 \mathrm{~Kb}$ from the transcription start site and marked by the simultaneous binding of the transcription factors FLI1, GATA1, GATA2, RUNX1 and TAL1, in accordance with recent analyses of blood progenitor cells. ${ }^{24}$ The binding sites for these transcription factors, which are all relevant and essential for megakaryopoiesis and platelet formation were defined by chromatin immunoprecipitation (ChIP), combined with massive parallel sequencing (ChIP-sequencing) in primary human megakaryocytes. ${ }^{25}$ Note that 62 genes (i.e. 493-431) of these four lists are overlapping (Data File 5). Corresponding Uniprot protein identifiers were obtained for the 384 core proteins, and these were used as primary baits to search for first order interactions using the public databases Reactome (73.1\%), IntAct (26.8\%) (www.ebi.ac.uk.intact/) and knowledge obtained from the literature (0.14\%). Networks were then constructed in Cytoscape. Attribute lists of all proteins are given in Data File 5. Also included are per protein mRNA expression data from human hematopoietic stem cells and precursor cells in the megakaryocyte lineage. ${ }^{24}$

\section{New genetically modified mice to validate network prediction}

Animal experiments were approved by the local Animal Experimental Committees in Munich and Hinxton/Cambridge. Eight strains of knockout mice, representing novel genes in the PITH network, were used to validate the prediction power of the network. Five strains came from an unbiased selection of animals available from the Wellcome Trust Sanger Institute Mouse Genetics Programme (Cambridge, United Kingdom), 
generated using targeted embryonic stem cells from the European Conditional Mouse Mutagenesis (EUCOMM) program or the Knockout Mouse Project (KOMP), ${ }^{26}$ i.e. mice with homozygous deficiency in Bnip2, Dlg4, Grm8, Ifnar1 or Vps13a with corresponding wildtype controls (tm1a/b allele on a C57BL/6N genetic background). Three strains came from breedings at the Institute for Cardiovascular Prevention in Munich (Germany) and were included because of established roles in atherosclerosis, ${ }^{27,28}$ i.e. C57BL/6 mice with homozygous deficiency in Anxa1, Apoe or Fpr2 with corresponding wildtype..$^{29}$ Mice from all strains had normal platelet counts; however, platelets from only Ifnar1-deficient mice showed a $9.4 \%$ reduction in mean volume $(P<0.01, n=5)$. No other relevant information on blood phenotype was available from Mouse Genome Informatics (www.informatics. jax.org). Flow perfusion tests with whole-blood anticoagulated with $40 \mu \mathrm{M}$ PPACK, 5 units $/ \mathrm{mL}$ heparin and 50 units $/ \mathrm{mL}$ fragmin (all final concentrations) were performed as before. ${ }^{30}$ In brief, $350 \mu \mathrm{L}$ samples were flowed over microspots coated with collagen type I at a wall shear rate of $1000 \mathrm{~s}^{-1}$ during 4 min mounted on a parallel plate perfusion chamber. Brightfield contrast microscopic images were taken using a transportable EVOS microscope, ${ }^{31}$ and analyzed for surface area coverage of multilayered platelet thrombi using the free program ImageJ. ${ }^{30}$ All image analysis was blinded for the experimental condition.

\section{Statistics and correlational analysis}

Statistics on effects of gene perturbation on thrombus formation in individual studies were taken from the original papers. For all studies published before January $1^{\text {st }}, 2017$, correlation analysis was performed to compare, per study and class, scores of time-dependent, mass-dependent and thrombus stability parameters. A non-parametric Kendall's Tau-c test was used to determine association or rank correlation. Herein, the Kendall's $\tau$ value is computed as the excess of tabled concordant over discordant pairs, divided by a term representing the geometric mean of untied pairs. It reaches from 0.0 to 1.0 for tables where all entries are on one diagonal.

A standard method to assess the consistency and strength of a list with given distribution of interval variables does not exist. To statistically evaluate gene effects in classes I-III, a one-sample t-test was used for the modifying genes (mean scores $-2,-1$, $+1,+2$ ) with $n \geq 2$. A consistency parameter $(C P)$ was defined as $1-\log (P)$, reflecting the cohesion of the pooled study scores (with $95 \%$ confidence intervals). If all studies scores were equal, nearest $P$ values were calculated. For genes with $n=1$ studies, CP was set at 1 per default. For non-modifying genes (mean score of $0, S D=0$ ), the null hypothesis was considered to be true, and a $P=1.00$ was assumed. The statistical package for the social sciences was used for all analyses (SPSS version 23, IBM Statistics). 


\section{Results}

Data collection of mouse studies reporting on genetic modification or pharmacological intervention affecting arterial thrombosis, thromboembolism or tail bleeding

A PubMed search was performed for scientific publications over the period 1980-January 2017*, resulting in 550 publications containing numeral data on effects of genetic or pharmacological perturbation on arterial thrombus formation, thromboembolism or tail bleeding in mouse. The majority of the papers concerned genetically modified mice with altered platelet or coagulant functions. The published studies were classified (Figure 1) as in vivo arterial thrombosis (class I), in vivo thromboembolism (class II), in vitro thrombus formation at arterial shear rate (class III), or tail bleeding (class IV). Manuscripts as well as published reviews were screened for further primary sources, resulting in the inclusion of another 32 publications. Individual studies were defined as effects of genetic modification (or pharmacological intervention) in a particular vascular bed. This resulted in a large database with information on 1407 different studies (see Data File 1).

\section{Limitations of conventional meta-analysis}

As a first approach to compare the outcomes of mouse thrombosis studies, a conventional meta-analysis was performed for the most consistently studied genes. Given the diversity between studies in terms of vascular bed, type of injury trigger, detection methods, and parameters measured, a standard random effects model was chosen for evaluation, according to the Cochrane group RevMan 5.1 program. Only studies were included, where arterial thrombosis was induced by $\mathrm{FeCl}_{3}$ in the carotid, mesenteric or femoral vascular beds and where time-dependent parameters were reported (see Methods). For following six genes, sufficient data could be obtained from yet a small number (n) of studies: Cd36 (4), Clec1b (3), F12 (4), Fcer1g (2), Gp6 (6) and Vwf (3). The meta-analyses (Figure 2) pointed to a significant prolongation of thrombus formation for deficiency in the genes $F 12, G p 6$ and $V w f(P=0.0008-0.003)$, while there was a tendency to prolongation for deficiency in the genes Cd36, Clec1b and Fcer1g $(P=0.05-0.08)$. Apart from the low number of studies that could be compared, a limitation of the metaanalysis approach is the large heterogeneity index $\left(1^{2}=73-88 \%\right)$, even when statistical significance is reached. Given that we considered that, using a random effects model, the high heterogeneity is unavoidable, since the animal experiments by definition are small in size, and usually vary in experimental conditions and outcome parameters. Furthermore, the heterogeneity test has low power when sample sizes are small and when few trials are included. ${ }^{32}$ Accordingly, we searched for another method to compare and normalize the effects of gene knock-outs in a much larger number of published studies.

\footnotetext{
*Data from 40 papers published after January 1, 2017 were included in the overall Data File 1 (indicated by \$ in column A) and in derived Tables, but were not used for statistical evaluation.
} 
Std. Mean Difference

$\mathrm{Cd} 36$ IV, Random, 95\% Cl

Total $(95 \% \mathrm{Cl}): 1.87[-0.01,3.76]$

Test for overall effect: $Z=1.95(P=0.05)$

Heterogeneity: $I^{2}=86 \%$

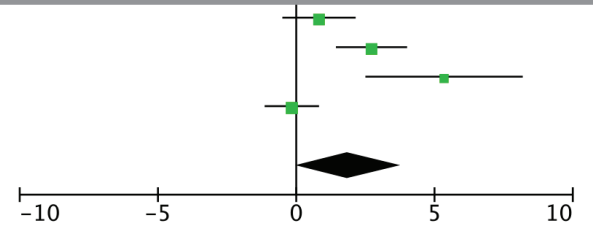

\section{Clec1b}

Total $(95 \% \mathrm{Cl}): 3.31[-0.44,7.05]$

Test for overall effect: $Z=1.73(P=0.08)$

Heterogeneity: $I^{2}=95 \%$

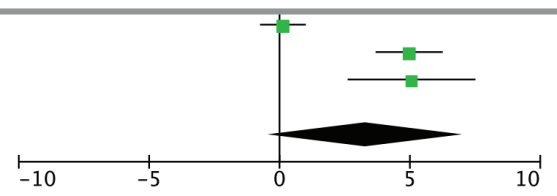

F12

Total $(95 \% \mathrm{Cl}): 4.36$ [1.57, 7.68]

Test for overall effect: $Z=2.97(P=0.003)$

Heterogeneity: $I^{2}=88 \%$

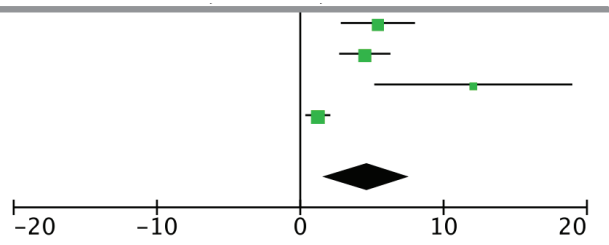

Fcer1g

Total $(95 \% \mathrm{Cl}): 0.67[-0.05,1.27]$

Test for overall effect: $Z=1.80(P=0.07)$

Heterogeneity: $I^{2}=0 \%$

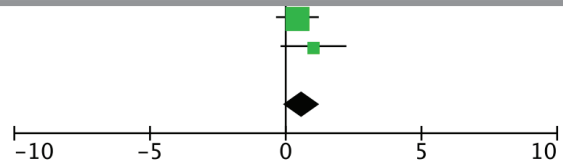

Gp6

Total $(95 \% \mathrm{Cl}): 1.16[0.37,1.96]$

Test for overall effect: $Z=2.87(P=0.003)$

Heterogeneity: $I^{2}=73 \%$

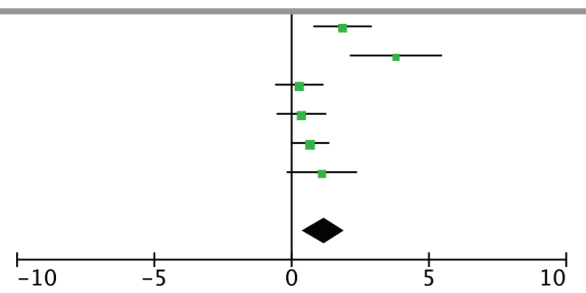

Vwf

Total (95\% Cl): 6.47 [2.70, 10.23]

Test for overall effect: $Z=3.37(P=0.0008)$

Heterogeneity: $I^{2}=77 \%$

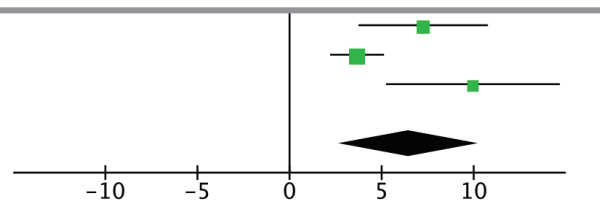

Figure 2. Meta-analysis on contribution of Cd36, Clec1b, F12, Fcer1g, GP6 and Vwf to $\mathrm{FeCl}_{3}$-induced arterial thrombosis tendency. Meta-analysis using RevMan 5.1 assuming a standard random effects model to assess overall effects of genetic deficiency in Cd36, Clec1b, F12, Fcer1g, Gp6 or Vwf on prolongation of $\mathrm{FeCl}_{3}$-induced arterial thrombus formation. Given per gene are: standardized mean difference with $95 \%$ confidence interval $(\mathrm{Cl})$, test for overall effect $(\mathrm{Z})$, and heterogeneity $\left(\mathrm{I}^{2}\right)$. For full data, see Data File 2. 
Scoring of parameters of arterial thrombus formation, thromboembolism and tail bleeding

As an alternative approach to compare and 'normalize' published gene effects on arterial thrombus formation, thromboembolism and bleeding, we aimed to develop a 'synthesis' scoring procedure that needs to be method independent. For studies on thrombus formation in vivo (class I) and in vitro (class III), we separated the published outcome data into time-, mass- or stability-dependent parameters, each of which then were scored on a 3-point and 5-point scale (Figure 1). Herein, the 3-point score discriminates between a decrease or increase of the parameter determining the thrombotic process at the perturbed condition (i.e. genetic modification or pharmacological intervention); while the 5-point score further distinguishes between a moderate/strong decrease or a moderate/strong increase. To accommodate the different measurement outcomes, we further divided the mass-dependent parameters over three sub-parameters (detailed in Suppl. Table 1).

Comparative analysis within studies of class I and class III, applied to the database as a whole, showed significant correlations between the three distinguished massdependent sub-parameters, at 3-point and mostly also at 5-point scale, with Kendall's $\tau \geq$ $0.60(p<0.050)$ (Suppl. Table 2A-B). This prompted us to combine the sub-scores into one mass-dependent score per study. Correlation analysis also pointed to a high similarity of time-, mass- and stability-dependent parameters, again within individual studies of the whole database (Suppl. Table 2C-D). In class I, these parameters correlated significantly with $p<0.001$, except when comparing time and stability parameters at a 5-point scale $(P$ $=0.210)$. In class III, with lower sample size, the time- and mass-dependent parameters correlated with $p=0.046$ (3-point) or 0.072 (5-point); thrombus stability was hardly measured in this class. Together, considering the overall high consistency of the scores for time-, mass- and stability-dependent parameters, we decided to combine these, as far as available, into one mean synthesis score (3- and 5-point scale), as a calibrated outcome of the gene modifying effect per study.

Studies to arterial thromboembolism (class II) were categorized as provoked or unprovoked, and scored on a 3- and 5-point scale. Tail bleeding times (class IV) were scored on a 3-point scale only, as these are often reported to be prolonged for an undefined time.

Comparison of study scores for different experimental models of thrombus formation and thromboembolism

In 541 cases, animals of the same breeding were used in the same paper for class I/II in vivo studies as well as for class III in vitro whole-blood studies (Data File 1, same rows). This allowed direct comparison of the scores of such studies (Suppl. Table 3). For the database as a whole, this resulted in mostly significant correlations at 3 - and 5-point scales for class I $(p<0.001, n=132)$ and class II $(p=0.006-0.165, n=14)$ studies, when 
Table 1. Correlations with scores per study in class I (in vivo thrombus formation) or class III (in vitro thrombus formation) for different vascular beds and different triggers.

\begin{tabular}{|c|c|c|c|c|c|c|}
\hline & & & 3-p & bint & 5-p & bint \\
\hline & Groups & $\mathbf{N}$ & $\begin{array}{c}\text { Kendall's } \\
\tau\end{array}$ & P-value & $\begin{array}{c}\text { Kendall's } \\
\tau\end{array}$ & P-value \\
\hline Compared to carotis & $(1-4)$ & & & & & \\
\hline Mesentary & $6-8$ & 48 & 0.79 & $<0.001$ & 0.58 & $<0.001$ \\
\hline Cremaster & $9-11$ & 23 & 0.88 & $<0.001$ & 0.60 & 0.002 \\
\hline Compared to $\mathrm{FeCl}_{3}$ & $(1,6,9)$ & & & & & \\
\hline Laser & 8,11 & 33 & 0.75 & $<0.001$ & 0.42 & 0.007 \\
\hline Photochemical & $2,7,10$ & 27 & 0.67 & $<0.001$ & 0.57 & 0.001 \\
\hline Ligation \& compression & 3,5 & 22 & 0.59 & 0.004 & 0.56 & 0.003 \\
\hline Compared to class III & (30) & & & & & \\
\hline Carotis & $1-4$ & 66 & 0.54 & $<0.001$ & 0.49 & $<0.001$ \\
\hline Mesentery & $6-8$ & 58 & 0.76 & $<0.001$ & 0.64 & $<0.001$ \\
\hline Cremaster & $9-11$ & 32 & 0.77 & $<0.001$ & 0.61 & $<0.001$ \\
\hline $\mathrm{FeCl}_{3}$ & $1,6,9$ & 86 & 0.65 & $<0.001$ & 0.59 & $<0.001$ \\
\hline Laser & 8,11 & 33 & 0.85 & $<0.001$ & 0.52 & 0.001 \\
\hline Photochemical & $2,7,10$ & 15 & 0.28 & 0.276 & 0.47 & 0.049 \\
\hline Ligation \& compression & 3,5 & 23 & 0.87 & $<0.001$ & 0.74 & $<0.001$ \\
\hline Collagen/epinephrine & 20 & 12 & 0.79 & 0.007 & 0.31 & 0.245 \\
\hline
\end{tabular}

compared to class III studies in the same paper. This indicated that, overall, study scores for in vitro thrombus formation were in good agreement with especially those for in vivo arterial thrombosis.

For a comparison of gene modification effects between different thrombosis models, studies were grouped according to the major experimental variables, notably the type of artery and the trigger to provoke injury (Figure 1; groups described in Suppl. Table 1). For class I studies, the mostly used vascular beds are carotid artery $>$ mesentery > cremaster > aorta; while the most popular injury methods are $\mathrm{FeCl}_{3}>$ laser $>$ photochemical $>$ vessel ligation or compression > electrolytic injury (Suppl. Figure $1 \mathrm{~A})$. For class II, the majority of studies apply injection of collagen/epinephrine, with fewer studies using tissue factor or reporting unprovoked thrombosis (Suppl. Figure 1B). Concerning class III, the majority of studies use collagen type-I as a thrombogenic surface (Suppl. Figure 1C). Class IV bleeding time studies were not sub-divided. 
Numbers of studies per group (type of artery and injury mode) were too low for regression analysis per gene (Suppl. Table 4). Therefore, relevant groups were combined to compare either different vascular beds or different injury modes. Markedly, the scores for carotis, mesentery or cremaster injury, averaged per mouse gene, correlated significantly at 3-point and 5-point scales (Table 1). Furthermore, in class I, average scores per mouse gene for carotis and mesentery injury (i.e., both collagen-dependent thrombosis models) correlated with high significance $(p<0.001)$ with the class III study scores (i.e., thrombus formation on collagen in vitro). Interestingly, scores from studies using the cremaster (with frequent laser injury, and less collagen-dependent) also correlated with class III study scores ( $p=0.002,5$-point).

Concerning the injury mode, studies scores for $\mathrm{FeCl}_{3}$ correlated well $(p \leq 0.008$, 3- and 5-point) with those of laser, photochemical or ligation/compression injury (Table 1). Class III scores of collagen-dependent thrombus formation in vitro correlated well on a 3-point $(p=0.007, \mathrm{n}=13)$ but not 5 -point $(p=0.245)$ scale with class II scores (thromboembolism with frequent collagen/epinephrine injection). Taken together, this analysis revealed that, in general, for this cohort of published mouse studies, effects

A

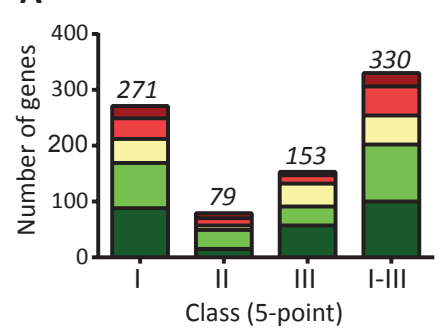

B

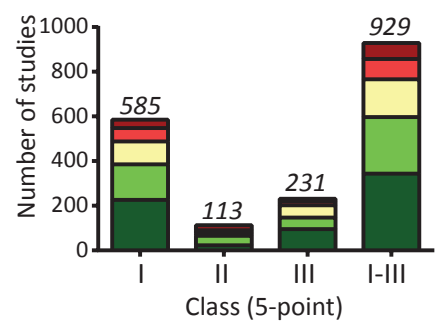

C

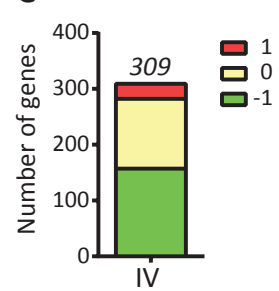

Class (3-point)

D
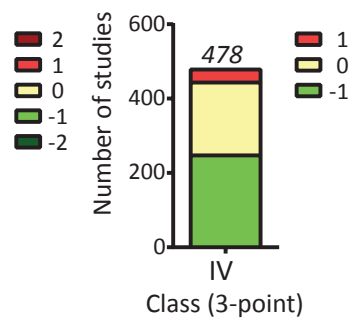

Class (3-point)
E

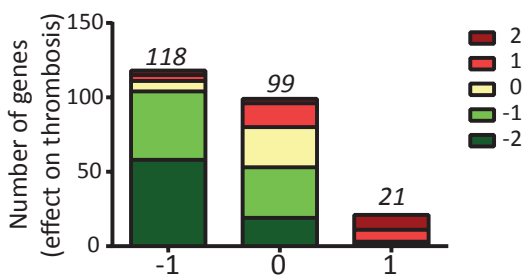

Tail bleeding (class IV)

Figure 3. Score distribution pattern of total of analyzed studies. Indicated are numbers of scored mouse genes (A, C) and studies (B, D) for classes I-III (arterial thrombosis) and class IV (tail bleeding). Scoring was on 5-point (A, B) or 3-point scale (C, D). All studies included, except for those with mice with non-comparable genetics (for example, gain-of-function mutations). E, Total numbers of genes with altered arterial thrombus formation or thromboembolism (5-point scale, classes I-III), in combination with prolonged (-1), unchanged (0) or shortened $(+1)$ tail bleeding time (class IV). 
of a gene modification were similar for the different (collagen-dependent) models, irrespective of the arterial vascular bed, injury mode, injection of collagen or in vitro whole-blood testing.

Synthesis approach of study scores to assess gene perturbation effects on arterial thrombus formation and/or hemostasis

Considering that the 5-point normalization scores are more discriminative than the 3-point scores, we used the former for further analyses of all 1407 studies. At first, mean scores were calculated per mouse gene and study class for thrombus formation/ thromboembolism. For in total 401 genes, this resulted in scored information on 271, 79 and 153 genes for classes I, II and III, respectively (Figure 3A-B). For bleeding times (class IV), scored information was obtained for 309 genes (Figure 3C-D).

Data File 3 provides a complete list with mean scores per gene of classes I-IV, and additional information on the type of genetic modification and the effects on platelet and coagulant functions. Clinically of high interest are those gene deficiencies that lead to reduced arterial thrombosis (negative class I-III scores) without increased bleeding. First analysis using all scores per gene reveals that 53 mouse genes fall into this category

A
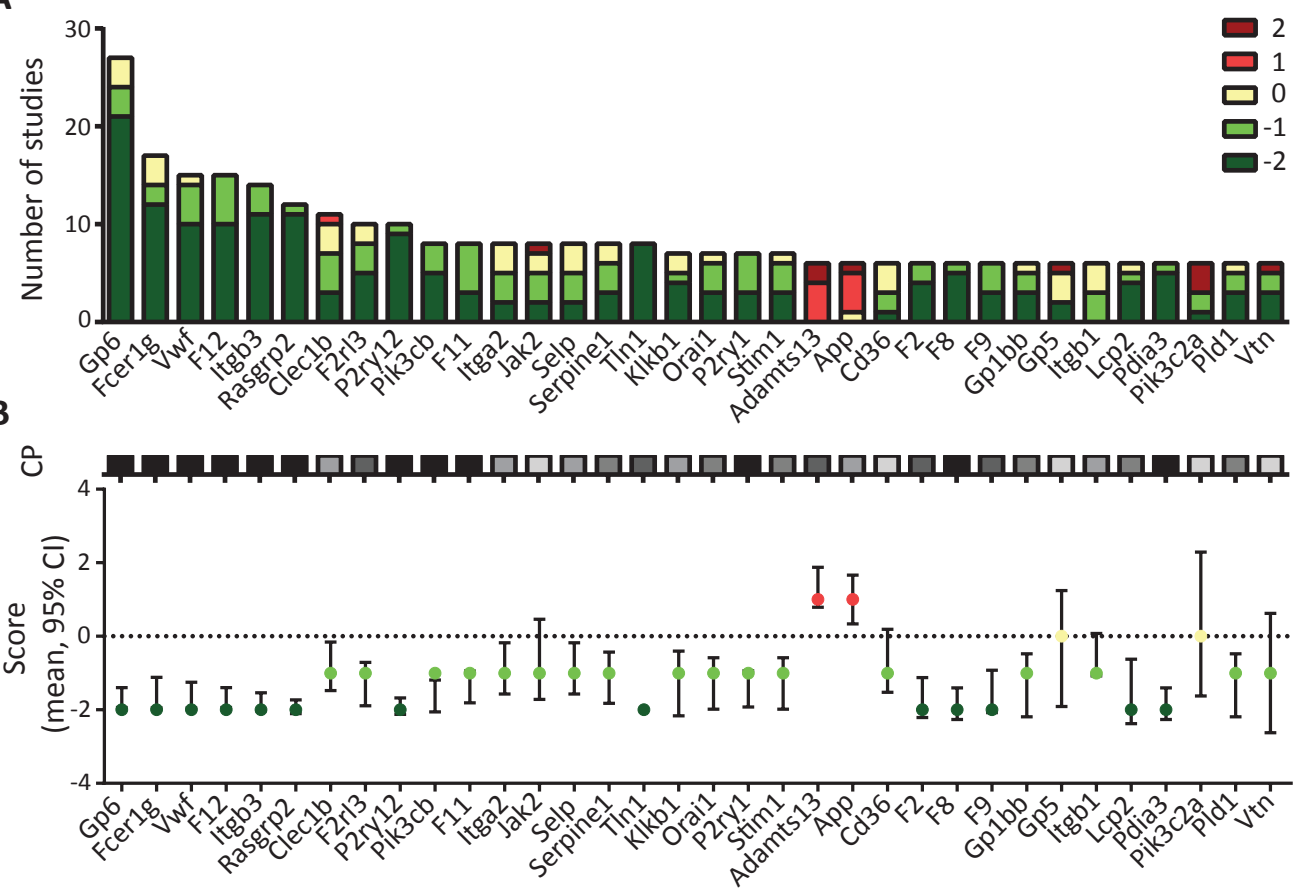

Figure 4. Study score analysis for 34 of most frequently studied genes in classes I-III for effects on arterial thrombosis tendency. A, Summation of scores per study for indicated genes and classes I-III. Data obtained from 299 studies (65.3\% collagen-dependent models; 14.8\% laser models, 6.6\% photochemical injury). B, Calculated consistency parameters (CP) per gene [1 - log $(P)]$ (scaled gray levels from 0 to 5 ), and mean scores per gene with $95 \%$ confidence intervals $(95 \% \mathrm{CI})$. 
(Figure 3E). Deficiency in 18 out of 21 genes causing a shortened tail bleeding time (anti-hemostatic effect) resulted in positive class I-III scores, suggesting a prothrombotic propensity. As expected, a -2 score was more frequently (75\%) accompanied by prolonged bleeding than a -1 score $(57 \%)$. This underlines the premise that a more severe reduction in arterial thrombus formation is more likely to be accompanied by a bleeding phenotype.

Subsequently, for the 34 best-investigated genes ( $n \geq 6$ studies in classes I-III, $65.3 \%$ collagen-dependent models), we in evaluated the distributions of individual study scores in more detail (Figure 4A). When considering the scores from individual studies as discretized ordinal variables with defined ranking, these can be treated as interval variables. This allows a calculation of $95 \%$ confidence intervals and $P$ values, whilst retaining the rounded off mean score per gene. In addition, we defined a consistency parameter $(C P)$ as $1-\log (P)$, reflecting the consistency and strength of the pooled study scores. Following this synthesis approach it appeared that, for the 34 most frequently studied genes, those with a -2 mean score showed CP values decreasing in the order of

Table 2. Mouse genes and orthologous human genes predicted to contribute to arterial thrombosis with(out) altered bleeding. Genes were selected with scores from $n \geq 3$ studies, and a CP value $>2.30$ ( $P<$ $0.05)$. Selected genes were classified according to described phenotypic changes in mice. Bold: corresponding protein targeted in current use or clinical trials.

\begin{tabular}{|c|c|c|}
\hline Protein function & Mouse gene & Human orthologue \\
\hline \multicolumn{3}{|c|}{ Antithrombotic, no prolonged bleeding } \\
\hline Plasma coagulation & $F 11, F 12$ & $F 11, F 12$ \\
\hline Plasma fibrinolysis & Serpine1 & SERPINE1 \\
\hline Platelet membrane & $\begin{array}{l}\text { Cd36, Clec1b, Dusp3, Gp6, } \\
\text { Itga2, Itga6, P2rx1, Selp }\end{array}$ & $\begin{array}{l}\text { CD36, CLEC1B, DUSP3, GP6, } \\
\text { ITGA2, ITGA6, P2RX1, SELP }\end{array}$ \\
\hline Platelet signaling & $\begin{array}{l}\text { Pik3c3, Pik3cg, Pld1, Ppia, } \\
\text { Prkca, Rhog, S100a9, Sgk1 }\end{array}$ & $\begin{array}{l}\text { PIK3C3, PIK3CG, PLD1, PPIA, } \\
\text { PRKCA, RHOG, S100A9, SGK1 }\end{array}$ \\
\hline \multicolumn{3}{|c|}{ Antithrombotic, prolonged bleeding } \\
\hline Plasma coagulation & $F 2, F 8, F 9, K l k b 1, V w f$ & $\mathbf{F 2}, F 8, F 9, K L K B 1$, VWF \\
\hline Platelet membrane & $\begin{array}{l}\text { Ano6, Cd151, F2rl3, Fcer1g, } \\
\text { Fermt3, Gna13, Gnaq, Gp1ba, } \\
\text { Gp1bb, Itgb3, Mertk, Orai1, } \\
\text { P2ry1, P2ry12, Pdia3, Pdia4, } \\
\text { Ptprj, Stim1 }\end{array}$ & $\begin{array}{l}\text { ANO6, CD151, F2RL3, } \\
\text { FCER1G, FERMT3, DNA13, } \\
\text { GNAQ, GP1BA, GP1BB, } \\
\frac{\text { ITGB3, MERTK, ORAI1, P2RY1, }}{\text { P2RY12, PDIA3, PDIA4, PTPRJ, }} \\
\text { STIM1 }\end{array}$ \\
\hline Platelet signaling & $\begin{array}{l}\text { Map3k5, Mapk8, Pdpk1, } \\
\text { Pik3cb, Plcg2, Rac1, Rasgrp2, } \\
\text { Tln1 }\end{array}$ & $\begin{array}{l}\text { MAP3K5, MAPK8, PDPK1, PIK- } \\
\text { 3CB, PLCG2, RAC1, RASGRP2, } \\
\text { TLN1 }\end{array}$ \\
\hline Platelet secretion & Nbeal2, Kcnip3, Mmp2 & NBEAL2, KCNIP3, MMP2 \\
\hline Vessel wall & Bambi & $B A M B I, B D K R B 2$ \\
\hline \multicolumn{3}{|c|}{ Not antithrombotic, prolonged bleeding } \\
\hline Vessel wall & Ceacam $1 / 2$ & CEACAM1/2 \\
\hline
\end{tabular}


Fcer1g, Gp6, F12, F8, F9, Itgb3, P2ry1, P2ry12, Pdia3, Rasgrp2, Vwf (CP 5.0) > F2, TIn1 $>$ Lcp2 > Vtn (Figure 4B). For genes with a -1 mean score, CP values decreased in the order of F11, Pik3cb > F2rl3 (CP 5.0) > Orai1, Stim1 > Gp1bb, Pld1, Serpine1 > Clec1b, Itga2, Klkb1, Selp > Itgb1, Cd36 (CP 2.0). Regarding the 'prothrombotic' genes (mean score +1$)$ Adamts 13 and App, CP values ranged between 2.9 and 4.0. In other words, the combination of mean score with calculated $\mathrm{CP}$ value (with $\mathrm{CP}>2.30$ indicating a $p$ $<0.05)$ provides a relevant indication of the strength of the literature data on the antior prothrombotic propensity of a particular mouse gene. A full overview of calculated scores and CP values per gene is given in Data File 3.

The synthesis approach further allows identifying those mouse genes with negative class I-III scores and a prolonged or unaltered bleeding tendency (-1 or 0 in class IV, respectively). Table 2 provides an overview of these genes, taking an arbitrary cut-off of $n \geq 3$ study scores available. Markedly, for most of the human orthologues in the second list, genetic deficiencies have been described that associate with impaired hemostasis.

A

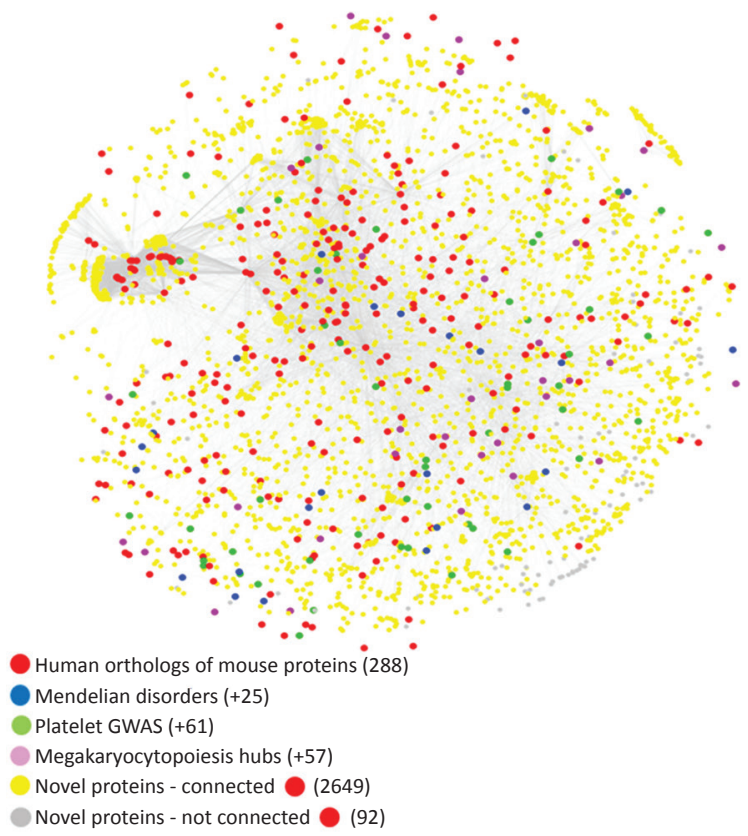

B

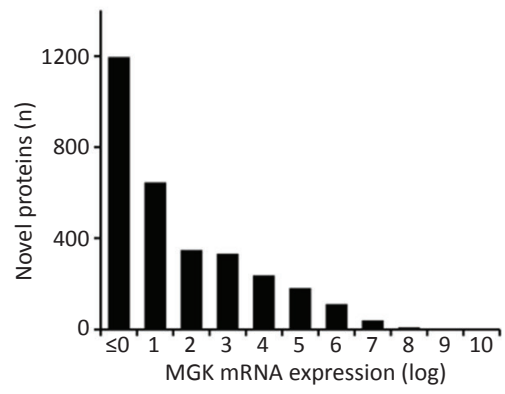

C

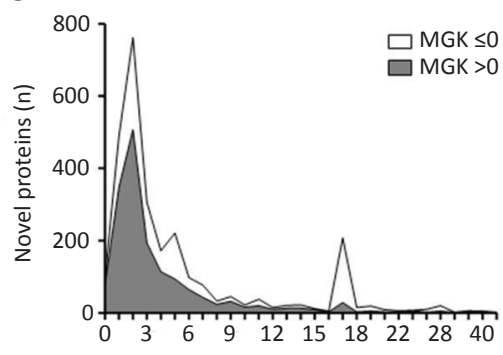

Figure 5. Network of protein interactions in thrombosis and hemostasis (PITH). A, Network formed from 431 bait proteins (nodes) and 18,452 interactions (edges). Bait nodes are color-coded: orthologues of modifying mouse proteins (red); proteins linked to rare Mendelian disorders of human platelet or coagulant function (blue); proteins linked to high association genes from genome-wide association studies (GWAS) of platelet count and volume (green); identified hubs of megakaryopoiesis (purple). Novel proteins $(2,741)$ obtained in Cytoscape by connecting the bait proteins are colored yellow, if connected to one or more modifying mouse protein (candidate proteins, 2,649), or otherwise colored grey (92). B, Distribution of 2,741 novel proteins with respect to mRNA expression in megakaryocytes (MGK). Values are given as log2 (FPKM+1) (FPKM, fragments per kilobase of transcript per million mapped reads; values $<1$ are considered as a lack of transcription). C, Distribution of 2,741 novel proteins with respect to connections (edges) with modifying mouse proteins (thrombosis and/or bleeding). 
These include genes encoding for plasma factors (coagulation, fibrinolysis), platelet proteins (membrane, signaling, secretion), or vessel wall components. Furthermore, antagonists for several human orthologous proteins are in current clinical use (thrombin inhibitors, F2; integrin $\alpha_{11 b} \beta_{3}$ antagonists, ITGB3; P2Y ${ }_{12}$ antagonists, P2RY12); or undergo investigation in clinical trials (factor XI, XII inhibitors, F11, F12; glycoprotein VI inhibitor, GP6; glycoprotein Ib-VWF inhibitors, GPIBA, VWF; phosphoinositide 3-kinase- $\beta$ inhibitor, PIK3CB).

\section{Network prediction of new genes and proteins implicated in arterial thrombus formation}

For translation of the scoring per mouse gene/protein to human pathophysiology, we performed an over-representation analysis using the Reactome database (Data File 4). The Reactome is a curated knowledgebase of biological pathways, elaborated for human megakaryocytes and platelets and for the human coagulation system. ${ }^{33}$ Enrichment analysis for 288 of the human homologues, corresponding to the modifying mouse genes (class I-III or class IV), indicated that a large part of the hemostatic system was covered (108/515 proteins). Well-covered pathways included 'Platelet activation, signaling and aggregation' (71/228), and several platelet sub-pathways; further 'Innate immune system' (53/951), 'Formation of fibrin clot' (25/39), and 'Cell surface interactions at the vascular wall' $(28 / 101)$ as well as sub-pathways of these (Data File 4). Reactome sub-analysis of human proteins corresponding to mice with negative scores for arterial thrombosis (classes I-III) or bleeding (class IV), indicated no major differences, except for a higher coverage in the former category of the pathway '(Innate) immune system'.

To develop a comprehensive system, a Cytoscape network was constructed with human protein orthologues of 288 modifying mouse genes, supplemented with 143 different human core proteins, representing major known molecular pathways in thrombosis and hemostasis (Data File 5), which were used as primary baits to retrieve 2,741 first order interactors (nodes) and 18,452 interactions (edges), primarily from the Reactome $(73.1 \%)$ and IntAct $(26.8 \%)$ databases with the remainder $(0.14 \%)$ obtained from the literature (Figure $5 \mathrm{~A}$ ). The advantage of such a network with both general and specific core types of reactions is that it results in nodes with multiple edges, hence increasing the likeness to identify physical protein-protein interactions. The expanded network of protein interactions in thrombosis and hemostasis (PITH), should facilitate the identification of new biological pathways and relevant (patho)physiological mechanisms of action. Markedly, alternative construction of the network, by first adding the human core proteins and second supplementing the mouse orthologues, resulted in $>75 \%$ of the nodes (proteins) and edges (reactions) appearing at the last step (Suppl. Figure 3). This indicates that the major part of the network is shaped by the scored mouse studies. On the other hand, the gene modifications in the scored mice correspond to essentially all human platelet and coagulation genes/proteins that are known to be linked to hemostatic insufficiencies.

Systematic analysis of the constructed network was performed for a prediction of new genes contributing to arterial thrombosis and bleeding. To identify platelet proteins, for all nodes we compared the mRNA expression levels in human hematopoietic stem cells and cells differentiating along the megakaryocyte lineage (Data File 5). For the novel 


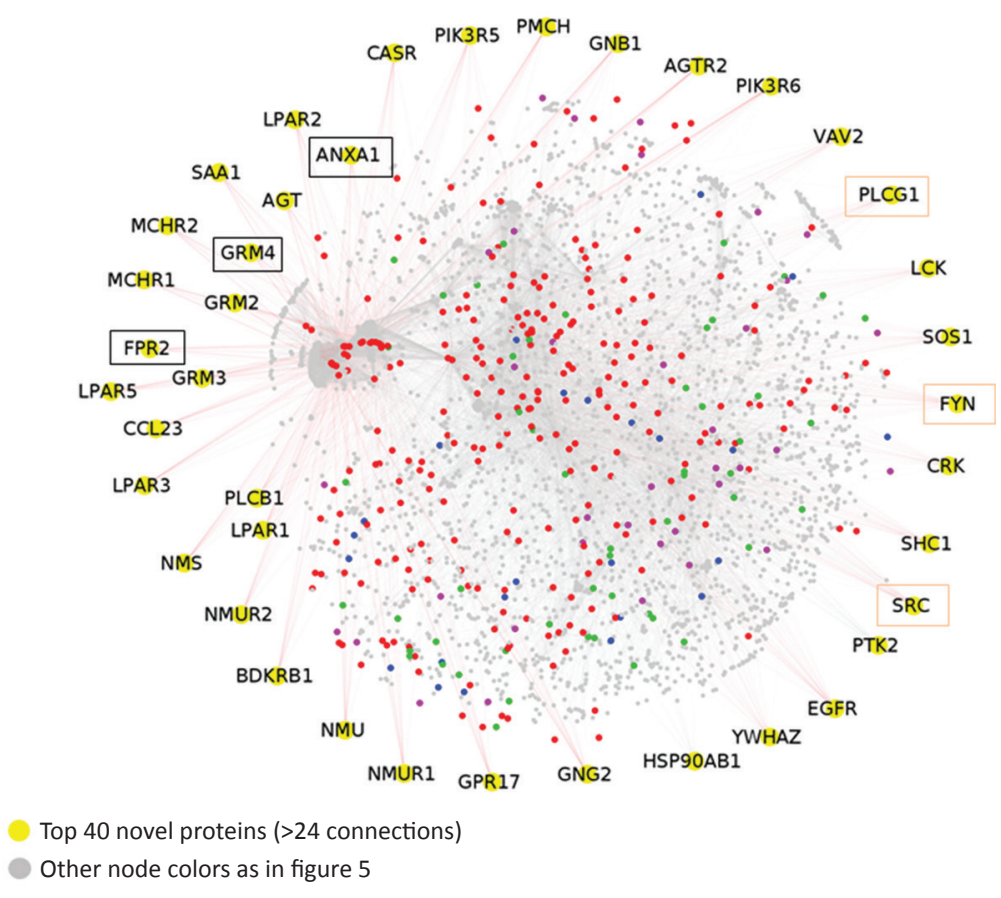

Figure 6. PITH network emphasizing top $\mathbf{4 0}$ novel proteins ( $>24$ connections). Boxed in black are orthologue mouse genes validated in this paper; boxed in orange are mouse genes without modifying effect on thrombosis or bleeding. For a complete attribute list of all genes/proteins, see Data File 5 . The full network, containing gene expression levels in human megakaryocytes ${ }^{24}$ and other annotation features, is available in Cytoscape format for download.

nodes, it appeared that the majority of the corresponding genes were transcribed in megakaryocytes (Figure 5B), and that most of these had 1-4 connections with one or more modifying proteins in the network (Figure 5C). Depiction of the top 40 of novel proteins with highest numbers of connections (Figure 6) revealed several interesting protein kinases (EGFR, FYN, LCK, PTK2) and interactors of these (CRK, SHC1, SOS1, VAV2). Interestingly, three of the highly connected 'novel' proteins (FYN, SRC, PLCG1) were already present in the mouse list, but had been scored here as non-modifying and hence were not included in the bait protein set (Figure 5C).

\section{Validation of protein interaction network using new genetically modified mice}

To validate the prediction power of the PITH network in the discovery of new genes contributing to arterial thrombosis and/or bleeding, we screened eight novel strains of knockout mice and scored these for arterial thrombus formation in vitro (class III). The animals came from two different sites with large facilities and breeding programs, i.e. the Wellcome Trust Sanger Institute Mouse Genetics Project in Cambridge (United Kingdom) and the Institute for Cardiovascular Prevention in Munich (Germany). The strains were selected from the appearance of the gene in the PITH network and the simultaneous availability of mice. First, we screened mice with a homozygous deficiency 
in Bnip2 (protein regulating cell death), Dlg4 (a membrane-associated guanylate kinase that can cluster signaling proteins), Grm8 (metabotropic glutamate receptor 8, coupled to Gi proteins), Ifnar1 (interferon receptor signaling via JAK/STAT pathway) or Vps13a (implicated in protein cycling through the trans-Golgi network) (Figure 7A). Deficiency in two out of three genes with normally high mRNA expression in megakaryocytes (Figure 7B), i.e. Ifnar1 and Vps13a (but not Bnip2), resulted in a significant reduction in thrombus formation in vitro (score -2). Deficiency in the two other genes with minor mRNA expression in megakaryocytes, Dlg4 and Grm8, did not modify thrombus formation (score 0). Support for these novel findings comes from recent papers that interferon I (ligand of Ifnar1 transcript) can regulate platelet count and function; ${ }^{34}$ and that gene defects in VPS13A are associated with chorea-acanthocytosis, with platelets from such patients showing an impairment of granule secretion. ${ }^{35}$

Furthermore, we screened mice with homozygous deficiencies in Apoe (apolipoprotein $\mathrm{E}$, controlling the catabolism of lipoprotein triglycerides; not yet evaluated in class III), Fpr2 (Gi protein-coupled formyl peptide receptor 2), or Anxa1 (recognized as ligand of FPR2) (Figure 7C). These genes have all been implicated in murine atherosclerosis, might act by blood plasma-based changes in thrombus formation, and appear with multiple edges in the PITH network (Figure 7D). Transcripts of these genes are expressed at medium to high levels in the liver, ${ }^{36}$ while only annexin A1 is expressed in megakaryocytes and neutrophils. Interestingly, of these three genes (Figure 7D), deficiencies in Apoe and Fpr 2 but not Anxa1 positively modified thrombus formation

A

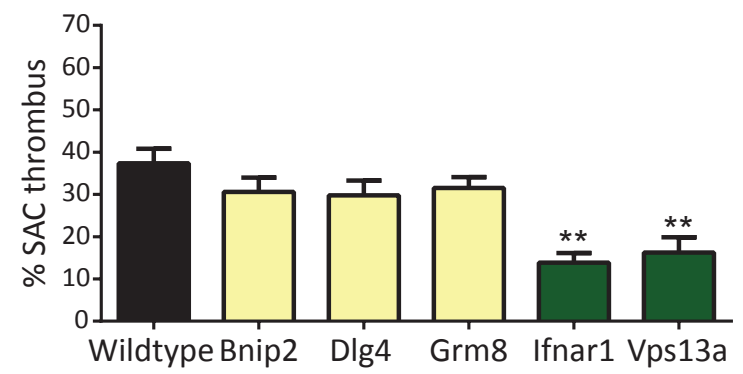

B

Connectors

MGK mRNA

\begin{tabular}{|ccccc|}
\hline Bnip2 & Dlg4 & Grm8 & Ifnar1 & Vps13a \\
\hline 2 & 7 & 25 & 3 & 2 \\
3.63 & -2.57 & -2.10 & 3.11 & 2.50 \\
\hline
\end{tabular}

\section{C}

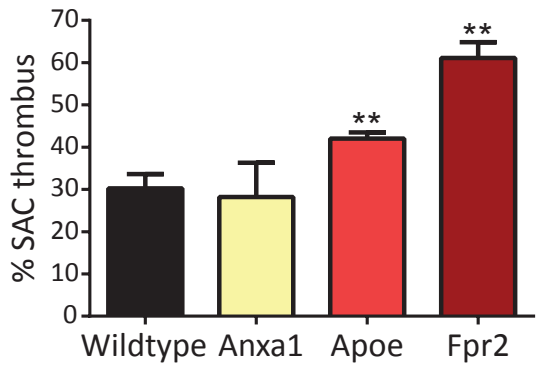

D

\begin{tabular}{|ccc|}
\hline Anxa1 & Apoe & Fpr2 \\
\hline 30 & 43 & 27 \\
1.82 & -3.40 & -1.75 \\
\hline
\end{tabular}

Figure 7. Validation of network prediction for eight novel mouse genes not examined before. Mice homozygously deficient in indicated genes were tested for arterial thrombus formation in vitro (class III). Data represent surface area coverage (SAC) of platelet thrombi on collagen. A-B, Testing of 5 genes/proteins from PITH network with suspected platelet loss-of-function defect. Mean \pm SE ( $n=6-17), * * P<0.01$. C-D, Testing of 3 genes/proteins from PITH with suspected gain-of-function defect related to atherothrombosis. Mean \pm SE $(n=6-18), * * P<0.01$. B, D, Connecting edges of human orthologues in PITH network, and relative mRNA expression in megakaryocytes (log scale). 
$\left(\right.$ Apoe $^{-\%}$, score +1 and $\mathrm{Fpr}^{-\%}$ score +2$)$. While annexin A1 is an established ligand of FPR2, blocking arterial myeloid cell adhesion and recruitment to atherosclerotic lesions, ${ }^{29}$ the present results suggest that the prothrombotic role of FPR2 on platelets is distinct from the role in leukocytes. Taken together, it appeared that 4 out of 8 novel mouse genes with a predicted contribution to arterial thrombus formation, indeed showed a phenotype.

\section{Discussion}

Murine experimental arterial thrombosis studies, mostly performed in a translational research context with mice deficient in platelet or coagulation proteins, have in common several features, such as genetic homogeneity, a small sample size, a controlled way of vascular injury, and the ability of in vivo monitoring of the thrombotic or thromboembolic process. On the other hand, although some thrombosis induction models are more commonly used than others, there is no standard approach, in that the favorite target vessel, the mode of injury as well as the recording of output parameters can differ from laboratory to laboratory and from one mouse strain to another. ${ }^{10}$ In the present synthesis paper, encompassing 1407 studies, we show for the first time that - in spite of all these experimental differences - uniform scoring of the effect of genetic modification on the thrombotic process is feasible, and that this allows direct comparison of different experimental models and different genetically modified mice. In other words, the overall agreement of scoring scales between different injury models indicates that, overall, the effect of gene modification is larger than the variation caused by differences in methodology or measurement parameters. Thus, given the similarity in scorings per gene, this systematic analysis points to a grossly comparable molecular regulation of the thrombus-forming process in different arterial beds, and even systemic (collagen-induced) thromboembolism.

The scoring on a 5-point scale, in comparison to the 3-point score, distinguishes between moderate and strong changes in thrombus formation. It reveals subtle differences between established collagen-dependent models (i.e., $\mathrm{FeCl}_{3}$ or ligation/compression injury) and less collagen-dependent thrombosis models (laser or photochemical injury). The latter models are considered to be more dependent on tissue factor activity and/ or mild endothelial damage, but less on collagen exposure, although this may depend on the severity of vascular trauma. ${ }^{37-39}$ Regression analysis indeed resulted in lower significance levels when comparing high vs. low collagen-dependent models. In human cardiovascular disease, the limited available evidence suggests that atherothrombosis is a collagen-dependent process, given the prothrombotic role of the collagen exposed on ruptured atherosclerotic plaques. ${ }^{40,41}$

For comparison of the roles in arterial thrombosis and hemostasis of multiple genes, we developed a synthesis approach taking into account the mean study scores (treated as interval variables to allow statistical comparison) and a consistency parameter 
$\mathrm{CP}$, reflecting the strength and consistency of the published data. This approach provides valuable lists of known and new possible targets for treatment of human arteriothrombosis with global indications of the bleeding risk (Table 2).

Given that only few drugs are currently available for thrombosis treatment in cardiovascular disease, the present network reveals a wealth of novel candidate target proteins with a possible or likely role in arterial thrombosis with or without bleeding. These include multiple proteins that modulate functions of platelets, blood lipids, coagulation or fibrinolysis, as well as proteins regulating vascular functions. So far, we identified in mice 19 genes that are reported to affect arterial thrombus formation and thromboembolism without effect on bleeding. The protein network analysis revealed many other novel proteins (expressed in megakaryocytes and platelets) linked to this core set, which may modulate the thrombosis and hemostasis processes in a positive or negative way.

An important finding from the current synthesis is that, in general, measurement of thrombus formation using flow chambers in vitro can serve as a proxy test for assessment of (collagen-dependent) thrombus formation in vivo. This may support implementation of 3Rs alternatives by: (i) refining in vitro procedures limiting the need for encumbering in vivo experimental research; (ii) reducing number of animals, given the relatively small volumes of blood needed for in vitro tests with microfluidic chambers, and (iii) ultimately replacement of animal by human blood.

The established network of protein interactions in thrombosis and hemostasis (PITH) reveals a wealth of candidate genes and proteins - mostly but not exclusively expressed in megakaryocytes and by implication platelets - with a high likelihood to regulate arterial thrombus formation with or without bleeding. This network can be used for testing mouse knockout models and for prioritizing genes in the gene discovery sequencing efforts for patients with rare inherited bleeding, platelet, or thrombotic disorders of unknown molecular etiology. The network furthermore provides an overall indication of the similarity of the molecular regulation of the thrombotic processes in mouse and man, since essentially all mice with deficiencies corresponding to genetic defects of human bleeding disorders scored negatively, and since both the bait and harvest proteins (genes) covered relevant pathways of the human-based Reactome database. In extensive network validation studies, we found that 4 out of 8 new mouse strains, with deficiency in a potentially modifying gene, showed an altered thrombus formation on collagen.

In summary, this paper demonstrates that a novel synthesis approach based on scoring of time-, mass- and stability-dependent parameters of thrombus formation can integrate the effects of multiple small-size mouse studies to: (i) establish a high degree of homology of murine and human thrombotic processes, and (ii) identify new molecular targets for antithrombotic therapy. 


\section{Key points}

- A new synthesis method compares the roles of 401 genes in arterial thrombosis, with 19 contributing without affecting hemostasis.

- Based on this synthesis, a network of protein interactions in thrombosis and hemostasis (PITH) was developed and validated for 8 novel genes.

\section{Acknowledgements}

SdW, JMC and JWH have received funding from the Center for Translational Molecular Medicine (INCOAG), the Netherlands Heart Foundation (2011T6), the Cardiovascular Center Maastricht and ZonMW (MKMD 114021004). Research in the Ouwehand laboratory was supported by program grants from the National Institute for Health Research to MK, SM and WHO and the British Heart Foundation under numbers RP-PG-0310-1002 and RG/09/12/28096; the laboratory also received funding from the NHS Blood and Transplant. MK was supported by Marie Curie funding from the NETSIM FP7 program, funded by the European Commission. OS received funding from the German Research Foundation (SO876/6-1, SFB914 B08, SFB1123 A06 \& B05) and NWO (91712303). SJ was supported by the National Human Genome Research Institute at the National Institutes of Health (U41 HG003751).

\section{Competing interests}

The authors declare that they have no competing interests.

\section{Authors' contribution}

CCB, SM and SdW performed experiments, analyzed and interpreted data and wrote the paper; MF and JMC analyzed and interpreted data and revised the manuscript; DA, JWA, RRS, OS, CW and JKW provided essential tools and revised the paper; MK and NJM analyzed data; MHP interpreted data and revised the manuscript; WHO and JWH designed research, analyzed and interpreted data and wrote the paper. 


\section{References}

1. World_Health_Organization. Factsheet Cardiovascular diseases Factsheet No: 317. 2013:http://www. who.int/mediacentre/factsheets/fs317/en/.

2. Mackman N. Triggers, targets and treatments for thrombosis. Nature. 2008;451:914-918.

3. Jackson SP. Arterial thrombosis: insidious, unpredictable and deadly. Nat Med. 2011;17:1423-1436.

4. Swieringa F, Kuijpers MJ, Heemskerk JW, van der Meijden PE. Targeting platelet receptor function in thrombus formation: the risk of bleeding. Blood Rev. 2014;28:9-21.

5. Westrick RJ, Ginsburg D. Modifier genes for disorders of thrombosis and hemostasis. J Thromb Haemost. 2009;7 Suppl 1:132-135.

6. Nieswandt B, Pleines I, Bender M. Platelet adhesion and activation mechanisms in arterial thrombosis and ischaemic stroke. J Thromb Haemost. 2011;9 (Suppl. 1):92-104.

7. Wei AH, Schoenwaelder SM, Andrews RK, Jackson SP. New insights into the haemostatic function of platelets. Br J Haematol. 2009;147:415-430.

8. Borissoff JI, Spronk HM, ten Cate H. The hemostatic system as a modulator of atherosclerosis. $\mathrm{N}$ Engl J Med. 2011;364:1746-1760.

9. Nieswandt B, Varga-Szabo D, Elvers M. Integrins in platelet activation. J Thromb Haemost. 2009;7 Suppl 1:206-209.

10. Denis CV, Dubois C, Brass LF, Heemskerk JW, Lenting PJ. Towards standardization of in vivo thrombosis studies in mice. J Thromb Haemost. 2011;9:1641-1644.

11. Zwaginga JJ, Sakariassen KS, Nash G, et al. Flow based assays for global assessment of haemostasis. Part 2: current methods and considerations for the future. J Thromb Haemost. 2006;4:2716-2717.

12. Emerson M. Refinement, reduction and replacement approaches to in vivo cardiovascular research. $\mathrm{Br} \mathrm{J}$ Pharmacol. 2010;161(4):749-754.

13. De Witt SM, Swieringa F, Cavill R, et al. Identification of platelet function defects by multi-parameter assessment of thrombus formation. Nat Commun. 2014;5:4257.

14. Hosokawa K, Ohnishi T, Sameshima H, et al. Analysing responses to aspirin and clopidogrel by measuring platelet thrombus formation under arterial flow conditions. Thromb Haemost. 2013;109:102-111.

15. Flamm MH, Colace TV, Chatterjee MS, et al. Multiscale prediction of patient-specific platelet function under flow. Blood. 2012;120:190-198.

16. Neeves KB, Onasoga AA, Hansen RR, et al. Sources of variability in platelet accumulation on type 1 fibrillar collagen in microfluidic flow assays. Plos One. 2013;7:e54680.

17. Li R, Diamond SL. Detection of platelet sensitivity to inhibitors of COX-1, P2Y ${ }_{1}$, and $P 2 Y_{12}$ using a whole blood microfluidic flow assay. Thromb Res. 2014;133:203-210.

18. Stegner D, Nieswandt B. Platelet receptor signaling in thrombus formation. J Mol Med. 2011;89:109-121. 19. Versteeg HH, Heemskerk JW, Levi M, Reitsma PS. New fundamentals in hemostasis. Physiol Rev. 2013;93:327-358.

20. McKenzie JE, Salanti G, Lewis SC, Altman DG. Meta-analysis and the Cochrane collaboration: 20 years of the Cochrane statistical methods group. Systematic Rev. 2013;2:80.

21. Cochrane Community. RevMan 5 2017. Available from: http://community.cochrane.org/tools/reviewproduction-tools/revman-5.

22. Croft D, Mundo AF, Haw R, et al. The Reactome pathway knowledgebase. Nucleid Acids Res. 2014;42:D472477.

23. Gieger C, Radhakrisknan A, Cvejic A, et al. New gene functions in megakaryopoiesis and platelet formation. Nature. 2011;480:201-208.

24. Chen L, Kostadima M, Martens JH, et al. Transcriptional diversity during lineage commitment of human blood progenitors. Science. 2014;345(6204):1251033.

25. Tijssen MR, Cvejic A, Joshi A, et al. Genome-wide analysis of simultaneous GATA1/2, RUNX1, FLI1, and SCL binding in megakaryocytes identifies hematopoietic regulators. Dev Cell. 2011;20(5):597-609.

26. Skarnes WC, Rosen B, West AP, et al. A conditional knockout resource for the genome-wide study of mouse gene function. Nature. 2011;474(7351):337-342.

27. Koenen RR, von Hundelshausen P, Nesmelova IV, et al. Disrupting functional interactions between platelet chemokines inhibits atherosclerosis in hyperlipidemic mice. Nat Med. 2009;15:97-103.

28. Weber C, Soehnlein O. ApoE controls the interface linking lipids and inflammation in atherosclerosis. J 
Clin Invest. 2011;121:3825-3827.

29. Drechsler M, de Jong R, Rossaint J, et al. Annexin A1 counteracts chemokine-induced arterial myeloid cell recruitment. Circ Res 2015;116:827-835.

30. Kuijpers MJ, Mattheij NJ, Cipolla L, etal. Platelet CD40L modulates thrombusgrowth via phosphatidylinositol 3-kinase $\beta$, and not via CD40 and IKB kinase $\alpha$. Arterioscler Thromb Vasc Biol. 2015;35(6):1374-1381.

31. De Witt SM, Swieringa F, Cosemans JM, Heemskerk JW. Thrombus formation on microspotted arrays of thrombogenic surfaces. Nat Protocol Exchange. 2014;3309.

32. Schroll JB, Moustgaard R, Gotzsche PC. Dealing with substantial heterogeneity in Cochrane reviews. Cross-sectional study. BMC Med Res Methodol. 2011;11:22.

33. Jupe S, Akkerman JW, Soranzo N, Ouwehand WH. Reactome: a curated knowledgebase of biological pathways: megakaryocytes and platelets. J Thromb Haemost 2012;10:2399-2402.

34. Rivadeneyra L, Pozner RG, Meiss R, et al. Poly $(\mathrm{I}: \mathrm{C})$ downregulates platelet production and function through type I interferon. Thromb Haemost. 2015;114(5):982-993.

35. Schmidt EM, Schmid E, Münzer P, et al. Chorein sensitivity of cytoskeletal organization and degranulation of platelets. FASEB J. 2013;27(7):2799-2806.

36. GeneCards. The human gene database. wwwgenecardsorg. 2015.

37. Eckly $A$, Hechler $B$, Freund $\mathrm{M}$, et al. Mechanisms underlying $\mathrm{FeCl}_{3}$-induced arterial thrombosis. J Thromb Haemost. 2011;9(4):779-789.

38. Mangin P, Yap CL, Nonne C, et al. Thrombin overcomes the thrombosis defect associated with platelet GPVI/FcRy deficiency. Blood. 2006;107(11):4346-4353.

39. Dubois C, Panicot-Dubois L, Gainor JF, Furie BC, Furie B. Thrombin-initiated platelet activation in vivo is VWF independent during thrombus formation in a laser injury model. J Clin Invest. 2007;117(4):953-960.

40. Cosemans JM, Kuijpers MJ, Lecut C, et al. Contribution of platelet glycoprotein VI to the thrombogenic effect of collagens in fibrous atherosclerotic lesions. Atherosclerosis. 2005;181:19-27.

41. Reininger AJ, Bernlochner I, Penz SM, et al. A 2-step mechanism of arterial thrombus formation induced by human atherosclerotic plaques. J Am Coll Cardiol. 2010;55:1147-1158. 


\section{Data Files}

Data File 1. Primary input of included mouse studies, variables, published measurements, (sub)scores and references.

Data File 2. Full input data of meta-analysis using RevMan 5.1 for 6 mouse genes. Data File 3. Mean output scores per mouse gene of modification on thrombus formation (classes I-III) and tail bleeding (class IV) with details on genetics and suggested functions.

Data File 4. Mouse-human comparison of orthologous genes with Uniprot assignments for pathway and network analysis. In addition, Reactome pathway-topology analysis indicating coverage of best matches per pathway.

Data File 5. Characteristics of human protein interaction network in thrombosis and hemostasis (PITH), including seeded and novel proteins and numbers of edges. In addition, transcription profiles of genes along human megakaryocyte lineage.

Datafiles are available upon request. 


\section{Supplements}

A

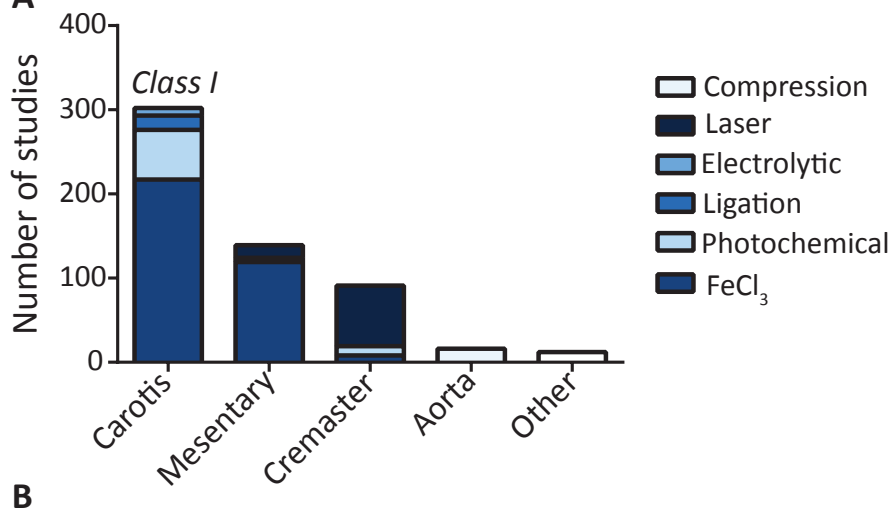

B
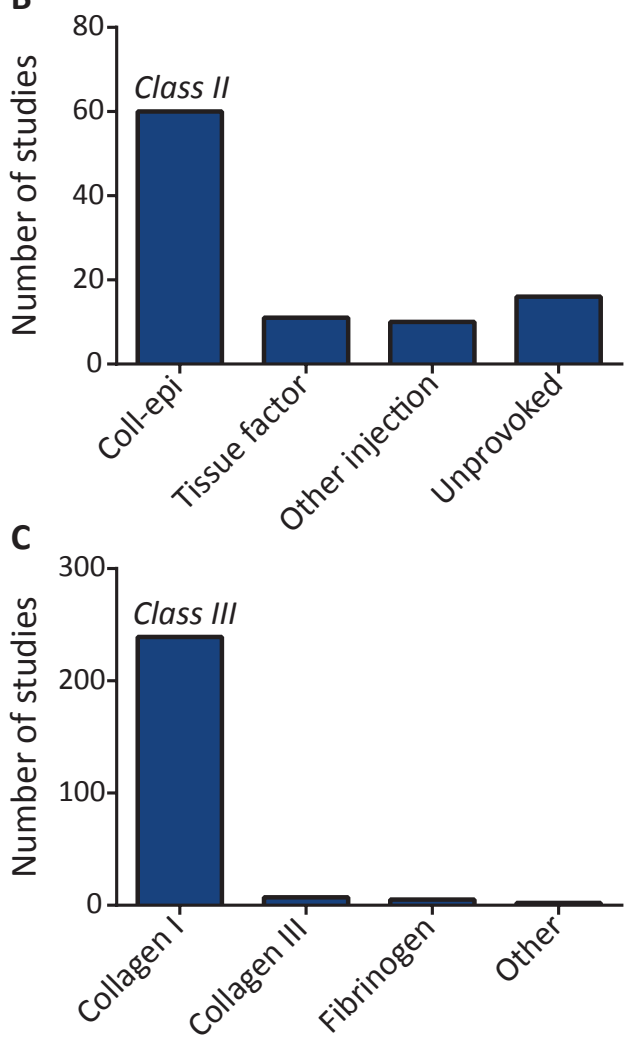

Suppl. Figure 1. Numbers of studies analyzed in classes I-III with main experimental variables. A, Class I studies grouped with separation according to vascular bed or type of injury. B, Class II studies grouped with separation according to type of systemic intervention. C, Class III studies with separation for type of thrombogenic surface. 


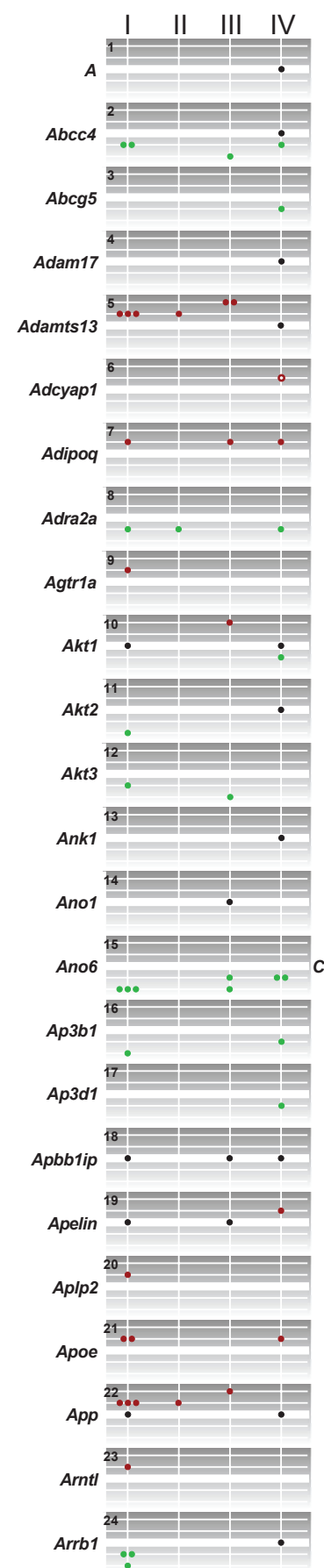

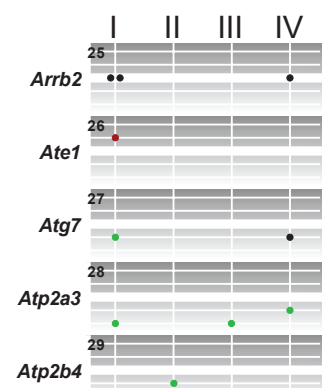
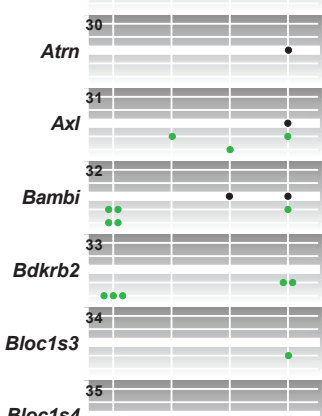

Bloc1s4
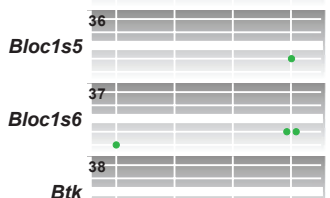

Btk
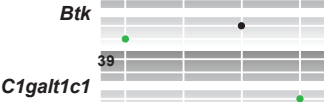

$C 22^{40}$
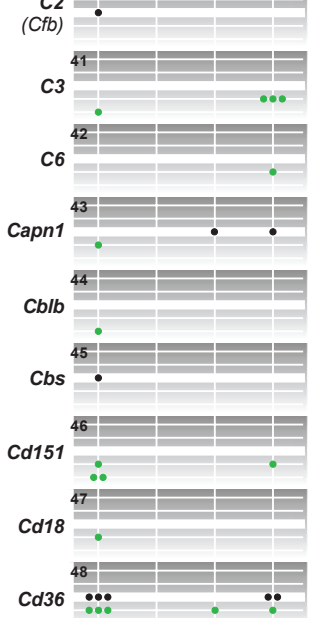
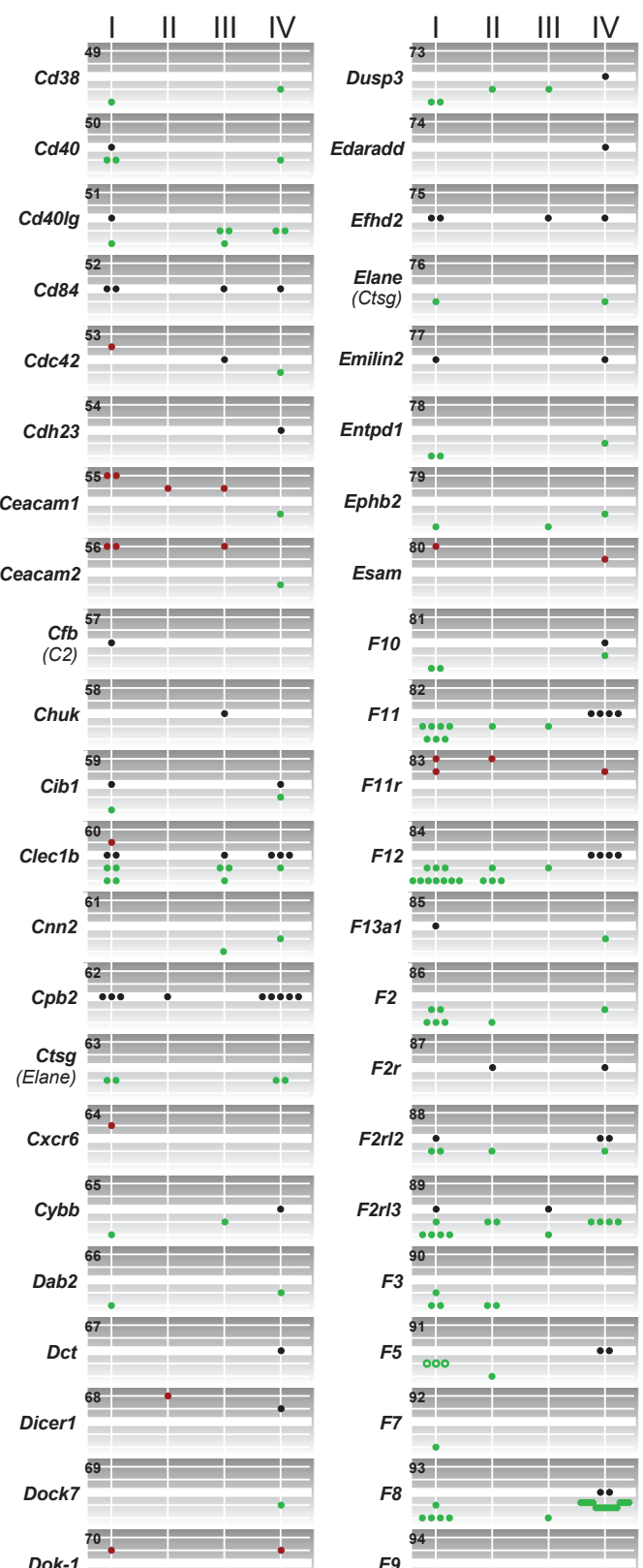

Dok-1

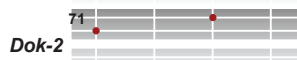

\begin{tabular}{l|l|l|}
\hline${ }^{72}$ & \\
Dtnbp 1 & \\
\hline
\end{tabular}
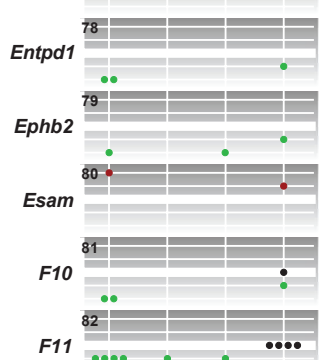
$\because \because$
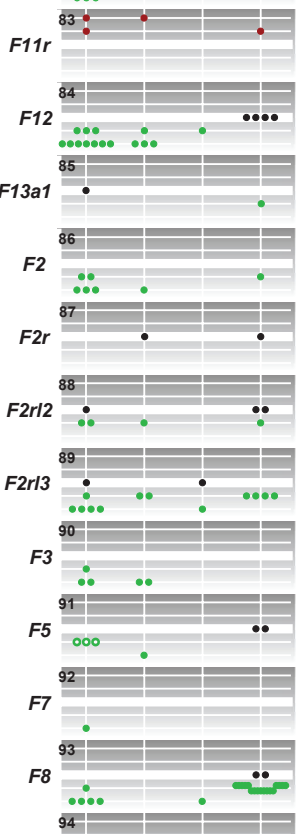

F9

9

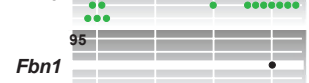

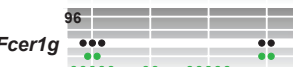



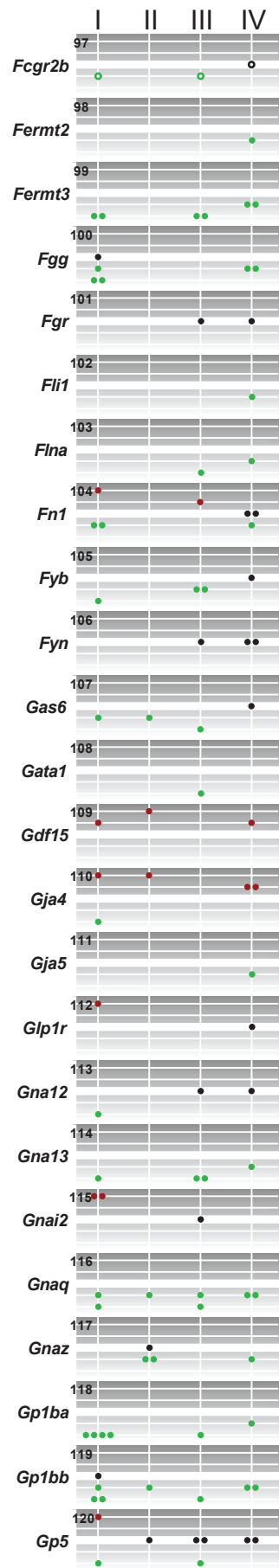
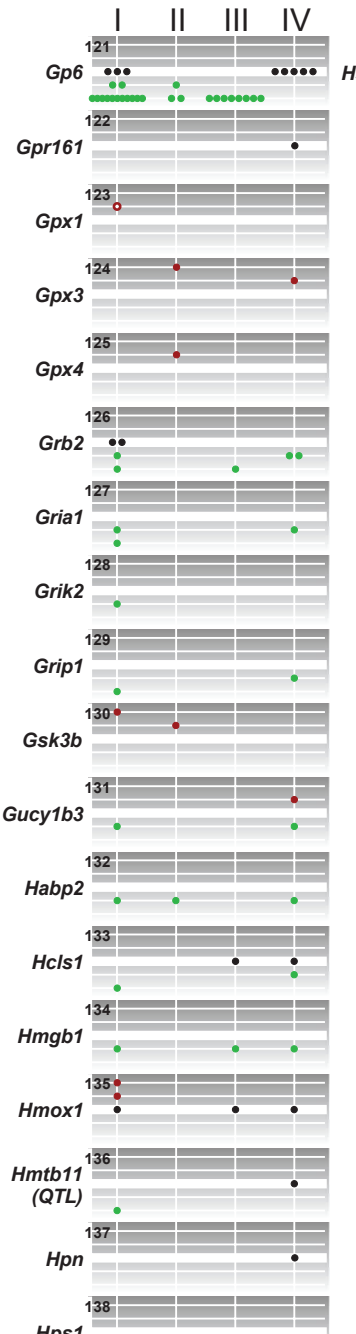

Hps1
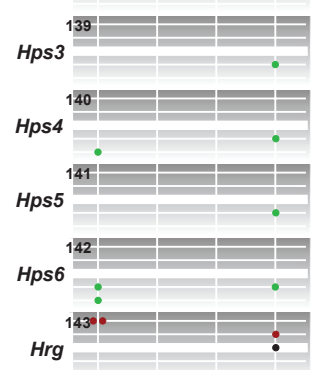

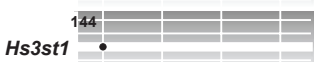
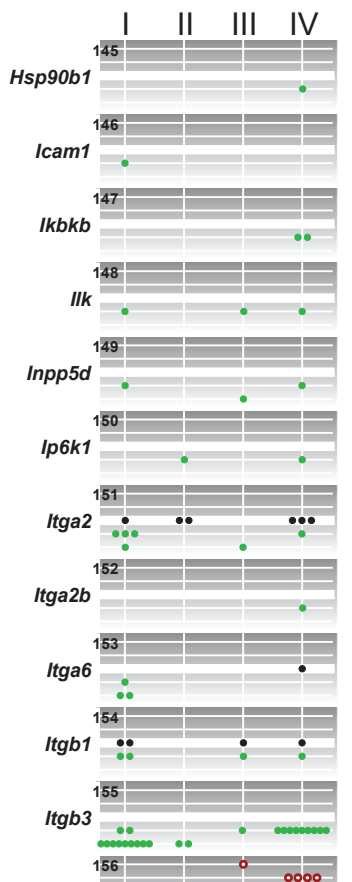

Jak2 $00 \quad: \quad 0000$
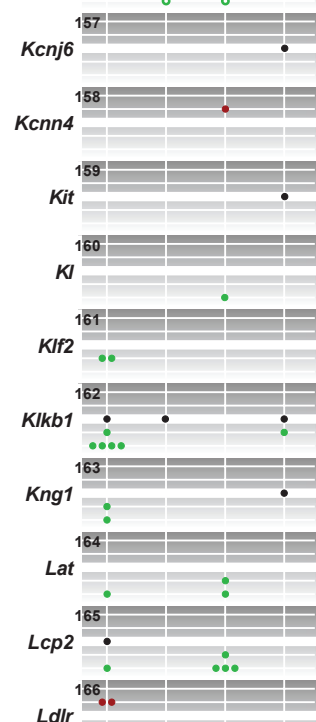

Ldlr
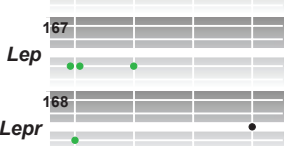

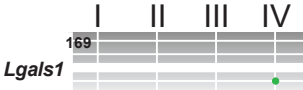
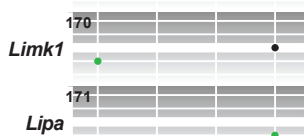

-
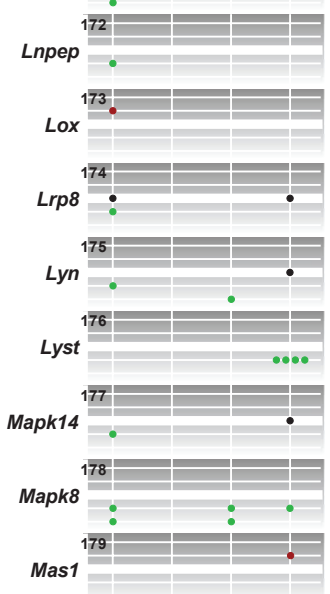

Masp1 \begin{tabular}{r|r|r|}
180 & \\
\hline
\end{tabular}

Mbl1 ${ }^{181^{\circ}}$ (Mb/2)

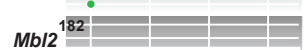

(Mbl1)
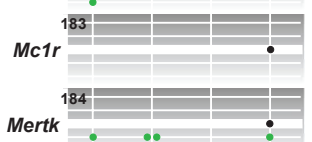

\begin{tabular}{r|r|r|}
185 & \\
\hline
\end{tabular}

Mfap2 …
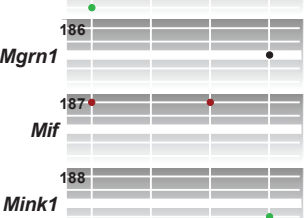

Mink1

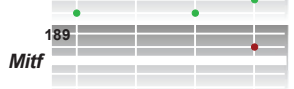

\begin{tabular}{l|l|l} 
Mkl1 $^{190}$ & \\
&
\end{tabular}

(Mkl2) = :

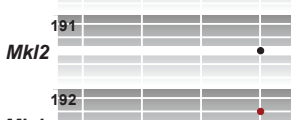

MIph 


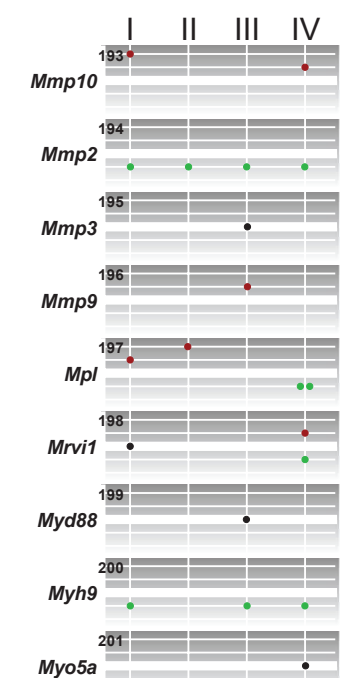

Nbeal2 $202+\frac{}{202}+$

$\mathrm{Nfe}^{203} \cdot \mathbf{0}$
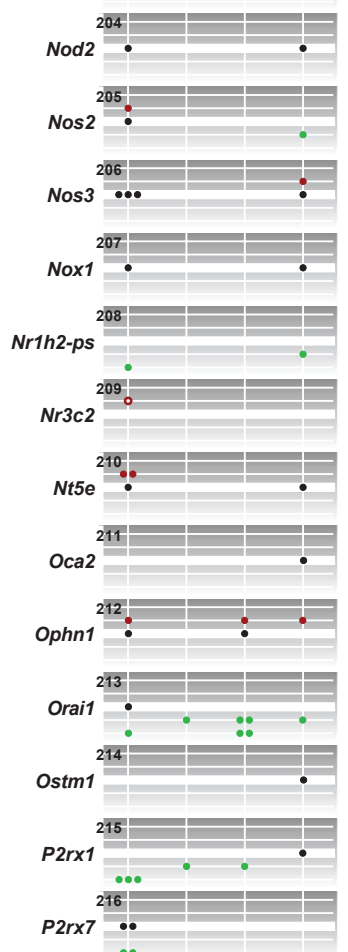
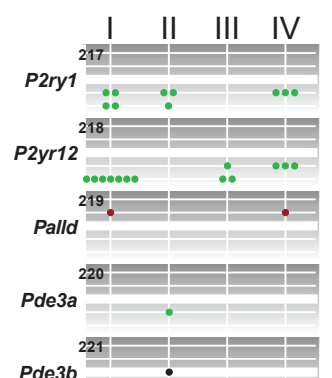

Pde3b
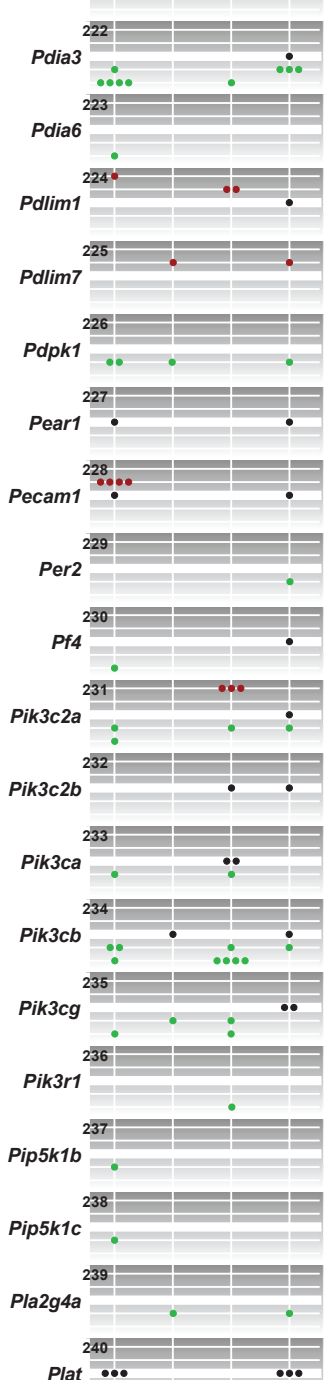
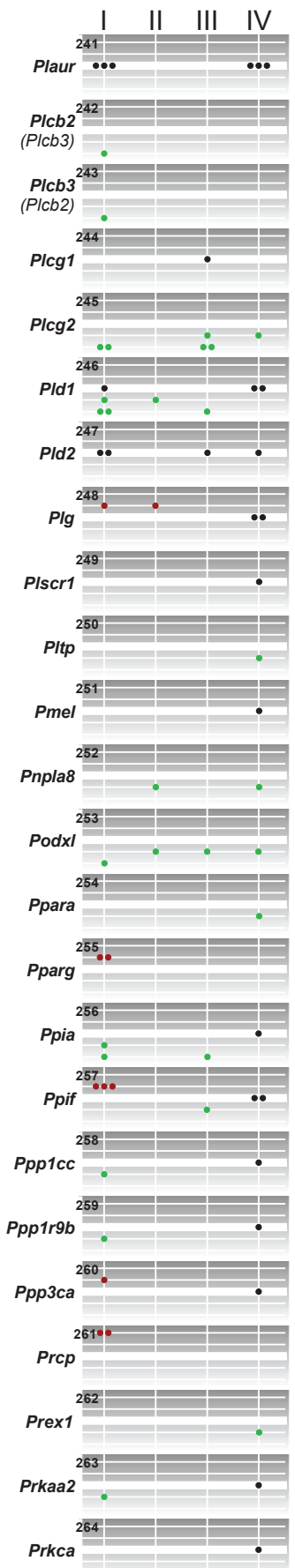
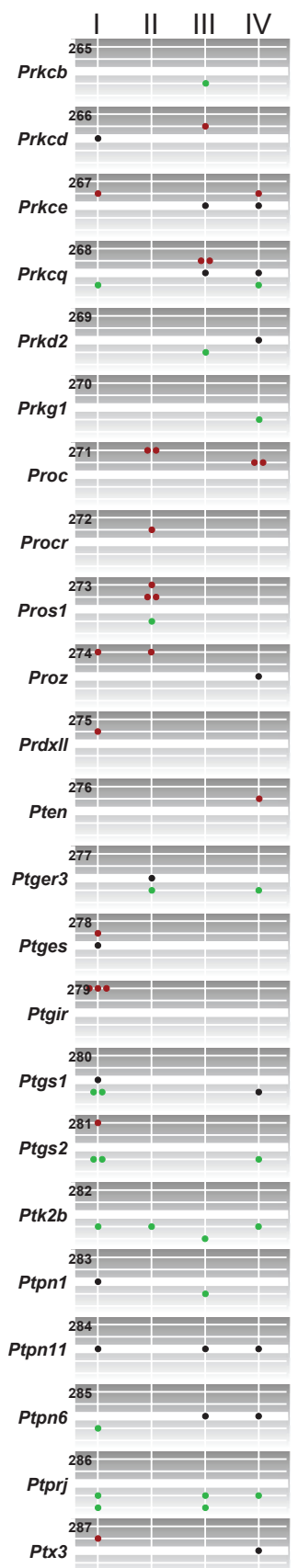

$R^{288}{ }^{288} a^{-}$ 

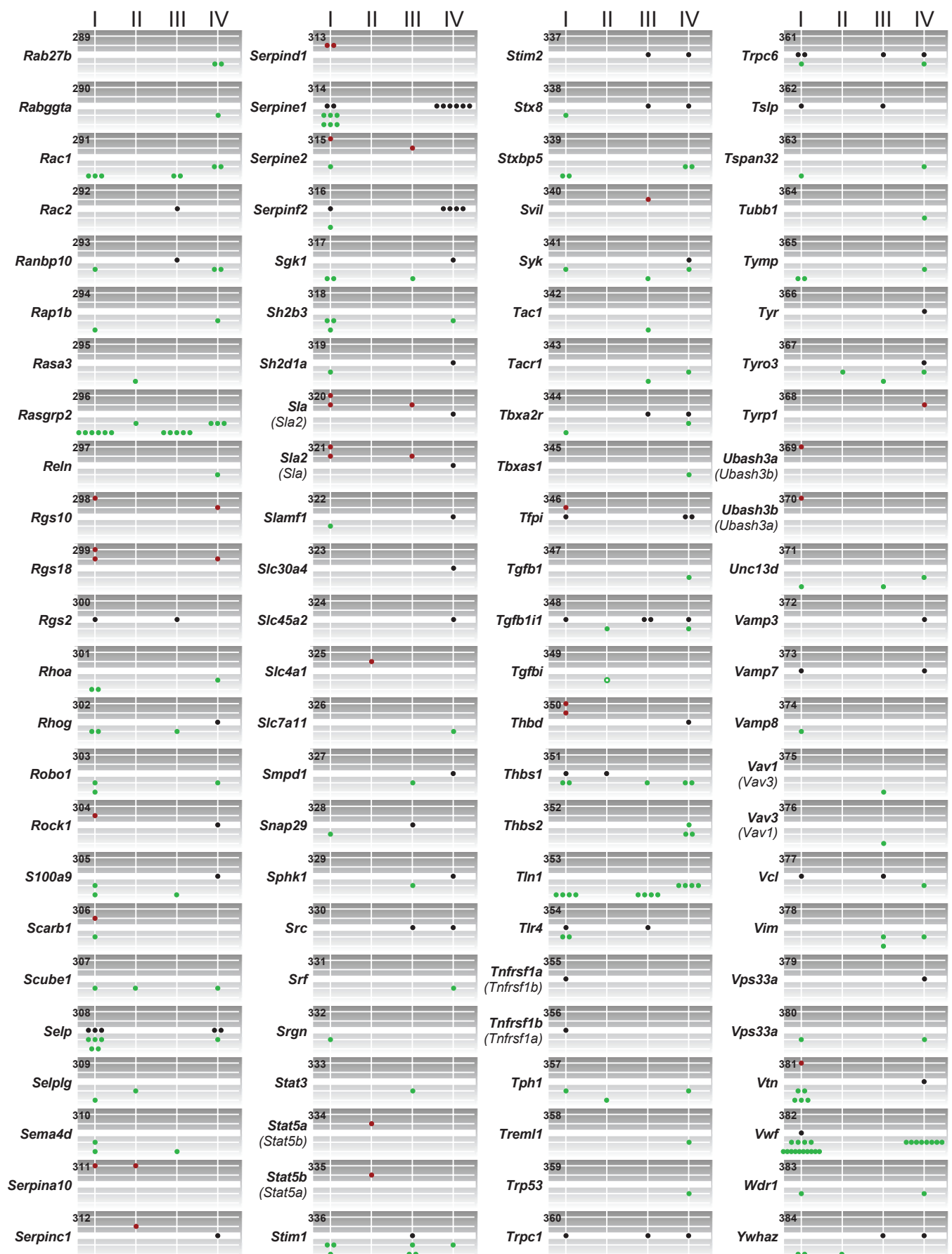

(Vav3) =-
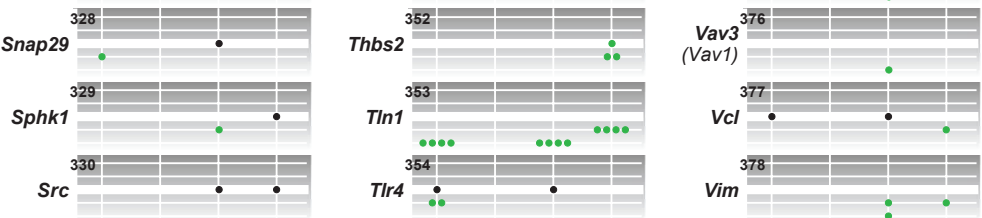

(Vav1)
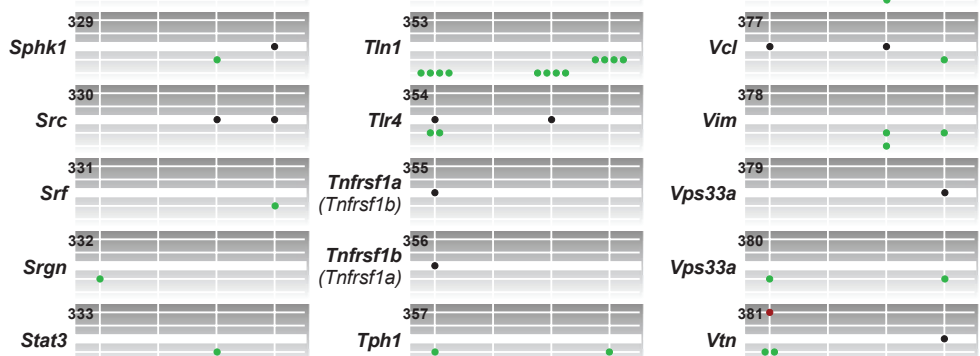

Stat5a $a^{334}$ (Stat5b) = 19:

Stat5b $^{335}$ ! (Stat5a)
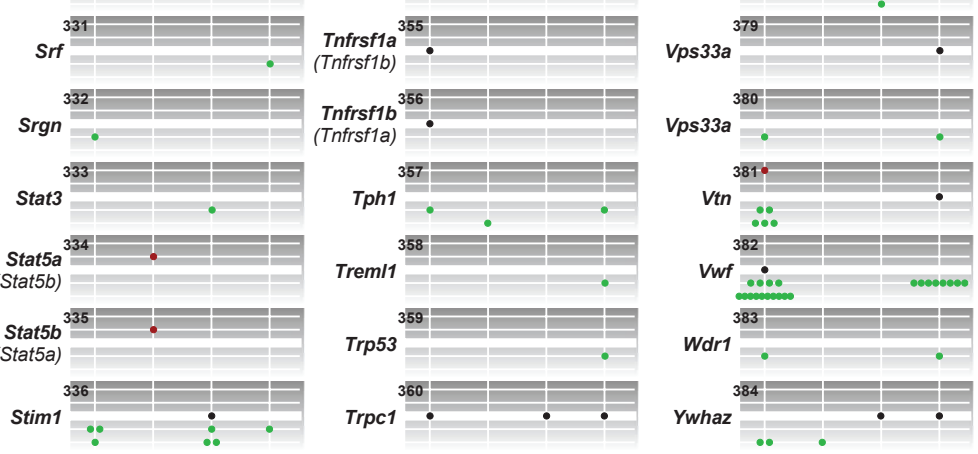


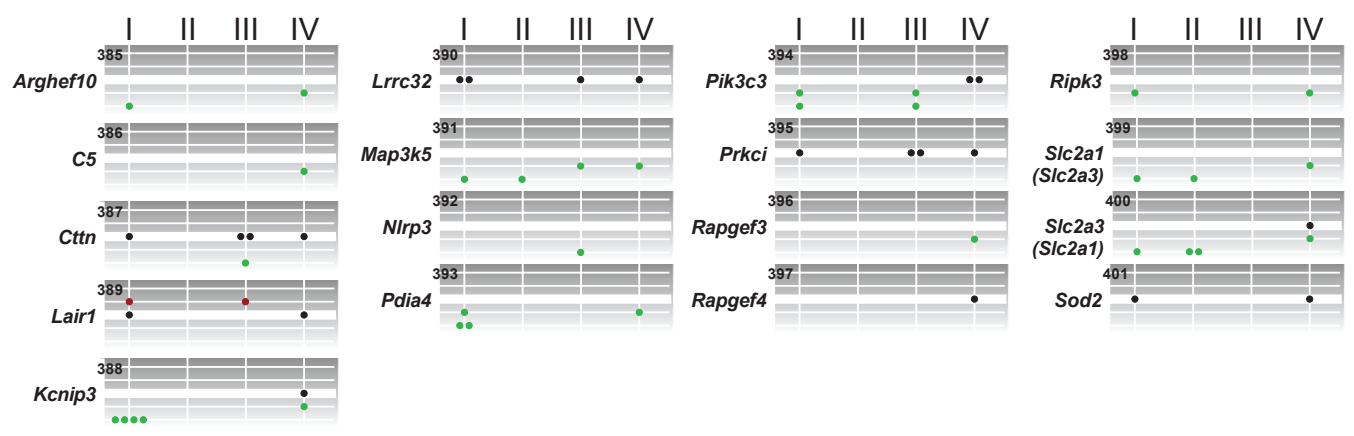

Suppl. Figure 2. Overview per mouse gene and study of scores in classes I-IV. Individual studies are described and scored in Data File 1. 


\section{Probabilities: 1e+00 3e-01 1e-013e-02 1e-02 3e-03 1e-03 3e-04 1e-04 3e-05 1e-05 3e-06 1e-06 3e-07 1e-07 3e-08} $1 \mathrm{e}-083 \mathrm{e}-09$ 1e-09 $3 \mathrm{e}-10$ 2

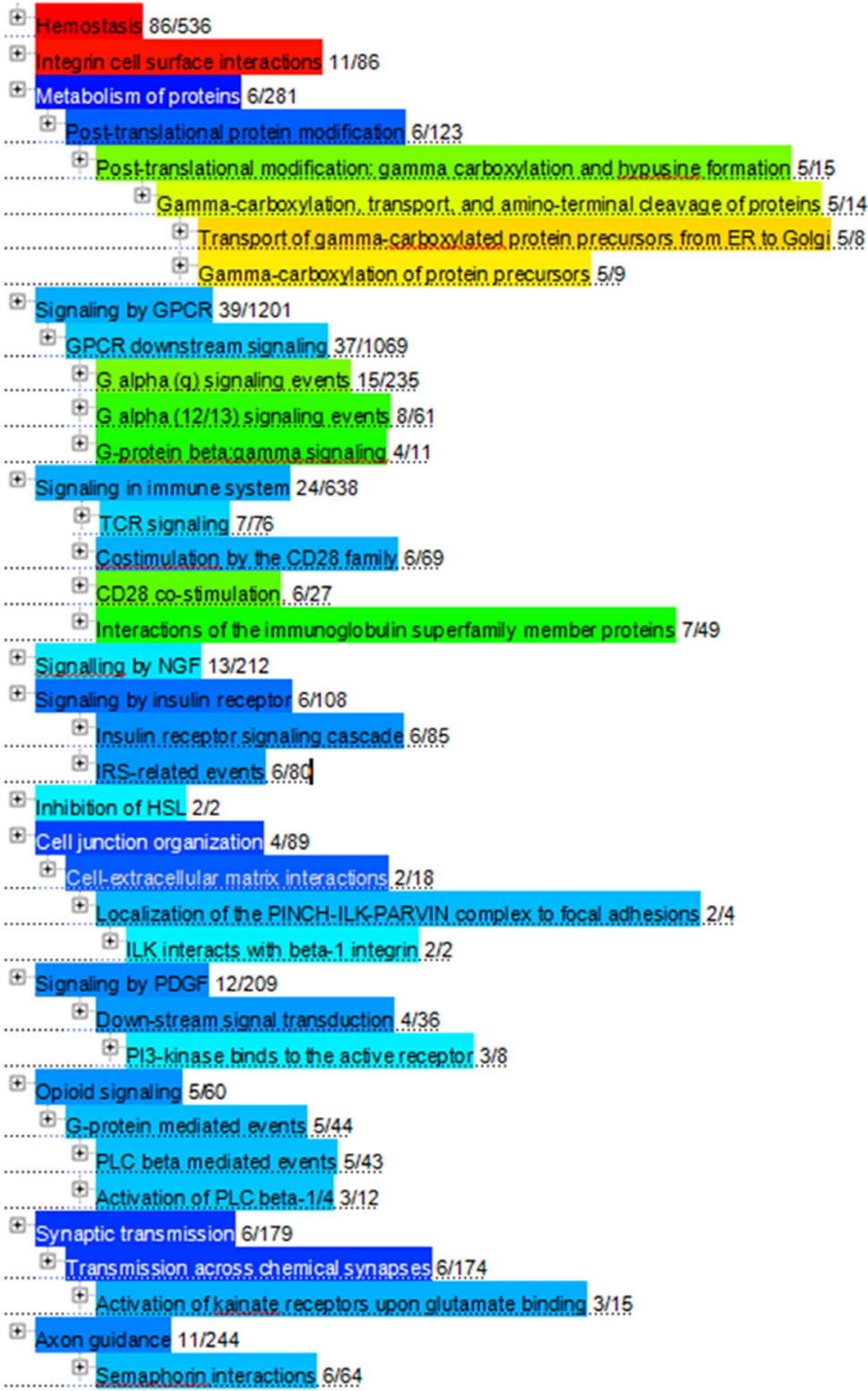

Suppl. Figure 3. Reactome hierarchical over-representation analysis of orthologous human proteins corresponding to mouse genes with non-zero scores for arterial thrombus formation and/or bleeding. Numbers per pathway (Reactome event) are given. Each event is colored according to the probability of seeing the given number or more proteins in the pathway by chance. The top-level (root) pathways are ordered according to the lowest p-value of their components. Total number of events assessed was 5187, number of matching events was 375, number of proteins (genes) matching submitted identifiers was 105. 


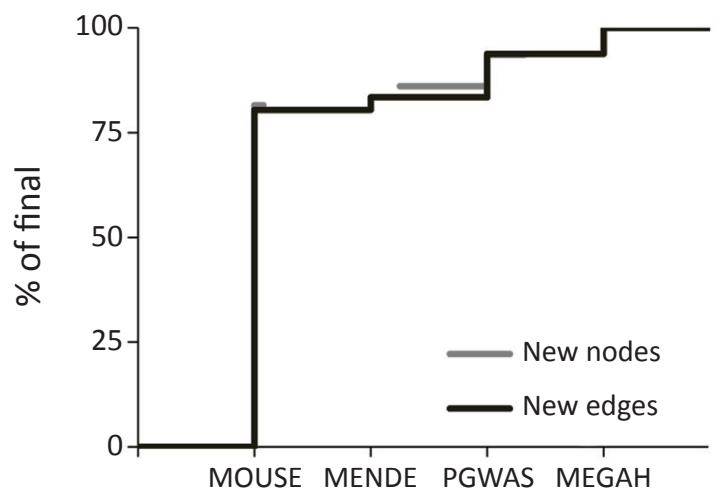

Suppl. Figure 4. Buildup of protein-protein interaction network. Networks were stepwise constructed in Cytoscape, using the following sets of seed nodes: human orthologues of modifying mouse proteins (MOUSE, 282 genes \& proteins). mendelian disorders (MENDE, 72 proteins), Platelet GWAS (PGWAS, 68 proteins), Megakaryocytopoiesis hubs (MEGAH, 65 proteins). Note that 62 of these proteins were present in more than one list (see Data File 5). In each network built, final number of total nodes was 3,119 (2,649 connected to modifying mouse proteins), and final number of total edges was 18,452 . Shown are percentages of new nodes and new edges per added seed nodes. 
Suppl. Table 1. Classification and grouping of analyzed mouse studies. Indicated are the assignment of studies to classes and groups, as well as the evaluated output parameters of thrombus formation, thromboembolism or tail bleeding of indicated classes.

\begin{tabular}{|c|c|}
\hline Class I: in vivo arterial thrombo & \\
\hline \multicolumn{2}{|l|}{ Groups compared (1-12) } \\
\hline Carotid artery injury & $\mathrm{FeCl}_{3}(1)$; photochemical (2); ligation (3); electrolytic (4) \\
\hline Aorta injury & compression (5) \\
\hline Mesenteric artery injury & $\mathrm{FeCl}_{3}(6)$; photochemical (7); laser (8) \\
\hline Cremaster artery injury & $\mathrm{FeCl}_{3}$ (9); photochemical (10); laser (11) \\
\hline Other artery/injury & any (12) \\
\hline \multicolumn{2}{|l|}{ Parameters compared (a-c) } \\
\hline Time-dependent parameters & time to platelet adhesion, time to occlusion or thrombus (a) \\
\hline Mass-dependent parameters & $\begin{array}{l}\text { extent of platelet adhesion (bi); extent of occlusion (bii); } \\
\text { number, size or volume of thrombi (biii) }\end{array}$ \\
\hline Stability parameters & number or frequency of emboli \\
\hline \multicolumn{2}{|l|}{ Class II: in vivo thromboembolism } \\
\hline \multicolumn{2}{|l|}{ Groups compared (20-23) } \\
\hline Provoked $(d)$ & $\begin{array}{l}\text { injection of collagen/epinephrine (20); tissue factor (21); or } \\
\text { other agent (22) }\end{array}$ \\
\hline Unprovoked (e) & pre/after birth (23) \\
\hline \multicolumn{2}{|l|}{ Parameters compared } \\
\hline Thromboembolism parameters & $\begin{array}{l}\text { percentage death, time to death, residual platelet count (d); } \\
\text { unprovoked thrombosis or bleeding (e) }\end{array}$ \\
\hline \multicolumn{2}{|c|}{ Class III: in vitro thrombus formation } \\
\hline \multicolumn{2}{|l|}{ Groups compared (30-34) } \\
\hline Adhesive surface & $\begin{array}{l}\text { collagen I (30); collagen III (31); VWF (32); fibrinogen (33); } \\
\text { other (34) }\end{array}$ \\
\hline \multicolumn{2}{|l|}{ Parameters compared $(\boldsymbol{f}$-h) } \\
\hline Time-dependent parameters & time to platelet adhesion, time to thrombus formation (f) \\
\hline Mass-dependent parameters & $\begin{array}{l}\text { extent of platelet adhesion (gi); surface area coverage (gii); } \\
\text { number, size or volume of thrombi (giii) }\end{array}$ \\
\hline Stability parameters & number or frequency of emboli (h) \\
\hline Class IV: bleeding time & tail bleeding time \\
\hline
\end{tabular}


Suppl. Table 2. Correlation analysis of intra-study parameters scored on 3-point or 5-point scale. Indicated are numbers of studies per parameter, Kendall's Tau correlation coefficients, and $P$ values. A, Comparison of mass-dependent sub-parameters in class I studies (bi, platelet adhesion; bii, extent of occlusion; biii, thrombus size, volume or number). B, Comparison of mass-dependent sub-parameters in class III studies (gi, platelet adhesion; gii, surface-area-coverage; giii, thrombus size, volume or number). C, Comparison of time-dependent $(\boldsymbol{a})$, mass-dependent $(\boldsymbol{b})$, and stability $(\boldsymbol{c})$ parameters in class I studies. Time-dependent parameters $(a / f)$ included time to occlusion and time to thrombus formation; stability parameters $(c / h)$ included number of emboli. D, Comparison of time-dependent $(\boldsymbol{e})$, mass-dependent $(\boldsymbol{f})$, and stability $(\boldsymbol{g})$ parameters for all class III studies.

A

\begin{tabular}{l|rrrr}
\multicolumn{2}{c}{$\begin{array}{l}\text { Number of studies } \\
\end{array}$} & bi & bii & \multicolumn{2}{c}{ biii } \\
\hline bi & 60 & & \\
bii & 9 & 86 & \\
biii & 19 & 10 & 151
\end{tabular}

Kendall's $\tau$ (3-point scale)

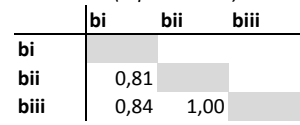

P-value (3-point scale)

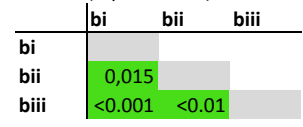

Kendall's $\tau$ (5-point scale)

\begin{tabular}{l|rrr} 
& bi & bii & biii \\
\hline bi & & \\
bii & 0,50 & \\
biii & 0,78 & 0,79
\end{tabular}

$P$-value (5-point scale)

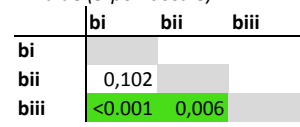

B

\begin{tabular}{l|rrrr}
\multicolumn{6}{l}{$\begin{array}{l}\text { Number of studies } \\
\end{array}$} & gi & gii & \multicolumn{2}{c}{ giii } \\
\hline gi & 36 & & \\
gii & 20 & 171 & \\
giii & 12 & 69 & 103
\end{tabular}

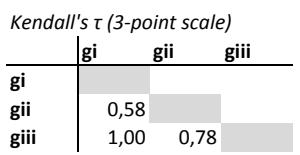

P-value (3-point scale)
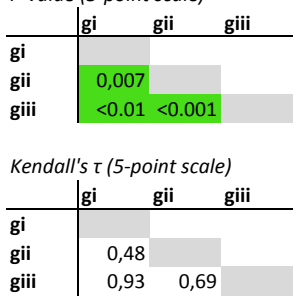

P-value (5-point scale)

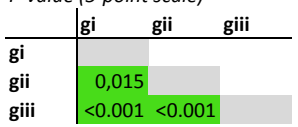

C

\begin{tabular}{l|r|rrr}
\multicolumn{6}{c}{ Number of studies } \\
\multicolumn{1}{c|}{} & a & b & c & \\
\hline a & 377 & & \\
b & 93 & 261 & \\
c & 46 & 46 & 78
\end{tabular}

Kendall's $\tau$ (3-point scale)

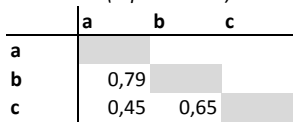

P-value (3-point scale)

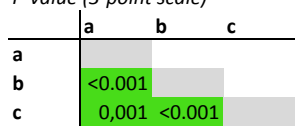

Kendall's $\tau$ (5-point scale)

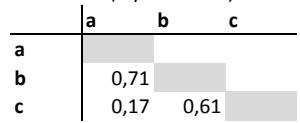

P-value (5-point scale)

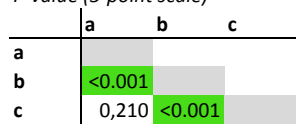

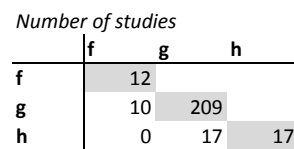

\begin{tabular}{l|rrr}
\multicolumn{4}{c}{ Kendall's $\tau$ (3-point scale) } \\
& $\mathbf{f}$ & $\mathbf{g}$ & $\mathbf{h}$ \\
\hline $\mathbf{f}$ & & & \\
$\mathbf{g}$ & 0,67 & & \\
$\mathbf{h}$ & - & 0,81
\end{tabular}

$P$-value (3-point scale)

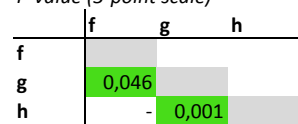

\begin{tabular}{l|rrr}
\multicolumn{4}{c}{ Kendall's $\tau$ (5-point scale) } \\
& $\mathbf{f}$ & $\mathbf{g}$ & $\mathbf{h}$ \\
\hline $\mathbf{f}$ & & & \\
$\mathbf{g}$ & 0,56 & & \\
$\mathbf{h}$ & - & 0,75
\end{tabular}

\begin{tabular}{l|rrr}
\multicolumn{4}{l}{ P-value (5-point scale) } \\
& $\mathbf{f}$ & g & h \\
\hline $\mathbf{f}$ & & & \\
$\mathbf{g}$ & 0,072 & & \\
$\mathbf{h}$ & - & 0,001
\end{tabular} 
Suppl. Table 3. Correlation analysis of mean study scores per class on 3-point or 5-point scale. A, Comparison of studies published in the same paper (identical mouse strain). B, Comparison of all published studies per gene (loss-of function mutations only). Panels show numbers of studies or genes compared, Kendall's Tau correlation coefficients, and $P$ values (3-point or 5-point scores, as indicated).

A

\begin{tabular}{|c|c|c|c|c|}
\hline \multicolumn{5}{|c|}{ Number of studies } \\
\hline & | & I & & \\
\hline 1 & & 539 & & \\
\hline II & & 48 & 105 & \\
\hline III & & 132 & 14 & 253 \\
\hline
\end{tabular}

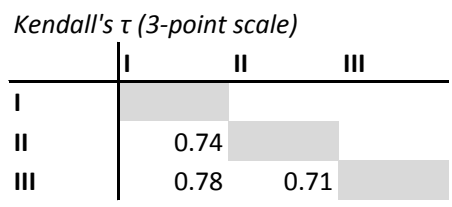

P-value (3-point scale)

\begin{tabular}{l|lll} 
& I & II & III \\
\hline I & & & \\
II & $<0.001$ & & \\
III & $<0.001$ & 0.006
\end{tabular}

Kendall's $\tau$ (5-point scale)

\begin{tabular}{l|lll} 
& I & II & III \\
\hline I & & & \\
II & 0.65 & & \\
III & 0.69 & 0.33
\end{tabular}

P-value (5-point scale)

\begin{tabular}{l|lll} 
& I & II & III \\
\hline I & & & \\
II & $<0.001$ & & \\
III & $<0.001$ & 0.165
\end{tabular}

B

Number of genes

\begin{tabular}{l|rrrr} 
& I & II & \multicolumn{2}{|c}{ III } \\
\hline I & 261 & & \\
II & 49 & 75 & \\
III & 114 & 29 & 144
\end{tabular}

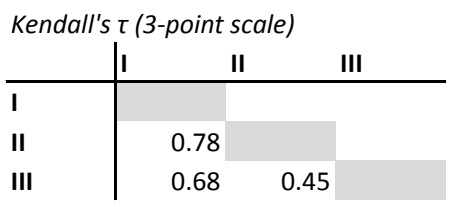

P-value (3-point scale)

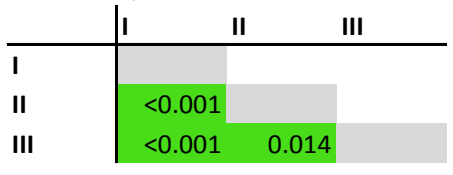

Kendall's $\tau$ (5-point scale)

\begin{tabular}{|c|c|c|c|c|}
\hline & I & & II & III \\
\hline 1 & & & & \\
\hline II & & 0.51 & & \\
\hline III & & 0.60 & 0.10 & \\
\hline
\end{tabular}

$P$-value (5-point scale)

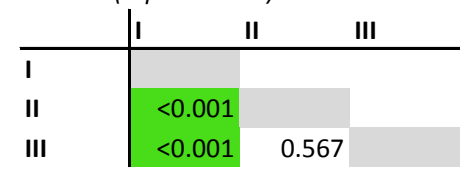


Suppl. Table 4. Numbers of scored mouse genes per class and group. For full description of classes and groups, see Suppl. Table 1.

\begin{tabular}{|c|c|c|c|c|c|c|c|c|c|c|c|c|c|}
\hline & & 1 & 3 & 4 & 5 & 6 & 7 & 8 & 9 & 10 & 11 & & 12 \\
\hline 1 & Carotis $\mathrm{FeCl}_{3}$ & 149 & & & & & & & & & & & \\
\hline 2 & Carotis photochemical & 19 & 38 & & & & & & & & & & \\
\hline 3 & Carotis ligation & 7 & 3 & 13 & & & & & & & & & \\
\hline 4 & Carotis electrolytical & 4 & 0 & 3 & 7 & & & & & & & & \\
\hline 5 & Aorta compression & 5 & 1 & 0 & 0 & 15 & & & & & & & \\
\hline 6 & Mesentery $\mathrm{FeCl}_{3}$ & 38 & 9 & 7 & 2 & 11 & 89 & & & & & & \\
\hline 7 & Mesentery photochemical & 1 & 1 & 0 & 0 & 0 & 1 & 3 & & & & & \\
\hline 8 & Mesentery laser & 7 & 0 & 1 & 1 & 1 & 5 & 0 & 13 & & & & \\
\hline 9 & Cremaster $\mathrm{FeCl}_{3}$ & 2 & 0 & 1 & 1 & 0 & 4 & 0 & 2 & 4 & & & \\
\hline 10 & Cremaster photochemical & 5 & 0 & 0 & 0 & 0 & 3 & 0 & 0 & 0 & 11 & & \\
\hline 11 & Cremaster laser & 17 & 4 & 4 & 3 & 2 & 18 & 0 & 4 & 4 & 2 & 55 & \\
\hline 12 & Other & 3 & 3 & 2 & 1 & 2 & 6 & 0 & 3 & 1 & 0 & 1 & 11 \\
\hline
\end{tabular}

Class II (number of genes)

\begin{tabular}{l|l|rrrrr} 
& $\mathbf{2 0}$ & $\mathbf{2 1}$ & $\mathbf{2 2}$ & $\mathbf{2 3}$ \\
\hline $\mathbf{2 0}$ & Injection collagen-epinephrine & 47 & & & \\
$\mathbf{2 1}$ & Injection tissue factor & 2 & 10 & & \\
$\mathbf{2 3}$ & Injection other & 1 & 0 & 10 & \\
& Systemic other & 0 & 1 & 0 & 12
\end{tabular}

Class III (number of genes)

\begin{tabular}{l|l|rrrrr} 
& $\mathbf{3 0}$ & $\mathbf{3 1}$ & $\mathbf{3 2}$ & $\mathbf{3 3}$ & $\mathbf{3 4}$ \\
\hline $\mathbf{3 0}$ & Collagen I & 142 & & & & \\
$\mathbf{3 1}$ & Collagen III & 4 & 7 & & & \\
$\mathbf{3 2}$ & von Willebrand factor & 3 & 0 & 3 & & \\
$\mathbf{3 3}$ & Fibrinogen & 2 & 1 & 0 & 3 & \\
$\mathbf{3 4}$ & Other & 1 & 0 & 0 & 0 & 2
\end{tabular}

Class IV (number of genes)

\begin{tabular}{l|l|l}
\multicolumn{2}{l|}{} & $\mathbf{4 0}$ \\
\hline $\mathbf{4 0}$ & Tail bleeding & 292
\end{tabular}





\section{Chapter 9}

General Discussion 
As stated in Chapter 1, the overall goal of the studies was to better understand the consequences of an acquired impairment or recovery in platelet function in various physiological and clinical settings. This required further investigation on the activation processes of platelets, either isolated or in whole blood, during the process of thrombus formation. In the next paragraphs, I will discuss how the findings of this thesis have led to new insights into the causes of structural and functional heterogeneity between platelets. Subsequently, I will confer mechanisms by which an acquired low platelet count or platelet dysfunction can lead to impaired hemostasis, and how these can be overcome. In the last section, I will describe new insights arising from the synthesis study describing the hemostasis and thrombosis balance in genetically modified mice.

\section{Heterogeneity in platelet structure and function}

It has been recognized for more than a decade that substantial differences exist among platelets. ${ }^{1-3}$ The review Chapter 2 describes in detail how and why platelets from a given subject are heterogeneous in terms of structural characteristics (e.g., platelet size and receptor densities) and functional features (e.g., forming populations of aggregating, procoagulant and coated platelets). Given the life span of human platelets of 7-10 days, ${ }^{4}$ both younger (juvenile or reticulated) and older platelets are circulating. It has been proposed that the juvenile platelets are larger in size and are more reactive towards agonists than older platelets. 5 Most of the evidence has come from studies in which juvenile platelets were (weakly) labeled with thiazole orange, an mRNA probe with a debated specificity. ${ }^{7}$ In Chapter 3, we introduced a new method to identify juvenile platelets, namely by staining with fluorescently labeled oligo-dT, which directly binds to the poly-A tail of mRNA species. Using this probe we were able to demonstrate a regain of function in the newly formed platelets from patients, who stopped taking the irreversible $\mathrm{P} 2 \mathrm{Y}_{12}$ receptor antagonist prasugrel. Hence, in this specific setting we found a higher reactivity of (unblocked) juvenile platelets than their older counterparts. Whether these juvenile platelets are larger was not apparent from the flow cytometric analysis.

Although juvenile platelets were more reactive, by themselves they were unable to compensate for the impairment in thrombus and fibrin formation due to a low platelet count despite a relatively high number of juvenile platelets (immune thrombocytopenia, Chapter 4). While we find that during antiplatelet therapy and the offset period, irreversible $\mathrm{P}_{2} \mathrm{Y}_{12}$ inhibition impairs the reactivity of the juvenile platelets, several other studies have shown a positive correlation between the percentage of juvenile platelets and on-treatment platelet reactivity. ${ }^{8-10}$ Thereby, it was suggested that, due to a high platelet turnover, juvenile platelets are incompletely inhibited by antiplatelet therapy and, thus, contribute to high on-treatment platelet reactivity. ${ }^{8,10}$ However, our data in Chapter 3 are not supportive of this hypothesis, in that the reactivity of juvenile platelets during the initial days of the offset period was still low and only gradually increased in 
time. This observation agrees with the evidence from other authors that the intrinsic structural properties of juvenile platelets, rather than a high turnover, explain a high ontreatment activity. ${ }^{11}$

According to their presumed functions, activated platelets can be separated into the - partly overlapping - populations of adhesive, aggregating, procoagulant and coated platelets. Each of these populations is characterized by a specific set of surface markers and can be distinguished in a thrombus (Figure 1)..$^{1,12,13} \mathrm{How}$ individual platelets transform into one of these populations is not fully clear. In Chapter 2 we reviewed the current understanding of platelet heterogeneity and the characteristics of platelet populations. We postulate that both the genetic make-up (inherited from megakaryocytes) and the vascular environment of a platelet determine its activation profile, and hence direct them to a specific population.

A population with unique properties is that of procoagulant platelets. ${ }^{14}$ These platelets are characterized by surface exposure of negatively charged phosphatidylserine (PS) at the outer leaflet of the plasma membrane, which property greatly enhances their interaction with vitamin K-dependent coagulation factors. ${ }^{15,16}$ In Chapter 5 we investigated how in particular the PS-exposing platelets are implicated in the extracellular shedding of glycoprotein (GP) receptors, mediated by the membrane-linked proteases ADAM10 and ADAM17. ${ }^{17,18}$ We discovered that shedding of both GPIba (by ADAM17) and GPVI (by ADAM10) is predominantly confined to the PS-exposing population (Figure 1), regardless of the type of stimulus, i.e. the $\mathrm{Ca}^{2+}$-elevating receptor ligands CRP-XL/thrombin or the apoptosis-inducing compound ABT-737. ${ }^{19}$ Kinetic experiments furthermore indicated that PS exposure can precede glycoprotein shedding. However, PS exposure (or platelet swelling) is not required for platelet ADAM activity, as shedding of GPIba in Scott platelets, which are devoid of PS exposure, was unaltered. The Scott platelets are deficient in both membrane phospholipid scrambling and swelling, because of a lack of the $\mathrm{Ca}^{2+}$-dependent ion channel anoctamin-6 (gene ANO6 or TMEM16F).20,21 Together, these findings indicate that ADAM activation in platelets occurs via regulatory mechanisms that are similar but not identical to those of PS exposure. Thus, glycoprotein shedding and PS exposure appear to be two independent events. This is in contrast to a recent report demonstrating a necessity for PS exposure to induce ADAM activity in lymphocytes. ${ }^{22}$

In Chapter 5, we also studied the signaling pathways leading to ADAM-mediated glycoprotein shedding. Inhibitor experiments indicated that both caspase activity (with ABT-737) and prolonged elevation in cytosolic $\mathrm{Ca}^{2+}$ (with CRP-XL/thrombin or $\mathrm{Ca}^{2+}$ ionophore) with a secondary role for protein kinase $\mathrm{C}$ activation could trigger shedding of GPIb $\alpha$ and GPVI. However, these pathways were not mutually exclusive, as the chelation of $\mathrm{Ca}^{2+}$ in ABT-737-stimulated platelets partly diminished GPIba shedding. This is in line with an earlier publication from our laboratory, showing the existence of a population of apoptotic platelets that shows increased levels of cytosolic $\mathrm{Ca}^{2+} .^{23}$

Another relevant finding was that cleavage of GPIb $\alpha$ and GPVI lowers the adhesion tendency of the platelets and, conversely, increases their procoagulant activity. It appeared that those platelets that had lost their GPIb $\alpha$ and GPVI receptors showed a decreased binding of VWF, but an increased binding of prothrombin and factor Xa. As a consequence, the platelets with cleaved receptors mediated a faster onset of 


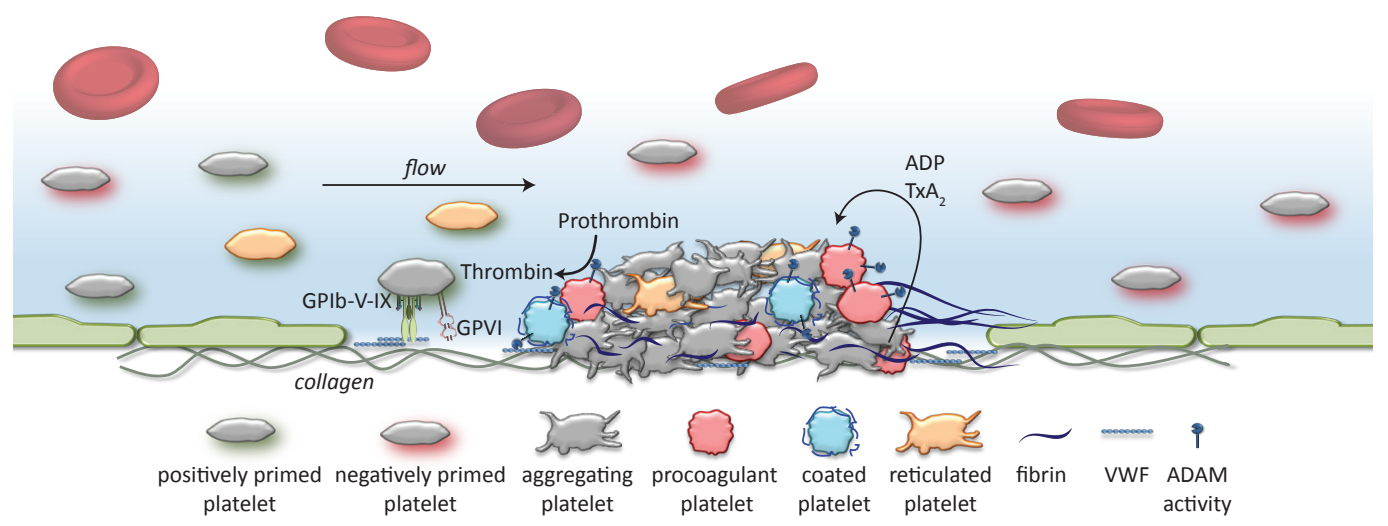

Figure 1. Proposed model of platelet priming and heterogeneity in thrombus formation. Platelets are positively or negatively primed by genetic and environmental factors and as such can show differential responses to vascular injury. This translates into the formation of a heterogenous thrombus with a distinct localization of the different platelet types and their specific responses (e.g. receptor shedding located specifically at the procoagulant platelets) within the thrombus. As such also the pattern of fibrin formation and thereby, the elasticity of the thrombus is affected.

fibrin formation under flow. Specific blockade of GPIba shedding also increased the procoagulant activity, suggesting a predominant role of GPIba in the control of this process.

At first sight a loss of glycoprotein receptors in procoagulant platelets is counterintuitive, because the literature reports that both GPIb $\alpha$ and GPVI can act as receptors for thrombin and fibrin, respectively, i.e. two major players of the coagulation cascade. ${ }^{24-26}$ Our data thus implicate that the platelet population that is most supportive of coagulation cannot steer this process via GPIba and GPVI. Accordingly, other populations must account for the GPIba- and GPVI-dependent effects of thrombin and fibrin, respectively: for instance, the populations of adhesive and aggregated platelets. In Chapter 4 we find that, at conditions where platelet adhesion is limited, thrombus formation is highly dependent on thrombin, whilst fibrin accumulates in the thrombus top. One might speculate that in such situations the relative importance of GPIba and GPVI as coagulant receptors increases. Glycoprotein-dependent binding of thrombin and fibrin may also facilitate the formation of procoagulant and coated platelet elsewhere in the thrombus.

\section{Acquired impairment of hemostasis: effects of reduced platelet count or dysfunction}

Given the central role of platelets as mediators of hemostasis, both an adequate platelet count (platelet mass) and platelet function is needed to prevent bleeding. ${ }^{27,28}$ In Chapter 2 we present the concept that platelets can be negatively or positively primed by their physiological environment, with positive priming becoming more important at conditions where the thrombosis risk increases. By changing the environment, not only 
anti-platelet but also anticoagulant therapies will affect the priming of platelets, and thereby alter their responsiveness. As a result, platelets can become hypo- or hyperresponsive (Figure 2 ).

Chapter 6 provides an illustration of how disease and treatment can affect platelet mass, function and priming. In this chapter, we investigated platelet and coagulant functions in patients, who had been diagnosed with a hematological malignancy and were treated with chemotherapy. As a consequence of disease and chemotherapy, these patients develop severe thrombocytopenia and are hence at increased risk for bleeding, ultimately with a necessity of platelet transfusion. ${ }^{29}$ Our data indicate that, after a couple of weeks of chemotherapy treatment, the remaining platelets of the patients are dysfunctional in multiple aspects. To a varying extent, these platelets display defective integrin activation, granule secretion and $\mathrm{Ca}^{2+}$ signaling. As the direct effect of chemotherapeutic exposure to the circulating platelets was minimal, it is concluded that the impairment in platelet function likely originates at the level of megakaryocytes. The high variability in platelet responses among patients could not be explained by the diagnosis or type of chemotherapy. This would argue for a variable susceptibility of the megakaryocytes. From patient to patient, the anti-proliferative effects of chemotherapeutics, e.g. inhibition of DNA replication and cell cycle progression, might differently affect the process of megakaryopoiesis. ${ }^{30}$ Furthermore, even megakaryocytic precursor cells have a different sensitivity to chemotherapeutics. ${ }^{31}$ This implies that the extent of damage inflicted to the megakaryocytes and derived platelets is determined by the chemotherapeutic agents administered and the stage of megakaryopoiesis.

Given the cytotoxic nature of chemotherapy, we investigated whether the dysfunction of the patient platelets was due to apoptosis. This was also driven by data that integrin activation and secretion are impaired in apoptotic platelets. ${ }^{32}$ However, we could not find indications of ongoing apoptosis in platelets from the patients. For instance, no caspase activity was detected, and caspase inhibition did not affect PS exposure. Rather, we found strong evidence that mitochondrial dysfunction could account for the diminished platelet responsiveness.

Under normal conditions, the energy demand increases upon platelet activation. As platelets rely on glycolysis and mitochondrial respiration for sufficient ATP production, an impairment in mitochondrial respiration can result in decreased platelet reactivity. ${ }^{33}$ Our data suggest that, in the patient platelets, the mitochondrial function is inadequate to support the higher energy demand needed for activation, with as a result lower platelet responsiveness. Mitochondrial dysfunction can also explain the increased PS exposure in the patient platelets, given the observation that mitochondria normally have a suppressive function in PS exposure. ${ }^{34}$ Along the same line, a deficiency in glucose metabolism can enhance PS exposure. ${ }^{35}$

Impaired platelet responsiveness appeared to be a main determinant of disturbed hemostasis in patients treated by chemotherapy (Chapter 6). On the other hand, the combined lowering of coagulation factors and platelets accounts for the increased bleeding risk in patients with dilutional coagulopathy during major surgery (Chapter 7). Here, we investigated the contribution of the plasmatic and cellular components of blood to the hemostatic potential, as measured by elastic fibrin clot formation (rotational thromboelastometry) and thrombin generation (assessed by the calibrated 
automated thrombin generation test). The method of rotational thromboelastometry or thromboelastography is frequently used to monitor the hemostatic capacity of the blood from patients during major surgery. ${ }^{36}$ Advantages of this point-of-care technique are that only small blood volumes are needed and that the measurement time is short. ${ }^{37,38}$ Our in vitro data suggest that upon dilutional coagulopathy - dilution of whole blood or plasma with crystalloids to $40 \%$ - fibrin clot formation could be normalized by reconstitution with either platelets or fibrinogen. On the other hand, administration of prothrombin complex concentrate (containing a mixture of pro- and anti-coagulation factors) or red blood cell concentrate was ineffective. In blood samples from patients taken before and after hemodilution, we found that only the platelet count and the fibrinogen level were relevant predictors of clot strength. Thrombin generation though could be restored by the addition of prothrombin complex concentrate, in which case addition of fibrinogen or platelets was without effect.

In Chapter 4, we used a whole blood perfusion assay to investigate thrombus and fibrin formation in blood obtained from patients with dilutional coagulopathy. Both thrombus and fibrin formation were severely impaired in these blood samples. In this test system - in contrast to rotational thromboelastometry - it appeared that reconstitution of both platelets and red blood cells was needed to achieve normal thrombus and fibrin formation at $40 \%$ dilution. The role of red blood cells herein, likely, is to improve platelet marginalization and adhesion to the microfluidic chamber surface..$^{39}$ Importantly, this platelet marginalization effect of (transfused) red blood cells, considered to be relevant for hemostasis (see Chapter 1), is not detected in other, static assays. Taken together, this work clearly indicates that the 'hemostatic benefit' of various blood products is often dependent on the type of test used. Accordingly, not every test outcome necessarily detects or reflects the actual bleeding risk of a patient.

\section{Normalization of hemostasis: recovery of platelet count and function}

Given that platelets, as anucleate cellular fragments, have only a low ability to synthesize proteins, ${ }^{40,41}$ the production of new platelets will be needed to repair any dysfunction (Figure 2). In Chapter 3, we investigated platelet function during one month after cessation of the irreversible $\mathrm{P}_{2} \mathrm{Y}_{12}$ antagonist prasugrel. We could demonstrate that the formation of juvenile platelets was needed for a recovery of $\mathrm{P}_{2} \mathrm{Y}_{12}$ signaling and platelet responses. However, surprisingly, this recovery was only partial during the first days after prasugrel discontinuation, and it only gradually increased during the offset period. These findings point to the presence of a 'reservoir' of inhibited $\mathrm{P} 2 \mathrm{Y}_{12}$ receptors in the megakaryocytes, which during the initial days of offset still produce $\mathrm{P} 2 \mathrm{Y}_{12}$-inhibited platelets.

This resembles the situation of chemotherapy-treated cancer patients, who also produce platelets with a megakaryocyte-inherited dysfunction (Chapter 6). Usually, the thrombocytopenia lasts for two to three weeks and then platelet count reverts to normal when chemotherapeutic treatments are stopped. ${ }^{29}$ Markedly, before the platelet count restores, the megakaryocyte ploidy increases, ${ }^{42}$ and the fraction of reticulated platelets rises. ${ }^{43,44}$ Our data indicate that an increase in platelet count - after discontinuation 
of chemotherapy - is accompanied by a normalization of function of the (reticulated) platelets. This suggests that the restoration process starts with a normalization of megakaryopoiesis.

In case of active or expected bleeding, the common treatment protocol in the Netherlands is a transfusion with pooled platelet concentrate. ${ }^{45,46}$ In Chapter 6, we found that platelet transfusion to patients receiving chemotherapy resulted in an improved platelet activity in thrombus and fibrin formation. These results are in agreement with other reports. ${ }^{47,48}$ Since the platelet concentrates used for transfusion purposes are stored for several days (median six days), this might be accompanied by a loss of mitochondrial function. ${ }^{49}$ However, no sign of this was found, since the mitochondrial membrane potential - a sensitive indicator for platelet quality ${ }^{50}$ - was comparable to that of freshly isolated platelets from healthy volunteers.

Inactivation in circulation Inactivation in megakaryocytes Formation of new active platelets Transfusion of blood components
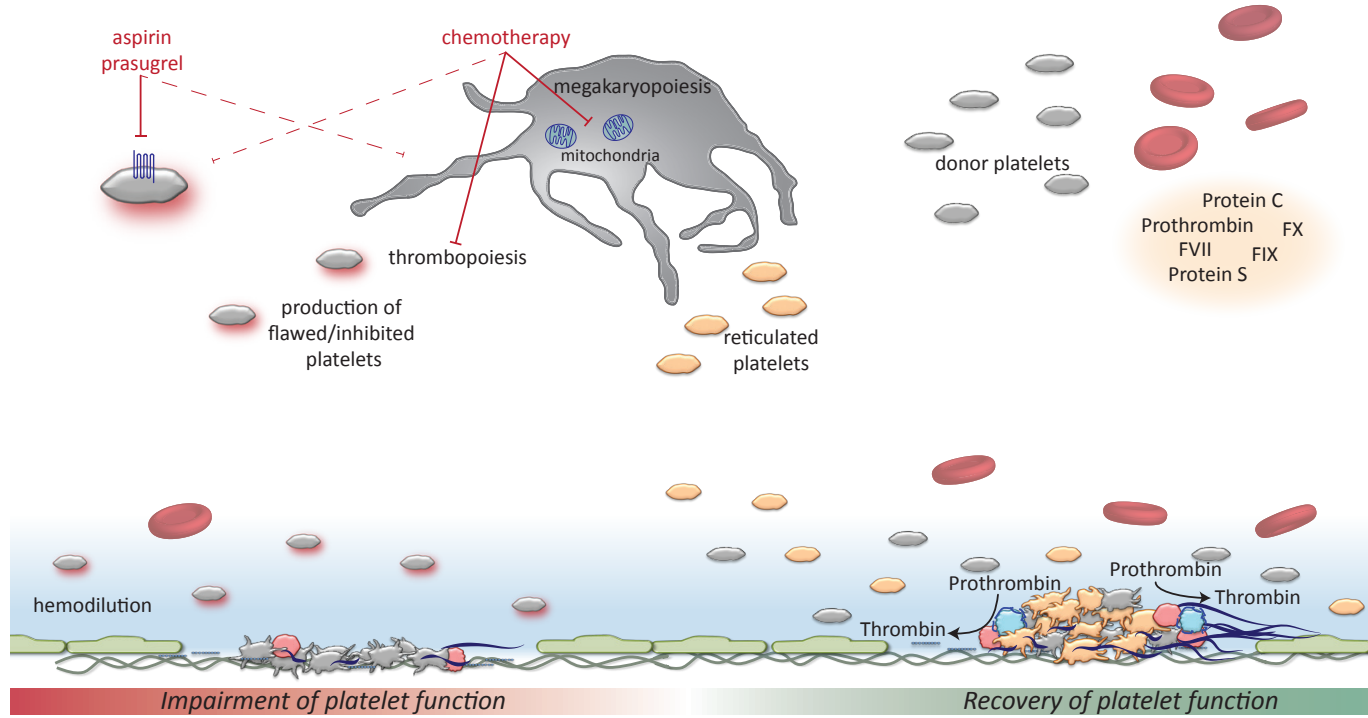

Figure 2. Mechanisms of impairment and recovery of platelet function. Impairment of platelet function can result from a direct inhibition of the platelets themselves during circulation and/or an indirect inhibition at the level of the megakaryocytes. Further, dilution of plasmatic and cellular components can affect platelet functionality as well. As platelets are anucleate cells, recovery of platelet function is dependent on the production of new platelets by megakaryocytes. When immediate improvement of hemostasis is required, transfusion of blood components can aid in the recovery of thrombus and fibrin formation. 


\section{Genetic alterations in hemostasis and thrombosis}

Chapter 8 describes a synthesis paper to compile the outcomes of published in vivo and in vitro studies on effects of gene depletion in mouse on arterial thrombus formation. This paper provides insight into the roles of 401 mouse genes in experimentally induced thrombosis with or without prolonged bleeding times. In particular, the synthesis approach enabled us to compare various methods employed to study in vivo and in vitro thrombus formation. Given that a conventional meta-analysis showed major limitations in numbers of studies to be included, we developed a three- and a five-point scoring scale in order to evaluate and combine results from individual published studies. The score normalization was used for a newly developed consistency analysis in order to determine the strength of the data from literature. This led to the identification of 19 genes, which regulated murine arterial thrombus formation, but were not implicated in tail bleeding. Remarkably, genes (proteins) that are currently targeted in the clinic by anti-platelet or anti-coagulant drugs are not included in this list, which is in agreement with increased incidence of bleeding seen with all these drugs. There is high interest in the development of new antithrombotic drugs that have a better antithrombotic efficacy without enhancing bleeding, e.g. by targeting receptors like GPVI (one of the 19 genes identified) or PAR1 (not expressed on mouse platelets). ${ }^{5,52}$

Another relevant outcome was that, in general, published results from in vivo thrombosis models (including thromboembolism) correlated well with those of in vitro flow chamber models, in particular for the collagen-dependent methods. This is of translational interest, since also with human blood, flow chamber tests are considered as a suitable initial screening tool for evaluating the efficacy of established and novel antithrombotic drugs. . $3,54^{-}$

Altogether, the findings of this thesis point to multiple interacting roles of the different blood components - platelets, red blood cells and plasma - in hemostatic reactions from platelet aggregation and thrombus formation to fibrin clot formation. Regarding platelets, it has become clear that different populations are formed with distinct properties and distinct interactions with other blood components. Although thrombocytopenia and platelet dysfunction often coincide, both conditions are relevant in a hemostatic imbalance. 


\section{References}

1. Munnix IC, Cosemans JM, Auger JM, Heemskerk JW. Platelet response heterogeneity in thrombus formation. Thromb Haemost. 2009;102(6):1149-1156.

2. Alberio L, Ravanat C, Hechler B, et al. Delayed-onset of procoagulant signalling revealed by kinetic analysis of COAT platelet formation. Thromb Haemost. 2017;117(6):1101-1114.

3. Podoplelova NA, Sveshnikova AN, Kotova YN, et al. Coagulation factors bound to procoagulant platelets concentrate in cap structures to promote clotting. Blood. 2016;128(13):1745-1755.

4. Lebois M, Josefsson EC. Regulation of platelet lifespan by apoptosis. Platelets. 2016;27(6):497-504.

5. Rinder HM, Tracey JB, Recht M, et al. Differences in platelet alpha-granule release between normals and immune thrombocytopenic patients and between young and old platelets. Thromb Haemost. 1998;80(3):457-462.

6. McBane RDn, Gonzalez C, Hodge DO, Wysokinski WE. Propensity for young reticulated platelet recruitment into arterial thrombi. J Thromb Thrombolysis. 2014;37(2):148-154.

7. Robinson MS, Mackie IJ, Khair K, et al. Flow cytometric analysis of reticulated platelets: evidence for a large proportion of non-specific labelling of dense granules by fluorescent dyes. Br J Haematol. 1998;100(2):351357.

8. Perl L, Lerman-Shivek $H$, Rechavia $E$, et al. Response to prasugrel and levels of circulating reticulated platelets in patients with ST-segment elevation myocardial infarction. J Am Coll Cardiol. 2014;63(6):513-517. 9. Stratz C, Bömicke T, Younas I, et al. Comparison of immature platelet count to established predictors of platelet reactivity during thienopyridine therapy. J Am Coll Cardiol. 2016;68(3):286-293.

10. Armstrong PC, Hoefer T, Knowles RB, et al. Newly formed reticulated platelets undermine pharmacokinetically short-lived antiplatelet therapies. Arterioscler Thromb Vasc Biol. 2017;37(5):949-956.

11. Stratz C, Nührenberg T, Amann M, et al. Impact of reticulated platelets on antiplatelet response to thienopyridines is independent of platelet turnover. Thromb Haemost. 2016;116(5):941-948.

12. Versteeg $\mathrm{HH}$, Heemskerk JW, Levi M, Reitsma PH. New fundamentals in hemostasis. Physiol Rev. 2013;93(1):327-358.

13. Swieringa F, Kuijpers MJ, Heemskerk JW, van der Meijden PE. Targeting platelet receptor function in thrombus formation: the risk of bleeding. Blood Rev. 2014;28(1):9-21.

14. Heemskerk JW, Mattheij NJ, Cosemans JM. Platelet-based coagulation: different populations, different functions. J Thromb Haemost. 2013;11(1):2-16.

15. Zwaal RF, Schroit AJ. Pathophysiologic implications of membrane phospholipid asymmetry in blood cells. Blood. 1997;89(4):1121-1132.

16. Bevers EM, Williamson PL. Getting to the outer leaflet: Physiology of phosphatidylserine exposure at the plasma membrane. Physiol Rev. 2016;96(2):605-645.

17. Gardiner EE, Karunakaran D, Shen Y, et al. Controlled shedding of platelet glycoprotein (GP)VI and GPIbIX-V by ADAM family metalloproteinases. J Thromb Haemost. 2007;5(7):1530-1537.

18. Andrews RK, Gardiner EE. Basic mechanisms of platelet receptor shedding. Platelets. 2017;28(4):319324.

19. Jackson SP, Schoenwaelder SM. Procoagulant platelets: are they necrotic? Blood. 2010;116(12):20112018.

20. Mattheij NJ, Braun A, van Kruchten R, et al. Survival protein anoctamin-6 controls multiple platelet responses including phospholipid scrambling, swelling, and protein cleavage. FASEB J. 2016;30(2):727-737.

21. Lhermusier T, Chap H, Payrastre B. Platelet membrane phospholipid asymmetry; from the characterization of a scramblase activity to the identification of an essential protein mutated in Scott syndrome. J Thromb Haemost. 2011;9(10):1883-1891.

22. Sommer A, Kordowski F, Büch J, et al. Phosphatidylserine exposure is required for ADAM17 sheddase function. Nat Commun. 2016;7:11523.

23. van Kruchten R, Mattheij NJ, Saunders C, et al. Both TMEM16F-dependent and TMEM16F-independent pathways contribute to phosphatidylserine exposure in platelet apoptosis and platelet activation. Blood. 2013;121(10):1850-1857.

24. Hantgan RR, Hindriks G, Taylor RG, Sixma JJ, de Groot PG. Glycoprotein Ib, von Willebrand factor, and glycoprotein IIb:Illa are all involved in platelet adhesion to fibrin in flowing whole blood. Blood. 1990;76(2):345-353. 
25. Mammadova-Bach E, Ollivier V, Loyau S, et al. Platelet glycoprotein VI binds to polymerized fibrin and promotes thrombin generation. Blood. 2015;126(5):683-691.

26. Alshehri OM, Hughes CE, Montague S, et al. Fibrin activates GPVI in human and mouse platelets. Blood. 2015;126(13):1601-1608.

27. Gieger C, Radhakrishnan A, Cvejic A, et al. New gene functions in megakaryopoiesis and platelet formation. Nature. 2011;480(7376):201-208.

28. Nugent D, Kunicki T. Platelet genomics: the role of platelet size and number in health and disease. Platelets. 2017;28(1):27-33.

29. Apelseth TO, Hervig T, Bruserud O. Current practice and future directions for optimization of platelet transfusions in patients with severe therapy-induced cytopenia. Blood Rev. 2011;25(3):113-122.

30. Vadhan-Raj S. Management of chemotherapy-induced thrombocytopenia: current status of thrombopoietic agents. Semin Hematol. 2009;46(Suppl 2):S26-S32.

31. Zeuner A, Signore $M$, Martinetti $D$, et al. Chemotherapy-induced thrombocytopenia derives from the selective death of megakaryocyte progenitors and can be rescued by stem cell factor. Cancer Res. 2007;67(10):4767-4773.

32. Vogler $M$, Hamali HA, Sun XM, et al. BCL2/BCL-X(L) inhibition induced apoptosis, disrupts cellular calcium homeostasis, and prevents platelet activation. Blood. 2011;117(26):7145-7154.

33. Kramer PA, Ravi S, Chacko B, Johnson MS, Darley-Usmar VM. A review of the mitochondrial and glycolytic metabolism in human platelets and leukocytes: implications for their use as bioenergetic biomarkers. Redox Biol. 2014;2:206-210.

34. Schoenwaelder SM, Darbousset R, Cranmer SL, et al. 14-3-3 $\zeta$ regulates the mitochondrial respiratory reserve linked to platelet phosphatidylserine exposure and procoagulant function. Nat Commun. 2016;7:12862.

35. Fidler TP, Campbell RA, Funari T, et al. Deletion of GLUT1 and GLUT3 reveals multiple roles for glucose metabolism in platelet and megakaryocyte function. Cell Rep. 2017;20(4):881-894.

36. Olde Engberink RH, Kuiper GJ, Wetzels RJ, et al. Rapid and correct prediction of thrombocytopenia and hypofibrinogenemia with rotational thromboelastometry in cardiac surgery. J Cardiothorac Vasc Anesth. 2014;28(2):210-216.

37. Schöchl H, Nienaber U, Hofer G, et al. Goal-directed coagulation management of major trauma patients using thromboelastometry (ROTEM)-guided administration of fibrinogen concentrate and prothrombin complex concentrate. Crit Care. 2010;14(2):R55.

38. Schols SE, Lancé MD, Feijge MA, et al. Impaired thrombin generation and fibrin clot formation in patients with dilutional coagulopathy during major surgery. Thromb Haemost. 2010;103(2):318-328.

39. Walton BL, Lehmann M, Skorcezewski T, et al. Elevated hematocrit enhances platelet accumulation following vascular injury. Blood. 2017;129(18):2537-2546.

40. Harrison P, Goodall AH. "Message in the platelet"-- more than just vestigial mRNA! Platelets. 2008;19(6):395-404.

41. Weyrich AS, Schwertz H, Kraiss LW, Zimmerman GA. Protein synthesis by platelets: historical and new perspectives. J Thromb Haemost. 2009; 7(2):241-246.

42. Bessman D. Prediction of platelet production during chemotherapy of acute leukemia. Am J Hematol. 1982;13(3):219-227.

43. Stohlawetz P, Stiegler G, Knöbl P, Höcker P, Panzer S. The rise of reticulated platelets after intensive chemotherapy for AML reduces the need for platelet transfusions. Ann Hematol. 1999;78(6):271-273.

44. Wang C, Smith BR, Ault KA, Rinder HM. Reticulated platelets predict platelet count recovery following chemotherapy. Transfusion. 2002;42(3):368-374.

45. Desborough MJ, Smethurst PA, Estcourt LJ, Stanworth SJ. Alternatives to allogeneic platelet transfusion. Br J Haematol. 2016;175(3):381-392.

46. Kreuger AL, Middelburg RA, Zwaginga JJ, van der Bom JG, Kerkhoffs JL. Clinical practice of platelet transfusions in haemato-oncology. Vox Sang. 2015;109(1):91-94.

47. Cauwenberghs S, Feijge MA, E. T, et al. Novel methodology for assessment of prophylactic platelet transfusion therapy by measuring increased thrombus formation and thrombin generation. $\mathrm{Br} \mathrm{J}$ Haematol. 2007;136(3):480-490.

48. Bikker A, Bouman E, Sebastian S, et al. Functional recovery of stored platelets after transfusion. Transfusion. 2016;56(5):1030-1037.

49. Perales Villaroel JP, Figueredo R, Guan Y, et al. Increased platelet storage time is associated with 
mitochondrial dysfunction and impaired platelet function. J Surg Res. 2013;184(1):422-429.

50. Verhoeven AJ, Verhaar R, Gouwerok EG, de Korte D. The mitochondrial membrane potential in human platelets: a sensitive parameter for platelet quality. Transfusion. 2005;45(1):82-89.

51. Weitz JI, Eikelboom JW, Samama MM. New antithrombotic drugs: Antithrombotic therapy and prevention of thrombosis, 9th edition: American college of chest physicians evidence-based clinical practice guidelines. Chest. 2012;141(2 Suppl):e120S-e151S.

52. Reimann A, Li Z, Goebel S, et al. Combined administration of the GPVI-Fc fusion protein Revacept with low-dose thrombolysis in the treatment of stroke. Heart Int. 2016;11(1):e10-e16.

53. Hosokawa K, Ohnishi T, Fukasawa M, et al. A microchip flow-chamber system for quantitative assessment of the platelet thrombus formation process. Microvasc Res. 2012;83(2):154-161.

54. Li R, Grosser T, Diamond SL. Microfluidic whole blood testing of platelet response to pharmacological agents. Platelets. 2017;28(5):457-462. 



\section{Chapter 10}

Summary

Samenvatting

Valorization

Curriculum Vitae

Publications

Dankwoord 

Summary 
Platelets play crucial roles in thrombosis and hemostasis. In an injured vessel wall they rapidly adhere, become activated and aggregate into a thrombus clot to halt bleeding. Similarly, following rupture of an atherosclerotic plaque, platelets activate, aggregate and form an intra-arterial thrombus. Alterations in platelet activation and/ or count can hence disturb the processes of thrombus and clot formation, with consequences for thrombosis or hemostasis. In this thesis, my emphasis is on acquired modes of platelet dysfunction and the underlying mechanisms of these.

Chapter 1 provides relevant background information on the regulation of platelet activation and thrombus formation. Highlighted are key platelet receptors responsible for adhesion to the vessel wall and, furthermore, the evidence for response heterogeneity between platelets. Also introduced are the clinical concepts how thrombosis is prevented by dual antiplatelet therapy, with bleeding as a side effect; as well as the conditions for platelet transfusion. In the review Chapter 2, a novel concept is presented how interplatelet heterogeneity can link to priming of platelets towards increased or decreased activation properties. Platelet heterogeneity can be observed at multiple levels. Structural heterogeneity can result from variability between individual megakaryocytes and from ageing of platelets in the circulation. Within a thrombus, response heterogeneity is enhanced by the specific location of the platelet in the thrombus and unequal distribution of receptor agonists, such collagen, ADP and thrombin. Regarding priming, during their time in circulation platelets will be exposed to a wide range of molecules that either increase or reduce the responsiveness. It is considered that, in pathological conditions, where the physiological balance between positive and negative priming factors is disturbed, platelets can become either hyper- or hypo-responsive. This can lead to an increased risk of thrombosis or bleeding, respectively. As a consequence of an underlying pathology, platelets can even be activated in the circulation, and subsequently appear as non-responsive, when assayed with in vitro tests. Therefore, we conclude that measurement is needed of both in vivo markers of platelet activation and in vitro activity of platelets, for a better understanding of the possible risks for thrombosis or bleeding.

In order to prevent recurrent thrombotic events, patients who suffered a myocardial infarction will receive dual antiplatelet therapy for one year, consisting of the cyclo-oxygenase inhibitor aspirin and an irreversible inhibitor of the ADP receptor $\mathrm{P} 2 \mathrm{Y}_{12}$, prasugrel. In Chapter 3 we investigated how the platelet activation properties recovered in patients, who stopped prasugrel intake after one year of treatment. Platelet responsiveness to ADP was hence tested at $0,1,2,5$ and 30 days after the cessation of drug intake. We found that after 5 days platelet responses to ADP were markedly improved, but still in part inhibited. In vitro addition of ticagrelor - a reversible P2Y antagonist - suppressed the regained ADP responsiveness after $>2$ days, thus providing evidence for a gradual recovery of $\mathrm{P} 2 \mathrm{Y}_{12}$-induced signaling. Interestingly, during the first days after discontinuation of prasugrel, we identified a separate population of platelets with fully regained responsiveness to ADP. Staining with the mRNA probes, thiazole orange or $5^{\prime}$-Cy-oligo-dT, showed that this population consisted of so-called juvenile platelets, i.e. newly released from the megakaryocytes residing in the bone marrow. Although the reactivity of the juvenile platelets was higher than that of older platelets, 
interestingly, the reactivity of both populations continued to increase during the whole offset period, suggesting a prolonged inhibitory effect of prasugrel intake on $\mathrm{P}_{2} \mathrm{Y}_{12}$ receptors of megakaryocytes.

In Chapter 4 we determined the interactions between immobilized collagen and tissue factor for platelet- and fibrin-dependent thrombus formation under flow conditions. It appeared that, alongside collagen that is required for platelet adhesion, the tissue factor-triggered generation of thrombin is a controlling factor determining the platelet-thrombus volume. In particular at low flow rates, the thrombin generated on procoagulant platelets drives the formation of fibrin fibres outside of the thrombus area. Furthermore, under conditions where platelet adhesion is a limiting factor, we find fibrin fibres redistributed from the bottom to the top of a thrombus. This chapter also elucidates the effects of the flow shear rate on the localization of fibrin and on the microelasticity of the fibrin-thrombus.

The glycoproteins (GP), GPIba and GPVI, are key surface receptors for platelet adhesion to von Willebrand factor and collagen, respectively. Both receptors are known to be susceptible to cleavage of their extracellular domains by the platelet membrane-bound extracellular proteases, ADAM10/17, in a process called receptor shedding. In Chapter 5 we investigated the signaling pathways underlying ADAM10/17 mediated receptor shedding as well as the implications of this process for platelet functions. Interestingly, we found heterogeneity in extent of shedding between populations of (activated) platelets. Platelets with phosphatidylserine exposure due to a high intracellular calcium level or a high caspase activity appeared to preferentially cleave the glycoproteins GPIb $\alpha$ and GPVI. Phosphatidylserine exposure also coincided with glycoprotein shedding in platelets of a thrombus. However, as the platelets from a Scott syndrome patient, which are unable to expose phosphatidylserine, still showed unchanged ADAM mediated cleavage of GPIba we concluded that phosphatidylserine exposure was not a prerequisite for shedding. Given the procoagulant effect of phosphatidylserine exposure, we subsequently investigated how receptor shedding affected the procoagulant potential of platelets. Remarkably, we found that this was increased by enhancing the binding of prothrombin to platelets and by accelerating fibrin formation.

In Chapter 6 we identified and characterized the dysfunction of platelets acquired by chemotherapy treatment in patients, who were diagnosed with a hematological malignancy and developed thrombocytopenia. We found that platelets from these patients show impaired integrin activation, granule secretion and spreading, when stimulated via their ADP, thrombin or collagen receptors. Markedly, the severity of the platelet dysfunction was not related to the disease type, the type of chemotherapy, or the platelet count. Moreover, platelet dysfunction was not explained by apoptosis, nor was the spontaneous phosphatidylserine exposure in the absence of agonists, since no caspase activity could be detected. Rather, mitochondrial impairments were associated with the compromised platelet responses as patient platelets showed a lower 
mitochondrial $\mathrm{O}_{2}$ consumption and a decreased mitochondrial membrane potential. Transfusion of the patients with platelet concentrates led to an overall recovery of responsiveness, thrombus formation and platelet-dependent fibrin formation.

As a consequence of substantial blood loss due to trauma or major surgery, infusion of crystalloids and/or colloid fluids is necessary for patients to maintain fluid and electrolyte homeostasis. However, the infusion also leads to dilution of the hemostatic factors and ultimately to an increased risk of bleeding, a condition known as dilutional coagulopathy. In Chapter 7 we investigated how addition of fibrinogen, platelets, red blood cells or prothrombin complex concentrate altered clot formation and thrombin generation in plasma samples resembling those of dilutional coagulopathy. We demonstrate that, in this case, fibrinogen as well as platelets can normalize the elastic clot formation assessed by thromboelastometry. In contrast, the impaired thrombin generation was particularly normalized by the addition of prothrombin complex concentrate. These results could be confirmed with blood obtained from patients before and after major fluid infusion. Here, again, it appeared that the platelet count and fibrinogen level were main predictors of the whole blood thromboelastometry outcome, in contrast to the hematocrit and prothrombin level. Clinical decisions on transfusion with fibrinogen or plasma, based on thromboelastometry measurements alone, should therefore take into account the platelet count.

In Chapter 8, a synthesis paper is presented of published in vivo and in vitro studies of murine arterial thrombosis and tail bleeding. In total 1407 studies were included, resulting in a comprehensive analysis of the roles of 401 mouse genes in thrombosis and hemostasis. By developing a scoring system of the pro- and antithrombotic effects of genetic modification, we found high correlations between gene effects of the most commonly used in vivo and in vitro models. Moreover, this synthesis identified 19 genes that solely affect arterial thrombosis without a role in bleeding. By constructing a network of human orthologues of murine genes that affect thrombosis and/or bleeding, we could identify multiple novel genes with a possible role in thrombosis and hemostasis. A role of several of these new genes was validated by studying thrombus formation in vitro using blood from eight genetically modified mouse models. This synthesis approach can provide a guide for the identification of new antithrombotic targets in the future.

In Chapter 9, the most important findings of this thesis are discussed in view of the current literature. It is reasoned that knowledge is needed of not only the platelet count, but also of the activation properties of the different platelet populations, in order to make an appropriate assessment of the hemostatic imbalance of patients with an (acquired) risk of thrombosis or bleeding. 


Samenvatting 
Bloedplaatjes spelen een cruciale rol bij zowel hemostase als trombose. Wanneer een bloedvatwand beschadigd raakt, leidt dat tot snelle aanhechting en activering van de bloedplaatjes. Het gevolg hiervan is een trombus van geaggregeerde plaatjes, die een bloeding kan stelpen. Ook na het scheuren van een arteriële atherosclerotische plaque worden plaatjes geactiveerd, en zij vormen dan een trombus die de arterie kan afsluiten. Als gevolg hiervan kunnen dus veranderingen in ofwel plaatjesactivering ofwel plaatjesaantal leiden tot een abnormale trombusvorming, en daarmee tot een verstoorde hemostase of verhoogde tromboseneiging. In dit proefschrift zijn dergelijke veranderingen onderzocht, met een nadruk op relevante verworven vormen van plaatjesdysfunctie en de mechanismen die daarvoor verantwoordelijk kunnen zijn.

Hoofdstuk 1 biedt relevante achtergrondinformatie over de regulatie van plaatjesactivering en trombusvorming. Uitgelicht zijn de voornaamste plaatjesreceptoren, zoals de receptoren die zorgen voor adhesie aan de vaatwand. Verder zijn de belangrijkste aanwijzigingen in kaart gebracht voor heterogeniteit tussen verschillende plaatjestypen. Om het klinische belang te benadrukken van een adequate onderdrukking van plaatjesactiviteit bij (arteriële) trombose beschrijft dit hoofdstuk ook de mogelijkheden tot preventie middels duale antiplaatjestherapie, die echter regelmatig bloedingen als bijwerking hebben. In Hoofdstuk 2 is een nieuw concept gepresenteerd over hoe de heterogeniteit tussen plaatjes van invloed kan zijn op het (on)gevoeliger maken (priming) van deze cellen voor stimulerende of inhiberende signalen. Deze heterogeniteit kan meerdere oorzaken hebben. Heterogeniteit in de samenstelling van plaatjes kan het gevolg zijn van verschillen tussen individuele megakaryocyten in het beenmerg, maar ook van een geleidelijke plaatjesveroudering. Binnen een trombus wordt de heterogeniteit tussen plaatjes ook bepaald door hun precieze locatie en daarmee samenhangend de ongelijke verdeling van plaatjesagonisten zoals collageen, ADP en trombine. Wat betreft het begrip priming worden plaatjes in de circulatie blootgesteld aan een scala van moleculen die hun responsiviteit stimuleren of juist remmen. Beargumenteerd is dat onder pathologische omstandigheden, waarin de fysiologische balans tussen positieve en negatieve priming-factoren verstoord is, de plaatjes van een patiënt hyper- danwel hypo-responsief kunnen worden. Dit kan leiden tot een verhoogd risico op trombose of bloeding. Als gevolg van een aandoening kunnen plaatjes in de circulatie zelfs geactiveerd worden en vervolgens dysfunctioneel raken, zoals dan blijkt uit in vitro testen. Op grond hiervan concluderen wij dat voor een goed begrip van de mogelijke risico's op trombose of bloedingen er metingen nodig zijn van zowel markers van de plaatjesactivering in vivo als de activiteit van geïsoleerde plaatjes in vitro.

Om een herhaalde trombose te voorkomen krijgen patiënten die een myocardinfarct hebben gehad een jaar lang duale antiplaatjestherapie, die bestaat uit de cyclo-oxygenase remmer aspirine en een irreversibele remmer van de $\mathrm{P}_{2} \mathrm{Y}_{12}$ receptor voor ADP, prasugrel. In Hoofdstuk 3 hebben we onderzocht hoe de plaatjesactivering herstelt in patiënten, die stoppen met prasugrel-behandeling. Daarvoor zijn de plaatjesresponsen op ADP gemeten op $0,1,2,5$ en 30 dagen na de laatste prasugrel-inname. Duidelijk werd dat op 5 dagen na stoppen de plaatjesrespons op ADP sterk verbeterd is, maar toch nog gedeeltelijk geremd. De herwonnen respons op ADP kon onderdrukt worden met ticagrelor - een reversibele $\mathrm{P}_{2} \mathrm{Y}_{12}$ antagonist. Dit bevestigde dus een gradueel herstel van de signalering via $\mathrm{P}_{2} \mathrm{Y}_{12}$ receptoren. Al op enige dagen na het stoppen van prasugrel-inname konden we 
een afzonderlijke populatie van plaatjes identificeren, waarvan hun responsiviteit op ADP grotendeels hersteld was. Door plaatjes te labelen met een mRNA marker zoals thiazoleoranje of 5'-Cy-oligo-dT, konden we aantonen dat de hoog-reactieve populatie bestond uit zogenaamde juveniele plaatjes, dat wil zeggen plaatjes die recent uit het beenmerg zijn vrijgekomen. Hoewel de reactiviteit van de juveniele plaatjes hoger was dan die van de oude (geremde) plaatjes, bleef de reactiviteit van beide populaties toenemen tijdens de periode na het stoppen van de inname van prasugrel. Dit suggereert dat het plaatjes-onderdrukkend effect van prasugrel verlengd wordt door remming van de $\mathrm{P}_{2} \mathrm{Y}_{12}$ receptoren van megakaryocyten in het beenmerg.

In Hoofdstuk 4 is nagegaan hoe een oppervlak met collageen met/zonder weefselfactor bepalend is voor plaatjesadhesie, trombusvorming en ontstaan van een fibrinestolsel onder stromingscondities. Uit het onderzoek bleek dat naast collageen dat de plaatjesadhesie initieert, de trombine die ontstaat middels weefselfactor bepalend is voor de grootte van de gevormde plaatjes-fibrinestolsels. Met name bij lage stromingscondities stimuleerde trombinegeneratie op het oppervlak van procoagulante plaatjes de vorming van fibrinevezels buiten de trombus. Daarnaast bleek dat onder omstandigheden, waarbij de plaatjesadhesie beperkt is, de fibrinevezels herverdelen van de onderkant naar de top van de trombus. Dit hoofdstuk beschrijft verder hoe de stromingssnelheid en de lokalisatie van fibrine bepalend zijn voor de microelasticiteit van een trombus.

Deglycoproteïnen (GP) GPIba en GPVI zijn belangrijke receptoren op het oppervlak van plaatjes voor hechting aan respectievelijk von Willebrand factor en collageen. Bekend is dat beide receptoren gevoelig zijn voor het knippen van hun extracellulaire domein door de extracellulaire proteasen ADAM10/17, welke zich ook op het plaatjesoppervlak bevinden. Dit knipproces wordt receptor shedding genoemd. In Hoofdstuk 5 hebben wij onderzocht welke signaleringspaden in plaatjes ten grondslag liggen aan deze ADAM10/17-gemediëerde receptor-shedding. Ook hebben we de gevolgen bepaald van het shedding-proces voor de functionaliteit van plaatjes. Een belangrijke bevinding was dat de shedding van zowel GPIb $\alpha$ als GPVI heterogeen verloopt, namelijk op bepaalde populaties van (geactiveerde) plaatjes. Vooral geactiveerde plaatjes met expositie van fosfatidylserine als gevolg van een hoge intracellulaire calciumconcentratie of een hoge caspase-activiteit bleken actief in het ADAM-gemedieerde knippen van GPIba en GPVI. Ook voor plaatjes in een trombus viel de glycoproteïne shedding samen met fosfatidylserine-expositie. Echter gezien de onveranderde shedding van GPIba in plaatjes van een patiënt met Scott syndroom, die dysfunctioneel zijn in fosfatidylserine-expositie, kon de conclusie getrokken worden dat fosfatidylserine-expositie geen vereiste is voor receptor-shedding. Gezien het procoagulante effect van fosfatidylserine-expositie, hebben we vervolgens onderzocht hoe shedding het stollingsvermogen van plaatjes beïnvloedt. Opmerkelijk vonden we een verhoogd stollingspotentiaal, omdat bij plaatjes met shedding de binding van stollingsfactoren toeneemt en de fibrinevorming versnelt.

Hoofdstuk 6 had als doel identificatie van de verworven dysfunctie van plaatjes in patiënten, die gediagnostiseerd zijn met een hematologische maligniteit, en na chemotherapie behandeling een trombocytopenie ontwikkelen. Aangetoond kon worden dat de plaatjes van deze patiënten een verminderde activering van integrines, een lage secretie van granula, en een defecte spreiding vertoonden, wanneer ze gestimuleerd 
werden met ADP, trombine of collageen. Typisch was dat de mate van plaatjesdysfunctie niet gerelateerd was aan de diagnose, het type chemotherapie of het plaatjesaantal. Bovendien kon noch de plaatjes-dysfunctie noch de spontane fosfatidylserineexpositie van plaatjes verklaard worden door actieve apoptose, aangezien geen activiteit van de apoptose-marker caspase gedetecteerd kon worden. Echter de lage plaatjesfunctie was geassocieerd met een verminderde activiteit van de mitochondriën. De patiënten-plaatjes vertoonden namelijk een verminderde zuurstofconsumptie en een verlaagd membraanpotentiaal in de mitochondriën. Transfusie van de patiënten met plaatjesconcentraat leidde tot een algeheel verbeterde plaatjesresponsiviteit, trombusvorming en plaatjes-afhankelijke fibrinevorming.

Toediening van kristalloïde en colloïde vloeistoffen is noodzakelijk om een goede vocht- en elektrolytenbalans te behouden, met name wanneer er bij een patiënt substantieel bloedverlies optreedt als gevolg van trauma of ingrijpende chirurgie. Echter deze vloeistof-infusie leidt ook tot een verdunning van hemostatische factoren in het bloed, en kan daarmee aanleiding geven tot een verhoogd bloedingsrisico. Deze conditie staat bekend als verdunningscoagulopathie. In Hoofdstuk 7 hebben wij onderzocht hoe in verdunde plasmamonsters de extra additie van fibrinogeen, bloedplaatjes, rode bloedcellen of protrombine complex concentraat (PCC) leidt tot een verbeterde trombinegeneratie en stolselvorming. Dit onderzoek werd uitgevoerd met plasmamonsters, die qua samenstelling vergelijkbaar waren met die van patiënten met verdunningscoagulopathie. In dit hoofdstuk konden we aantonen dat zowel fibrinogeen als plaatjesconcentraat een normaliserend effect heeft op de fibrinestolselvorming, zoals gemeten met tromboelastometrie. Anderzijds werd de lage trombinegeneratie het beste genormaliseerd door toevoeging van PCC. Deze conclusies werden bevestigd voor bloedmonsters verkregen van operatiepatiënten, bij wie het bloed verdund was door vloeistofinfusie tijdens de ingreep. Ook in deze metingen bleek dat het plaatjesaantal en de fibrinogeenconcentratie de belangrijkste voorspellende variabelen waren van de volbloed tromboelastometrie-test. Ook nu waren hematocriet en het protrombine-niveau veel minder relevant. In de klinische beslisregels omtrent transfusie van ofwel fibrinogeen ofwel plasma, die tegenwoordig vaak gebaseerd worden op tromboelastometriemetingen, zou daarom ook het plaatjesaantal opgenomen moeten worden.

In Hoofdstuk 8 is een vergelijkende kwantitatieve analyse beschreven van een groot aantal gepubliceerde studies naar arteriële trombose en staartbloeding in genetisch gemodificeerde muizen. In totaal zijn 1407 studies vergeleken, waarmee inzicht verkregen werd in de rol van 401 muizengenen bij arteriële trombose, trombo-embolisme en hemostase. Middels een scoringsprocedure voor de pro- en antitrombotische effecten van genetische modificatie konden we een hoge correlatie vaststellen van de uitkomst van de meest gebruikte in vivo en in vitro trombosemodellen. Sterkte-analyse resulteerde in 19 muizengenen die een positieve rol spelen bij arteriële trombose, maar geen effect hebben op bloeding. Na samenstellen van een netwerk van humane ortologe eiwitten van die muizengenen die trombose en/of hemostase beïnvloeden, konden we meerdere nieuwe eiwitten en genen identificeren die mogelijk betrokken zijn bij deze processen. 
Van een aantal van deze konden we een betrokkenheid valideren, door bestudering van de trombusvorming in vitro. Deze synthese-aanpak kan een toekomstige leidraad vormen voor de identificatie van nieuwe antitrombotische targets.

In Hoofdstuk 9 zijn de meest belangrijkste bevindingen van dit proefschrift bediscussieerd in het licht van de huidige literatuur. Verder is beredeneerd dat zowel het plaatjesaantal als de activeringseigenschappen van de verschillende plaatjespopulaties bekend dient te zijn om tot een beoordeling te komen van de hemostatische disbalans van patiënten met een (verworven) risico op trombose of bloedingen. 

Valorization 


\section{To valorize: to assign value or merit to}

Recent years more emphasis has been put on assigning social and/or economical value to scientific findings. ${ }^{1}$ However, the direct contribution to society is not as clear-cut for all scientific developments. To be able to improve the patient's quality of life or to ameliorate treatment, first a better understanding of the underlying physiological and pathological mechanisms has to be gained. Here, basic research is essential.

Platelets are an integral part of the hemostatic system. Upon vascular damage, platelets quickly respond by forming a platelet plug, which is stabilized by the formation of fibrin. Aberrant functionality of platelets can result in an imbalance in the hemostatic system and thereby contribute to thrombosis or hemorrhage. Arterial thrombosis underlies the development of cardiovascular disease, the number one cause of death globally, according to the World Health Organization. ${ }^{2}$ Here, antiplatelet drugs are an essential part in the treatment of cardiovascular disease. ${ }^{3}$ A downfall of antiplatelet therapy is the increased risk of hemorrhage. ${ }^{4}$ The high occurrence of bleeding complications as a result of antithrombotic therapy again underlines the delicate hemostatic balance, indicating the need for more insight into the processes of hemostasis and thrombosis to provide optimal treatment.

In this thesis two novel techniques are presented. In Chapter 3, we describe a new approach for the detection of juvenile platelets, i.e. the oligo-dT staining. The number of juvenile platelets is an indication of the platelet turnover, i.e. production of new platelets and degradation/consumption of circulating platelets. In different pathological conditions one or both processes can be affected. Knowledge of merely platelet count is insufficient to distinguish between a defect in platelet production or in degradation/ consumption. Here, the measurement of the number of juvenile platelets can be of great use. The current approach to detect juvenile platelets relies on the usage of thiazole orange to label mRNA, which is especially detectable in newly formed platelets. ${ }^{5}$ Since thiazole orange shows aspecific staining of the dense granules, results can be confounded by granular content. ${ }^{6}$ Although the procedures for oligo-dT staining are more laborious, the oligo-dT staining might be a suitable replacement for thiazole orange due to the higher specificity. The second novel technique is described in Chapter 4, in which a new integrative whole blood perfusion assay is presented that allows the simultaneous assessment of both platelet and coagulant functions under flow conditions. Most current assays only study either platelet function or coagulant activity under static conditions. However, platelets promote coagulation by providing a procoagulant surface for the assembly and activation of coagulation factors, which leads to enhanced generation of thrombin. Thrombin in turn is an important platelet activator. Using this novel whole blood perfusion assay, reciprocal effects of thrombus and fibrin formation (coagulation) can be studied, providing a better reflection of the patients' hemostatic potential.

In case immediate improvement of hemostasis is required, such as in conditions with a high risk of bleeding, transfusion of blood components is one of few treatment options available. ${ }^{7}$ However, transfusion of blood products entails several severe 
side effects such as allergic reactions, infections and incidentally acute lung injury. ${ }^{7}$ Furthermore, transfusion of blood products is associated with high costs. Currently, a substantial part of platelet concentrates $(67 \%)$ is administered to patients with severe thrombocytopenia as a consequence of hematological malignancies and chemotherapy treatment. ${ }^{8,9}$ For the prevention of bleeding these patients receive prophylactic platelet transfusions on guidance of the platelet count, i.e. when the platelet count drops below $10 \times 10^{9} /$ L. $^{8}$ However, in this patient population platelet count shows only a weak correlation with bleeding. ${ }^{10}$ In Chapter 6 we demonstrated that platelets from severely thrombocytopenic cancer patients treated with chemotherapy are highly dysfunctional in multiple aspects. We also showed variability in platelet responsiveness between patients, which was independent of platelet count. The next step would be to study whether platelet responsiveness is linked to the occurrence of bleeding. This may not only lead to better prevention of bleeding but also to a more efficient transfusion practice wherein only those patients will receive platelet concentrates that are likely to benefit. Ultimately this will reduce the number of platelet transfusions and thereby the negative side effects and high costs associated with it.

Patients, who suffer from severe blood loss due to trauma or surgery, may require fluid infusion to maintain electrolyte and fluid homeostasis. However, due to the large volumes of fluid infused, hemostatic factors are diluted as well, resulting in a condition better known as dilutional coagulopathy. In order to correct the dilution of the hemostatic factors, transfusion of blood products is necessary. In Chapter 4 and 7 , the contribution of multiple blood components to thrombus and fibrin formation was investigated. We demonstrated that the addition of fibrinogen and platelets ameliorated clot formation, while addition of prothrombin complex concentrate improved thrombin generation. The addition of red blood cells only proved to be beneficial for thrombus formation when thrombus formation was studied under flow using the whole blood perfusion assay. This highlights the importance of the incorporation of flow when studying thrombus and fibrin formation. Assays that are currently used in the clinic to monitor clot formation (e.g. thromboelastometry) measure platelet function and/or coagulant capacity under static conditions. Where thromboelastometry assesses hemostasis globally ${ }^{11}$ and thrombin generation determines the coagulant capacity in the absence or presence of platelets ${ }^{12}$, both assays lack the incorporation of shear stress. Here, whole blood perfusion assays such as described in Chapter 4, can be of great added value to determine the need and effect of transfusion in a clinical setting.

In order to gain more insight into thrombosis and hemostasis, in vivo studies are of great importance. Chapter 8 presents a synthesis of all published studies on thrombosis and hemostasis in genetically altered mice. By combining the results of all studies, several important observations could be confirmed and made. Firstly, in vitro models for thrombus formation correlated well to in vivo models for thrombosis. Thereby, in vitro whole blood perfusion models can partly replace and refine animal models. Secondly, by constructing a network of all genes, new genes that are highly likely to affect thrombosis and hemostasis, could be detected. Using this network, also new genes could be identified that affected thrombosis but not bleeding and are thereby, possible new targets for antithrombotic therapy. 


\section{References}

1. de Jonge B, Louwaars N. Valorizing science: whose values? Science \& society series on convergence research. EMBO Rep. 2009;10(6):535-9.

2. World Health Organization. Cardiovascular diseases (CVDs) 2017 [updated May 2017]. Available from: http://www.who.int/mediacentre/factsheets/fs317/en/.

3. Ten Berg JM, Zwart B, van 't Hof AW, Liem A, Waltenberger J, de Winter RJ, et al. Optimal duration of dual antiplatelet therapy after percutaneous coronary intervention or after acute coronary syndrome: Practical lessons from a review. Neth Heart J. 2017;25(12):655-63.

4. Cattaneo $\mathrm{M}$. The platelet $\mathrm{P} 2 \mathrm{Y}_{12}$ receptor for adenosine diphosphate: congenital and drug-induced defects. Blood. 2011;117(7):2102-12.

5. Harrison P, Goodall AH. "Message in the platelet"-- more than just vestigial mRNA! Platelets. 2008;19(6):395-404.

6. Robinson MS, Mackie IJ, Khair K, Liesner R, Goodall AH, Savidge GF, et al. Flow cytometric analysis of reticulated platelets: evidence for a large proportion of non-specific labelling of dense granules by fluorescent dyes. Br J Haematol. 1998;100(2):351-7.

7. Desborough MJ, Smethurst PA, Estcourt LJ, Stanworth SJ. Alternatives to allogeneic platelet transfusion. Br J Haematol. 2016;175(3):381-92.

8. Apelseth TO, Hervig T, Bruserud O. Current practice and future directions for optimization of platelet transfusions in patients with severe therapy-induced cytopenia. Blood Rev. 2011;25(3):113-22.

9. Estcourt LJ, Birchall J, Allard S, Bassey SJ, Hersey P, Kerr JP, et al. Guidelines for the use of platelet transfusions: a British society for haematology guideline 2016. Available from: http://b-s-h.org.uk/ media/2641/bcsh platelet guideline $0808 \quad 16 \mathrm{v} 2$. pdf.

10. Friedmann AM, Sengul H, Lehmann H, Schwartz C, Goodman S. Do basic laboratory tests or clinical observations predict bleeding in thrombocytopenic oncology patients? A reevaluation of prophylactic platelet transfusions. Transfus Med Rev. 2002;16(1):34-45.

11. Paniccia R, Priora R, Liotta AA, Abbate R. Platelet function tests: a comparative review. Vasc Health Risk Manag. 2015;11:133-48.

12. Al Dieri R, de Laat B, Hemker HC. Thrombin generation: what have we learned? Blood Rev. 2012;26(5):197203. 


Curriculum Vitae 
Constance Baaten was born on December $5^{\text {th }} 1988$ in Maasbracht, The Netherlands. In 2001 she started secondary school at the Trevianum Scholengroep in Sittard, where she received her gymnasium degree in 2007 . The same year she started the bachelor study of Molecular Life Sciences at Maastricht University. Here she received twice the Top 3\% Award of the Faculty of Health Medicine and Life Science. After finishing her bachelor degree, she obtained her Master's degree with the highest distinction (summa) cum laude at Maastricht University in Molecular Life Sciences - Oncology and Developmental Biology in 2012. Subsequently, she started as a PhD student at the Cardiovascular Research Institute Maastricht (CARIM) at the department of Biochemistry (Maastricht University, the Netherlands). Under supervision of Prof. Dr. J.W.M. Heemskerk and Dr. P.E.J. van der Meijden, she performed research in the field of thrombosis and hemostasis as described in this thesis. During this period, she worked in the laboratories of Prof. Dr. K. Kunzelmann (Regensburg, Germany), Prof. Dr. B. Nieswandt (Würzburg, Germany) and Prof. Dr. W. Ouwehand (Cambridge, United Kingdom). She presented her research at various international conferences in Bergamo (Italy), Leeds (United Kingdom) and Basel (Switzerland). Currently, she works as a researcher at the department of Biochemistry. 
Constance Baaten werd geboren op 5 december 1988 te Maasbracht. In 2001 begon ze haar middelbare schoolopleiding aan de Trevianum Scholengroep te Sittard. Aldaar behaalde ze haar Gymnasiumdiploma (profiel Natuur en Gezondheid) in 2007. Aan de Universiteit Maastricht startte ze met de bacheloropleiding Moleculaire Levenswetenschappen. In deze periode kon ze tweemaal de top 3\% onderscheiding van de Faculty of Health Medicine and Life Science in ontvangst nemen. Na het behalen van haar diploma in 2010, volgde ze aan dezelfde universiteit de masteropleiding Molecular Life Sciences met als specialisatie Oncology and Developmental Biology. In 2012 ontving ze haar masterdiploma met de hoogste onderscheiding, cum laude. Op 1 september 2012 begon ze als PhD-student bij het Cardiovascular Research Institute Maastricht (CARIM), binnen de Vakgroep Biochemie van de Universiteit Maastricht. Onder begeleiding van Prof. Dr. J.W.M. Heemskerk en Dr. P.E.J. van der Meijden voerde ze wetenschappelijk onderzoek uit in het domein van Trombose en Hemostase, zoals beschreven in dit proefschrift. Tijdens haar promotieperiode werkte ze in de laboratoria van Prof. Dr. K. Kunzelmann te Regensburg (Duitsland), Prof. Dr. B. Nieswandt te Würzburg (Duitsland) en Prof. Dr. W. Ouwehand in Cambridge (Verenigd Koninkrijk). Tevens presenteerde ze haar onderzoek op diverse internationale congressen in Bergamo (Italië), Leeds (Verenigd Koninkrijk) en Basel (Zwitserland). Momenteel is zij werkzaam als onderzoeker binnen de Vakgroep Biochemie. 

Publications 


\section{Full papers}

1. Ninivaggi M, Feijge MA*, Baaten $C^{*}$, Kuiper GJ, Marcus MA, ten Cate $H$, Lancé MD, Heemskerk JW, van der Meijden PE. Additive roles of platelets and fibrinogen in whole-blood fibrin clot formation upon dilution as assessed by thromboelastometry. Thromb Haemost. 2014; 111:447-57. *equal contribution

2. De Witt SM, Swieringa F, Cavill R, Lamers MM, van Kruchten R, Mastenbroek T, Baaten CC, Coort S, Pugh N, Schulz A, Scharrer I, Jurk K, Zieger B, Clemetson KJ, Farndale RW, Heemskerk JW, Cosemans JM. Identification of platelet function defects by multiparameter assessment of thrombus formation. Nat Commun. 2014; 5:4257.

3. Van Geffen JP, Kleinegris MC, Verdoold R, Baaten CC, Cosemans JM, Clemetson KJ, ten Cate $\mathrm{H}$, Roest M, de Laat B, Heemskerk JW. Normal platelet activation profile in patients with peripheral arterial disease on aspirin. Thromb Res. 2015; 135:513-20.

4. Baaten CC, Veenstra LF, Wetzels R, van Geffen JP, Swieringa F, de Witt SM, Henskens YM, Crijns H, Nylander S, van Giezen JJ, Heemskerk JW, van der Meijden PE. Gradual increase in thrombogenicity of juvenile platelets formed upon offset of prasugrel medication. Haematologica. 2015; 100(9):1131-8.

5. Mattheij NJ, Braun A, van Kruchten R, Castoldi E, Pircher J, Baaten CC, Wülling M, Kuijpers MJ, Köhler R, Poole AW, Schreiber R, Vortkamp A, Collins PW, Nieswandt B, Kunzelmann K, Cosemans JM, Heemskerk JW. Survival protein anoctamin- 6 controls multiple platelet responses including phospholipid scrambling, swelling, and protein cleavage. FASEB J. 2016;30: 727-37.

6. Mattheij NJ, Swieringa F, Mastenbroek TG, Berny-Lang MA, May F, Baaten CC, van der Meijden PE, Henskens YM, Beckers EA, Suylen DP, Nolte MW, Hackeng TM, McCarthy OJ, Heemskerk JW, Cosemans JM. Coated platelets function in platelet-dependent fibrin formation via integrin $\alpha \mathrm{llb} \beta 3$ and transglutaminase factor XIII. Haematologica. 2016: 101(4):427-36.

7. Swieringa F, Baaten CC*, Verdoold R*, Mastenbroek TG, Rijnveld N, van der Laan KO, Breel EJ, Collins PW, Lancé MD, Henskens YM, Heemskerk JW, van der Meijden PE. Platelet control of fibrin distribution and micro-elasticity in thrombus formation under flow. Arterioscler Thromb Vasc Biol. 2016; 36:692-9. *equal contribution

8. Baaten $\mathrm{CC}$, ten Cate $\mathrm{H}$, van der Meijden PE*, Heemskerk JW*. Platelet populations and priming in hematological diseases. Blood Rev. 2017; 31(6):389-99. *equal contribution

9. Baaten CC, Swieringa F, Misztal T, Mastenbroek TM, Feijge MA, Bock PE, Donners MM, Collins PW, Li R, van der Meijden PE, Heemskerk JW. Platelet heterogeneity in glycoprotein shedding: consequence for procoagulant responsiveness. Blood Advances, 2018: major revision.

10. Baaten CC, Moenen FC*, Henskens $\mathrm{YM}^{*}$, Swieringa $F$, Wetzels $R$, van Oerle $R$, Heijnen HF, ten Cate H, Holloway GP, Beckers EA, Heemskerk JW, van der Meijden PE. Impaired mitochondrial activity explains platelet dysfunction in thrombocytopenic cancer patients undergoing chemotherapy. Haematologica, 2018: major revision. *equal contribution 
11. Baaten CC*, Meacham S*, de Witt SM*, Feijge MA, Adams DJ, Akkerman JW, Cosemans JM, Jupe S, Kostadima M, Mattheij NJ, Prins MH, Ramirez-Solis R, Soehnlein O, Weber C, White JK, Ouwehand WH*, Heemskerk JW*. A synthesis approach of mouse studies to identify new genes and proteins in arterial thrombosis and bleeding. Submitted, 2018. *equal contribution

\section{Abstracts}

1. Ninivaggi $M$, Feijge MA, Kuiper GJ, Baaten CC, Marcus MA, ten Cate $H$, Lancé $M$, Heemskerk JW, van der Meijden PE. Principal roles of platelets and fibrinogen in whole-blood fibrin clot formation in dilutional coagulopathy determined by thromboelastometry. XXIV Congress of the International Society on Thrombosis and Haemostasis (ISTH), Amsterdam, The Netherlands; June-July 2013. J Thromb Haemost. 2013, 11: Suppl s2.

2. Mattheij NJ, Braun A, van Kruchten R, Cosemans JM, van der Meijden PE, Baaten CC, Ehlen H, Schreiber R, Vortkamp A, Collins PW, Bevers EM, Ousingsawat J, Kunzelmann K, Nieswandt B, Heemskerk JW. The Scott syndrome protein anoctamin 6 (TMEM16F) regulates multiple cell death responses including membrane phospholipid scrambling in platelets. XXIV Congress of the International Society on Thrombosis and Haemostasis (ISTH), Amsterdam, The Netherlands; June-July 2013. J Thromb Haemost. 2013, 11: Suppl s2.

3. Mattheij NJ, Braun A, van Kruchten R, Cosemans JM, van der Meijden PE, Baaten CC, Ehlen H, Schreiber R, Vortkamp A, Collins PW, Bevers EM, Ousingsawat J, Kunzelmann K, Nieswandt B, Heemskerk JW. The Scott syndrome protein anoctamin 6 (TMEM16F) regulates multiple cell death responses including membrane phospholipid scrambling in platelets. Proc. $15^{\text {th }}$ UK Platelet Group Meeting, Birmingham, United Kingdom; October 2012.

4. Baaten CC, Veenstra LF, Wetzels R, van Geffen JP, Swieringa F, de Witt SM, Henskens YM, Crijns H, Nylander S, van Giezen JJ, Heemskerk JW, van der Meijden PE. Ticagrelor to inhibit thrombogenicity of juvenile platelets. Proc. $1^{\text {st }}$ European Ticagrelor Science Conference, Barcelona, Spain, June 2014.

5. Baaten CC, Moenen FC, Henskens YM, Swieringa F, Wetzels R, van Oerle R, ten Cate $H$, Beckers EA, Heemskerk JW, van der Meijden PE. Multiple functional defects in platelets from thrombocytopenic cancer patients undergoing chemotherapy. ICTHIC, Bergamo, Italy, April 2016. Thromb Res. 2016, 140: Suppl 1:S171

6. Baaten CC, Swieringa F, Misztal T, Mastenbroek TG, Feijge MA, Donners MM, Collins PW, van der Meijden PE, Heemskerk JW. Heterogeneous regulation of platelet adhesive-receptor shedding in thrombus formation. Joint BSHT, AiP \& UK Platelet Group Meeting, Leeds, United Kingdom; November 2016.

7. Baaten CC, Swieringa F, Misztal T, Mastenbroek TG, Feijge MA, Donners MM, Collins PW, van der Meijden PE, Heemskerk JW. Heterogeneous regulation of platelet adhesive-receptor shedding in thrombus formation. GTH 2017, 61 ${ }^{\text {st }}$ Annual Meeting 
of the Society of Thrombosis and Haemostasis. Basel, Switzerland, February 2017.

8. Heemskerk JW, van Geffen JP, Baaten CC, Nagy M, de Witt SM, Brouns S, Tullemans $B$, Swieringa F, Kuijpers MJ, van der Meijden PE. In vitro thrombosis models. $10^{\text {th }}$ Congress on Alternatives and Animal Use in the Life Sciences, USA, Seattle, August 2017

9. Baaten CC, Moenen FC, Henskens $Y M$, Swieringa F, Wetzels R, van Oerle R, Heijnen $\mathrm{HF}$, ten Cate H, Holloway GP, Beckers EA, Heemskerk JW, van der Meijden PE. Impaired platelet responsiveness in thrombocytopenic cancer patients after chemotherapy as a consequence of mitochondrial dysfunction. XXVI Congress of the International Society on Thrombosis and Haemostasis (ISTH), Berlin, Germany; July 2017. RPTH. 2017, 1: Suppl s1.

10. Baaten CC, Swieringa F, Misztal T, Mastenbroek TM, Feijge MA, Bock PE, Donners MM, Collins PW, Li R, van der Meijden PE, Heemskerk JW. Mechanisms of platelet adhesive-receptor shedding in platelet populations in thrombus formation. XXVI Congress of the International Society on Thrombosis and Haemostasis (ISTH), Berlin, Germany; July 2017. RPTH. 2017, 1: Suppl s1.

11. Brouns SL, van Geffen JP, van Gorp RH, Baaten CC, van den Akker N, Schurgers LJ, van der Meijden PE, Heemskerk JW. Vessel wall components on a flow chip to understand the regulation of platelet thrombus formation and fibrin clot formation under flow conditions. 1 $^{\text {st }}$ Italian-UK platelet meeting, Bath, United Kingdom, September 2017.

\section{Oral and poster presentations}

1. Baaten CC, Moenen FC, Henskens YM, Swieringa F, Wetzels R, van Oerle R, ten Cate $\mathrm{H}$, Beckers EA, Heemskerk JW, van der Meijden PE. Multiple functional defects in platelets from thrombocytopenic cancer patients undergoing chemotherapy. ICTHIC, Bergamo, Italy, April 2016. Thromb Res. 2016, 140: Suppl 1:S171. (oral)

2. Kuijpers MJ, Baaten CC, Sabrkhany S, Moenen FC, Beckers EA, Henskens YM, Aarts MJ, Griffioen AW, oude Egbrink MG, Heemskerk JW, van der Meijden PE. Anti-cancer therapy inhibits platelet function. CARIM Symposium, Maastricht, The Netherlands, November 2016. (poster)

3. Baaten CC, Swieringa F, Misztal T, Mastenbroek TG, Feijge MA, Donners MM, Collins PW, van der Meijden PE, Heemskerk JW. Heterogeneous regulation of platelet adhesive-receptor shedding in thrombus formation. Joint BSHT, AiP \& UK Platelet Group Meeting, Leeds, United Kingdom, November 2016. (poster)

4. Baaten CC, Swieringa F, Misztal T, Mastenbroek TG, Feijge MA, Donners MM, Collins PW, van der Meijden PE, Heemskerk JW. Heterogeneous regulation of platelet adhesive-receptor shedding in thrombus formation. GTH Annual Meeting, Basel, Switzerland, February 2017. (oral)

5. Baaten CC, Moenen FC, Henskens YM, Swieringa F, Wetzels R, van Oerle R, Heijnen HF, ten Cate H, Holloway GP, Beckers EA, Heemskerk JW, van der Meijden PE. Impaired platelet responsiveness in thrombocytopenic cancer patients after chemotherapy 
as a consequence of mitochondrial dysfunction. XXVI Congress of the International Society on Thrombosis and Haemostasis (ISTH), Berlin, Germany; July 2017. RPTH. 2017, 1: Suppl s1. (poster)

6. Baaten CC, Swieringa F, Misztal T, Mastenbroek TM, Feijge MA, Bock PE, Donners MM, Collins PW, Li R, van der Meijden PE, Heemskerk JW. Mechanisms of platelet adhesive-receptor shedding in platelet populations in thrombus formation. XXVI Congress of the International Society on Thrombosis and Haemostasis (ISTH), Berlin, Germany; July 2017. RPTH. 2017, 1: Suppl s1. (poster)

\section{Awards}

Best oral presentation, GTH, Basel, Switzerland, 2017. 

Dankwoord 
Een boek zegt meer dan duizend woorden. (Herman Finkers)

Welgeteld bevat mijn boekje 68967 woorden. Deze woorden hadden echter nooit op papier gestaan zonder de hulp en steun van zovelen.

Allereerst, een groot dankjewel voor mijn promotieteam. Mijn promotor, Prof. Johan Heemskerk en mijn co-promotor, Dr. Paola van der Meijden. Dankjewel voor het vertrouwen en dat jullie mij de mogelijkheid hebben gegeven om mijn promotieonderzoek uit te voeren in jullie groep. Beste Johan, u maakt altijd tijd vrij voor vragen, hoe klein of groot ze ook zijn. Beste Paola, je zorgt altijd voor een translationeel aspect in de projecten en je laat me het belang van het onderzoek voor de patiënt niet vergeten, wat ik altijd zeer waardeer.

Leden van de leescommissie, Prof. Harry Schouten, Prof. Tilman Hackeng, Prof. Jaap Jan Zwaginga, Prof. Helen Philippou en Dr. Henri Spronk. Thank you for reviewing my thesis.

Mijn directe collega's, de superplatelet groep! Dankjewel voor alle gezelligheid, steun en hulp. Ik kom mede hierdoor elke dag met veel plezier naar het werk!

Allereerst mijn (oud)kamergenootjes. Magdi, working together with you is always great fun, wherever we are, Maastricht, Birmingham or Aachen. Thank you for all your help, support and friendship! Tom, je zorgde altijd voor veel gezelligheid op onze kamer, en een beetje extra tijdens de feestdagen. De kerstboom op de tafel naast me werd elk jaar groter! Ook dankjewel voor al je hulp bij het lay-outen van mijn boekje. Nadine en Susanne, jullie waren mijn allereerste kamergenootjes en zorgden er meteen voor dat ik me welkom voelde in de groep. Nadine, dankjewel dat je me wegwijs gemaakt hebt in de wereld van de facs-proeven. Ik zal ook nooit meer ons uitstapje naar het vliegveld van Luik vergeten. Susanne, met je aanstekelijke lach zorgde je altijd voor veel vrolijkheid op onze kamer en ik kan natuurlijk de goedgevulde snoepjespot niet vergeten. Daniëlle, jij bent er als laatste bij gekomen op onze kamer. Dankjewel voor alle gezelligheid.

Frauke, je zorgt altijd voor een beetje extra schwung op het lab, mede door de leuke muziek op de 'achtergrond'. Dankjewel voor al je hulp en positieve vibes bij de vele flowproeven. Marijke, Marion en Judith, ik kan bij jullie altijd terecht met al mijn vragen. Dankjewel voor alle goede raad en Marijke en Judith voor de gezelligheid op weg naar huis. Marion, dankjewel voor al je hulp bij de vele statistiek uitdagingen. Zonder jou was dat zeker niet gelukt!

Ook de aio-tjes van de andere kamer, Ankie, Bibian, Sanne en Mieke, dankjewel voor alle gezelligheid. Ankie, heel veel succes bij het afronden van jouw proefschrift, het wordt een heel mooi boekje! 
Siamack, dankjewel voor je hulp bij het 'vertalen' van de dokterscode in de patiënten informatie. Al die afkortingen waren abracadabra voor mij. Remco, dankjewel dat je mijn computer functionerend hebt gehouden! Simone, ik zal nooit de Sinterklaasavond vergeten bij mij thuis met jou als Sinterklaas en natuurlijk, de leuke verhalen en anekdotes die je altijd zo levendig vertelt. Stella, dankjewel voor al je hulp bij die moeilijke trombine generatie metingen.

Roger en Moniek, kort nadat ik was begonnen, gingen jullie weg uit de groep om aan een nieuwe uitdaging te beginnen. Dankjewel voor het doorgeven van jullie kennis.

Tomasz, it was a great pleasure to work with you. Thank you for all your help with those many facs experiments and for all those nice Polish sweets. I wish you all the best!

I would also like to thank all other employees at the Biochemistry department for the nice atmosphere and all the fun at our day-outs and events.

Yvonne, Floor, Erik, René, Rick, Paul, Daniëlle, Linda, Mariëlle, Carol, Inge, Paul, Lieve, Edmée en Lotte, dankjewel voor het includeren van de vele patiënten en het meten van de vele samples. Ook wil ik graag alle andere co-auteurs bedanken die hebben meegewerkt aan de projecten die in mijn thesis beschreven staan.

Dr. Noels, beste Heidi, dankjewel voor al je hulp bij het schrijven van de subsidieaanvragen. En nu hopen dat er eentje wordt toegekend!

Een speciaal bedankje voor alle vrijwilligers en patiënten die de afgelopen jaren bloed gedoneerd hebben. Zonder jullie bloeddonaties was mijn onderzoek niet mogelijk geweest.

Muziek maken brengt mij altijd veel ontspanning en plezier. Dankjewel aan iedereen van de muziekschool en het ensemble voor de mooie muziek.

Lisanne en Monique, dankjewel voor jullie vriendschap. Monique, na de middelbare school begonnen we samen aan ons MLW-avontuur en ook na onze studie, deelden we al ons promotie lief en leed. Ik wens je heel veel liefde en geluk toe samen met Mike in jullie nieuwe huis. Lisanne, al sinds groep 1 van de basisschool zijn we beste vriendinnen. We hebben samen heel wat leuke en soms minder leuke dingen meegemaakt. Dankjewel voor al je steun, je luisterend oor en natuurlijk alle gezelligheid! Heel veel succes met je opleiding en de laatste loodjes van jouw PhD avontuur. 
Tenslotte, mijn familie. Opa en oma, pap, Lenhart en mam, heel erg bedankt! Lenhart, al zou ik je soms het liefst achter het behang plakken, ik kan me geen betere broer wensen dan jij. Mam, ik kan alleen maar zeggen dankjewel voor echt alles.

Dankjewel allemaal!

\section{Constance}



